\title{
Colletotrichum: lifestyles, biology, morpho-species, species complexes and accepted species
}

\section{Jayawardena $\mathrm{RS}^{1,3}$, Bhunjun $\mathrm{CS}^{1,3}$, Hyde $\mathrm{KD}^{1,2,3,4^{*}}$, Gentekaki $\mathrm{E}^{1,3}$ and Itthayakorn $\mathbf{P}^{2}$}

\author{
${ }^{1}$ Center of Excellence in Fungal Research, Mae Fah Luang University, Chiang Rai, 57100, Thailand \\ ${ }^{2}$ Department of Biology, Faculty of Science, Chiang Mai University, Chiang Mai 50200, Thailand \\ ${ }^{3}$ School of Science, Mae Fah Luang University, Chiang Rai, 57100, Thailand \\ ${ }^{4}$ Innovative Institute of Plant Health, Zhongkai University of Agriculture and Engineering, Haizhu District, Guangzhou \\ 510225, People's Republic of China
}

Jayawardena RS, Bhunjun CS, Hyde KD, Gentekaki E, Itthayakorn P 2021 - Colletotrichum: lifestyles, biology, morpho-species, species complexes and accepted species. Mycosphere 12(1), 519-669, Doi 10.5943/mycosphere/12/1/7

\begin{abstract}
Colletotrichum is an important plant pathogenic genus that has undergone tremendous taxonomic changes. Species of Colletotrichum also occur as endophytes, saprobes and rarely entomopathogens. The current understanding of Colletotrichum taxonomy and application of various techniques in defining species within the genus is discussed in this paper. Here we provide a review of lifestyles, infection mechanisms, life cycle, host-specificity, classification history and techniques defining Colletotrichum species and the relation to speciation. Misidentifications and mistakes during species introduction are discussed and recommendations are provided for valid species publication. We provide an account of 248 currently accepted species with molecular data, which falls into 14 species-complexes and 13 singleton species. An updated account of Colletotrichum species is provided. Species are listed alphabetically in each species complex and annotated with their habitat, host, geographic distribution, phylogenetic position and typification details. Tables of host specific (152 species) and geographically endemic Colletotrichum species (19 species) are provided. A table of 450 morpho-species is provided for the first time gathering all data to one place, allowing mycologists to check before publishing a new species. Phylogenetic trees are provided for the whole genus and each species complex. Genes and combinations of genes that can be used for the identification of the species complexes are suggested. Future directions for the advancement of this genus are discussed.
\end{abstract}

Keywords - Host-specificity - Morpho-species - Pathogens - Singleton - Taxonomy

\section{Introduction}

Colletotrichum is the sole member of Glomerellaceae (Glomerellales, Sordariomycetes), and was introduced by Corda (1831) (Réblová et al. 2011, Maharachchikumbura et al. 2015, 2016, Hyde et al. 2020). Many species of this genus are important pathogens, while some are endophytes and saprobes (Cannon et al. 2012, Hyde et al. 2014, 2020, Jayawardena et al. 2016a, 2020). At the time of the first monographic treatment of Colletotrichum (von Arx 1957b), around 750 names existed (Cannon et al. 2012). von Arx (1957b) reduced this to 11 taxa based on morphological characters. Sutton (1980) accepted 22 species, while Sutton (1992) accepted 39 species based on 
morphological and cultural characteristics. Misidentification of Colletotrichum species occurs frequently due to only a few distinctive morphological characters being available for identification (Hyde et al. 2009a, Cannon et al. 2012, Jayawardena et al. 2016a). Hyde et al. (2009b) provided the first comprehensive overview of this genus with 66 names in common use and 19 doubtful names and also highlighted the need to revise this genus by using molecular methods (Hyde et al. 2009a). This study laid the foundation for the ongoing polyphasic research approaches on Colletotrichum (Cannon et al. 2012, Damm et al. 2012a, b, 2013, 2014, 2019, Weir et al. 2012, Liu et al. 2014, Jayawardena et al. 2016a, 2020).

Here we provide a review of lifestyles, infection mechanisms, life cycle, host-specificity, classification history and techniques for defining Colletotrichum species. We also discuss whether the scientific community has introduced too many species based on examples. This study uses Jayawardena et al. (2016a) as the starting point for accepted species, and also includes published records since that study. An overview of the currently accepted species in the genus along with their hosts, geographic distribution, phylogenetic position and sexual morphs are provided. Updated phylogenetic trees for the whole genus and the species complexes are also provided. When possible, the use of specific gene regions necessary to distinguish the species within the different species complexes is mentioned.

\section{Lifestyles}

Plant pathogenic fungi may have different lifestyles and infection mechanisms to colonise their hosts (Agrios 2005). Lifestyle patterns in Colletotrichum can be broadly categorised as endophytic, hemibiotrophic, necrotrophic, latent or quiescent (Perfect et al. 1999, Peres et al. 2005, Cannon et al. 2012, Barimani et al. 2013, de Silva et al. 2017). Hemibiotrophy is the most common among the above lifestyles (Cannon et al. 2012, Jayawardena et al. 2016a, de Silva et al. 2017). Being a complex genus, Colletotrichum has different lifestyles that vary between groups of species, many of which can switch between lifestyles (Promputtha et al. 2007, O'Connell et al. 2012). Most plant pathogenic taxa can change their relationships with the host at different stages of their life cycle. The physiological maturity and resistance of the hosts, environmental factors and virulence genes of the fungal taxa underpin the change (O'Connell et al. 2012, Stergiopoulos \& Gordon 2014). Species of Colletotrichum undergo major transformations in cell morphology and infection mode when switching lifestyles (O'Connell et al. 2012).

Specific gene families and biochemical interactions, such as the production of enzymes and secondary metabolites (Gan et al. 2016), regulate the lifestyle patterns of Colletotrichum species. Genomic- and transcriptomic-based research during the past decade has provided valuable insights into the relationship between genetics and lifestyles of Colletotrichum (Gan et al. 2013, 2016, 2019, Zeilinger et al. 2016). These studies have uncovered information on the evolution of lifestyles and their functional relationships and have also identified potential targets for control and management (Crouch et al. 2014). The available genomic data of Colletotrichum species comes from representatives of six independent species complexes (acutatum, destructivum, gloeosporioides, graminicola, orbiculare and spaethianum) and include endophytes, foliar and fruit pathogens (Liang et al. 2018). Comparative analyses of these genomes have revealed that Colletotrichum species may tailor their plant cell wall degrading enzymes and proteinases according to their lifestyle (O’Connell et al. 2012, Baroncelli et al. 2016, Hacquard et al. 2016). These genomes are also recorded to code for enzymes that catalyse the biosynthesis of secondary metabolites and are expressed in a phase-specific fashion (O'Connell et al. 2012).

Understanding different lifestyles of Colletotrichum and interactions with their hosts is important for plant biosafety and quarantine purposes (Bourget et al. 2013). However, changes in lifestyle patterns during the life cycle of a Colletotrichum species make detection and control difficult (de Silva et al. 2017). It is important to realize that export/import provide venues for species of Colletotrichum to disperse in asymptomatic plant materials over long distances and may result in the introduction of a completely new disease to a country. Therefore, understanding the lifestyles of this genus is important when considering quarantine measures and world trade 
(Newton et al. 2010, de Silva et al. 2017). In this section, we review the different lifestyles in Colletotrichum with examples.

\section{Biotrophic and hemibiotrophic lifestyles}

In a biotrophic lifestyle, the pathogen remains inside the living plant tissue and actively absorbs nutrients without killing the plant cells (Mendgen \& Hahn 2002). Different strategies to avoid defence responses of the host, such as masking of invading hyphae or active suppression of the defence, are essential for a biotrophic parasitic lifestyle (Münch et al. 2008). Without a biotrophic phase, the initial death of host cells could lead to recognition and initiation of host defence responses that could inhibit fungal growth (Münch et al. 2008). Even though Colletotrichum is not considered a true biotroph, many species do have a biotrophic stage in their life cycle, which is followed by a necrotrophic lifestyle, and is referred to as hemibiotrophic. This strategy may also contribute to the prominence of Colletotrichum species as symptomless endophytes in living plant tissues (Rojas et al. 2010, Cannon et al. 2012). Nascent colonies of many species of Colletotrichum enter a biotrophic phase, while infected tissues remain externally symptomless. This period may be short (1-3 days) or extended (probably dormant) (Prusky \& Plumbley 1992, Cannon et al. 2012). When Colletotrichum enters the necrotrophic stage, it results in the death of a significant number of plant cells, which is followed by the emergence of symptoms (Cannon et al. 2012, Jayawardena et al. 2016a).

Depending on host development stage and environmental conditions, the degree of hemibiotrophy varies among Colletotrichum species based on their lifestyle pattern and the timing of the switch from biotrophy to necrotrophy (Arroyo et al. 2005, Peres et al. 2005, Crouch et al. 2009b, da Silva et al. 2020).

\section{Endophytic lifestyle}

Endophytes are plant-inhabiting fungi that live within the host plant cells without causing any apparent disease symptoms (Rodriguez \& Redman 2000, Vieira et al. 2014). Depending on the physiological condition of the host plant, genotype and environmental conditions, the interaction between the plant and the endophyte can switch from mutualistic to antagonistic or pathogenic (Photita et al. 2004, Promputtha et al. 2007, Hardoim et al. 2015, da Silva et al. 2020). Phylogenetic analyses focusing on fungal interactions and lifestyles revealed that in most fungal lineages endophytic species were phylogenetically closer to species with typical necrotrophic lifestyles (Delaye et al. 2013). This suggested that the switch between endotrophy and necrotrophy has occurred multiple times in the evolutionary time scale. These findings support the idea that the breakdown of early mutualistic interactions lead to a pathogenic lifestyle (Stukenbrock \& McDonald 2008, Kiers et al. 2010, Eaton et al. 2011). Freeman \& Rodriguez (1993) genetically converted the phytopathogenic fungus $C$. magnum to a non-pathogenic endophytic fungus by knocking down a single gene. Abang et al. (2002) showed that an asymptomatic endophytic isolate of $C$. gloeosporioides was unable to produce a toxic metabolite, which is produced only by pathogenic strains and induces symptoms of disease in the host plant. Liu et al. (2015) identified six species from both symptomatic and asymptomatic leaf tissues, all of which belonged to gloeosporioides species complex namely C. camelliae, C. fructicola, C. gloeosporioides, $C$. jiangxiense, $C$. karstii and $C$. siamense providing convincing evidence that these species could switch their lifestyle from endophytic to pathogenic (Photita et al. 2001).

Many Colletotrichum species are symptomless endophytes in living plant tissues (Cannon et al. 2012, Jayawardena et al. 2016a, Ma et al. 2018). Infact Colletotrichum species are some of the most commonly identified endophytes (Tao et al. 2013, Jayawardena et al. 2016a, Marlin-Felix 2017, de Silva et al. 2021). Pathogenic Colletotrichum species have mutualistic interactions with their plant hosts providing disease resistance, drought tolerance and growth enhancements (Redman et al. 2001, Busby et al. 2016). The majority of Colletotrichum species within the boninense, gloeosporioides and graminicola species complexes are endophytes (Damm et al. 2012b, Weir et al. 2012, Vieira et al. 2014, Jayawardena et al. 2016a). Lu et al. (2004) identified endophytic strains of 
Colletotrichum from leaves of 12 forest tree species in Guyana, belonging mostly to the boninense and gloeosporioides complexes. Manamgoda et al. (2013) isolated and identified four endophytic species in the gloeosporioides complex. Ma et al. (2018) identified nine endophytic species from Dendrobium, of which two species belong to the gloeosporioides complex and four species in the boninense complex.

\section{Necrotrophic lifestyle}

Most of the species of Colletotrichum develop a necrotrophic stage in their life cycles (Cannon et al. 2012, O'Connell et al. 2012, de Silva et al. 2017). During this stage, the fungus actively infects and colonises the plant cells leading to death, and the appearance of disease symptoms (van Kan 2006, Cannon et al. 2012). Secondary hyphae start emerging from the primary hyphae invading the neighbouring cells (O'Connell et al. 2012). Necrotrophs typically secrete cell wall degrading enzymes, phytotoxins that kill the plant tissues and other extracellular enzymes into host tissues both before and during colonization (Laluk \& Mengiste 2010). Necrotrophic infection generally involves several stages: conidial attachment, germination, host penetration, primary lesion formation, lesion expansion, and tissue maceration, followed by sporulation (Prins et al. 2000). The pathogen subsequently survives on the contents of dead or dying cells and completes its life cycle (Laluk \& Mengiste 2010, Gan et al. 2013).

\section{Quiescent lifestyle}

Quiescence or latency is precluded as a major lifestyle, but as a transition phase towards the other phases of the life cycle (de Silva et al. 2017). Latency is defined as an extended period in the life cycle of a fungal pathogen, during which the fungus is dormant within the host before it switches to an active phase (Prusky et al. 2013). This lifestyle is important for species causing postharvest diseases, where the pathogen remains dormant inside the plant tissue before symptoms appear (Prusky 1996, Ranathunge et al. 2012). Physiological and biochemical changes during fruit ripening maintain or facilitate the transition from quiescent to necrotrophic lifestyle (Prusky et al. 2013). Colletotrichum gloeosporioides in avocado (Prusky 1996) and C. acutatum in almond (Adaskaveg \& Förster 2000) have a quiescent period in their lifestyle, before causing post-harvest disease. Ranathunge et al. (2012) reported a quiescent stage in $C$. truncatum after the initial infection of Capsicum annuum fruit.

\section{Infection mechanisms}

The infection mechanisms of fungi can provide insightful details to plant pathologists concerning the conditions required for a successful infection. Species of Colletotrichum can survive on a broad range of hosts and can develop unique relationships with them. Endophytic species have developed different abilities that aid them to be pathogenic or overwinter as saprobes. These asymptomatic infections may serve as inoculum sources for infection of other plants or the same plant (Freeman et al. 2001, Peres et al. 2005, Udayanga et al. 2013). The infection cycle of a Colletotrichum species can be reviewed mainly in two parts: pre-infection and post-infection.

\section{Pre-infection}

Penetration and colonisation with species of Colletotrichum generally starts with the germination of conidia and the formation of specialised infection structures called appressoria. These structures facilitate the entry of the fungus through the host cuticle and epidermal cell walls with the aid of a narrow penetration peg (Perfect et al. 1999, Latunde-Dada 2001, Wharton \& Schilder 2008, de Silva et al. 2017). There are rare cases recorded when the fungus infects through stromata or wounds, without forming appressoria, which is referred to as indirect penetration (Latunde-Dada et al. 1996, Latunde-Dada 2001). Species of Colletotrichum show forcible entry during direct penetration of host surfaces. Though conidial and appressorial adhesion are important in host penetration by forcible means; melanin and osmolytes are also required (Latunde-Dada 2001). Howard \& Ferrari (1989) mentioned that melanization is the result of selective permeability 
of the cell wall and in combination with the osmolytes in the appressorium the two generate enormous turgor pressure. Latunde-Dada (2001) noted that melanized appressoria of non-quiescent Colletotrichum species appear to be quite capable of non-enzymatic forcible penetration of intact host surfaces.

\section{Post-infection}

Colletotrichum species adopt either intracellular hemibiotrophy or subcuticular, intramural necrotrophy (Wharton \& Diéguez-Uribeondo 2004, Münch et al. 2008, O'Connell et al. 2012, de Silva et al. 2017).

\section{Intracellular hemibiotrophy}

This has an early stage of the symptomless biotrophic phase. During this phase, the penetration peg invades the epidermal cells and the primary hyphae produce enlarged infection vesicles inside epidermal and mesophyll cells. This is followed by the necrotic phase, in which secondary hyphae that secrete cell wall degrading enzymes, grow intracellularly killing the host cells (O'Connell et al. 2012). Latunde-Dada (2001) called this as 'stealth' and 'transient confinement' and summarized the avoidance mechanism of quiescent and endophytic Colletotrichum species through molecular and biochemical data showing that it is governed by many different co-related factors. Based on the behaviour of Colletotrichum species on cowpea and soybean, four assumptions were made by Latunde-Dada (2001). Formation of melanised appressoria readily occurs on leaf and stem surfaces by endophytic Colletotrichum species, followed by an extensive epiphytic colonisation. These melanised appressoria remain quiescent however, indirect penetration of young leaves through stromata occurs. With the subdivision of endophytic mycelium, asymptomatic extensive colonization of Sub-stomatal cavities and intercellular spaces of leaf mesophyll tissue, stem epidermis and cortex occur. During the final stage symptoms appear.

\section{Subcuticular, intramural necrotrophy}

In this phase, the fungus grows under the cuticle within the periclinal and anticlinal walls of the epidermal cells without penetrating the protoplasts (Moraes et al. 2013). There may also be a very short biotrophic phase before intramural hyphae aggressively initiate the destruction of the colonised host tissues (Curry et al. 2002, Arroyo et al. 2005, Peres et al. 2005, Ranatunge et al. 2012).

Most of the Colletotrichum species belong to different species complexes that are closely related to each other and have similar infection and colonization behaviour (Sanders \& Korsten 2003, Jayawardena et al. 2016a). However, the transition from one phase to another can vary depending on the host plant, host maturity and the particular species of Colletotrichum (de Silva et al. 2017). Species in the acutatum complex commonly start their life cycle with a short biotrophic phase and then switch to a necrotrophic phase (Freeman et al. 2001, Peres et al. 2005, Damm et al. 2012a). In the graminicola complex, species may have an extended biotrophic phase before switching to a necrotrophic phase (Münch et al. 2008, Freeman et al. 2001). In C. gloeosporioides, both intracellular hemibiotrophic and intramural necrotrophic infections were observed (Moraes et al. 2013). Colletotrichum truncatum was able to colonise chilli fruits through primary intramural hyphae fruit tissue without producing secondary biotrophic infection structures, before switching to the necrotrophic phase (Montri et al. 2009). Ranathunge et al. (2012) identified direct leaf cuticle penetration of $C$. truncatum followed by intramural, quiescent and necrotrophic phases of colonization. Species in the destructivum complex have a localized hemibiotrophic infection stage, in which the intracellular biotrophic phase is entirely confined within a single epidermal cell without extending into adjacent cells (Damm et al. 2014).

Understanding the infection mechanisms of this genus allows plant pathologists and plant breeders to develop better control strategies and plant varieties that are resistant against diseases. 


\section{Life cycle}

Understanding the life cycle of a fungus could lead to understanding of the fungus itself. Species of Colletotrichum have asexual and sexual morphs in their life cycle, which occur on the host plant or plant debris (Sutton 1992, Cannon et al. 2012, Jayawardena et al. 2016a). The asexual morph of Colletotrichum is well-studied compared to their Glomerella sexual morphs counterparts, as the former are most commonly associated with disease symptoms (Cannon et al. 2012, Jayawardena et al. 2016a). To complete the life cycle, the asexual morph will produce an acervulus, a structure formed intracuticularly in the plant tissue (Khan \& Hsiang 2003). The tension on the cuticle increases, once the fungal mycelium grows between the plant epidermis and cuticle. This leads to the breaking of the cuticle and exposure of the mature acervulus to the environment (Sutton 1992). Conidia are generated from the conidiophores arising from the stroma in the acervulus (Curry et al. 2002) and are wrapped in a water-soluble mucilaginous matrix. The mucilaginous matrix is composed of glycoprotein and germinating inhibitors that protect conidia against desiccation and toxins produced by the host defence mechanism (Leite \& Nicholson 1992). Upon contact with water (dew, irrigation and raindrops) and wind, conidia are disseminated to new locations, new hosts or the same host plant, and act as the primary inoculum for the new infection cycle (Ntahimpera et al. 1997). Sexual morph formation can be observed if the conditions are favourable (Sutton \& Shane 1983). The sexual morph of many Colletotrichum species is rarely observed in the natural environment, but readily occurs in cultures of species in boninense (C. annellatum, C. boninense, C. brassicola, C. constrictum, C. cymbidiicola, C. karstii, C. parsonsiae, C. petchii: Damm et al. 2012b, C. chiangraiense, C. citricola, C. doitungense: Ma et al. 2018), dracaenophilum (C. yunnanense: Damm et al. 2019, C. Parallelophorum: Ma et al. 2018), gloeosporioides (C. alienum, $C$. ciggaro, $C$. clidemiae, $C$. fructicola, $C$. salsolae, C. queenslandicum, C. ti, C. xanthorrhoeae: Weir et al. 2012), graminicola (C. graminicola: Vaillancourt \& Hanau 1991, 1992), magnum (C. cacao, C. magnum: Damm et al. 2019) and orchidearum (C. musicola, C. orchidearum, C. plurivorum, C. sojae, C. vittalense: Damm et al. 2019) complexes. Colletotrichum lentis and $C$. tanaceti in the destructivum complex produce sexual morphs by artificial crossing of isolates (Barimani et al. 2013). The life cycle of C. higginsianum when infecting Arabidopsis thaliana is given in Fig. 1.
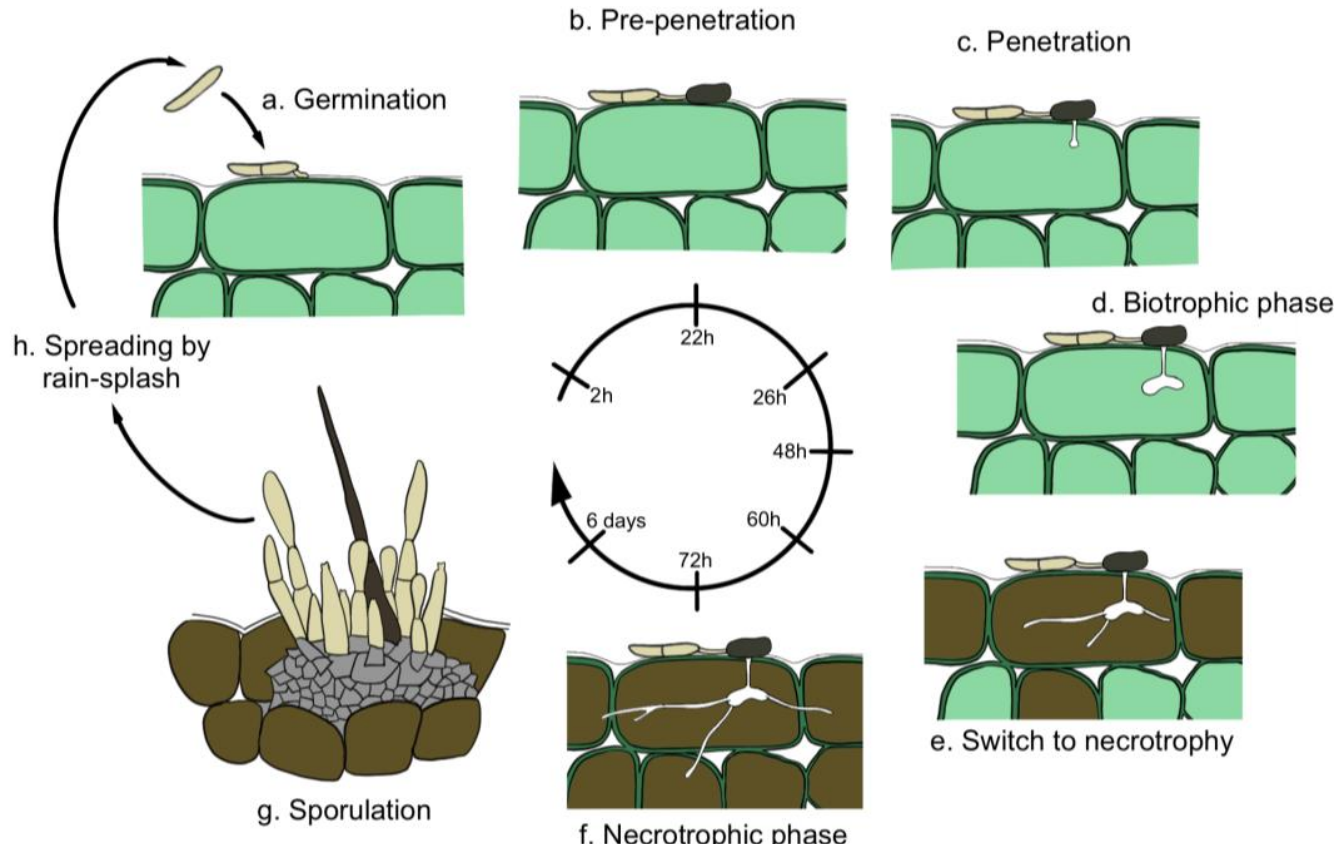

f. Necrotrophic phase

e. Switch to necrotrophy

Figure 1 - Life cycle of Colletotrichum higginsianum when infecting Arabidopsis thaliana (Redrawn from https://www.pilotunit.com (2020)). 
Genetic variation among species can be achieved through sexual reproduction. Perithecia can also act as survival structures, which help pathogens to overwinter or survive periods when a susceptible host is not available (Rodriguez-Guerra et al. 2005, Pinto et al. 2012). The size and shape of ascospores can be useful in the species-level diagnosis of Colletotrichum (de Silva et al. 2017). Sometimes the cultured isolates lose the ability to form perithecia due to storage conditions, nutrients in medium and frequent sub-culturing (Cannon et al. 2012, Jayawardena et al. 2016a). Therefore, reports mentioning species not producing their sexual morphs should be treated with caution.

Many studies have been conducted on Colletotrichum species as model organisms to understand their biological functions such as infection process, asexual-sexual relationships (Cannon et al. 2012). However, we conclude that more research is needed to understand the life cycle of the species of this genus.

\section{Are Colletotrichum species host-specific? Or does one species infect many hosts?}

In this section, we review host-specificity in Colletotrichum species. Previously, Colletotrichum species were assumed to be host-specific, leading to a large number of taxa being described from various hosts with little or no morphological variation (Hyde et al. 2009a, b, Cannon et al. 2012, Jayawardena et al. 2016a). Our understanding of host-specificity in Colletotrichum is rudimentary due to incomplete sampling, data mainly focusing on economically important crops and poor knowledge on the effect of pathogens (Cannon et al. 2012). The extent of the gene pool being sampled is significantly reduced, as most of the studies are restricted to understanding the species associated with a single crop (Gazis et al. 2011). Mackenzie et al. (2007) have demonstrated gene flow between populations of $C$. acutatum from native plants and those from adjacent strawberry crops. The infection strategy of Colletotrichum species is relevant to pathogenicity and host-specificity (see discussion above). Biotrophic fungi are usually hostspecific, while necrotrophs usually have broad host-specificity, but are vulnerable to non-specific host defence mechanisms (Vargas et al. 2012). Hemibiotrophic fungi combine both strategies: they have an initial biotrophic phase (which establishes host-specificity) followed by a virulent necrotrophic phase leading to the quick death of the target plants (Koek et al. 2011), making them especially destructive to their hosts. This makes hemibiotrophic fungi specifically qualified as biological control agents if they are host-specific (Goodwin 2001).

Some Colletotrichum species complexes contain taxa that exhibit some degree of hostspecificity (Weir et al. 2012, Jayawardena et al. 2016a). This may be due to limited sampling or to species concepts that assume specificity (Cannon et al. 2012). Damm et al. (2013) identified taxa in the orbiculare species complex that appeared to be restricted to specific herbaceous host species/genera (Asteraceae, Cucurbitaceae, Fabaceae and Malvaceae). Colletotrichum lindemuthianum appears to be restricted to Phaseolus vulgaris and P. coccineus in Fabaceae. Colletotrichum orbiculare causes diseases of several genera in Cucurbitaceae (Citrullus, Cucumis, Cucurbita, Lagenaria, Momordica and Trichosanthes) and some plant genera in Asteraceae (Xanthium sp.) and Malvaceae (Althaea sp.). Colletotrichum trifolii is recorded on Medicago and Trifolium (Fabaceae), while C. malvarum, C. tebeestii and C. sidae seem to be restricted to only one or few host species in Malvaceae. Colletotrichum bidentis and C. spinosum occur on Asteraceae. Taxa in the orbiculare species complex that establish hemibiotrophic relationships are distinctly host-specific. The reason for this is probably the close interactions that have developed during the initial, biotrophic phase of infection between the plant and the pathogen (Bailey et al. 1992).

Some species in the gloeosporioides complex also appear to be host-specific. Colletotrichum kahawae is recorded only from Coffea species in the African continent (Waller et al. 1993, Weir et al. 2012). Colletotrichum horii, C. nupharicola, C. ti and C. xanthorrhoeae also appear to be hostspecific. Colletotrichum ciggario, C. fructicola, C. siamense and C. theobromicola however are known to have a wider host range (Weir et al. 2012, Jayawardena et al. 2016a). Weir et al. (2012) pointed out that not all species with a wide host range are found everywhere. Colletotrichum 
alienum is commonly associated with cultivated fruit crops in New Zealand. However, C. fructicola and $C$. siamense, which are common on the same hosts in other parts of the world, are not found in New Zealand. Colletotrichum artocarpicola was isolated as a saprobe from Artocarpus heterophyllus in Thailand (Bhunjun et al. 2019). When the authors conducted cross pathogenicity studies, they found out that this species can be a pathogen not only of Artocarpus heterophyllus, but also of Carica papaya and Capsicum sp. (Shown in Figs 2a-2c).
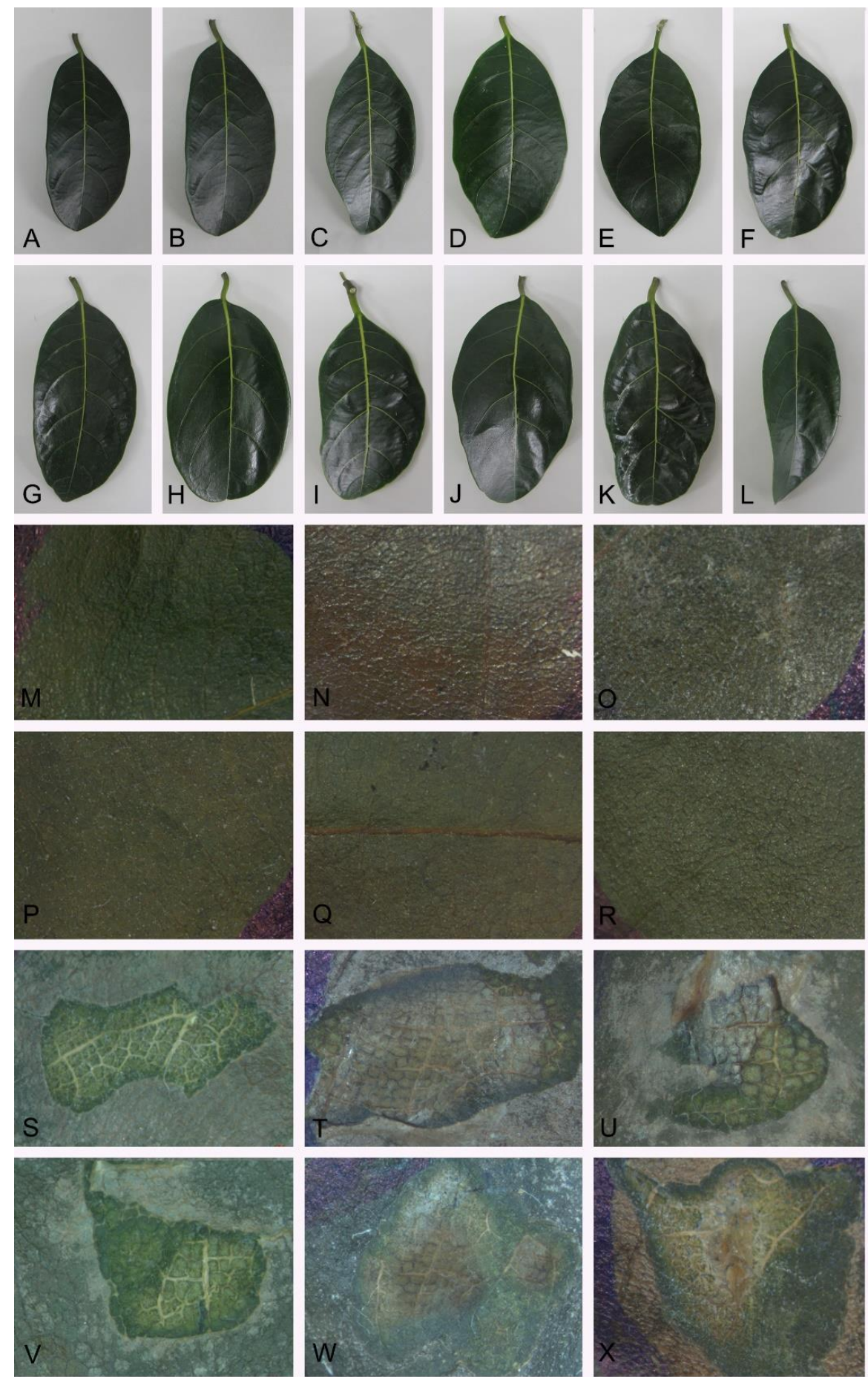

Figure 2a - Pathogenicity assay results of Colletotrichum artocarpicola on Jackfruit. A-L Jackfruit leaves selected for pathogenicity test. M, P Non-wounded negative control (conidial suspension and mycelium plug). N-O Non-wounded leaves inoculated with conidial suspension. Q-R Nonwounded leaves inoculated with colonised mycelium plug. S, V Wounded negative control (conidial suspension and mycelium plug). T-U Wounded leaves inoculated with conidial 
suspension showing sign of necrosis after 7 days. W-X Wounded leaves inoculated with colonised mycelium plug showing sign of necrosis after 7 days.
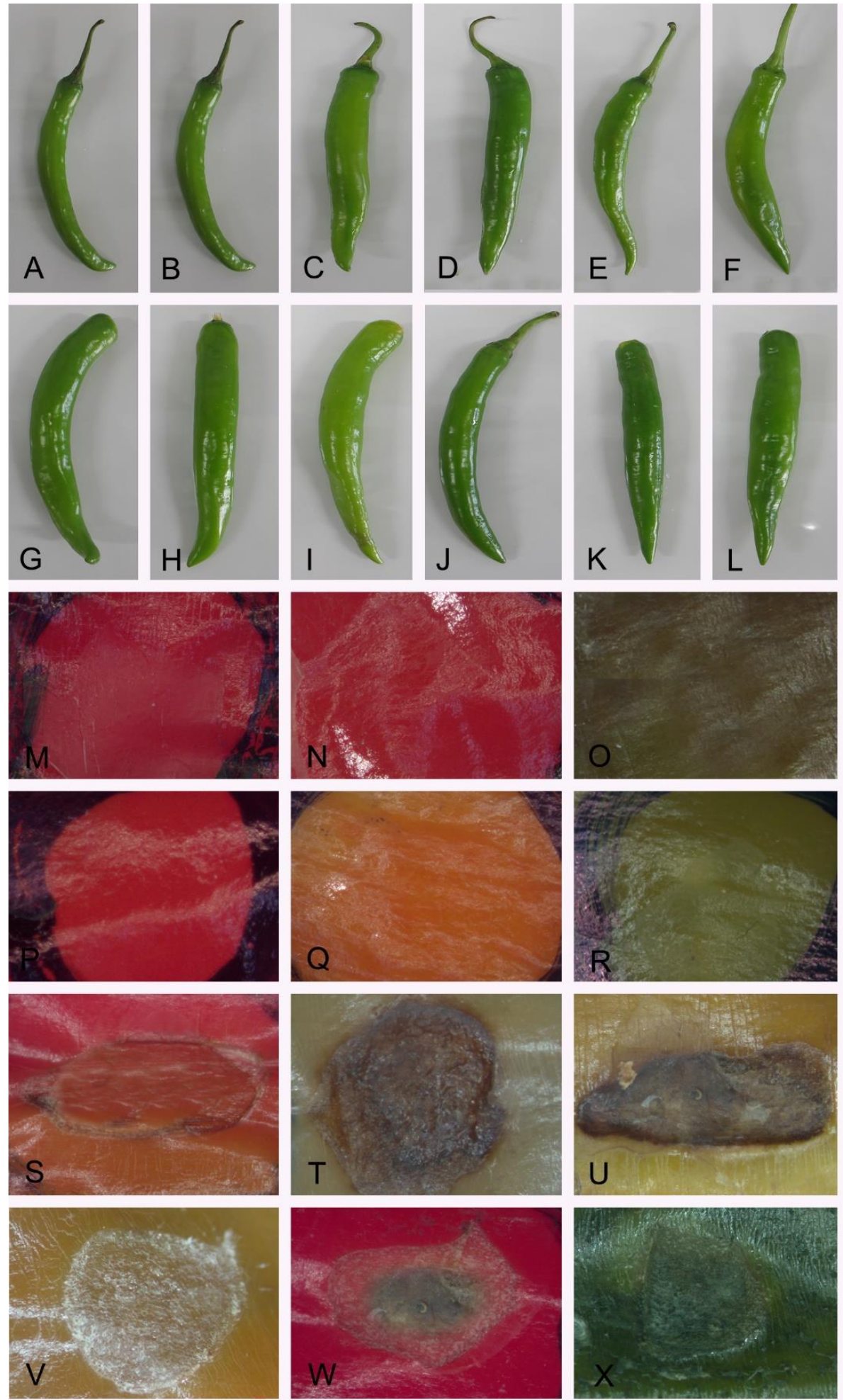

Figure 2b - Pathogenicity assay results of Colletotrichum artocarpicola on Chilli. A-L Chillies selected for pathogenicity test. M, P Non-wounded negative control (conidial suspension and mycelium plug). N-O Non-wounded chillies inoculated with conidial suspension. Q-R Nonwounded chillies inoculated with colonised mycelium plug. S, V Wounded negative control (conidial suspension and mycelium plug). T-U Wounded chillies inoculated with conidial suspension showing sign of necrosis after 7 days. W-X Wounded chillies inoculated with colonised mycelium plug showing sign of necrosis after 7 days. 


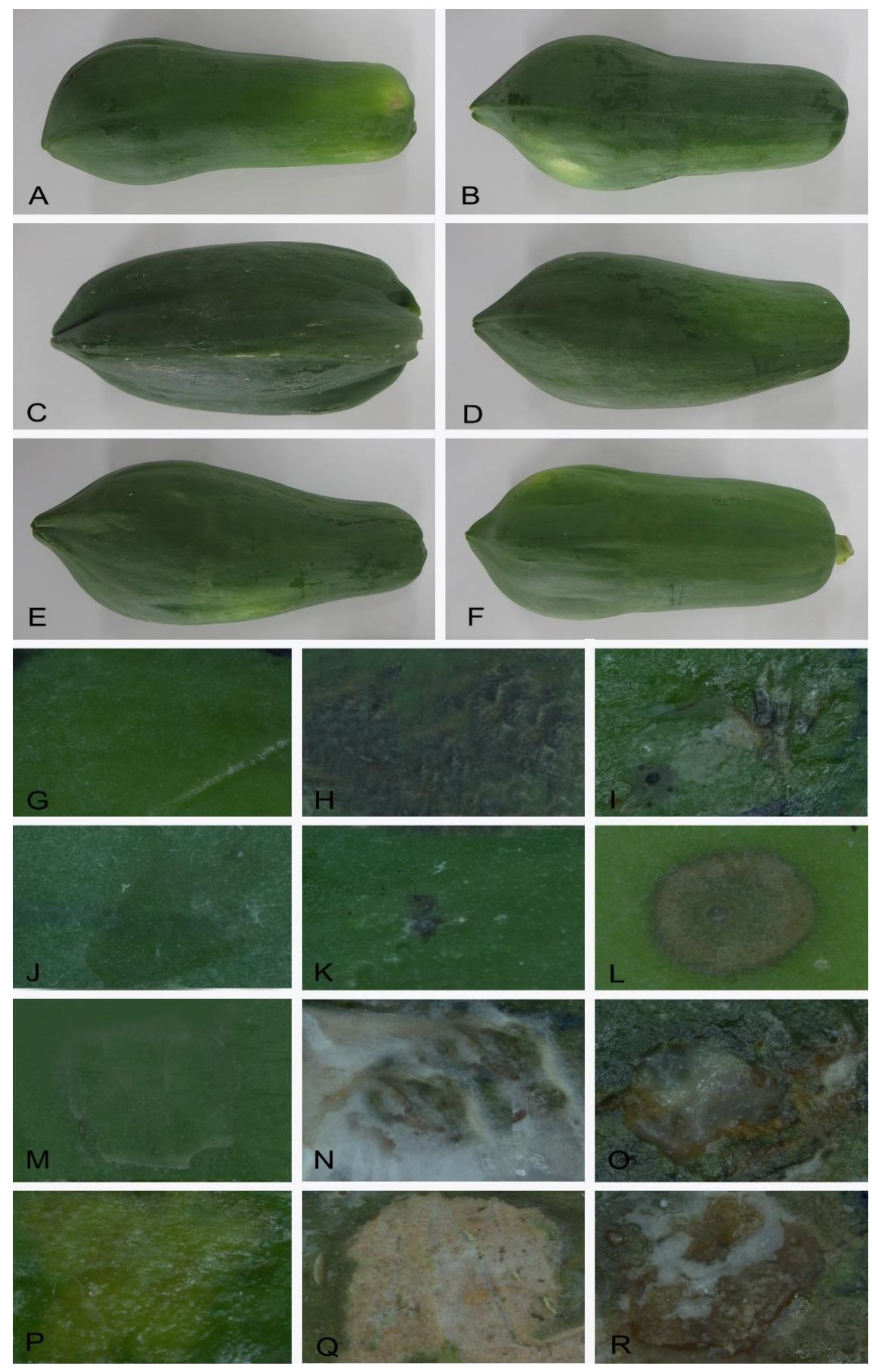

Figure 2c - Pathogenicity assay result of Colletotrichum artocarpicola on Papaya. A-F Papayas selected for pathogenicity test. G, J Non-wounded negative control (conidial suspension and mycelium plug). H-I Non-wounded papayas inoculated with conidial suspension. K-L Nonwounded papayas inoculated with colonised mycelium plug. M, P Wounded negative control (conidial suspension and mycelium plug). N-O Wounded papayas inoculated with conidial suspension showing sign of necrosis after 14 days. Q-R Wounded papayas inoculated with colonised mycelium plug showing sign of necrosis after 14 days.

Species of the caudatum and graminicola complexes appear to be restricted to single host species or genera in Poaceae (Crouch et al. 2009, Crouch 2014). Colletotrichum truncatum, an intramural pathogen grows only in cell walls and has wide host range (Roberts \& Snow 1990, Jayawardena et al. 2016a). In the destructivum species complex, some species appear to be specific to certain genera or families of herbaceous plants (Damm et al. 2014). Colletotrichum fuscum is recorded only on Digitalis, while C. higginsianum is recorded on genera of Brassicaceae. In 
contrast, other species can be considered as generalists occurring on taxonomically diverse plant families. Damm et al. (2014) revealed that C. destructivum occurs on Asteraceae, Fabaceae and Polygonaceae, while C. lini occurs on Asteraceae, Brassicaceae, Fabaceae, Lamiaceae, Linaceae and Ranunculaceae. In their study, they also highlighted several host species that can be infected by more than one taxon of the destructivum complex. For example, Medicago and Trifolium are infected by three species in the destructivum complex, while Raphanus and Pisum are infected by two species.

Following in-vitro infection studies, Whitelaw-Weckert et al. (2007) revealed the low specificity among isolates assigned to $C$. acutatum, C. simmondsii and C. fioriniae. Damm et al. (2012a) mentioned that the lack of perceived host-specificity in the $C$. acutatum complex rests with poor identification practices in pathology studies. Colletotrichum acutatum has been recorded as a pathogen of countless host plants with a wide geographical distribution (Farr \& Rossman 2021). However, Sreenivasaprasad \& Talhinhas (2005) found that C. acutatum occurs only on certain hosts, mostly in the southern hemisphere. Damm et al. (2012a) confirmed that species of the acutatum complex do occur on multiple hosts, but the known host spectrum is much smaller than previously thought. Species of the acutatum complex have rarely been found in Europe, and mostly on ornamental plants (Damm et al. 2012a). Hosts that are infected by more than one species of the acutatum species complex include apple, citrus, olive, cranberry, blueberry and strawberry. For example, five species of Colletotrichum are associated with Malus (C. acerbum, C. fioriniae, C. godetiae, $C$. nymphaeae and $C$. salicis). Talhinhas et al. (2005) found five groups, (now recognised as species within the acutatum species complex) occurring on olives in Portugal (C. acutatum, $C$. fioriniae, $C$. godetiae, $C$. nymphaeae and $C$. rhombiforme).

Within the boninense species complex, some species are host-specific, or at least show a preference for hosts from particular plant groups (Damm et al. 2012b). Based on ITS1 sequence data, Morikawi et al. (2003) detected four molecular subgroups within $C$. boninense. In their analyses, strains from monocotyledonous and dicotyledonous plant hosts were separated. The monocotyledonous clade consisted of three subclades. Of these subclades, one is restricted to Orchidaceae and the other two contain strains from Amaryllidaceae. However, the dicotyledonous clade did not show any clear host-linked substructure. Colletotrichum karstii while occurring on a wide range of dicotyledons was also described on Orchidaceae and also occurs on other monocotyledons (Damm et al. 2012b). Damm et al. (2012b) recognised C. dracaenae and $C$. hippeastri to be specific at the host genus level. However, the number of strains that were examined was insufficient to confirm this.

Damm et al. (2019) mentioned that many species in the dracaenophilum, magnum and orchidearum complexes are possibly host-specific. Some species occur only on monocotyledons, while others occur across a broad spectrum of plant hosts. Most species in these complexes have predominantly been isolated from plants in tropical or subtropical regions (mainly from Asia and Latin America), while there are a few collections from ornamental plants in temperate climates (in Central-Europe: Damm et al. 2019). A few host-specific species are found in the dracaenophilum complex. However, this can be a result of insufficient sampling. In the orchidearum species complex $C$. orchidearum, $C$. plurivorum and $C$. sojae are very common and occur in many host species. Colletotrichum cliviicola, C. musicola, $C$. cattleyicola and $C$. piperis are less common species that seem to be either host-specific or restricted to a specific country and region (C. vittalense: Damm et al. 2019). With the exception of $C$. brevisporum and an undescribed taxon, species in the magnum complex are recorded only from a single host. However, this can be a result of insufficient sampling as these species have been introduced based only on a single strain.

The interpretation of host-specificity in Colletotrichum species may be more appropriately addressed using the information on their ability to cause disease rather than occurrence. This is important in the case of endophytic strains, where the identity of the host may be incidental (Lu et al. 2004). The ability to infect and the degree of virulence are determined by rapidly evolving secreted effector proteins that facilitate infection, thus suppressing plant immunity (Schulze-Lefert \& Panstruga 2011). Comparative genomic analyses of the effector repertoires of "specialist" and 
"generalist" members of Colletotrichum could accordingly provide important insights into the molecular basis of their ability of infection and degree of and virulence within the genus. For example, O'Connell et al. (2012) uncovered major differences in the gene complement of $C$. higginsianum and $C$. graminicola using comparative genomics, despite their similar morphologies. They proposed that the diversification of functions required for host interaction, particularly, the secretion of small-molecule and protein effectors and the degradation of plant polymers, allows $C$. higginsianum to colonize a wider range of plant species (generalist). In contrast, $C$. graminicola, a pathogen that is adapted to a narrow range of hosts, has maintained a more targeted arsenal of virulence factors (specialist). Gene family contractions are evident in Colletotrichum and are associated with narrow host ranges (Baroncelli et al. 2016), suggesting that host-specificity may be linked to gene function loss (Stajich 2017). Gan et al. (2016) sequenced the genome of a strain of $C$. incanum (in the spaethianum complex) and showed that it can infect both monocotyledons and dicotyledons, providing a new and unique model for studying host-specificity in Colletotrichum. The genome was characterized by potential pathogenic lifestyle-specific expansions and contractions of gene families, particularly those encoding carbohydrate-degrading enzymes. This study indicated that gene family losses were more common than gene family expansions during the evolution of Colletotrichum species. The study also suggested that the ancestor of Colletotrichum already had the genes required and that the observed losses occurred after host specialization. Gan et al. (2016) proposed that the reduced number of myo-inositol transporters in monocot-specific Colletotrichum might have occurred during host specialization. However, this is yet to be explored.

Here, we provide tables with host-specific Colletotrichum species (Table 1) and endemic/native Colletotrichum species (Table 2). In Table 1, we considered the species that even have only one strain and are recorded from one location as host-specific. More collections are needed to confirm whether they are true host-specific species. However, for Table 2, we considered only the species that are recorded from several hosts from a specific country as endemic/native Colletotrichum species.

\section{History of classification}

Corda (1831) introduced the generic name Colletotrichum for $C$. lineola. According to the code of nomenclature, Vermicularia is considered a synonym of Colletotrichum (Duke 1928, Sutton 1992, Damm et al. 2009). Corda $(1837,1840)$ added two more species to the genus. However, this group of fungi became prominent in the $19^{\text {th }}$ century with the publication of Sylloge Fungorium. Between 1880 and 1900, approximately 50 new taxa were described based on the assumption that Colletotrichum species are strongly host-specific, approximately 750 names existed at the time of the first formal monographic treatment (von Arx 1957b, Hyde et al. 2009a, Cannon et al. 2012, Jayawardena et al. 2016a). von Arxs' approach was based on morphological characteristics, which led to the reduction in the accepted number of species from approximately 750 to 11 (within 23 accepted specific and infraspecific taxa). It was clear that he regarded the 11 accepted species as broadly circumscribed aggregates rather than individual taxa. However, von Arxs' approach to classifying Colletotrichum is crude as his evaluations were based on descriptions from the literature rather than on the type material (Sutton 1992, Cannon et al. 2000, 2012). Even so, his work laid the foundation for all subsequent taxonomic work on Colletotrichum.

Table 1 Host-specific Colletotrichum species

\begin{tabular}{llll}
\hline Colletotrichum species & Host & Country & Reference \\
\hline Colletotrichum acidae & Phyllanthus acidus & Thailand & Samarakoon et al. (2018) \\
C. acerbum & Malus domestica & New Zealand & Damm et al. (2012b) \\
C. alatae & Dioscorea alata & Barbardos, & Weir et al. (2012) \\
& & Guadeloupe, India, & \\
C. annellatum & Hevea brasiliensis & Colombia & Damm et al. (2012a) \\
\hline
\end{tabular}


Table 1 Continued.

\begin{tabular}{|c|c|c|c|}
\hline Colletotrichum species & Host & Country & Reference \\
\hline C. anthrisci & Anthriscus sylvestris & Netherlands & Damm et al. (2009) \\
\hline C. antirrhinicola & Antirrhinum majus & New Zealand & Damm et al. (2014) \\
\hline C. arboricola & Fuchsia magellanica & Chile & Crous et al. (2018) \\
\hline C. arecicola & Areca catechu & China & Cao et al. (2020) \\
\hline C. artocarpicola & Artocarpus heterophyllus & Thailand & Bhunjun et al. (2019) \\
\hline C. atractylodicola & Atractylodes chinensis & China & Xu et al. (2018a) \\
\hline C. axonopodi & Axonopus fissifolius & Australia & Crouch et al. (2009) \\
\hline C. baltimorense & Sorghastrum nutans & USA & Crouch $(2014)$ \\
\hline C. beeveri & Brachyglottis repanda & New Zealand & Damm et al. (2012a) \\
\hline C. bidentis & Bidens subalternans & Brazil & Damm et al. (2013) \\
\hline C. bletillum & Bletilla ochracea & China & Tao et al. (2013) \\
\hline C. brasiliense & Passiflora edulis & Brazil & Damm et al. (2012a) \\
\hline C. brassicicola & Brassica oleraceae & New Zealand & Damm et al. (2012a) \\
\hline C. brisbanense & Capsicum апnиum & Australia & Damm et al. (2012b) \\
\hline C. bryoniicola & Bryonia dioica & Netherlands & Damm et al. (2014) \\
\hline C. cacao & Theobroma cacao & Costa Rica & Damm et al. (2019) \\
\hline C. cairnsense & Capsicum anпиum & Australia & de Silva et al. (2017) \\
\hline C. camellia-japonicae & Camellia japonica & Japan & Hou et al. (2016) \\
\hline C. cariniferi & Dendrobium cariniferum & Thailand & Ma et al. (2018) \\
\hline C. catinaense & Citrus sp. & Italy, Portugal & Guarnaccia et al. (2017) \\
\hline C. cattleyicola & Cattleya sp. & Belgium, Japan & Damm et al. (2019) \\
\hline C. caudatum & Sorghastrum nutans & USA & Crouch (2014) \\
\hline C. caudisporum & Bletilla ochracea & China & Tao et al. (2013) \\
\hline C. changpingense & Fragaria $\times$ ananassa & China & Jayawardena et al. (2016b) \\
\hline C. chiangraiense & Dendrobium sp. & Thailand & Ma et al. (2018) \\
\hline C. chrysophilum & Musa sp. & Brazil & Vieira et al. (2017) \\
\hline C. circinans & Allium cepa & Temperate region & $\begin{array}{l}\text { Damm et al. (2009), } \\
\text { Jayawardena et al. (2016a) }\end{array}$ \\
\hline C. citricola & Citrus sp. & China & $\begin{array}{l}\text { Huang et al. (2013), Fu et al. } \\
\text { (2019) }\end{array}$ \\
\hline C. cobbittiense & Cordyline sp. & Australia & Crous et al. (2018) \\
\hline C. coelogynes & Coelogyne sp. & Germany & Damm et al. (2019) \\
\hline C. colombiense & Passiflora edulis & Colombia & Damm et al. (2012a) \\
\hline C. condaoense & Ipomoea psecaprae & Vietnam & Crous et al. (2018) \\
\hline C. cosmic & Cosmos sp. & Netherlands & Damm et al. (2012b) \\
\hline C. costaricense & Coffea $\mathrm{sp}$. & Costa Rica & Damm et al. (2012b) \\
\hline \multirow{2}{*}{ C. curcumae } & Curcuma sp. & India & Damm et al. (2009) \\
\hline & & China & Li et al. (2016) \\
\hline C. cuscutae & Cuscuta sp. & Dominica & Damm et al. (2012b) \\
\hline C. dacrycarpi & Dacrycarpus sp. & New Zealand & Damm et al. (2012a) \\
\hline C. doitungense & Dendrobium sp. & Thailand & Ma et al. (2018) \\
\hline C. dracaenophilum & Dracaena sp. & Bulgaria, China & Damm et al. (2019) \\
\hline C. duyunensis & Bletilla ochracea & China & Tao et al. (2013) \\
\hline C. echinocholae & Echinochola sp. & Japan & Moriwaki \& Tsukiboshi (2009) \\
\hline C. eleusines & Elusines sp. & Japan, USA & Crouch et al. (2009) \\
\hline C. endophytica & Pennisetum purpureum & Thailand & Manamgoda et al. (2013) \\
\hline C. endophytum & Bletilla ochracea & China & Tao et al. (2013) \\
\hline C. eremochloae & Eremochloae ophiuroides & China, USA & $\begin{array}{l}\text { Crouch \& Tomaso-Peterson } \\
\text { (2012) }\end{array}$ \\
\hline C. eriobotryae & Eriobotrya japonica & Taiwan (China) & Damm et al. (2020) \\
\hline C. eryngiicola & Eryngium campestre & Russia & Buyck et al. (2017) \\
\hline C. excelsum-altitudinum & Bletilla ochracea & China & Tao et al. (2013) \\
\hline C. falcatum & Saccharum officinarum & $\begin{array}{l}\text { Australia, } \\
\text { Indonesia, Japan, } \\
\text { Netherlands, } \\
\text { Thailand }\end{array}$ & Prihastuti et al. (2010) \\
\hline C. feijoicola & Acca sellowiana & Portugal & Crous et al. (2019) \\
\hline C. graminicola & Zea mays & Worldwide & Farr \& Rossman (2021) \\
\hline C. grevilleae & Grevillea sp. & Italy & Liu et al. (2013) \\
\hline
\end{tabular}


Table 1 Continued.

\begin{tabular}{|c|c|c|c|}
\hline Colletotrichum species & Host & Country & Reference \\
\hline C. guizhouensis & Bletilla ochracea & China & Tao et al. (2013) \\
\hline C. hanaui & Digitaria ciliaris & China, Japan, USA & Crouch et al. (2009) \\
\hline C. hainanense & Axonopus compressus & China & Zhang et al. (2020) \\
\hline C. hebeiense & Vitis vinifera & China & Yan et al. (2015) \\
\hline C. hederiicola & Hedera helix & Italy & Hyde et al. (2020) \\
\hline C. hemerocallidis & Hemerocallis fulva & Canada, China & Yang et al. (2012a) \\
\hline C. higginsianum & genera of Brassicaceae & Worldwide & Damm et al. (2014) \\
\hline C. hippeastri & Hippeastrum sp. & China, Netherland & Damm et al. (2012a) \\
\hline C. horii & Diospyros kaki & $\begin{array}{l}\text { Brazil, China, } \\
\text { Japan, Korea, New } \\
\text { Zealand, South } \\
\text { Korea }\end{array}$ & Farr \& Rossman (2021) \\
\hline C. hsienjenchang & Phyllostachys & Japan & Sato et al. (2012) \\
\hline C. hystricis & Citrus hystrix & Italy & Guarnaccia et al. (2017) \\
\hline C. incanum & Glycine max & USA & Yang et al. (2014) \\
\hline C. indonesiense & Eucalyptus sp. & Indonesia & Damm et al. (2012b) \\
\hline C. insertae & Parthenocissus inserta & Russia & Hyde et al. (2016) \\
\hline C. jacksonii & Echinochloa esculenta & Japan & Crouch et al. (2009) \\
\hline C. jasminigenum & Jasminum sambac & Vietnam & Wikee et al. (2011) \\
\hline C. javanense & Capsicum аппиит & Indonesia & de Silva et al. (2019) \\
\hline C. jiangxiense & Camellia sinensis & China & Liu et al. (2015) \\
\hline C. jinshuiense & Pyrus pyrifolia & China & Fu et al. (2019) \\
\hline C. jishouense & Nothapodytes pittosporoides & China & Zhou et al. (2019) \\
\hline C. kakivorum & Diospyrus kaki & Korea & Lee \& Jung (2018) \\
\hline C. kinghornii & Phormium sp. & UK & Damm et al. (2012b) \\
\hline C. laticiphilum & Hevea brasiliensis & $\begin{array}{l}\text { Inida, Colombia, } \\
\text { Sri Lanka, China }\end{array}$ & $\begin{array}{l}\text { Damm et al. (2012b) } \\
\text { Hunupolagama et al. (2017) } \\
\text { Shi et al. (2019) }\end{array}$ \\
\hline C. lauri & Laurus nobilis & Italy & Hyde et al. (2017) \\
\hline C. lilii & Lillium sp. & Japan & Damm et al. (2009) \\
\hline C. ledebouriae & Ledebouria floridunda & South Africa & Crous et al. (2016) \\
\hline C. liaoningense & Capsicum аппиит & China & Diao et al. (2017) \\
\hline C. limonicola & Citrus limon & Malta & Guarnaccia et al. (2017) \\
\hline C. lindemuthianum & Phaseolus sp. & Worldwide & Jayawardena et al. (2016a) \\
\hline C. magnum & Citrullus lanatus & USA & Damm et al. (2019) \\
\hline C. malvarum & Genera of Malvaceae & $\begin{array}{l}\text { Germany, Korea, } \\
\text { UK }\end{array}$ & Damm et al. (2013) \\
\hline C. makassarense & Capsicum anпuит & Indonesia & de Silva et al. (2019) \\
\hline C. menispermi & Menispermum dauricum & Russia & Li et al. (2016) \\
\hline C. merremiae & Merremia umbellata & Panama & Damm et al. (2019) \\
\hline C. musae & Musa sp. & Worldwide & Weir et al. (2012) \\
\hline C. navitas & Panicum sp. & USA & Crouch et al. (2009b) \\
\hline C. neosansevieriae & Sansevieria trifasciata & South Africa & Crous et al. (2015) \\
\hline C. nicholsonii & Paspalum dilatatum & $\begin{array}{l}\text { Japan, New } \\
\text { Zealand }\end{array}$ & Crouch et al. (2009a) \\
\hline C. ocimi & Ocimum basilicum & $\begin{array}{l}\text { Italy } \\
\text { Australia }\end{array}$ & $\begin{array}{l}\text { Damm et al. (2014) } \\
\text { Shivas et al. (2016) }\end{array}$ \\
\hline C. orchidis & Orchis sp. & Italy & Hyde et al. (2020) \\
\hline C. okinawense & Carica papaya & $\begin{array}{l}\text { Japan } \\
\text { Brazil }\end{array}$ & $\begin{array}{l}\text { Damm et al. (2019) } \\
\text { Dias et al. (2020) }\end{array}$ \\
\hline C. ochraceae & Bletilla ochracea & China & Tao et al. (2013) \\
\hline C. oncidii & Oncidium sp. & Germany & Damm et al. (2012a) \\
\hline C. orbiculare & genera of Cucubitaceae & Worldwide & $\begin{array}{l}\text { Damm et al. (2013), Farr \& } \\
\text { Rossman (2021) }\end{array}$ \\
\hline C. orchidophilum & Orchidaceae & $\begin{array}{l}\text { Panama, UK, USA, } \\
\text { France }\end{array}$ & $\begin{array}{l}\text { Damm et al. (2012b), Charron et } \\
\text { al. (2018) }\end{array}$ \\
\hline C. panacicola & Panax sp. & $\begin{array}{l}\text { China, Japan, } \\
\text { Korea, Russia }\end{array}$ & Damm et al. (2014) \\
\hline C. panamense & Merremia umbellate & Panama & Damm et al. (2019) \\
\hline
\end{tabular}


Table 1 Continued.

\begin{tabular}{|c|c|c|c|}
\hline Colletotrichum species & Host & Country & Reference \\
\hline C. parallelophorum & Dendrobium sp. & Thailand & Ma et al. (2018) \\
\hline C. parthenocissicola & Parthenocissus quinquefolia & Russia & Yuan et al. (2020) \\
\hline C. paspali & Paspalum notatum & Japan & Crouch et al. (2009a) \\
\hline C. pandanicola & Pandanus sp. & Thailand & Tibpromma et al. (2018) \\
\hline C. paxtonii & Musa sp. & West Indies & Damm et al. (2012b) \\
\hline C. persea & Persea americana & Israel & Sharma et al. (2017) \\
\hline C. petchii & Dracaena sp. & $\begin{array}{l}\text { China, Germany, } \\
\text { Italy, Netherlands, } \\
\text { Sri Lanka, Australia }\end{array}$ & $\begin{array}{l}\text { Damm et al. (2012a), Shivas et } \\
\text { al. (2016) }\end{array}$ \\
\hline C. phormii & Phormium sp. & $\begin{array}{l}\text { Germany, } \\
\text { Netherlands, New } \\
\text { Zealand, South } \\
\text { Africa, UK }\end{array}$ & $\begin{array}{l}\text { Farr et al. (2006), Damm et al. } \\
\text { (2012b), Serdani et al. (2013) }\end{array}$ \\
\hline C. piperis & Piper nigrum & Malaysia & Damm et al. (2019) \\
\hline C. pisicola & Pisum sp. & $\begin{array}{l}\text { Ecuador, Mexico, } \\
\text { USA }\end{array}$ & Damm et al. (2014) \\
\hline C. pseudomajus & Camellia sinensis & Taiwan & Liu et al. (2014) \\
\hline C. pseudotheobromicola & Prunus avium & China & Chethana et al. (2019) \\
\hline C. psidii & Psidium sp. & Italy & Weir et al. (2012) \\
\hline C. protea & Protea sp. & South Africa & Liu et al. (2013) \\
\hline C. pyrifoliae & Pyrus pyrifolia & China & Fu et al. (2019) \\
\hline C. quinquefoliae & Parthenocissus quinquefolia & Russia & Li et al. (2016) \\
\hline C. riograndense & Tradescantia fluminensis & Brazil & Macedo et al. (2016) \\
\hline C. salsolae & Salsola tragus & Hungary & Weir et al. (2012) \\
\hline C. sambucicola & Sambucus ebulus & Italy & Tibpromma et al. (2017) \\
\hline \multirow{4}{*}{ C. sansevieriae } & Sansevieria sp. & Australia & Aldaoud et al. (2011) \\
\hline & & Japan & Nakamura et al. (2006) \\
\hline & & India & Gautam et al. (2012) \\
\hline & & Iran & Karimi et al. (2017) \\
\hline C. sedi & Sedum sp. & Russia & Liu et al. (2015) \\
\hline C. serranegrense & Cattleya jongheana & Brazil & Silva et al. (2018) \\
\hline C. shisoi & Perilla frutescens & Japan & Gan et al. (2019) \\
\hline C. sidae & Sida spinosa & USA & Damm et al. (2013) \\
\hline C. somersetense & Sorghastrum nutans & USA & Crouch (2014) \\
\hline C. sonchicola & Sonchus sp. & Italy & Jayawardena et al. (2017) \\
\hline C. spinosum & Xanthium spinosum & $\begin{array}{l}\text { Argentina, } \\
\text { Australia }\end{array}$ & Damm et al. (2013) \\
\hline C. sublineola & Sorghum sp. & Togo, USA & $\begin{array}{l}\text { Crouch \& Tomaso-Peterson } \\
\text { (2012) }\end{array}$ \\
\hline C. sydowii & Sambucus sp. & Taiwan (China) & Marlin-Felix et al. (2017) \\
\hline C. syzygicola & $\begin{array}{l}\text { Citrus aurantifolia, Syzygium } \\
\text { samarangense }\end{array}$ & Thailand & Udayanga et al. (2013) \\
\hline C. tanaceti & Tanacetum cinerariifolium & Australia, Tasmania & Damm et al. (2014) \\
\hline C. tainanense & Capsicum annuum & Taiwan & de Silva et al. (2019) \\
\hline C. tebeestii & Malva pustilla & Canada & Damm et al. (2013) \\
\hline C. temperatum & Vaccinium macrocarpon & USA & Doyle et al. (2013) \\
\hline C. $t i$ & Cordyline sp. & New Zealand & Weir et al. (2012) \\
\hline C. tongrenense & Nothapodytes pittosporoides & China & Zhou et al. (2019) \\
\hline C. trichellum & Hederea sp. & $\begin{array}{l}\text { Canada, Germany, } \\
\text { Guatemala, } \\
\text { Netherlands, New } \\
\text { Zealand, UK }\end{array}$ & Damm et al. (2009) \\
\hline C. utrechtense & Trifolium pratense & Netherlands & Damm et al. (2014) \\
\hline C. verruculosum & Crotalaria juncea & Zimbabwe & Damm et al. (2009) \\
\hline C. vietnamense & Coffea sp. & Vietnam & Liu et al. (2014) \\
\hline C. vignae & Vigna unguiculata & Nigeria & Damm et al. (2014) \\
\hline \multirow[t]{2}{*}{ C. viniferum } & Vitis vinifera & China & Peng et al. (2013) \\
\hline & & South Korea & Oo \& Oh (2017) \\
\hline
\end{tabular}


Table 1 Continued.

\begin{tabular}{llll}
\hline Colletotrichum species & Host & Country & Reference \\
\hline C. walleri & Coffea sp. & Vietnam & Damm et al. (2012b) \\
C. wanningense & Hevea brasiliensis & China & Cao et al. (2019) \\
C. watpharaense & Dendrobium sp. & Thailand & Ma et al. (2018) \\
C. wuxiense & Camellia sinensis & China & Wang et al. (2016) \\
C. xanthorrhaea & Xanthorrhoea preissii & Australia & Shivas et al. (2009) \\
C. yulongense & Vaccinium dunalianum & China & Wang et al. (2019) \\
C. yunnanense & Buxus sp. & China & Liu et al. (2007) \\
C. zoysiae & Zoysia tenuifolia & Japan & Crouch (2014) \\
\hline
\end{tabular}

Table 2 Endemic/Native Colletotrichum species

\begin{tabular}{|c|c|c|c|}
\hline Colletotrichum species & Country & Host & Reference \\
\hline Colletotrichum alcornii & Australia & $\begin{array}{l}\text { Bothriochloa bladhii, Imperata } \\
\text { cylindrical }\end{array}$ & Crouch (2014) \\
\hline C. aotearoa & New Zealand & $\begin{array}{l}\text { Banksia marginata, Boehmeria } \\
\text { sp., Bredia oldhamii, Coprosma } \\
\text { sp., Dacrycarpus dacrydioides, } \\
\text { Knightia sp., Musa } \text { sp., } \\
\text { Podocarpus totara, Vitex lucens }\end{array}$ & Weir et al. (2012) \\
\hline \multirow[t]{2}{*}{ C. carthami } & \multirow[t]{2}{*}{ Japan } & Carthamus tinctorius & Uematsu et al. (2012) \\
\hline & & Calendula officinalis & Baroncelli et al. (2015a) \\
\hline C. constrictum & New Zealand & Citrus limon, Solanum betacum & Damm et al. (2012a) \\
\hline C. fructi & USA & Malus sylvestris, Pyrus malus & Damm et al. (2009) \\
\hline C. helleniense & Greece & $\begin{array}{l}\text { Citrus reticulata, Poncirus } \\
\text { trifoliate }\end{array}$ & Guarnaccia et al. (2017) \\
\hline \multirow[t]{2}{*}{ C. henanense } & \multirow[t]{2}{*}{ China } & $\begin{array}{l}\text { Camellia sinensis, Cirsium } \\
\text { japonicum }\end{array}$ & Liu et al. (2015) \\
\hline & & Camellia oleifera & Li et al. (2018) \\
\hline \multirow[t]{2}{*}{ C. guajavae } & \multirow[t]{2}{*}{ India } & Psidium guajava & Damm et al. (2012b) \\
\hline & & Elettaria cardamomum & Chethana et al. (2016) \\
\hline C. johnstonii & New Zealand & $\begin{array}{l}\text { Solanum lycopersicum } \\
\text { Citrus sp. }\end{array}$ & Damm et al. (2012b) \\
\hline C. kahawae & African continent & Coffea Arabica & $\begin{array}{l}\text { Waller et al. (1993), Weir et al. } \\
\text { (2012) }\end{array}$ \\
\hline C. neosansevieriae & South Africa & Sansevieria trifasciata & Crous et al. (2015) \\
\hline C. nupharicola & USA & Nuphar, Nymphae & Johnston et al. (1997) \\
\hline \multirow[t]{2}{*}{ C. phyllanthi } & \multirow[t]{2}{*}{ India } & Phyllanthus acidus & Damm et al. (2012a) \\
\hline & & $\begin{array}{l}\text { Bauhinia variegate, Bougainvillea } \\
\text { glabra }\end{array}$ & Sharma \& Shenoy (2013) \\
\hline C. rhexiae & USA & $\begin{array}{l}\text { Rhexia virginica, Vaccinium } \\
\text { macrocarpon }\end{array}$ & Doyle et al. (2013) \\
\hline \multirow[t]{2}{*}{ C. tamarolii } & \multirow[t]{2}{*}{ Colombia } & Solanum betaceum (anthracnose) & Damm et al. (2012b) \\
\hline & & Cyphomandra betacea & Baroncelli et al. (2015b) \\
\hline C. torulosum & New Zealand & $\begin{array}{l}\text { Passiflora edulis, Solanum } \\
\text { melongena }\end{array}$ & Damm et al. (2012a) \\
\hline C. trifolii & USA & Medicago sp., Trifolium sp. & Damm et al. (2013) \\
\hline C. vittalense & India & $\begin{array}{l}\text { Calamus thwaitesii, Orchid sp., } \\
\text { Theobroma cacao }\end{array}$ & Damm et al. (2019) \\
\hline
\end{tabular}

Subsequent taxonomic treatments mainly focused on species or taxa associated with a particular crop. Simmonds (1965) recognized C. acutatum, Sutton $(1966,1968)$ made contributions in the taxonomy of graminicola complex and understanding the value of appressorial morphology in classification. Sutton (1980) provided the next comprehensive treatment, accepting 22 species. Baxter et al. (1983) contributed 11 South African species. Both of these studies mainly relied on morphological and cultural characteristics. Smith \& Black (1990) identified the species associated with strawberry, while Walker et al. (1991) identified species associated with Xanthium based on 
the integration of taxonomy and pathological data. The first international workshop on Colletotrichum was held in late 1990 in the UK (Bailey \& Jeger 1992) and marked the dawn of using molecular methods in Colletotrichum studies. Initial studies focused on intraspecific variation (Dale et al. 1988, Braithwaite \& Manners 1989, Braithwaite et al. 1990a, b, Masel et al. 1990). Mills et al. (1992) published the first applications of DNA sequence data to distinguish between Colletotrichum species. Sreenivasaprasad et al. (1992) identified sequence variation in the ITS1 region of nrDNA between six species of Colletotrichum. This study also detected polymorphisms in the same region between strains of $C$. gloeosporioides from different hosts. Sherriff et al. (1994) presented the first bootstrapped neighbour-joining tree for Colletotrichum using ITS2 and LSU sequence data of 27 strains, resulting in 13 species. In that study, $C$. orbiculare was identified as a different taxonomic unit and genetic congruence was detected between the four curved-spored species studied. Sreenivasaprasad et al. (1996) using parsimony analysis of ITS1 and ITS2 sequences from 18 Colletotrichum species published the second phylogenetic study of the genus, which resulted in identifying six infrageneric groups. Talhinhas et al. (2002) published the first multilocus phylogenetic analyses of Colletotrichum species as a study of the C. acutatum complex associated with lupins using ITS, tub2 and his 34 sequence data. Vinnere et al. (2002) provided a multilocus phylogenetic analysis using ITS, $t u b 2$ and $\mathrm{mtSSU}$ sequence data in a study on the same species complex associated with Rhododendron in Sweden and Latvia. Talhinhas et al. (2002) found that the three loci they studied displayed broadly similar levels of phylogenetic resolution. Guerber et al. (2003) used glyceraldehyde-3-phosphate dehydrogenase (gapdh) and glutamine synthetase $(g s)$ nucleotide sequence data in their study of the $C$. acutatum complex. Du et al. (2005) found that the HMG-box section of the mating-type genes mat- 1 was a valuable genetic marker for this genus. From around this time, multilocus analyses became the norm as sequencing costs reduced, with sequence data generated from different loci (Table 3).

Table 3 Different gene loci used in Colletotrichum identification

\begin{tabular}{ll}
\hline Name & Reference \\
\hline ITS & Mills et al. (1992) \\
LSU & Sherriff et al. (1994) \\
mat2 & Chen et al. (2002) \\
tub2 & Talhinhas et al. (2002) \\
his34 & Talhinhas et al. (2002) \\
mtSSU & Vinnere et al. (2002) \\
gapdh & Guerber et al. (2003) \\
gs & Guerber et al. (2003) \\
mat 1-2 (HMG marker) & Du et al. (2005) \\
sod2 & Crouch et al. (2006) \\
Ccret2 & Crouch et al. (2008) \\
apn2/Igs/mat1-2 & Crouch et al. (2009) \\
act & Damm et al. (2009) \\
chs-1 & Damm et al. (2009) \\
cal & Prihastuti et al. (2009) \\
ef1 $\alpha$ & Rojas et al. (2010) \\
rpb1 & Rojas et al. (2010) \\
apmat & Silva et al. (2012a, b) \\
apn151 & Silva et al. (2012a, b) \\
mat5L & Silva et al. (2012a, b) \\
apn1ex3 & Silva et al. (2012a, b) \\
apn131 & Silva et al. (2012a, b) \\
\hline
\end{tabular}

The special issue of the journal Fungal Diversity in 2009, containing a group of papers presenting taxonomic revisions and review articles relevant to the genus, was a milestone in Colletotrichum taxonomy. Hyde et al. (2009a) accepted 66 species with an additional 20 names considered as doubtful. In his study, he highlighted the importance of using polyphasic approaches. Cannon et al. (2012) accepted 118 species based on morphological and phylogenetic data. 
Jayawardena et al. (2016a) accepted 190 species with molecular data, identified one doubtful species, and excluded one species. Many revisions have since been published on the genus based on multi-locus sequence data (Damm et al. 2009, 2012a, b, 2013, 2014, 2019, Weir et al. 2012, Crouch 2014, Hyde et al. 2014, Bhunjun et al. 2021, Jayawardena et al. 2020). An updated backbone diagrammatic tree for the genus is given in Fig. 3.

Studies on Colletotrichum species from forest plants or plants in natural/semi-natural habitats have been much less studied (Hyde et al. 2020). There is a need to expand study of Colletotrichum beyond economically important plants, as these may act as a reservoir of common and novel species, thus providing a better overall understanding of the genus.

\section{Various methods in defining Colletotrichum species}

Currently, species delimitation in Colletotrichum relies solely on the use of multi locus phylogenetic analyses (Cannon et al. 2012, Hyde et al. 2014, Jayawardena et al. 2020). However, different methods can be used to provide better species delimitation.

\section{Genealogical concordance phylogenetic species recognition (GCPSR)}

This concept was proposed as an empirical method for recognizing cryptic speciation (Taylor et al. 2000). The GCPSR involves sequencing multiple genes that are then combined in phylogenetic analysis (Nguyen et al. 2015). The point of genetic isolation is identified through incongruent nodes and therefore interpreted as the species limit or the point of genetic isolation (Dettman et al. 2003, O’Donnell et al. 2008, Henk et al. 2011, Leavitt et al. 2011). This concept is important for delimitation of morphologically similar species that only exhibit their asexual morphs (Nguyen et al. 2015). Genealogical concordance phylogenetic species recognition considers phylogenetic trees of different genes that show discordance within a species due to gene flow between individuals (Liu et al. 2016c). According to the GCPSR criteria, the lack of genealogical congruence among gene trees is a signal that the sampled diversity is below species level (Taylor et al. 2000). In contrast, concordance between gene trees is strong evidence for the distinct and congruent clades to represent reproductively isolated lineages.

Colletotrichum gloeosporioides is a species complex with broad genetic and biological diversity, whose numbers have similar conidial morphology and ITS sequences (Damm et al. 2010). Weir et al. (2012) used the GCPSR concept to delimit taxa within the gloeosporioides species complex. They applied GCPSR with eight genes using the Bayesian analysis tool, BUCKy (Ané et al. 2007). The GCPSR concept worked well for species delimitation along currently recognised lines. However, C. kahawae, was insufficiently distinct from several genetically similar non-coffee berry disease-causing taxa (Weir et al. 2012). In their study, they suggested that this may be due to the recent emergence of $C$. kahawae as a pathogen and that insufficient time had passed for ecological niche specialisation to show polymorphisms in the genes used. Therefore, C. kahawae was recognised at the subspecific rank. In Weir et al. (2012), not all within species phylogenetic structures supported by multiloci phylogeny were resolved consistently in all gene trees.

Liu et al. (2016d) utilized the GCPSR concept to test the null hypothesis that ' $C$. siamense is a species complex'. In their study they pointed out that supported nodes in a single gene tree might conflict with those in the concatenated multi-locus tree, as well as in other single gene trees. The combination of multiple loci, which separately do not support a clade, can often reveal support for or conflict within that clade (Gatesy \& Baker 2005). In the phylogenetic analyses of $C$. siamense sensu lato, conflicts were reported between any pair of single locus phylograms, or even in concatenated gene trees (Liu et al. 2016c). Therefore, implementing GCPSR criteria lead to the rejection of the null hypothesis. The topology of the apmat phylogram was almost congruent with that of the 8-locus phylogram (Liu et al. 2016c). Mating-related genes evolve at a faster rate and have a higher sequence variability, which therefore dominates the topology of the multi-locus phylogram (Silva et al. 2012a, b). It should be noted that single-locus data inferred the evolutionary history of a single gene, but not that of the organisms (Frantz et al. 2013). 


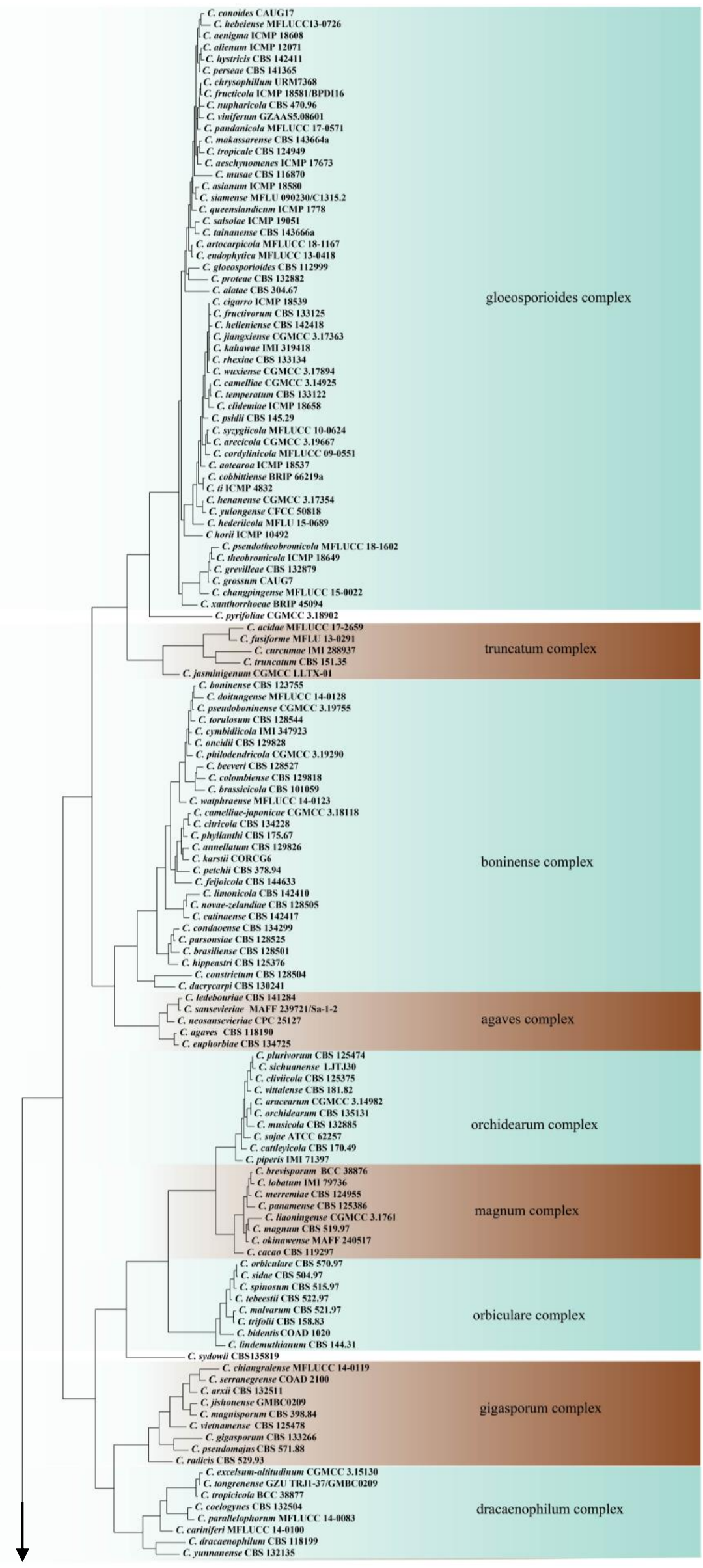

Figure 3 - Backbone diagrammatic tree of Colletotrichum. One of the 100 most parsimonious trees 
obtained from a heuristic search of combined ITS, gapdh, chs-1, act and tub2 sequence data. The tree is rooted with Monilochaetes camelliae (BRIP 24607) and M. infuscans (CBS 869.96).

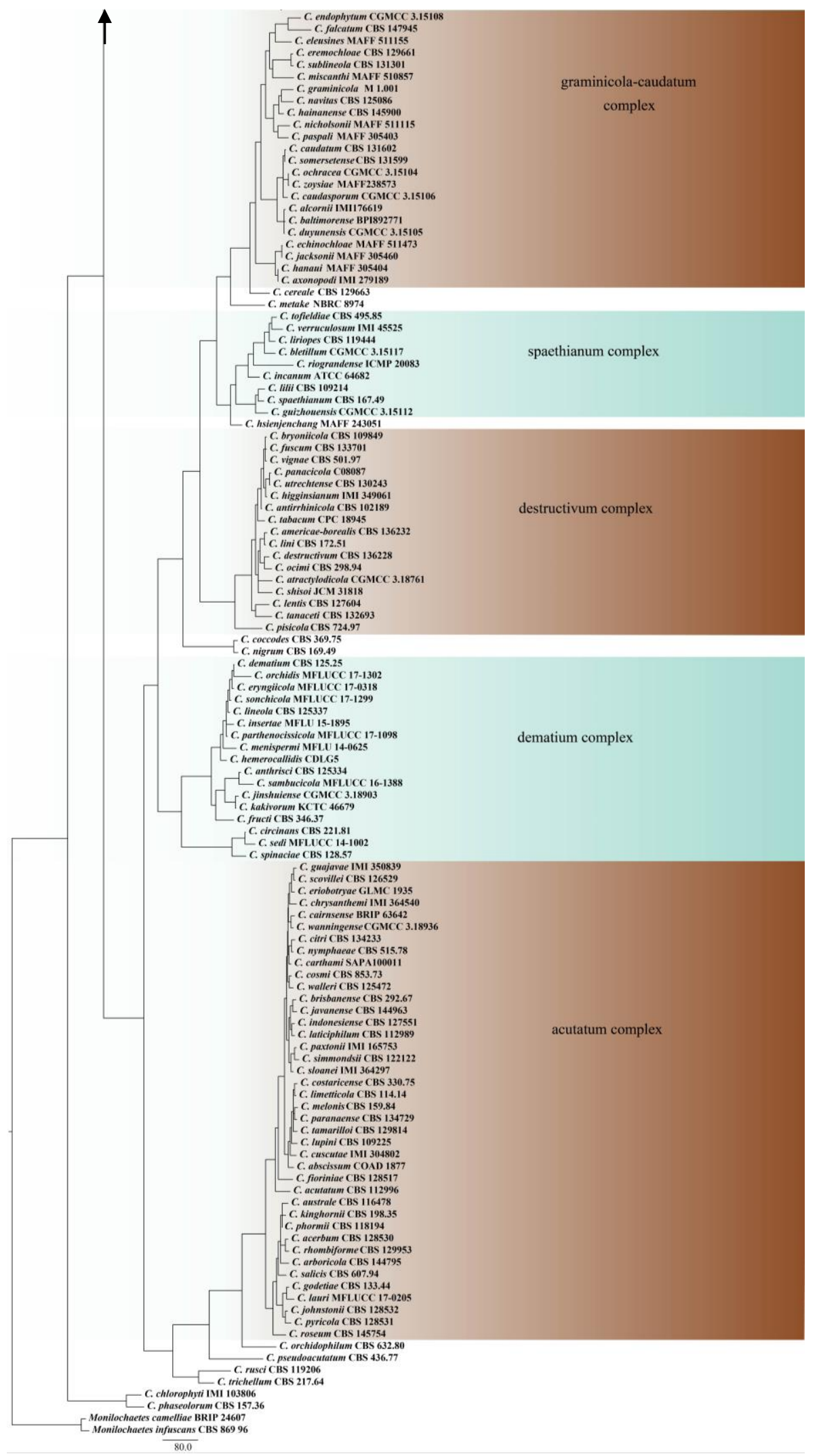

Figure 3 - Continued.

Cavalcante et al. (2019) considered a clade as an independent lineage when it met at least one of the two criteria: genealogical concordance or genealogical nondiscordance. Genealogical 
concordance criterion is satisfied, if the clade is present in most individual gene trees. Genealogical nondiscordance criterion is satisfied when a clade is strongly supported by at least one locus in more than one phylogenetic analyses (ML and BYPP $\geq 70 \%$ and $\geq 0.95$ respectively), and not contradicted in any other individual gene tree with the same level of support. Based on these GCPSR criteria, Cavalcante et al. (2019) assigned the Colletotrichum sp. associated with lima bean in Brazil to four species: C. brevisporum, C. lobatum, C. magnum and C. truncatum and no discordance was observed among individual gene trees.

\section{Pairwise homoplasy index test (PHI or Фw)}

Recombination is a powerful evolutionary force that merges historically distinct genotypes. However, the extent of recombination within many organisms is unknown, and even determining its presence within a set of homologous sequences is a difficult task (Awadalla 2003). Tests for recombination based on the principle of compatibility are amongst the most powerful tools in species identification (Brown et al. 2001, Posada 2002). Bruen et al. (2006) developed a new statistical test for recombination, the PHI statistic that uses this notion of refined incompatibility. Pairwise homoplasy index test takes into account convergent or recurrent mutations (homoplasies). Sites are compared and deemed compatible, if there are no homoplasies. The presence of homoplasies indicates incompatible sites and recombination.

The GCPSR as mentioned earlier is a pragmatic tool for the assessment of species limits, as the concordance of gene genealogies is a valuable criterion for evaluating the significance of gene flow between groups within an evolutionary timescale (Quaedvlieg et al. 2014). Many studies have conducted the PHI test using the GCPSR model to determine the recombination level between taxa when describing new Colletotrichum species (Crouch 2014, Liu et al. 2015a, 2016d, Jayawardena et al. 2016a, 2017, Bhunjun et al. 2019). Results of PHI below a 0.05 threshold $\left(\Phi_{\mathrm{W}}<0.05\right)$ indicate the presence of significant recombination. This means that the species under consideration cannot be different species.

\section{Haplotype and Phylogenetic network analysis}

Phylogenetic network analysis takes reticulation events into account (such as hybridization, recombination and/or horizontal gene transfer) (Huson \& Scornavacca 2011). Rogério et al. (2017) studied 51 strains of $C$. truncatum from soybean in Brazil and identified the existence of a highly efficient mechanism of pathogen dispersal over long distances, reinforcing the role of seeds as the primary source of disease inoculum. Based on this approach, Liu et al. (2016d) provided evidence to synonymise seven species in the siamense complex to $C$. siamense. Douanla-Meli et al. (2018) identified that $C$. cliviicola isolates of each geographic region were not always resolved into separate groups. The first group included the most geographically diversified group, composed of isolates from Brazil, China, Mexico and Vietnam. The second group contained isolates from Cameroon, South America (Brazil, Puerto Rico) and Vietnam. There was another group made up exclusively of isolates from different hosts in China. Crouch et al. (2008) used a four-gene nucleotide data set to diagnose the limits of population boundaries. Colletotrichum cereale was divided into ten highly specialized populations corresponding to the ecosystem and/or host plant, along with a single generalist population spread across multiple habitat types (Crouch et al. 2008).

\section{Coalescent-based species delimitation (CBD)}

In multi-locus analyses, incongruence between gene trees and species trees is commonly detected. Incomplete lineage is considered a common source of discordance (Kubatko et al. 2011). Incomplete lineage sorting refers to gene copies retaining ancestral polymorphisms thus resulting in discordant gene trees (Hudson \& Coyne 2002). Coalescent-based delimitation involves modelling the genealogical history of samples (e.g. loci) to a common ancestor (Fujita et al. 2012). Coalescent-based species delimitation has been used in discovering a large number of cryptic species in animals and plants (Waters et al. 2010, Stewart et al. 2014). However, these approaches are seldomly applied in fungi, especially in a complex group of plant pathogens like 
Colletotrichum. Liu et al. (2016d) applied several coalescent-based species delimitation methods to infer species boundaries of the 'siamense species complex'. Douanla-Meli et al. (2018) applied CBD to identify the relationship among $C$. cliviae (now known as $C$. cliviicola), $C$. sichuanensis and $C$. orchidearum. Based on this analysis, $C$. sichuanensis was synonimized under C. cliviicola. Bhunjun et al. (2021) used CBD methods to elucidate species and species complex boundaries within Colletotrichum. Based on their findings, the sansevieriae species complex was introduced and they provided recommendations for species boundaries in the graminicola-caudatum complex.

\section{General Mixed Yule Coalescent (GMYC) approach}

The GMYC approach is designed for single-locus analysis however, it has been used for multilocus phylogeny as well (Arrigoni et al. 2016). This approach combines the neutral coalescent theory with the Yule speciation model and aims at detecting shifts in branching rates between intraand interspecific relationships. This method requires an ultrametric tree. Liu et al. (2016d) used GMYC approach when defining the species boundaries for 'siamense species complex'. In their analyses, all the strains used in the analyses which were identified as different species were recognized as a single species. Bhunjun et al. (2021) used GMYC approach for the whole genus which detected 97 entities based on the multi-locus dataset and all the singletons were recovered as conspecific with few exceptions.

\section{Poisson Tree Processes (PTP) model}

This method is designed for single-locus analysis, however, it has been used for multilocus phylogeny (Renner et al. 2017). The PTP method directly estimates the mean expected number of substitutions per site between two branching events. It uses the tree branch length information and then implements two independent classes of Poisson processes (intra and inter-specific branching events). This method only requires a likelihood tree. Liu et al. (2016d) used PTP model to provide more support for treating Colletotrichum siamense as a single species, not as a cryptic species. In Bhunjun et al. (2021) 26 entities were recovered for the whole genus based on multiloci data set.

\section{Species validation}

This method accommodates the species phylogeny as well as incomplete lineage sorting due to ancestral polymorphisms (Yang \& Rannala 2010). Its use has several advantages over other alternatives and is commonly used for species delimitation (Fujita \& Leache 2011). Bayesian phylogenetics and phylogeography incorporate a nearest-neighbour interchange algorithm allowing changes in the species tree topology and eliminating the need for a fixed user-specified guide tree (Yang \& Rannala 2010).

\section{Molecular clock studies}

These types of studies can provide details on how species of Colletotrichum evolved with their hosts in nature. Only few studies have identified the examined these aspects of Colletotrichum.

Hacquard et al. (2016) estimated the divergence time of Colletotrichum based on a penalized likelihood method of genome sequence data using the crown age of Pezizomycotina, Sordariomycetes and Cordyceps-Metarhizium divergence as calibration points. They estimated that C. incanum and C. tofieldiae diverged approximately 8.8 MYA.

Liang et al. (2018) estimated the divergence time of several complexes based on a penalized likelihood method of genome sequence data using the crown age of Colletotrichum, Sordariomycetes, and Sordariomycetes-Leotiomycetes crown as the calibration points and the estimates were based on four calibration schemes. According to this study, C. fructicola and C. gloeosporioides (gloeosporioides complex) diverged approximately 3.8-5.1 MYA, C. graminicola and C. sublineola (graminicola complex) diverged approximately 11-15 MYA,

C. incanum and C. tofieldiae (spaethianum complex) diverged approximately 7.5-11 MYA, C. salicis, C. fioriniae, C. nymphaeae and C. simmondsii (acutatum complex) diverged 
approximately 8-10.9 MYA. The most recent common ancestor (MRCA) of the acutatum, graminicola, spaethianum and destructivum complex was estimated at 37-49 MYA.

Lelwala et al. (2019) estimated the divergence time based on a penalized likelihood method of genome sequence data using the crown age of Leotiomycetes-Sordariomycetes, crown age of Sordariomycetes and Colletotrichum crown as the calibration points. Colletotrichum higginsianum and $C$. tanaceti (destructivum complex) diverged around 9.9 MYA, C. fructicola and C. gloeosporioides (gloeosporioides complex) diverged around 6.1 MYA, C. graminicola and C. sublineola (graminicola complex) diverged around $15.8 \mathrm{MYA}, C$. fioriniae, C. nymphaeae, C. salicis and C. simmondsii (acutatum complex) diverged around 10.9 MYA. The MRCA of the acutatum, graminicola, spaethianum and destructivum complex was estimated at 48.9 MYA. The MRCA of the graminicola, destructivum and spaethianum complex was estimated at 34.7 MYA.

Bhunjun et al. (2021) used all species in Colletotrichum and provided an estimated divergence time for all the complexes. The estimated crown age of Colletotrichum was 61-63 MYA. In his study, three singleton taxa claded in species complexes. Colletotrichum axonopodi was closely related to $C$. hanaui in the graminicola complex whereas $C$. parallelophorum and C. cariniferi claded in the dracaenophilum complex. The study strongly supported a new species complex, which comprised $C$. agaves, $C$. euphorbiae, C. ledebouriae, C. neosansevieriae and C. sansevieriae with an estimated divergence time of 9.5 MYA.

Further molecular clock studies are needed to understand the host association of Colletotrichum species.

\section{Are we introducing too many species?}

\section{Ignorance of morphospecies}

Every year many species are introduced in this genus (see Fig. 4). Are we introducing species that have been introduced in earlier studies that lack molecular data? As listed in Table 19 there are 450 morphospecies. If mycologists introduce new species on a host, for which there are previous records of morphospecies (species without molecular data), it is important to check the morphological descriptions to avoid this problem. For example, if a new species is introduced on rubber (Hevea sp.), the morphology of the species should be compared with $C$. heveae Petch. However, in some cases, the original descriptions of the morphospecies and type species are not available. Sometimes, a species is considered invalid by Index Fungorum, while it is accepted as a valid species elsewhere (e.g. C. rubicola). As this is very confusing, we recommend epitypification/neotypification of these species.

\section{Taxon sampling}

Taxon sampling is important when we are introducing a new species in this genus. Wrong identification or misinterpretation can occur based on the number of taxa that we use in phylogenetic analyses (Young \& Gillung 2020). Theoretically, the phylogenetic species concept is based on the assumption that 'the fixation of a particular character state in a population is diagnostic of a long history of reproductive isolation (Liu et al. 2016c). However, in practice, species recognition is carried out based on the characters of a small group of individuals. Unfortunately, within a particular species, a small sample size of individuals in a population might share one unique character, which can be polymorphic. This leads to the problem that using one or only a few individuals does not capture the intra-specific diversity of a species (Davis \& Nixson 1992, Walsh 2000, Liu et al. 2016c). New species might be mistakenly described if two divergent populations present certain morphological or genetic distinctions (Goldstein et al. 2000). Colletotrichum being widely distributed and with little morphological diversity faces the problem of wrong identification when using the phylogenetic species concepts. One of the best examples is given in Liu et al. (2016). Colletotrichum endomangiferae (Viera et al. 2014), C. hymenocallidis (Yang et al. 2009), C. jasmini-sambac (Wikee et al. 2011) and C. melanocaulon (Doyle et al. 2013) were introduced as new species in the $C$. siamense species complex. Sharma et al. (2015) also 
accepted these as separate species in the siamense complex. However, Liu et al. (2016d) showed that all of the above-mentioned species were introduced based on two or three strains. Hence, by adding more strains, the analyses showed that the four species were in fact $C$. siamense. Colletotrichum aciculare was introduced in Ariyawansa et al. (2015) based on one strain. According to the phylogenetic analyses, it clustered in the truncatum species complex. However, Jayawardena et al. (2016a) included more strains of $C$. truncatum and concluded that $C$. aciculare is not a new species, but $C$. truncatum itself. Therefore, $C$. aciculare was synonymised under C. truncatum (Jayawardena et al. 2016a).

It is clear that insufficient taxon sampling is one of the main reasons that lead to ambiguous species boundaries in Colletotrichum. Therefore, it is highly recommended to include multiple strains from diverse origins for delimiting species or introducing novel species in Colletotrichum.

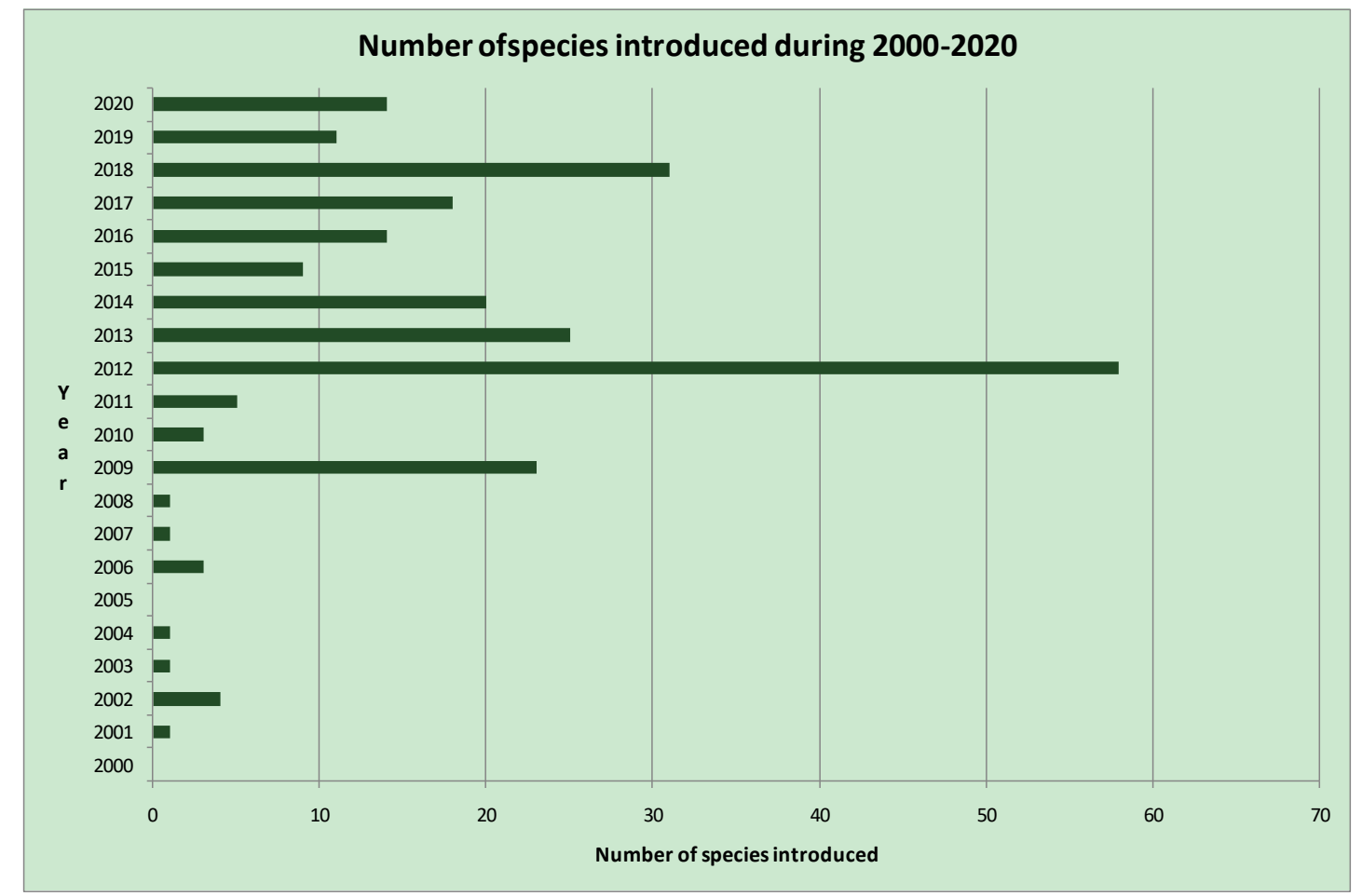

Figure 4 - Number of Colletotrichum species introduced from 2000-2020 (based on Index Fungorum)

\section{Misidentification and mistakes}

Colletotrichum is mainly identified based on multi-locus phylogenetic analyses. However, errors or mistakes in the sequence data can result in erroneous new species that are not present in nature. Ariyawansa et al. (2015) introduced Colletotrichum hymenocallidicola, a singleton species based on one strain using five loci (ITS, gapdh, chs-1, act and tub2). Damm et al. (2019) noted that the placement of the species based on $a c t$ and $t u b 2$ is different from the placement based on ITS, gapdh and chs- 1 sequence data using a BLASTn search. Closest matches of the ITS, gapdh and chs-1 sequence data were $98-99 \%$ identical to strains identified as $C$. cliviae (in the orchidearum complex), while the act sequence was $93 \%$ identical with those of $C$. pseudomajus and C. vietnamense (in the gigasporum complex) and the tub2 sequence was $100 \%$ identical with the ex-epitype strain of $C$. truncatum. There is a possibility that both act and tub2 sequence data were mixed-up with those of other strains studied by the authors. Damm et al. (2019) also found that the first 22bp of gapdh sequence includes artefacts as it is completely different from all related species. We strongly recommend mycologists to perform a blast search of each gene region as well as to check the quality of sequences before conducting analyses. 
During the past five years, a significant mistake is the exclusion of information of the holotype details in the publication. Colletotrichum corchorum-capsularis a pathogen on Corchorus capsularis in China was introduced in the truncatum complex (Niu et al. 2016). However, as the authors have not listed an authentic dried type specimen, this species is invalid. A living culture was proposed as a holotype instead of a herbarium material or an inactive material. Colletotrichum australisinense, $C$. bannanense, $C$. ledongense and $C$. sichuanensis are additional examples of the same mistake (Liu et al. 2016b, 2018). We recommend that authors should be more careful when defining the material examined and review the articles before publication.

\section{Species complexes}

There are 14 species complexes accepted in Colletotrichum. For each of the complex, accepted species, host and geographical distribution (will consider only the ones that have molecular data), information of the type/reference, importance gene in identifying and uses when available are given. The type species included in Jayawardena et al. (2020) are used as the starting point for this study. All the species that have been published until June 2020 are included in the analyses (some of the dubious ones are not included). Species of each complex are listed in alphabetical order, hosts and distributions are listed chronologically.

\section{Acutatum species complex}

This species complex consists of 39 species that include $C$. acutatum and its close relatives. Members of this species complex often cause fruit rots (Damm et al. 2012b). Most species within this complex have conidia with at least one acute end (Damm et al. 2012b). A combined gene analysis for this complex using ITS, gapdh, chs-1, act and tub2 sequences data is given in Fig. 5, Table 4. Species are in alphabetical order, hosts and distributions are listed chronologically. In order to differentiate species within this complex effectively, the use of both tub2 and gapdh are recommended (Damm et al. 2012b).

Colletotrichum abscissum Pinho \& O.L. Pereira, Persoonia 34: 237 (2015)

Sexual morph not reported. See Crous et al. (2015) for illustrations and descriptions of asexual morph.

Type - Brazil, São Paulo, Brotas, on flowers of Citrus sinensis var. Valencia (sweet orange) with petal necrosis, 12 July 2014, A.L. Silveira, holotype VIC 42850, culture ex-type COAD 1877

Hosts/substrates - Citrus sinensis var. Valencia (Crous et al. 2015), Psidium guajava (Braganca et al. 2016).

Distribution - Brazil, USA (Crous et al. 2015, Braganca et al. 2016)

Notes - Colletotrichum abscissum is phylogenetically close but clearly distinct from C. costaricense by its shorter and narrower conidia. This species was identified associated with Postbloom fruit drop of Citrus and the pathogenicity based on Kochs' postulates has not been confirmed. Whole-genome data for this species are available (https://mycocosm.jgi.doe.gov/Colletotrichum/Colletotrichum.info.html).

Colletotrichum arboricola M. Zapata, M.A. Palma \& Piont., in Crous et al., Persoonia 41: 353 (2018)

See Crous et al. (2018a) for illustrations and descriptions of asexual and sexual morphs.

Type - Chile, Los Ángeles, on leaves of Fuchsia magellanica, 11 July 2012, J. Jure, holotype RGM 2481, culture ex-type CBS 144795.

Hosts/substrates - Fuchsia magellanica (Crous et al. 2018a)

Distribution - Chile (Crous et al. 2018a)

Notes - Colletotrichum arboricola is separated from other species by gapdh and act sequence data, with gapdh as the best (Crous et al. 2018a). This species is presently recorded from Fuchsia magellanica from Chile. Further collections are required to determine the host range of this species. 


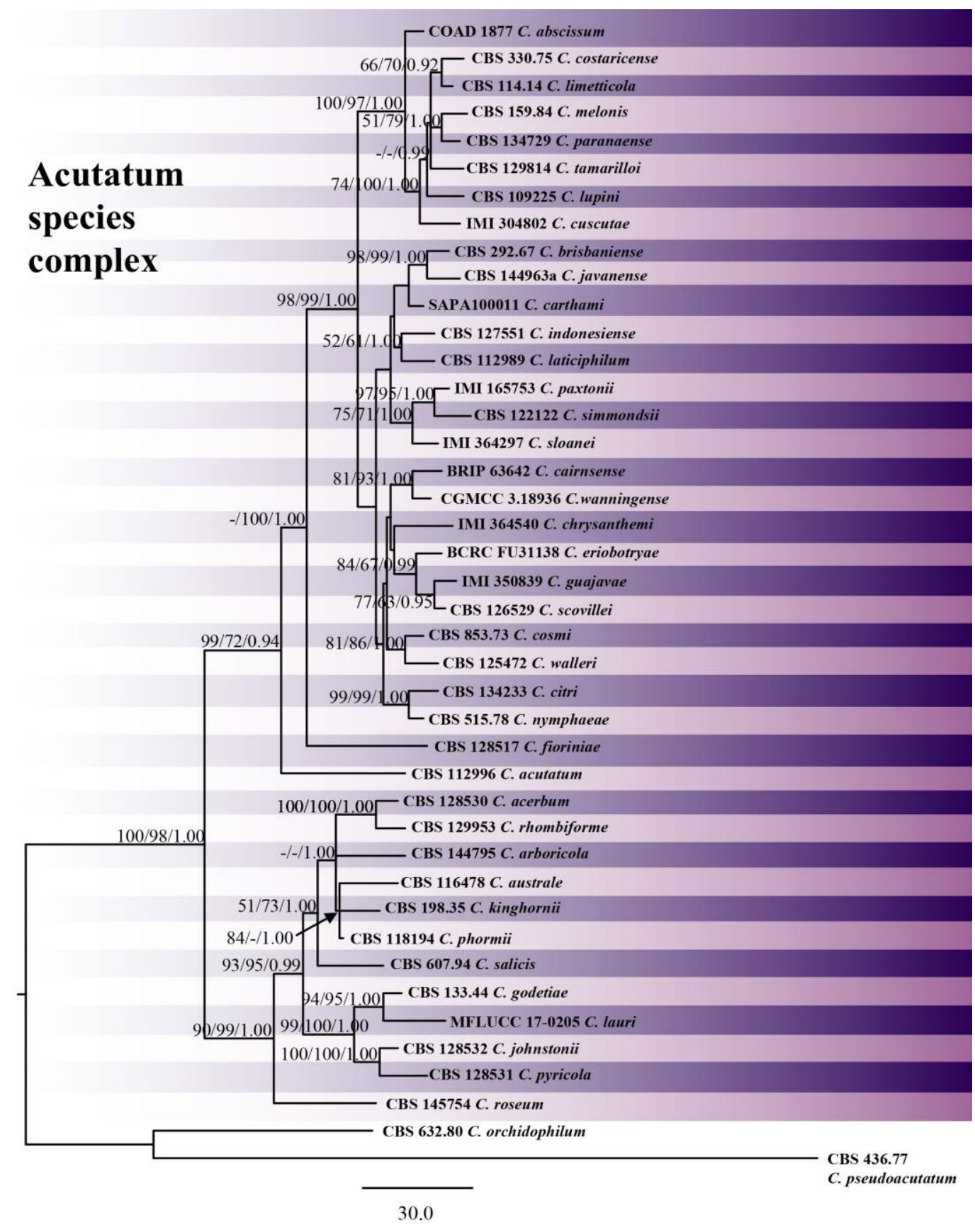

Figure 5 - The acutatum complex. One of the 10 most parsimonious trees obtained from a heuristic search of combined ITS, gapdh, chs-1, act and tub2 sequence data. The MP and ML bootstrap support values $\geq 50 \%$ and BYPP $\geq 0.90$ are indicated at the nodes and branches. The tree is rooted with $C$. roseum (CBS 145754) and C. pseudoacutatum (CBS 436.77).

Colletotrichum acerbum Damm, P.F. Cannon \& Crous, in Damm et al., Stud. Mycol. 73: 43 (2012)

Sexual morph not reported. See Damm et al. (2012b) for illustrations and descriptions of asexual morph. Type - New Zealand, Nelson, from bitter rot on fruit of Malus domestica,1 Aug. 1987, P.R. Johnston, holotype CBS H-20725, culture ex-type CBS 128530

Hosts/substrates - Malus domestica (Damm et al. 2012b)

Distribution - New Zealand (Damm et al. 2012b)

Notes - Colletotrichum acerbum is phylogenetically closer to C. rhombiforme and can be distinguished from ITS and tub2 sequence data. Conidia of $C$. acerbum are longer and the appressoria are shorter and wider than C. rhombiforme (Damm et al. 2012b). This species was 
isolated from bitter rot of apple. However, the pathogenicity of this species on apples is not confirmed via Kochs' postulates

Table 4 GenBank accession numbers of species in the acutatum complex

\begin{tabular}{|c|c|c|c|c|c|c|c|}
\hline Species name & Type/Reference strain & ITS & gapdh & chs -1 & act & tub2 & References \\
\hline C. abscissum & COAD 1877 & KP843126 & KP843129 & KP843132 & KP843141 & KP843135 & Crous et al. (2015) \\
\hline C. acerbum & CBS 128530 & JQ948459 & JQ948790 & JQ949120 & JQ949780 & JQ950110 & Damm et al. (2012b) \\
\hline C. acutatum & CBS 112996 & JQ005776 & JQ948677 & JQ005797 & JQ005839 & JQ005860 & Damm et al. (2012b) \\
\hline C. arboricola & CBS 144795 & MH817944 & MH817950 & & MH817956 & MH817962 & Crous et al. (2018) \\
\hline C. australe & CBS 116478 & JQ948455 & JQ948786 & JQ949116 & JQ949776 & JQ950106 & Damm et al. (2012b) \\
\hline C. brisbanense & CBS 292.67 & JQ948291 & JQ948621 & JQ948952 & JQ949612 & JQ949942 & Damm et al. (2012b) \\
\hline C. cairnsense & BRIP 63642 & KU923672 & KU923704 & KU923710 & KU923716 & KU923688 & de Silva et al. (2017) \\
\hline C. carthami & SAPA100011 & AB696998 & & & & AB696992 & Damm et al. (2012b) \\
\hline C. chrysanthemi & IMI 364540 & JQ948273 & JQ948603 & JQ948934 & JQ949594 & JQ949924 & Damm et al. (2012b) \\
\hline C. citri & CBS 134233 & KC293581 & KC293741 & KY856138 & KY855973 & KC293661 & Crous et al. (2015) \\
\hline C. cosmi & CBS 853.73 & JQ948274 & JQ948604 & JQ948935 & JQ949595 & JQ949925 & Damm et al. (2012b) \\
\hline C. costaricense & CBS 330.75 & JQ948180 & JQ948510 & JQ948841 & JQ949501 & JQ949831 & Damm et al. (2012b) \\
\hline C. cuscutae & IMI 304802 & JQ948195 & JQ948525 & JQ948856 & JQ949516 & JQ949846 & Damm et al. (2012b) \\
\hline C. fioriniae & CBS 128517 & JQ948292 & JQ948622 & JQ948953 & JQ949613 & JQ949943 & Damm et al. (2012b) \\
\hline C. godetiae & CBS 133.44 & JQ948402 & JQ948733 & JQ949063 & JQ949723 & JQ950053 & Damm et al. (2012b) \\
\hline C. guajavae & IMI 350839 & JQ948270 & JQ948600 & JQ948931 & JQ949591 & JQ949921 & Damm et al. (2012b) \\
\hline C. indonesiense & CBS 127551 & JQ948288 & JQ948618 & JQ948949 & JQ949609 & JQ949939 & Damm et al. (2012b) \\
\hline C. javanense & CBS 144963a & MH846576 & MH846572 & MH846573 & MH846575 & MH846574 & de Silva et al. (2019) \\
\hline C. johnstonii & CBS 128532 & JQ948444 & JQ948775 & JQ949105 & JQ949765 & JQ950095 & Damm et al. (2012b) \\
\hline C. kinghornii & CBS 198.35 & JQ948454 & JQ948785 & JQ949115 & JQ949775 & JQ950105 & Damm et al. (2012b) \\
\hline C. laticiphilum & CBS 112989 & JQ948289 & JQ948619 & JQ948950 & JQ949610 & JQ949940 & Damm et al. (2012b) \\
\hline C. lauri & MFLUCC 17-0205 & KY514347 & KY514344 & KY514341 & KY514338 & KY514350 & Hyde et al. (2017) \\
\hline C. limetticola & CBS 114.14 & JQ948193 & JQ948523 & JQ948854 & JQ949514 & JQ949844 & Damm et al. (2012b) \\
\hline C. lupine & CBS 109225 & JQ948155 & JQ948485 & JQ948816 & JQ949476 & JQ949806 & Damm et al. (2012b) \\
\hline C. melonis & CBS 159.84 & JQ948194 & JQ948524 & JQ948855 & JQ949515 & JQ949845 & Damm et al. (2012b) \\
\hline C. nymphaeae & CBS 515.78 & JQ948197 & JQ948527 & JQ948858 & JQ949518 & JQ949848 & Damm et al. (2012b) \\
\hline C. paranaense & CBS 134729 & KC204992 & KC205026 & KC205043 & KC205077 & KC205060 & Bragança et al. (2016) \\
\hline C. paxtonii & IMI 165753 & JQ948285 & JQ948615 & JQ948946 & JQ949606 & JQ949936 & Damm et al. (2012b) \\
\hline C. phormii & CBS 118194 & JQ948446 & JQ948777 & JQ949107 & JQ949767 & JQ950097 & Farr et al. (2006) \\
\hline C. pyricola & CBS 128531 & JQ948445 & JQ948776 & JQ949106 & JQ949766 & JQ950096 & Damm et al. (2012b) \\
\hline C. rhombiforme & CBS 129953 & JQ948457 & JQ948788 & JQ949118 & JQ949778 & JQ950108 & Damm et al. (2012b) \\
\hline C. roseum & CBS 145754 & MK903611 & MK903603 & & MK903604 & MK903607 & Crous et al. (2019) \\
\hline C. salicis & CBS 607.94 & JQ948460 & JQ948791 & JQ949121 & JQ949781 & JQ950111 & Damm et al. (2012b) \\
\hline C. scovillei & CBS 126529 & JQ948267 & JQ948597 & JQ948928 & JQ949588 & JQ949918 & Damm et al. (2012b) \\
\hline C. simmondsii & CBS 122122 & JQ948276 & JQ948606 & JQ948937 & JQ949597 & JQ949927 & Damm et al. (2012b) \\
\hline
\end{tabular}


Table 4 Continued.

\begin{tabular}{|c|c|c|c|c|c|c|c|}
\hline Species name & Type/Reference strain & ITS & gapdh & chs-1 & act & tub2 & References \\
\hline C. sloanei & IMI 364297 & JQ948287 & JQ948617 & JQ948948 & JQ949608 & JQ949938 & Damm et al. (2012b) \\
\hline C. tamarilloi & CBS 129814 & JQ948184 & JQ948514 & JQ948845 & JQ949505 & JQ949835 & Damm et al. (2012b) \\
\hline C. walleri & CBS 125472 & JQ948275 & JQ948605 & JQ948936 & JQ949596 & JQ949926 & Damm et al. (2012b) \\
\hline C.wanningense & CGMCC 3.18936 & MG830462 & MG830318 & MG830302 & MG830270 & MG830286 & Cao et al. (2019) \\
\hline
\end{tabular}

Colletotrichum acutatum J.H. Simmonds, Queensland J. agric. Anim. Sci. 25

Sexual morph not reported. See Damm et al. (2012b) for illustrations and descriptions of asexual morph.

Type - Australia, Queensland, Ormiston, Redlands Research Station, from fruit rot of Carica papaya, 1 Oct. 1965, J.H. Simmonds, holotypeIMI 117617

Epitype - Australia, Queensland, Brisbane, Ormiston, from fruit rot of Carica papaya, 5 Jul. 1965, J.H. Simmonds, epitype- CBS-H 20723, culture ex-epitype CBS 112996

Hosts/substrates - Anemone sp. (leaf lesion/stem spot/discoloration), Boronia megastigma, Fragaria x ananassa (fruit rot, Mangifera indica (fruit rot), Olea europaea (fruit spot), Pistacia vera, Ranunculus (seedling collapse) (Shivas \& Tan 2009, Damm et al. 2012b), Grevillea sp. (Shivas et al. 2016, Damm et al. 2012b), Aspalathus linearis (anthracnose on stems and leaves), Water used to irrigate Aspalathus linearis seedlings in a nursery, Capsicum annuum (fruit), Carica papaya, Coffea arabica (berry lesion), Hakea sericea, Hoodia sp., Leucadendron sp., Leucospermum sp., Lobelia sp. (leaf spots), Lupinus angustifolius, Mimetes sp., Nerium oleander, Olea europaea, Phlox sp. (leaf spots), Pinus radiata, Pistacia vera, Protea cynaroides, Pyrus pyrifolia, Statice sp. (Damm et al. 2012b)

Distribution - Australia (Damm et al. 2012b, Shivas \& Tan 2009, Shivas et al. 2016), Netherlands (Than et al. 2008, Damm et al. 2012b), Kenya, New Zealand, Portugal, South Africa, Sri Lanka, Tanzania, UK (Damm et al. 2012b).

Notes - Colletotrichum acutatum was first described from Redlands Horticultural Research Station, Cleveland, Queensland on papaya (Carica papaya) by Simmonds $(1965,1968)$. This species has been recognised as an important pathogen that causes anthracnose on a range of plants worldwide. Damm et al. (2012b), who designated an epitype ultimately provided taxonomic stability for this species, summarized the taxonomic history of $C$. acutatum. There are many records of $C$. acutatum identified based on morphological and cultural characteristics as well as based only on ITS sequence data in Farr \& Rossman (2021). However, many of these records require verification. Colletotrichum acutatum can be distinguished from other species in the acutatum species complex by any of the six genes analysed in Damm et al. (2012b). Pathogenicity and virulence studies for this species are available. Whole-genome sequence and genome annotation for $C$. acutatum is available (Han et al. 2016).

Colletotrichum australe Damm, P.F. Cannon \& Crous, in Damm et al., Stud. Mycol. 73 : 57 (2012)

Sexual morph not reported. See Damm et al. (2012b) for illustrations and descriptions of asexual morph.

Type - South Africa, Stellenbosch, university campus, from Trachycarpus fortunei, 2 Jan. 1998, J.E. Taylor, holotype CBS-H 20721, culture extype CBS 116478. 
Hosts/substrates - Hakea sp., Trachycarpus fortunei (Damm et al. 2012b)

Distribution - Australia, South Africa (Damm et al. 2012b)

Notes - In this species, setae are better developed and conidia are larger than in most other species in the acutatum species complex. Colletotrichum australe is distinguishable by either gapdh, his3, ITS or tub2 sequence data (Damm et al. 2012b).

Colletotrichum brisbanense Damm, P.F. Cannon \& Crous, in Damm et al., Stud. Mycol. 73: 59 (2012)

Sexual morph not reported. See Damm et al. (2012b) for illustrations and descriptions of asexual morph.

Type - Australia, Queensland, Brisbane, Eight Mile Plains, from fruit rot of Capsicum апnиит, 14 Jul. 1955, J.H. Simmonds, holotype IMI 117622, culture ex-type CBS 292.67

Hosts/substrates - Capsicum annuum (Damm et al. 2012b)

Distribution - Australia (Damm et al. 2012b)

Notes - Colletotrichum brisbanense is only recorded from the type specimen. This species was described from one of Simmonds' (1968) paratype specimens of $C$. acutatum, which was assigned to $C$. simmondsii by Shivas \& Tan (2009). The gapdh and tub2 sequences clearly separate C. brisbanense and C. simmondsii (Damm et al. 2012b). This species was isolated from fruit rot of chilli. However, the pathogenicity of this species on chilli is not confirmed via Kochs' postulates.

Colletotrichum cairnsense D.D. de Silva, R.G. Shivas \& P.W.J. Taylor, in de Silva et al., Pl. Path. 66(2): 264 (2016)

Sexual morph not reported. See de Silva et al. (2017) for illustrations and descriptions of asexual morph.

Type - Australia, Queensland, Cairns, on fruit of Capsicum annuum, 7 June 2015, R. G. Shivas, holotype BRIP 63642, culture ex-type CBS 140847.

Hosts/substrates - Capsicum annuum (de Silva et al. 2017)

Distribution - Australia (de Silva et al. 2017)

Notes - This species was isolated from infected chili fruits from Australia. Pathogenicity studies are available for this species (de Silva et al. 2017). Colletotrichum cairnsense is only recorded from Capsicum annuum and from Australia. More collections are needed to conclude the host-specificity of this species.

Colletotrichum carthami (Fukui) S. Uematsu, Kageyama, Moriwaki \& Toy. Sato, J. Gen. Pl. Path. 78(5): 326 (2012)

Epitype - Japan, Hokkaido, Sapporo, on leaves of Carthamus tinctorius, Takeo Hemmi, July 1915, epitype SAPA100011.

Hosts/substrates - Carthamus tinctorius (Uematsu et al. 2012), Calendula officinalis (Baroncelli et al. 2015a)

Distribution - Japan (Uematsu et al. 2012, Baroncelli et al. 2015a)

Notes - This species is recorded as a pathogen on Carthamus tinctorius, confirmed with pathogenicity studies (Uematsu et al. 2012, Baroncelli et al. 2015a). Colletotrichum carthami is presently recorded only from Japan.

Colletotrichum chrysanthemi (Hori) Sawada, Rep. Govt Res. Inst. Dep. Agric., Formosa 85: 81 (1943)

Sexual morph not reported. See Damm et al. (2012b) for illustrations and descriptions of asexual morph.

Reference specimen - Netherlands, Emmeloord, from twisted stem of Carthamus sp., unknown collection date and collector, culture CBS 126518

Hosts/substrates - Carthamus sp. (twisted stem), Chrysanthemum coronarium (vascular discoloration) (Damm et al. 2012b), Chrysanthemum tinctorius (Sato \& Moriwaki 2013), 
Carthamus tinctorius (Baroncelli et al. 2015a), Glebionis carinata, Glebionis coronaria (Damm et al. 2012b).

Distribution - China (Damm et al. 2012b), Czech Republic, Italy (Baroncelli et al. 2015a), Japan (Sato \& Moriwaki 2013), Netherlands (Damm et al. 2012b).

Notes - Colletotrichum chrysanthemi is separated from other species in the acutatum complex with tub2, gapdh and his3 sequence data, and its very short acute-ended conidia (Damm et al. 2012b).

Colletotrichum citri F. Huang, L. Cai, K.D. Hyde \& Hong Y. Li, in Huang et al., Fungal Diversity 61(1): 69 (2013)

Sexual morph not reported. See Huang et al. (2013) for illustrations and descriptions of asexual morph.

Type - China, Yunnan province, Ruili, on Anthracnose of a shoot of Citrus aurantifolia, August 2008, G. Q. Chen, holotype ZJUC41H, culture ex-type, CBS 134233

Hosts/substrates - Citrus aurantifolia (shoot anthracnose) (Huang et al. 2013), Vitis vinifera (ripe rot) (Lei et al. 2016), Hevea brasiliensis (leaf spot) (Hunupolagama et al. 2017)

Distribution - China (Huang et al. 2013, Lei et al. 2016), Sri Lanka (Hunupolagama et al. 2017)

Notes - This species can be differentiated from $C$. nymphaeae and $C$. limetticola by conidia shape and size. Pathogenicity studies for $C$. citri are available.

Colletotrichum cosmi Damm, P.F. Cannon \& Crous, in Damm et al., Stud. Mycol. 73: 61 (2012)

Sexual morph not reported. See Damm et al. (2012b) for illustrations and descriptions of asexual morph.

Type - Netherlands, Wageningen, from seed of Cosmos sp., collection date and collector unknown, holotype CBS H-20794, culture ex-type CBS 853.73.

Hosts/substrates - Cosmos sp. (Damm et al. 2012b)

Distribution - Netherlands (Damm et al. 2012b)

Notes - This species can be separated from other species by all gene sequences, but mostly with only 1 bp divergence. However, gapdh and his3 have more sequence divergences (Damm et al. $2012 b$ ). There is no pathogenicity or virulent studies associated with this species.

Colletotrichum costaricense Damm, P.F. Cannon \& Crous, in Damm et al., Stud. Mycol. 73: 63 (2012)

Sexual morph not reported. See Damm et al. (2012b) for illustrations and descriptions of asexual morph.

Type - Costa Rica, Meseta Central, from berry of Coffea arabica cv. 'Typica', collection date and collector unknown, holotype CBS H-20811, culture ex-type CBS 330.75.

Hosts/substrates - Coffea sp. (Damm et al. 2012b)

Distribution - Costa Rica (Damm et al. 2012b)

Notes - This species is recorded only from Coffea sp. from Costa Rica. Colletotrichum costaricense can be differentiated from the other species in the acutatum species complex by tub2, gapdh and act sequence data, most effectively with tub2 (Damm et al. 2012b). There is no pathogenicity or virulent studies related to this species. Whole genomic data are available for this species (https://mycocosm.jgi.doe.gov/Colletotrichum/Colletotrichum.info.html).

Colletotrichum cuscutae Damm, P.F. Cannon \& Crous, in Damm et al., Stud. Mycol. 73: 64 (2012)

Sexual morph not reported. See Damm et al. (2012b) for illustrations and descriptions of asexual morph.

Type - Dominica, Castle Comfort, from Cuscuta sp., 1986, C. Prior, holotype IMI 304802, culture ex-type IMI 304802. 
Hosts/substrates - Cuscuta sp. (Damm et al. 2012b)

Distribution - Dominican Republic (Damm et al. 2012b)

Notes - This species is recorded only from the type specimen. Colletotrichum cuscutae can be differentiated from $C$. lupini by longer conidia and narrower appressoria (length/width ratio). It can be separated from the other species in the acutatum species complex by tub2 and act sequence data (Damm et al. 2012b). There is no pathogenicity or virulent studies related to this species. Whole-genome data are available for this species (https://mycocosm.jgi.doe.gov/Colletotrichum/Colletotrichum.info.html).

Colletotrichum eriobotryae Damm \& C.J. Huang, in Damm, Sun \& Huang, Mycol. Progr. 19(4): 373 (2020)

Sexual morph not reported. See Damm et al. (2020) for illustrations and descriptions of asexual morph.

Type - China, Taiwan, Taichung, Xinshe District, orchard, from fruit anthracnose of Eriobotrya japonica, 14 Feb. 2017, C.J. Huang, holotype GLM-F117723, culture ex-holotype BCRC FU31138.

Hosts/substrates - Eriobotrya japonica (Damm et al. 2020)

Distribution - Taiwan, China (Damm et al. 2020)

Notes - Colletotrichum eriobotryae can be identified by its unique gapdh and act sequence data, best by gapdh (Damm et al. 2020). Pathogenicity study for this species is available and has a higher sensitivity to azoxystrobin.

Colletotrichum fioriniae (Marcelino \& Gouli) Pennycook, Mycotaxon 132(1): 150 (2017) [2016]

Sexual morph not reported. See Damm et al. (2012b) for illustrations and descriptions of asexual morph.

Type - USA, New York, Ward Pound Ridge Reserve, on mummified adult Fiorinia externa (elongate hemlock scale, insect), 2005, J.A.P. Marcelino and S. Gouli, culture ex-type CBS 128517.

Hosts/substrates - Magnifera indica (stem endophyte), Malus domestica (fruit lesion), Malus pumila, Myriophyllum spicatum (submerged stem), Olea europaea, Parthenocissus sp., cv. 'Disci' (soft rot), Penstemon sp., Persea americana (fruit rot), Piper nigrum, Primula sp. (leaf spots), Pyrus sp. (fruit rot), Rhododendron sp., Rubus sp., Solanum lycopersicum (fruit rot), Tulipa sp., Vaccinium corymbosum (fruit rot), Vitis vinifera (Damm et al. 2012b), Lycium barbarum (Liu et al. 2016b), Lycium chinense (Oo et al. 2016), Ailanthus altissima (Hyde et al. 2017), Pistacia vera (Lichtemberg et al. 2017), Litchi chinensis (Ling et al. 2020), Prunus persica (Lee et al. 2018, 2020), Solanum melongena (Xu et al. 2018b), Zanthoxylum armatum (Han et al. 2020).

Distribution - Australia, Netherlands, New Zealand, Portugal, UK, USA (Damm et al. 2012b), China (Liu et al. 2016b, Han et al. 2020, Ling et al. 2020), Korea (Oo et al. 2016, Lee et al. 2018, 2020), Italy (Hyde et al. 2017), Poland (Pszczolkowska et al. 2016).

Notes - This species was originally described as an entamopathogen from a scale insect in the USA and also been reported as an endophyte in several plants (Marcelino et al. 2008). Colletotrichum fioriniae has been reported as the causal agent of leaf and stem blight on Acacia acuminata (Golzar 2009 as C. acutatum) and fruit rot of avocado (Shivas \& Tan 2009) in Australia. Colletotrichum fioriniae is readily identified by any of the six genes analyzed by Damm et al. (2012b). There is pathogenicity or virulent studies related to this species. Whole-genome sequences data for this species is available (Barnocelli et al. 2014b, 2016).

Colletotrichum godetiae Neerg., Friesia 4(1-2): 72 (1950) [1949-50]

Sexual morph not reported. See Damm et al. (2012b) for illustrations and descriptions of asexual morph.

Type - Denmark, from seed of Clarkia hybrida cv. 'Kelvedon Glory' $463 \mathrm{C}$ in seed disinfection experiment, 17 Jun. 1943, P. Neergaard, culture ex-type CBS 133.44. 
Hosts/substrates - Aeschynomene virginica, Agrimonia eupatoria (leaf spot), Bonzai (sunken brown spots on fruit), Ceanothus sp., Citrus aurantium (fruit rot), Clarkia hybrida cv. 'Kelvon glory' (seed), Fragaria $\times$ ananassa, Fragaria vesca, Juglans regia, Laurus nobilis, Mahonia aquifolium (leaf spots), Malus sylvestris, Nut shell, Olea europaea, Parthenocissus sp. (leaf and stem spots), Podocarpus sp., Prunus sp., Rubus idaeus, Sambucus nigra, Vitis sp., Schinus molle, Solanum betaceum (fruit anthracnose), Ugni molinae (twig, tip necrosis), Acer cappadocicum, Frangula alnus (Alizadeh et al. 2015), Helleborus sp. (leaf spot), Malus domestica (fruit rot) (Shivas et al. 2016), Cornus mas (Tóth et al. 2017).

Distribution - Austria, Belgium, Chile, Colombia, France, Greece, Germany, Ireland, Israel, Italy, Mexico, Netherlands, Norway, Spain, South Africa, Turkey, UK, USA (Damm et al. 2012b), Iran (Alizadeh et al. 2015), Australia (Shivas et al. 2016), Hungary (Tóth et al. 2017).

Notes - Colletotrichum godetiae is recorded as a pathogen of fruits, leaves and stems. This species can be distinguished from other species in the acutatum species complex by any of the six genes analysed in Damm et al. (2012b). Pathogenicity or virulent studies are available for this species. Whole genomic data for this species are available

(https://mycocosm.jgi.doe.gov/Colletotrichum/Colletotrichum.info.html).

Colletotrichum guajavae Damm, P.F. Cannon \& Crous, in Damm et al., Stud. Mycol. 73: 69 (2012)

Sexual morph not reported. See Damm et al. (2012b) for illustrations and descriptions of asexual morph.

Type - India, Assam, Silchar, from fruit of Psidium guajava, collection date and collector unknown, holotype IMI 350839, culture ex-type IMI 350839.

Hosts/substrates - fruit of Psidium guajava (Damm et al. 2012b), Elettaria cardamomum (Chethana et al. 2016)

Distribution - India (Damm et al. 2012b, Chethana et al. 2016)

Notes - Colletotrichum guajavae cannot reliably distinguished from species in the acutatum complex using morphological characteristics. This species can be distinguished from other species using tub2, gapdh and act sequence data, most effectively with gapdh (Damm et al. 2012b). Pathogenicity or virulent studies have been carried out to prove pathogenicity of this species on Elettaria cardamomum (Chethana et al. 2016).

Colletotrichum indonesiense Damm, P.F. Cannon \& Crous, in Damm et al., Stud. Mycol. 73: 71 (2012)

Sexual morph not reported. See Damm et al. (2012b) for illustrations and descriptions of asexual morph.

Type - Indonesia: Sumatra, Tele, from leaf spots developing after herbicide treatment of Eucalyptus sp., 1 Jan. 2008, M.J. Wingfield, holotype CBS H-20798, culture ex-type CBS 127551.

Hosts/substrates - Eucalyptus sp.

Distribution - Indonesia

Notes - Colletotrichum indonesiense can be distinguished from other species in the acutatum species complex by tub2, act, gapdh and chs-1 sequence data, and most effectively with tub2 (Damm et al. 2012b). Even though this species was isolated from a leaf spot, no pathogenicity or virulence data are available.

Colletotrichum javanense D.D. de Silva, Crous \& P.W.J. Taylor, in Silva et al., IMA Fungus 10(1): 20 (2019)

Sexual morph not reported. See de Silva et al. (2019) for illustrations and descriptions of asexual morph.

Type - Indonesia: West Java, Purwakata regency, on fruit of Capsicum annuum, Dec. 2014, F. Fitriyah holotype CBS H-14496, ex-type culture CBS 144963.

Hosts/substrates - Capsicum annuиm (de Silva et al. 2019) 
Distribution - Indonesia (de Silva et al. 2019)

Notes - Colletotrichum javanense was introduced based on a single strain. This species can be distinguished by the presence of distinct chlamydospores from its sister taxon $C$. brisbanense (de Silva et al. 2019). Pathogenicity studies for this species are available. This species is presently recorded only from its type locality and host.

Colletotrichum johnstonii Damm, P.F. Cannon \& Crous, in Damm et al., Stud. Mycol. 73 : 72 (2012)

Sexual morph not reported. See Damm et al. (2012b) for illustrations and descriptions of asexual morph.

Type - New Zealand, Auckland, from fruit rot of Solanum lycopersicum, 29 Feb. 1990, J.M. Dingley, holotype CBS H-20809, culture ex-type CBS 128532.

Hosts/substrates - Citrus sp. (fruit rot), Solanum lycopersicum (fruit rot) (Damm et al. 2012b)

Distribution - New Zealand (Damm et al. 2012b)

Notes - Based on the data available $C$. johnstonii appears to be endemic to New Zealand, but is not host-specific (Damm et al. 2012b, Jayawardena et al. 2016a). This species can be separated from other species based on act, his3, tub2 and gapdh sequence data, act being the best (Damm et al. 2012b). There is no pathogenicity or virulence data available for this species.

Colletotrichum kinghornii Damm, P.F. Cannon \& Crous, in Damm et al., Stud. Mycol. 73: 73 (2012)

Sexual morph not reported. See Damm et al. (2012b) for illustrations and descriptions of asexual morph.

Type - UK, Scotland, from Phormium tenax, unknown collection date, N.L. Alcock, holotype CBS H-20909, culture ex-type CBS 198.35.

Hosts/substrates - Phormium sp. (Damm et al. 2012b)

Distribution - UK (Damm et al. 2012b)

Notes - Colletotrichum kinghornii is one of the two species in the acutatum complex with larger conidia is most effectively separated from other species using his 3 sequence data (Damm et al. 2012b).

Colletotrichum laticiphilum Damm, P.F. Cannon \& Crous, in Damm et al., Stud. Mycol. 73: 74 (2012)

Sexual morph not reported. See Damm et al. (2012b) for illustrations and descriptions of asexual morph.

Type - India, Kerala, Kottayam, Rubber Research Institute campus, from raised spots on leaf of Hevea brasiliensis, 1999, unknown collector, holotype CBS H-20799, culture ex-type CBS 112989.

Hosts/substrates - Hevea brasiliensis (Damm et al. 2012b, Shi et al. 2019)

Distribution - India, Colombia (Damm et al. 2012b), Sri Lanka (Hunupolagama et al. 2017), China (Shi et al. 2019)

Notes - Colletotrichum laticiphilum is separated from other species by its tub2, gapdh and chs- 1 sequence data, and most differentially with tub2 (Damm et al. 2012b). Pathogenicity studies have been conducted for this species to confirm its pathogenicity.

Colletotrichum lauri Jayaward., Camporesi \& K.D. Hyde, in Hyde et al., Index Fungorum 347: 1 (2017)

Sexual morph not reported. See Hyde et al. (2017) for illustrations and descriptions of asexual morph.

Type - Italy Province of Forlì-Cesena [FC], San Lorenzo in Noceto - Forlì, on dead aerial leaves of Laurus nobilis, 25 May 2015, E. Camporesi, IT 2505, holotype MFLU 15-1374, culture ex-type MFLUCC 17-0205. 
Hosts/substrates - Laurus nobilis (Hyde et al. 2017)

Distribution - Italy (Hyde et al. 2017)

Notes - Colletotrichum lauri presently is recorded only from Laurus nobilis in Italy.

Colletotrichum limetticola (R.E. Clausen) Damm, P.F. Cannon \& Crous [as 'limetticolum'], in Damm et al., Stud. Mycol. 73: 76 (2012)

Sexual morph not reported. See Damm et al. (2012b) for illustrations and descriptions of asexual morph.

Type - USA, Florida, from young twig of Citrus aurantifolia, collection date and collector unknown, epitype CBS H-20910, culture ex-epitype CBS114.14

Hosts/substrates - Citrus aurantifolia (Damm et al. 2012b), Malus domestica (leaf spot, Moreira et al. 2019), Vitis vinifera (ripe rot of grapes, Echeverrigaray et al. 2020)

Distribution - Cuba, USA (Damm et al. 2012b), Brazil (Moreira et al. 2019, Echeverrigaray et al. 2020)

Notes - Colletotrichum limetticola is distinguished from other species by tub2, gapdh and his 3 , most effectively with tub2 sequence data. Pathogenicity studies for this species are available.

Colletotrichum lupini (Bondar) Damm, P.F. Cannon \& Crous, in Damm et al., Stud. Mycol. 73: 78 (2012)

Sexual morph not reported. See Damm et al. (2012b) for illustrations and descriptions of asexual morph.

Type - Germany, from Lupinus albus, unknown date, U. Feiler, culture ex-holotype of C. lupini var. setosum, CBS 109221.

Hosts/substrates - Camellia sp., Cinnamomum verum, Lupinus albus, Lupinus angustifolius, Lupinus mutabilis, Lupinus polyphyllus, Manihot utilissima (Damm et al. 2012b)

Distribution - Austria, Australia, Bolivia, Canada, Costa Rica, France, Germany, Netherlands, Poland, Portugal, Rwanda, South Africa, UK, Ukraine, USA (Damm et al. 2012b)

Notes - Colletotrichum lupini was originally described from Lupinus albus in the São Paulo region of Brazil, most probably introduced to South America along with its host plant, which is native to the Mediterranean region (Kurlovich 2002). This species is an economically important species on Lupinus worldwide and there is a significant interest in breeding of resistant varieties (Adhikari et al. 2011). Colletotrichum lupini can be distinguished from other species in the acutatum species complex by most of the six genes (except actin) analysed by Damm et al. (2012b), with $t u b 2$ providing the best resolution. Colletotrichum lupini has been isolated from leaf spots, petiole with sunken spots (Damm et al. 2012b). Pathogenicity studies for this species have been conducted to establish the pathogenicity and virulence of the species. Whole-genome sequence is available for this species (Carbú et al. 2019). Dubrulle et al. (2020) using transcriptomic and proteomic analysis deciphered the infection process of $C$. lupini.

Colletotrichum melonis Damm, P.F. Cannon \& Crous, in Damm et al., Stud. Mycol. 73: 80 (2012)

Sexual morph not reported. See Damm et al. (2012b) for illustrations and descriptions of asexual morph.

Type - Brazil, from peel of fruit of Cucumis melo, unknown collector and collection date, holotype CBS H-20785, culture ex-type CBS 159.84

Hosts/substrates - Cucumis melo (Damm et al. 2012b), Diospyros kaki (Carraro et al. 2019), Malus doemstica (Velho et al. 2015)

Distribution - Brazil (Damm et al. 2012b, Velho et al. 2015, Carraro et al. 2019), Uruguay (Velho et al. 2015)

Notes - This species can be separated from other species by gapdh, act and his3 sequence data, with gapdh performing best as a differential gene (Damm et al. 2012b). Pathogenicity studies are available for this species. Whole-genome sequence data of $C$. melonis is available (Grigoriev et al. 2011) https://mycocosm.jgi.doe.gov/Colletotrichum/Colletotrichum.info.html). 
Colletotrichum nymphaeae (Pass.) Aa, Netherlands Journal of Plant Pathology, Supplement 1 84(3): 110 (1978)

Sexual morph not reported. See Damm et al. (2012b) for illustrations and descriptions of asexual morph.

Type - Netherlands, Oude Waal near Nijmegen, Gem. Ubbergen, from leaf spots of Nymphaea alba, 7 Aug. 1978, G. van der Velde, epitype CBS H-20787, culture ex-epitype CBS 515.78 .

Hosts/substrates - Anemone sp. Capsicum sp., Fragaria $\times$ ananassa (fruit and crown rot, rrot discolouration, seeds), Fragaria vesca, Leucaena sp., Litter, Mahonia aquifolium, Malus pumila, Nuphar luteum (leaf spot), Nymphaea alba (leaf spot), Oenothera sp. (black staining of stem), Olea europaea, Pelargonium graveolens, Photinia sp., Protea sp., Phaseolus sp. (Damm et al.2012b), Capsicum sp. (Nasheshi et al. 2016), Robinia pseudoacacia (Yamagishi 2017), Vitis sp. (Liu et al. 2016d, Hevea brasiliensis (Hunupolagama et al. 2017), Actinidia arguta (Kim et al. 2018), Carya illinoinensis (Poletto et al. 2019, Zhang et al. 2019), Diospyros kaki (Hassan et al. 2019), Eriobotrya japonica (Wu et al. 2018, Damm et al. 2020), Ilex serrata-verticillata (Lin et al. 2018), Juglans regia (Da-Lio et al. 2018, Savian et al. 2019), Punica granatum (Xavier et al. 2019), Pyrus pyrifolia (Moreira et al. 2019), Solanum lycopersicum var. cerasiforme (Chechi et al. 2019), Camellia oliferae (Li \& Li 2020)

Distribution - Australia, Brazil, Bulgaria, Canada, France, India, Indonesia, Israel, Italy, Kenya, Mexico, Netherlands, Portugal, South Africa, Spain, Switzerland, Thailand, UK, USA, Zimbabwe (Damm et al. 2012b), Kenya (Barnocelli et al. 2015), China (Jayawardena et al. 2016b, Wu et al. 2018, Zhang et al. 2019, Damm et al. 2020, Li \& Li 2020), Malaysia (Nasehi et al. 2016), Sri Lanka (Hunupolagama et al. 2017), South Korea (Kim et al. 2018, Hassan et al. 2019)

Notes - Colletotrichum nymphaeae is well separated from other species in the acutatum species complex with tub2 sequence data (Damm et al. 2012b). Whole-genome sequence data of C. nymphaeae is available (Baroncelli et al. 2016). Pathogenicity studies have been conducted on this species to confirm its pathogenicity.

Colletotrichum paranaense C.A.D. Bragança \& Damm, in Bragança et al., Fungal Biology 120(4): 555 (2016)

Sexual morph not reported. See Braganca et al. (2016) for illustrations and descriptions of asexual morph.

Type - Brazil, Parana, from fruit anthracnose of Malus domestica, 2010, L. Mio holotype CBS H-21122, culture ex-holotype CBS 134729.

Hosts/substrates - Caryocar brasiliense (fruit anthracnose), Malus domestica (fruit rot, leaf spot), Prunus persica (fruit anthracnose) (Braganca et al. 2016, Moreira et al. 2019), Coprosma sp., Fragaria $\times$ ananassa (Moreira et al. 2019)

Distribution - Brazil (Braganca et al. 2016, Moreira et al. 2019), Australia, New Zealand (Moreira et al. 2019)

Notes - Colletotrichum paranaense can be distinguished from other species by tub2 and his3 sequence data (Braganca et al. 2016). Pathogenicity studies for this species are available. Whole genomic data for this species are available

(https://mycocosm.jgi.doe.gov/Colletotrichum/Colletotrichum.info.html).

Colletotrichum paxtonii Damm, P.F. Cannon \& Crous, in Damm et al., Stud. Mycol. 73: 85 (2012)

Sexual morph not reported. See Damm et al. (2012b) for illustrations and descriptions of asexual morph.

Type - St. Lucia, from Musa sp., 1972, P. Griffee holotype IMI 165753, culture ex-type IMI 165753

Hosts/substrates - Musa sp. (Damm et al. 2012b)

Distribution - St. Lucia (West Indies) (Damm et al. 2012b) 
Notes - Colletotrichum paxtonii can be separated from other species by tub2 and gapdh sequence data, with $t u b 2$ as the best diagnostic gene. There are two strains of this species available and are from Musa sp. from West Indies countries (Damm et al. 2012b). This species is only recorded from Musa sp. from West Indies.

Colletotrichum phormii (Henn.) D.F. Farr \& Rossman, Mycological Research 110 (12): 1403 (2006)

See Farr et al. (2006) for illustrations and descriptions of sexual morph. See Damm et al. (2012b) for illustrations and descriptions of asexual morph.

Type - APhis3 interception Port Orlando 007160, from Phormium sp., 6 Nov. 2000, W. Sheta, epitype CBS-H 20720, culture ex-epitype CBS 118194

Hosts/substrates - Phormium sp. (Farr et al. 2006, Damm et al. 2012b, Serdani et al. 2013)

Distribution - Australia, Germany, Netherlands, New Zealand, South Africa, UK (Farr et al. 2006, Golzar \& Wang 2010, Damm et al. 2012b, Serdani et al. 2013)

Notes - Colletotrichum phormii can be separated from other species by tub2, gapdh, his 3 and act sequence data, and most effectively with his3 sequence data (Damm et al. 2012b). This species is recorded only from Phormium sp. (Farr et al. 2006). Pathogenicity studies have been conducted to establish the pathogenicity of this species. Whole-genome sequence data of $C$. phormii is available (Grigoriev et al. 2011, https://mycocosm.jgi.doe.gov/Colletotrichum/Colletotrichum.info.html).

Colletotrichum pyricola Damm, P.F. Cannon \& Crous, in Damm et al., Stud. Mycol. $73: 94$ (2012) Sexual morph not reported. See Damm et al. (2012b) for illustrations and descriptions of asexual morph.

Type - New Zealand, WO, Waikato, from fruit rot of Pyrus communis, 1 Jun. 1988, unknown collector, holotype CBS H-20810, culture ex-type CBS 128531.

Hosts/substrates - Pyrus communis (Damm et al. 2012b), Daphne odora (leaf and tip dieback) (Shivas et al. 2016)

Distribution - Australia (Shivas et al. 2016), New Zealand (Damm et al. 2012b)

Notes - This fungus was first described from fruit rot of pear (Pyrus communis) in New Zealand. Colletotrichum pyricola can be distinguished from other species in the acutatum species complex with its unique act, gapdh, chs-1 and tub2 sequence data (Damm et al. 2012b). Pathogenicity studies for this species are not available.

Colletotrichum rhombiforme Damm, P.F. Cannon \& Crous, in Damm et al., Stud. Mycol. 73: 95 (2012)

See Damm et al. (2012b) for illustrations and descriptions of asexual and sexual morph.

Type - Portugal, Mirandela, Torre de D. Chama, from anthracnose on fruit of Olea europaea, Dec. 2003, P. Talhinhas, holotype CBS H-20724, culture ex-type CBS 129953.

Hosts/substrates - Olea europaea (fruit rot), Vaccinium macrocarpon (Damm et al. 2012b), Malus domestica (bitter rot) (Wu et al. 2017, Grammen et al. 2019)

Distribution - Portugal, USA (Damm et al. 2012b), China (Wu et al. 2017), Belgium (Grammen et al. 2019)

Notes - Colletotrichum rhombiforme can be separated from other species by all loci (ITS, gapdh, tub2, act, his3) studied in Damm et al. (2012b) except the chs-1 sequence data. Pathogenicity studies for this species are available.

Colletotrichum salicis (Auersw. ex Fuckel) Damm, P.F. Cannon \& Crous, in Damm et al., Stud. Mycol. 73: 97 (2012)

See Damm et al. (2012b) for illustrations and descriptions of asexual and sexual morph.

Type - Netherlands, Z. Flevoland, Salix forest near Blocq van Kuffeler, from leaf spot of Salix sp., 11 Sep. 1994, H.A. van der Aa, epitype CBS H-20730, culture ex-epitype CBS 607.94. 
Hosts/substrates - Acer platanoides, Araucaria excelsa (anthracnose and dieback), Fragaria $\times$ ananassa (petiole spot, fruit rot), Malus domestica, Populus sp., Pyrus pyrifolia (fruit rot), Rhododendron sp., Salix sp. (leaf spot, twig lesions), Solanum lycopersicum (Damm et al. 2012b)

Distribution - Germany, Japan, Latvia, Netherlands, New Zealand, Sweden, UK, USA (Damm et al. 2012b), Belgium (Grammen et al. 2018)

Notes - It is one of the few species to produce fertile ascomata in culture (Damm et al. 2012b). Colletotrichum salicis can be separated from other species by all genes (gapdh, tub2, act, chs-1, gs, his3), except for ITS (Damm et al. 2012b). Whole-genome sequence data (Baroncelli et al. 2016) and pathogenicity studies for this species are available.

Colletotrichum scovillei Damm, P.F. Cannon \& Crous, in Damm et al., Stud. Mycol. 73: 100 (2012)

Sexual morph not reported. See Damm et al. (2012b) for illustrations and descriptions of asexual morph.

Type - Indonesia, from Capsicum sp., collection date and collector unknown, holotype CBS H-20792, culture ex-type CBS 126529.

Hosts/substrates - Capsicum annuum, Capsicum frutescens (Damm et al. 2012b, Caires et al. 2014, Kanto et al. 2014, Zhao et al. 2016a, Diao et al. 2017, Noor \& Zakaria 2018), Clausena lansium (Lin et al. 2020), Mangifera indica (Qin et al. 2019), Musa sp. (Zhou et al. 2017)

Distribution - Indonesia, Thailand (Damm et al. 2012b), Brazil (Caires et al. 2014), China (Zhao et al. 2016a, Diao et al. 2017, Zhou et al. 2017, Qin et al. 2019, Lin et al. 2020), Japan (Kanto et al. 2014), Malaysia (Noor \& Zakaria 2018), South Korea (Oo et al. 2017), Taiwan (Wu et al. 2020)

Notes - Colletotrichum scovillei can be separated from other species by tub2, gapdh and act sequences data, with gapdh being the best (Damm et al. 2012b). Pathogenicity studies for this species are available.

Colletotrichum simmondsii R.G. Shivas \& Y.P. Tan, Fungal Diversity 39: 119 (2009)

Sexual morph not reported. See Damm et al. (2012b) for illustrations and descriptions of asexual morph.

Type - Australia, Queensland, Yandina, from fruit anthracnose of Carica papaya, May 1987, L.M. Coates, culture ex-holotype CBS 122122.

Hosts/substrates - Actinidia chinensis, Averrhoa carambola, Capsicum frutescens, Cyphomandra betacea, Litchi chinensis, Lycopersicon esculentum, Nephelium lappaceum, Olea sp., Persea americana, Vaccinium corymbosum, Vitis sp. (Shivas \& Tan 2009), Carica papaya, Cyclamen sp. (deformations and brown staining of stem tip), Fragaria $\times$ ananassa (fruit rot), Mangifera indica, Protea cynaroides (Damm et al. 2012b).

Distribution - Australia, Netherlands, USA (Damm et al. 2012b).

Notes - Colletotrichum simmondsii is separable from other species by gapdh and tub2 sequencing data (Damm et al. 2012b). Whole-genome sequence data for this species is available (Barnocelli et al. 2016).

Colletotrichum sloanei Damm, P.F. Cannon \& Crous, in Damm et al., Stud. Mycol. 73: 103 (2012) Sexual morph not reported. See Damm et al. (2012b) for illustrations and descriptions of asexual morph.

Type - Malaysia, Borneo, Sabah, Tuaran, from leaf of Theobroma cacao, 1994, A.R. Rossman and C.L. Bong, holotype IMI 364297, culture ex-type IMI 364297

Hosts/substrates - Litchi chinensis, Theobroma cacao (Damm et al. 2012b)

Distribution - Australia (Shivas et al. 2016), Malaysia (Damm et al. 2012b)

Notes - This species is most easily distinguished from other species in the acutatum species complex with tub2, his3 and act sequence data (Damm et al. 2012b). Little is known about C. sloanei host association and pathogenicity. 
Colletotrichum tamarilloi Damm, P.F. Cannon \& Crous, in Damm et al., Stud. Mycol. 73: 105 (2012)

Sexual morph not reported. See Damm et al. (2012b) for illustrations and descriptions of asexual morph.

Type - Colombia, Cundinamarca, from fruit anthracnose of Solanum betaceum, 13 Aug. 2010, J. Molina, holotype CBS H-20726, culture ex-type CBS 129814.

Hosts/substrates - Solanum betaceum (anthracnose) (Damm et al. 2012b), Cyphomandra betacea (Baroncelli et al. 2015b).

Distribution - Colombia (Damm et al. 2012b, Baroncelli et al. 2015b).

Notes - Colletotrichum tamarilloi can be separated from other species using chs-1, his3, tub2 and gapdh sequence data, most effectively with gapdh (Damm et al. 2012b). This species is presently only recorded in Colombia. Whole genomic data for this species are available (https://mycocosm.jgi.doe.gov/Colletotrichum/Colletotrichum.info.html).

Colletotrichum walleri Damm, P.F. Cannon \& Crous, in Damm et al., Stud. Mycol. 73: 106 (2012)

Sexual morph not reported. See Damm et al. (2012b) for illustrations and descriptions of asexual morph.

Type - Vietnam, Buon Ma Thuot-Dak Lac, from leaf tissue of Coffea arabica, unknown collection date, H. Nguyen, holotype CBS H-20795, culture extype CBS 125472

Hosts/substrates - Coffea sp. (Damm et al. 2012b)

Distribution - Vietnam (Damm et al. 2012b)

Notes - This species can be separated from other species in this complex by using any of the genes used by Damm et al. (2012b), most effectively with ITS and his3 sequence data. Colletotrichum walleri is recorded only from Coffea sp. in Vietnam.

Colletotrichum wanningense X.R. Cao, H.Y. Che \& D.Q. Luo, in Cao et al., Pl. Dis. 103(1): 120 (2018)

Sexual morph not reported. See Cao et al. (2019) for illustrations and descriptions of asexual morph.

Type - China, Hainan province, Wanning city, on a living leaf of Hevea brasiliensis, 1 April 2017, X. R. Cao, holotype HMAS 247814, culture extype CGMCC 3.18936.

Hosts/substrates - Hevea brasiliensis (Cao et al. 2019)

Distribution - China (Cao et al. 2019)

Notes - Colletotrichum wanningense is phylogenetically close to C. cairnsense (Cao et al. 2019). It is presently recorded only from Hevea brasiliensis and in China. Pathogenicity studies for C. wanningense are available.

\section{Agaves Species Complex}

This species complex is defined as a collective of $C$. agaves and four closely related species. Bhunjun et al. (2021) established this complex based on phylogenetic and evolutionary evidence. All species in this complex appear to be host specific. However, for some of these species only a single strain is available. A combined gene analysis for this complex using ITS, gapdh, chs-1, act and $t u b 2$ sequence data is given in Fig. 6, Table 5.

Colletotrichum agaves Cavara, Fung. Long. Exsicc. 3: no. 100 (1892)

Sexual morph not reported. See Farr et al. (2006) for illustrations and descriptions of asexual morph.

Type - Italy, Pavia, Ticinum, in botanical garden, on decaying leaves of cultivated Agave americana, 1904, Hedgcock G. holotype BPI 397108, culture ex-type CBS 118190.

Hosts/substrates - Agave spp. (Farr et al. 2006)

Distribution - Mexico, Netherlands (Farr et al. 2006) 
Notes - Most of the records of this species are based on morphological characteristics alone. Hence we have not included them into the hosts and distribution. Based on Farr et al. (2006) this species may have a distribution from warm temperate and tropical regions including southern Europe and the Neotropics. Pathogenicity studies for this species are not available.

Colletotrichum euphorbiae Damm \& Crous, in Crous et al., Persoonia 31: 203 (2013)

Sexual morph not reported. See Crous et al. (2013) for illustrations and descriptions of asexual morph.

Type - South Africa, Western Cape Province, Kirstenbosch Botanical Garden, on leaves of Euphorbia sp., Sept. 2012, M.J. Wingfield, holotype CBS H-21409, culture ex-type CBS 134725.

Hosts - Euphorbia sp. (Crous et al. 2013)

Distribution - South Africa (Crous et al. 2013)

Notes - This taxon can be distinguished from the other species from its longer conidia (Crous et al. 2013). Colletotrichum euphorbiae is presently recorded only from Euphorbia sp. in South Africa.

Colletotrichum ledebouriae Crous \& M.J. Wingf., in Crous et al., Persoonia 36: 331 (2016)

Sexual morph not reported. See Crous et al. (2016) for illustrations and descriptions of asexual morph.

Type - South Africa, Eastern Cape Province, Haga Haga, on leaves of Ledebouria floridunda, Dec. 2014, M.J. Wingfield, holotype CBS H-22593, culture ex-type CBS 141284.

Hosts/substrates - Ledebouria floridunda (Crous et al. 2016)

Distribution - South Africa (Crous et al. 2016)

Notes - Colletotrichum ledebouriae is only recorded from its type strain. This species was isolated from diseased leaves. However, no pathogenicity study has been carried out to confirm this.

Colletotrichum neosansevieriae Crous \& N.A. van der Merwe, Persoonia, Mol. Phyl. Evol.

Fungi 34: 221 (2015)

Sexual morph not reported. See Crous et al. (2015) for illustrations and descriptions of asexual morph.

Type - South Africa, Gauteng, Kwalata Game Ranch, on leaves of Sansevieria trifasciata, 25 Sept. 2014, P.W. Crous \& N.A.van der Merwe, holotype CBS H-22248, culture ex-type CBS 139918.

Hosts/substrates - Sansevieria trifasciata (Crous et al. 2015)

Distribution - South Africa (Crous et al. 2015)

Notes - This is similar to $C$. sansevieriae, however can be distinguished from phylogeny. Colletotrichum neosansevieriae was isolated as a pathogen on leaves of Sansevieria trifasciata. However, pathogenicity studies have not been carried out to confirm this. This species is recorded only from its type strain.

Colletotrichum sansevieriae Miho Nakam. \& Ohzono, in Nakamura et al. J. Gen. Pl. Path. 72(4): 253 (2006)

Sexual morph not reported. See Nakamura et al. (2006) for illustrations and descriptions of asexual morph.

Type - Japan, Kagoshima, Yoron Island, isolated from Sansevieria trifasciata cv. 'Laurentii', 1997, holotype (dried culture) MAFF239721.

Hosts/substrates - Sansevieria sp. (Nakamura et al. 2006, Aldaoud et al. 2011, Gautam et al. 2012, Karimi et al. 2017)

Distribution - Japan (Nakamura et al. 2006), Australia (Aldaoud et al. 2011), India (Gautam et al. 2012), Iran (Karimi et al. 2017) 
Notes - Colletotrichum sansevieriae appears to be host-specific. Pathogenicity studies for this species are available.

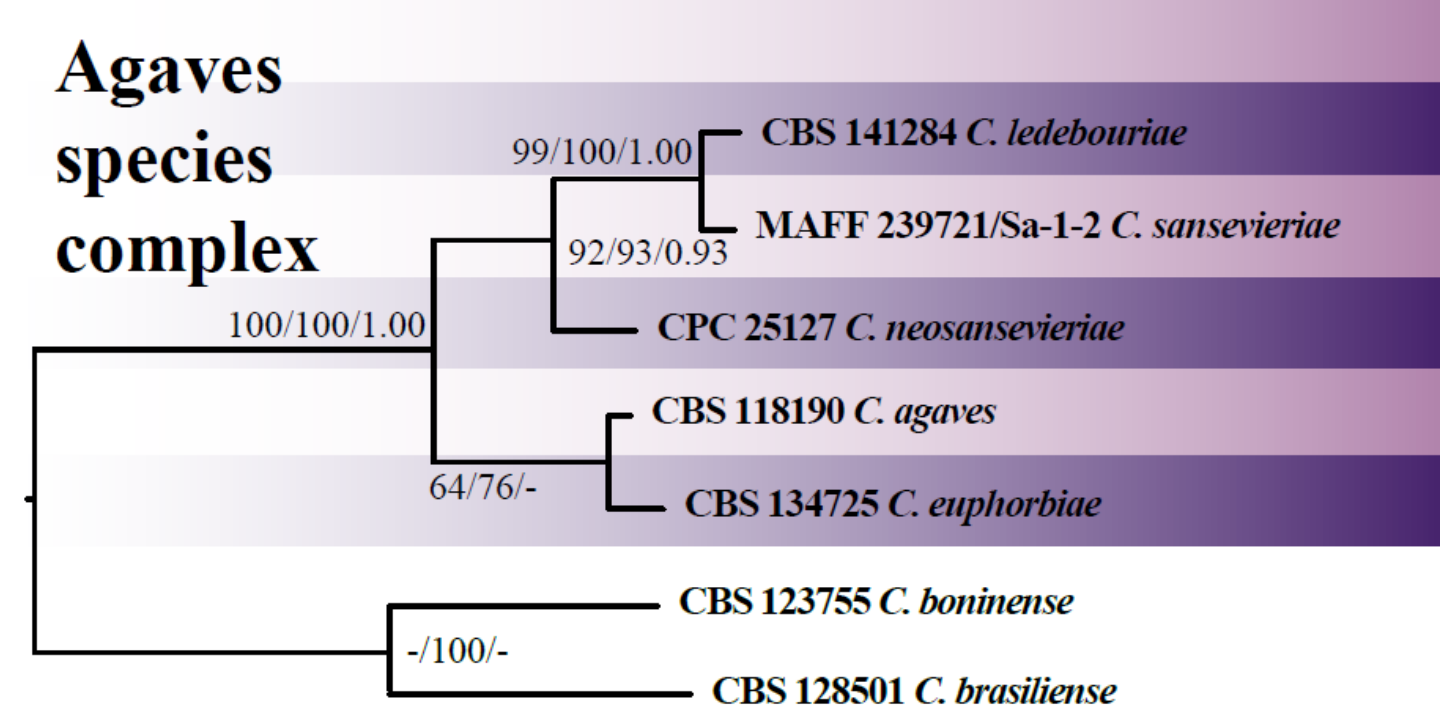

20.0

Figure 6 - The agaves complex. The most parsimonious tree obtained from a heuristic search of combined ITS, gapdh, chs-1, act and tub2 sequence data. The MP and ML bootstrap support values $\geq 50 \%$ and BYPP $\geq 0.90$ are indicated at the nodes and branches. The tree is rooted with Colletotrichum boninense (CBS 123755) and C. brasiliense (CBS 128501).

Table 5 GenBank accession numbers of the species in the agaves complex

\begin{tabular}{|c|c|c|c|c|c|c|c|}
\hline Species name & Type/Reference strain & ITS & gapdh & chs -1 & act & tub2 & References \\
\hline C. agaves & CBS 118190 & DQ286221 & & & & & Farr et al. (2006) \\
\hline C. ledebouriae & CBS 141284 & KX228254 & & & KX228357 & & Crous et al. (2016) \\
\hline C. neosansevieriae & CPC 25127 & KR476747 & KR476791 & & KR476790 & KR476797 & Crous et al. (2015) \\
\hline C. sansevieriae & MAFF 239721 & AB212991 & & & & & Nakamura et al. (2006) \\
\hline C. euphorbiae & CBS 134725 & KF777146 & KF77713 & KF777128 & KF777125 & KF777247 & Crous et al. (2013) \\
\hline
\end{tabular}




\section{Boninense Species Complex}

This species complex is defined as a collective of $C$. boninense and 25 closely related species. Typical characters of species in this complex are the conidia that have a prominent basal scar as well as the conidiogenous cells with a rather prominent periclinal thickening that sometimes extends to form a new conidiogenous locus (Damm et al. 2012a). Species of this complex are pathogens or endophytes (Damm et al. 2012a). All species within this complex can be recognized with gapdh alone (Damm et al. 2012a). Some of the species in this complex appears to be host specific or restricted to a certain geographic area. However, for some of these species only a single strain is available. Therefore, new collections are recommended to establish the host distribution of these species. A combined gene analysis for this complex using ITS, gapdh, chs-1, act and tub2 sequence data is given in Fig. 7, Table 6.

Colletotrichum annellatum Damm, P.F. Cannon \& Crous, Stud. in Damm et al., Mycol. 73: 6 (2012)

See Damm et al. (2012a) for illustrations and descriptions of asexual and sexual morphs.

Type - Colombia, Meta, Villavicencio, from a leaf of Hevea brasiliensis, 13 Aug. 2010, Olga Castro, (CBS H-20693 holotype, culture ex-type CBS 129826

Hosts - Hevea brasiliensis (Damm et al. 2012a)

Distribution - Colombia (Damm et al. 2012a)

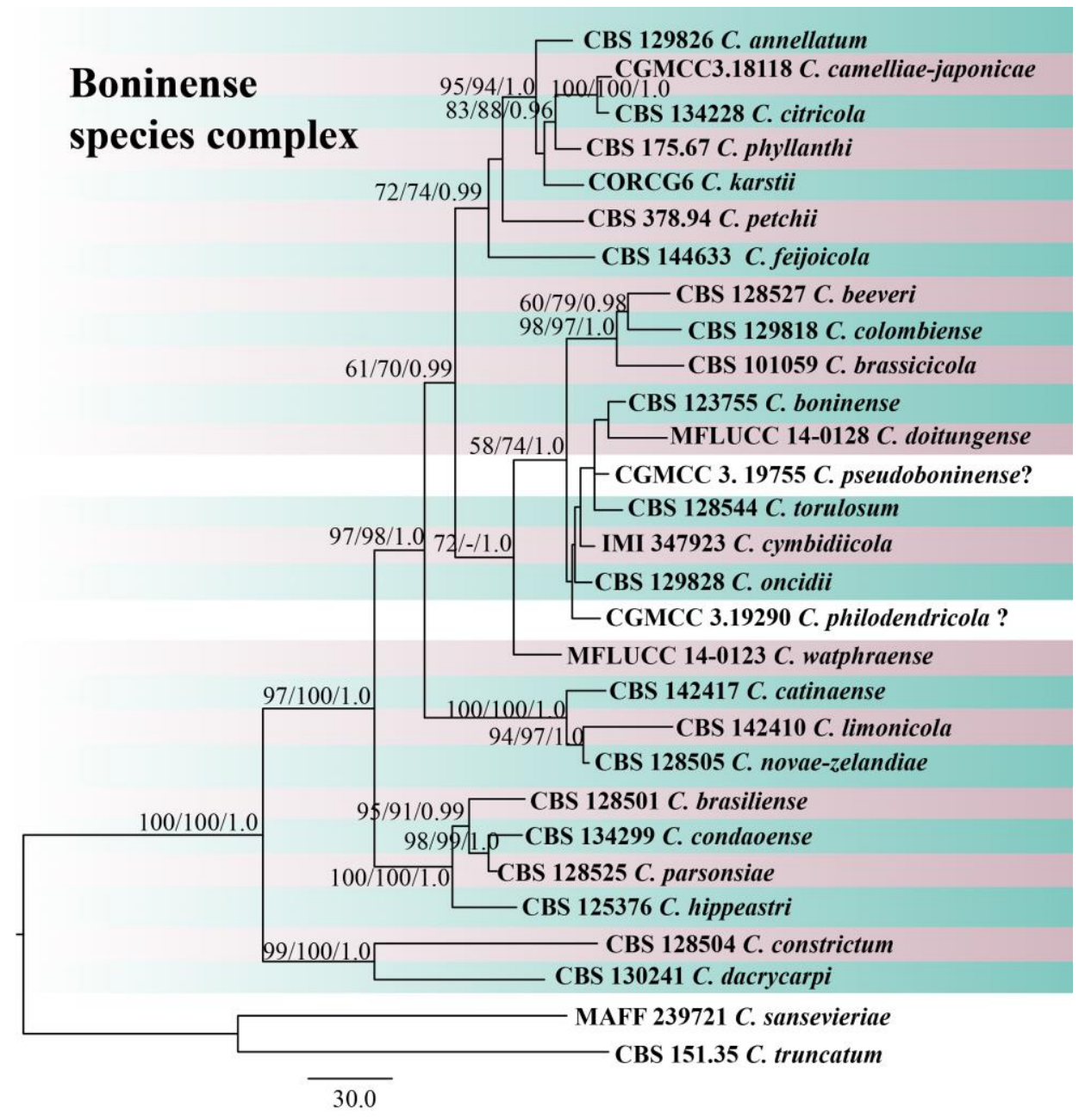

Figure 7 - The boninense complex. One of the 10 most parsimonious trees obtained from a heuristic search of combined ITS, gapdh, chs-1, act and tub2 sequence data. The MP and ML bootstrap support values $\geq 50 \%$ and $\mathrm{BYPP} \geq 0.90$ are indicated at the nodes and branches. The tree is rooted with $C$. sansevieriae (MAFF 239721) and C. truncatum (CBS 151.35). 
Table 6 GenBank accession numbers of the species in the boninense complex

\begin{tabular}{|c|c|c|c|c|c|c|c|}
\hline Species name & Type/Reference strain & ITS & gapdh & chs -1 & act & $t u b 2$ & References \\
\hline C. annellatum & CBS 129826 & JQ005222 & JQ005309 & JQ005396 & JQ005570 & JQ005656 & Damm et al. (2012a) \\
\hline C. beeveri & CBS 128527 & JQ005171 & JQ005258 & JQ005345 & JQ005519 & JQ005605 & Damm et al. (2012a) \\
\hline C. boninense & CBS 123755 & JQ005153 & JQ005240 & JQ005327 & JQ005501 & JQ005588 & Damm et al. (2012a) \\
\hline C. brasiliense & CBS 128501 & JQ005235 & JQ005322 & JQ005409 & JQ005583 & JQ005669 & Damm et al. (2012a) \\
\hline C. brassicicola & CBS 101059 & JQ005172 & JQ005259 & JQ005346 & JQ005520 & JQ005606 & Damm et al. (2012a) \\
\hline $\begin{array}{l}\text { C. camelliae- } \\
\text { japonicae }\end{array}$ & CGMCC3.18118 & KX853165 & KX893584 & & KX893576 & KX893580 & Hou et al. (2016) \\
\hline C. catinaense & CBS 142417 & KY856400 & KY856224 & KY856136 & KY855971 & KY856482 & Guarnaccia et al. (2017) \\
\hline C. chiangraiense & MFLUCC 14-0119 & MF448522 & & & MH376383 & MH351275 & Ma et al. (2018) \\
\hline C. citricola & CBS 134228 & KC293576 & KC293736 & KC293792 & KC293616 & KC293656 & Guarnaccia et al. (2017) \\
\hline C. colombiense & CBS 129818 & JQ005174 & JQ005261 & JQ005348 & JQ005522 & JQ005608 & Damm et al. (2012a) \\
\hline C. condaoense & CBS 134299 & MH229914 & MH229920 & MH229926 & & MH229923 & Crous et al. (2018) \\
\hline C. constrictum & CBS 128504 & JQ005238 & JQ005325 & JQ005412 & JQ005586 & JQ005672 & Damm et al. (2012a) \\
\hline C. cymbidiicola & IMI 347923 & JQ005166 & JQ005253 & JQ005340 & JQ005514 & JQ005600 & Damm et al. (2012a) \\
\hline C. dacrycarpi & CBS 130241 & JQ005236 & JQ005323 & JQ005410 & JQ005584 & JQ005670 & Damm et al. (2012a) \\
\hline C. doitungense & MFLUCC 14-0128 & MF448524 & МH049480 & & MH376385 & MH351277 & Ma et al. (2018) \\
\hline C. feijoicola & CBS 144633 & MK876413 & MK876475 & & MK876466 & MK876507 & Crous et al. (2019) \\
\hline C. hippeastri & CBS 125376 & JQ005231 & JQ005318 & JQ005405 & JQ005579 & JQ005665 & Damm et al. (2012a) \\
\hline C. karstii & CORCG6 & HM585409 & HM585391 & HM582023 & HM581995 & HM585428 & Yang et al. (2011) \\
\hline C. limonicola & CBS 142410 & KY856472 & KY856296 & KY856213 & KY856045 & KY856554 & Guarnaccia et al. (2017) \\
\hline C. novae-zelandiae & CBS 128505 & JQ005228 & JQ005315 & JQ005402 & JQ005576 & JQ005662 & Damm et al. (2012a) \\
\hline C. oncidii & CBS 129828 & JQ005169 & JQ005256 & JQ005343 & JQ005517 & JQ005603 & Damm et al. (2012a) \\
\hline C. parsonsiae & CBS 128525 & JQ005233 & JQ005320 & JQ005407 & JQ005581 & JQ005667 & Damm et al. (2012a) \\
\hline C. petchii & CBS 378.94 & JQ005223 & JQ005310 & JQ005397 & JQ005571 & JQ005657 & Damm et al. (2012a) \\
\hline C. phyllanthi & CBS 175.67 & JQ005221 & JQ005308 & JQ005395 & JQ005569 & JQ005655 & Damm et al. (2012a) \\
\hline C. torulosum & CBS 128544 & JQ005164 & JQ005251 & JQ005338 & JQ005512 & JQ005598 & Damm et al. (2012a) \\
\hline C. watphraense & MFLUCC $14-0123$ & MF448523 & MH049479 & & MH376384 & MH351276 & Ma et al. (2018) \\
\hline
\end{tabular}

Notes - This species is sister to a clade that contains C. karstii and C. phyllanthi. Colletotrichum annellatum has longer asci compared with C. karstii, wider ascospores and smaller appressoria (produce rarely) (Damm et al. 2012a). There are no strains recorded after Damm et al. (2012a) of this species.

Colletotrichum beeveri Damm, P.F. Cannon, Crous, P.R. Johnst \& B. Weir, in Damm et al., Stud. Mycol. 73: 9 (2012)

Sexual morph not reported. See Damm et al. (2012a) for illustrations and descriptions of asexual morph.

Type - New Zealand, Great Barrier Island, from brown lesions on a leaf of Brachyglottis repanda, R.E. Beever, 23 Mar. 2006, holotype CBS H20694, culture ex-type CBS 128527. 
Hosts - Brachyglottis repanda (Damm et al. 2012a)

Distribution - New Zealand (Damm et al. 2012a)

Notes - This species is characterised by wide conidia and complex appressoria. It can be distinguished by any of the loci (act, chs-1, tub2, his3 and cal) used in Damm et al. (2012a) except for ITS and gapdh. Even though $C$. beeveri was isolated from leaf lesions, there is no pathogenicity data available to confirm this.

Colletotrichum boninense Moriwaki, Toy. Sato \& Tsuki, Mycoscience 44: 48 (2003)

See Damm et al. (2012a) for illustrations and descriptions of asexual and sexual morphs.

Type - Japan, Bonin Islands, from a diseased leaf of Crinum asiaticum var. sinicum, 1988, T. Sato, culture ex-holotype CBS 123755.

Hosts - Has a wide range of hosts (Amaryllidaceae, Annonaceae, Bignoniaceae, Lauracea, Olivaceae, Orchidaceae, Piperaceae, Podocarpaceae, Protaceae, Rubiaceae, Rutaceae, Solanaceaea and Theaceae) (Damm et al. 2012a, Jayawardena et al. 2016a)

Distribution - Worldwide (Damm et al. 2012a, Jayawardena et al. 2016a, Farr \& Rossman 2021)

Notes - Colletotrichum boninense is an endophyte and a pathogen on a wide range of hosts, worldwide which was introduce by Moriwaki et al. (2003). Damm et al. (2012a) recognized a significant genetic variation in this species.

Colletotrichum brasiliense Damm, P.F. Cannon, Crous \& Massola, in Damm et al., Stud. Mycol. 73: 11 (2012)

Sexual morph not reported. See Damm et al. (2012a) for illustrations and descriptions of asexual morph.

Type - Brazil, Sao Paulo, Bauru City, from fruit anthracnose of Passiflora edulis f. flavicarpa, 1 June 2006, N. Massola and H. J. Tozze Jr., holotype CBS H-20697, culture ex-type CBS 128501.

Hosts - Passiflora edulis (Damm et al. 2012a)

Distribution - Brazil (Damm et al. 2012a)

Notes - Even though this species was isolated as a pathogen from fruit anthracnose, no pathogenicity data is available to confirm this. This species is closely related to C. parsonsiae and C. hippeastri (Damm et al. 2012a). This taxon can be distinguished from the other species with the use of act, gapdh, ITS and tub2 sequence data (Damm et al. 2012a). There are no records of this species after Damm et al. (2012a).

Colletotrichum brassicicola Damm, P.F. Cannon \& Crous, in Damm et al., Stud. Mycol. 73: 14 (2012)

See Damm et al. (2012a) for illustrations and descriptions of asexual and sexual morphs.

Type - New Zealand, Manawatu-Wanganui, Ohakune, from leaf spot of Brassica oleracea var. gemmifera, unknown collection date, holotype CBS H-20698, culture ex-type CBS 101059.

Hosts - Brassica oleracea (Damm et al. 2012a)

Distribution - New Zealand (Damm et al. 2012a)

Notes - The conidia of $C$. brassicicola are very short, while ascospores and asci are longer than those of the other species in the boninense species complex with a known sexual morph (Damm et al. 2012a). It can be distinguished by any of the loci (act, chs-1, tub2, his3 and cal) used in Damm et al. (2012a) except for ITS and gapdh. Even though it was isolated from a leaf spot, no pathogenicity data are available to confirm the pathogenicity of this species. There are no records available for this species other than type strain.

Colletotrichum camellia-japonicae L.W. Hou \& L. Cai, in Hou et al., Mycosphere 7(8): 1117 (2016)

See Hou et al. (2016) for illustrations and descriptions of asexual and sexual morphs. 
Type - Japan, intercepted by Ningbo Entry-Exit Inspection and Quarantine Bureau when exporting to China, on Camellia japonica, Weijun Duan, 25 November 2013, holotype HMAS 247042, culture ex-holotype CGMCC 3.18118.

Hosts - Camellia japonica (Hou et al. 2016)

Distribution - Japan (Hou et al. 2016)

Notes - This taxon is closely related to C. citricola and can be differentiated by its narrow ascospores and shorter conidia (Hou et al. 2016).

Colletotrichum catinaense Guarnaccia \& Crous, in Guarnaccia et al., Persoonia 39: 43 (2017)

Sexual morph not reported. See Guarnaccia et al. (2017) for illustrations and descriptions of asexual morph.

Type - Italy, Mineo, Catania, from leaf lesion of Citrus reticulata, 23 Sept. 2015, V. Guarnaccia, holotype CBS H-23024, culture ex-type CBS 142417.

Hosts - Citrus sp. (Guarnaccia et al. 2017)

Distribution - Italy, Portugal (Guarnaccia et al. 2017)

Notes - Colletotrichum catinaense was isolated from Citrus in Italy and Portugal. This species is phylogenetically close to but clearly differentiated from $C$. novae-zelandiae in cal and tub2 sequence data (Guarnaccia et al. 2017).

Colletotrichum chiangraiense X.Y. Ma, K.D. Hyde \& Jayawardena, in Ma et al., MycoKeys 43: 34 (2018)

See Ma et al. (2018) for illustrations and descriptions of asexual and sexual morphs.

Type - Thailand, Chiang Rai, Wat Phra That Doi Tung, Dendrobium sp. was collected on 19 December 2013, Collector: Sureeporn Nontachaiyapoom, Natdanai Aewsakul, Xiaoya Ma, holotype MFLU 17-1201, culture ex-type MFLUCC 14-0119.

Hosts - Dendrobium sp. (Ma et al. 2018)

Distribution - Thailand (Ma et al. 2018)

Notes - It was isolated as an endophyte and is closely related to C. cymbidiicola. This species may be homothallic (Ma et al. 2018).

Colletotrichum citricola F. Huang, L. Cai, K.D. Hyde \& Hong Y. Li, in Huang et al., Fungal Diversity 61(1): 67 (2013)

See Fu et al. (2019) for illustrations and descriptions of sexual morph. See Huang et al. (2013) for illustrations and descriptions of asexual morph.

Type - China, Shaanxi province, Chenggu, saprobes on leaf of Citrus unshiu, May 2012, F. Huang holotype ZJUC34H, culture ex-type CBS134228.

Hosts - Citrus sp. (Huang et al. 2013, Fu et al. 2019)

Distribution - China (Huang et al. 2013, Fu et al. 2019)

Notes - This species has been reported as a saprobe and a pathogen only from Citrus sp. in China (Huang et al. 2013, Fu et al. 2019). Pathogenicity data are available to for this species. Fu et al. (2019) mentioned that this taxon can be organ-specific (fruit or leaf) on Citrus sp.

Colletotrichum colombiense Damm, P.F. Cannon \& Crous, in Damm et al., Stud. Mycol. 73: 16 (2012)

Sexual morph not reported. See Damm et al. (2012a) for illustrations and descriptions of asexual morph.

Type - Colombia, Cundinamarca, Tibacuy, from a leaf of Passiflora edulis, 22 Jan. 2010, D. Riascos, holotype CBS H-20699, culture ex-type CBS 129818.

Hosts - Passiflora edulis (Damm et al. 2012a)

Distribution - Colombia (Damm et al. 2012a)

Notes - Colletotrichum colombiense forms a sister group to $C$. beeveri and $C$. brassicicola. It differs from $C$. beeveri in morphology by setae that are verrucose and rounded, and shorter 
conidiogenous cells (Damm et al. 2012a). This taxon can be identified by all loci (act, tub2, chs-1, gapdh, his3, cal) used in Damm et al. (2012a) except for ITS.

Colletotrichum conodaoense J.A. Crouch, IMA Fungus 5(1):27 (2014)

See Crous et al. (2018b) for illustrations and descriptions of asexual and sexual morphs.

Type - Vietnam, Côn Đảo Islands, Côn Sơn, sea shore, from leaf spots on Ipomoea pescaprae, 12 Dec. 2012, U. Damm, holotype CBSH-21508, culture ex-holotype CBS 134299.

Hosts - Ipomoea pes-caprae (Crous et al. 2018b)

Distribution - Vietnam (Crous et al. 2018b)

Notes - This taxon is closely related to $C$. parsoniae and can be differentiated by its appresorial and ascospore shapes (Damm et al. 2012). Even though this species was isolated from a leaf spot, no data are available to confirm its pathogenicity.

Colletotrichum constrictum Damm, P.F. Cannon, Crous, P.R. Johnst \& B. Weir, in Damm et al., Stud. Mycol. 73: 17 (2012)

See Damm et al. (2012) for illustrations and descriptions of asexual and sexual morphs.

Type - New Zealand, AK, Auckland, from fruit of Citrus limon, 1 Dec. 1988, P.R. Johnston, holotype CBS H-20701, culture ex-type CBS 128504.

Hosts - Citrus limon and Solanum betacum (Damm et al. 2012a)

Distribution - New Zealand (Damm et al. 2012a)

Notes - This taxon differs by the shape and size of the ascospores, which are broader than those of the other species (Damm et al. 2012a). Even though this species is isolated from fruit rot, no pathogenicity data are available to confirm its pathogenicity. This taxon seems to be endemic to New Zealand.

Colletotrichum cymbidiicola Damm, P.F. Cannon, Crous, P.R. Johnst. \& B. Weir, in Damm et al., Stud. Mycol. 73: 19 (2012)

See Damm et al. (2012a) for illustrations and descriptions of asexual and sexual morphs.

Type - Australia, Western Australia, Perth, Fremantle, from leaf lesion of Cymbidium sp., 27 Mar. 1991, P.M. Wood, holotype CBS H-20703, culture extype IMI 347923.

Hosts - Cymbidium sp. (Damm et al. 2012a), Bulbophyllum hirtum, Coelogyne elata, Dendrobium fimbriatum, Eria sp., Liparis longipes, Oncidium sphacelatum (Chowdappa et al. 2014)

Distribution - Australia, Japan (Damm et al. 2012a), India (Chowdappa et al. 2014), China (Liu et al. 2018)

Notes - Colletotrichum cymbidiicola differs from $C$. boninense in the shape of the appressoria that are usually lobate with irregular shapes in $C$. cymbidiicola, while those of $C$. boninense are typically bullet-shaped to navicular with entire edge or crenate (Damm et al. 2012a). In her study Damm et al. (2012a) mentioned that this taxon seems to be host-specific at the plant genus level.

Colletotrichum dacrycarpi Damm, P.F. Cannon, Crous, P.R. Johnst. \& B. Weir, in Damm et al., Stud. Mycol. 73: 19 (2012)

Sexual morph not reported. See Damm et al. (2012a) for illustrations and descriptions of asexual morph.

Type - New Zealand, Auckland, Wenderholm Regional Park, leaf endophyte from Dacrycarpus dacrydioides, 16 Oct. 2009, G. Carroll, holotype CBS H-20705, culture ex-type CBS 130241.

Hosts - Dacrycarpus sp. (Damm et al. 2012a)

Distribution - New Zealand (Damm et al. 2012a)

Notes - Colletotrichum dacrycarpi does not look like a typical member of Colletotrichum, with its slow growth and the production of conidia within closed fruit-bodies with walls that 
rupture (Damm et al. 2012a). It is one of the most basal members of the boninense complex. There are no records of this species other than the ex-type strain.

Colletotrichum doitungense X.Y. Ma, K.D. Hyde \& Jayawardena, in Ma et al., MycoKeys 43: 37 (2018)

See Ma et al. (2018) for illustrations and descriptions of asexual and sexual morphs.

Type - Thailand, Chiang Rai, Wat Phra That Doi Tung, Dendrobium sp., 19 December 2013, Collector: Sureeporn Nontachaiyapoom, Natdanai Aewsakul, Xiaoya Ma, holotype MFLU 171200, culture ex-type MFLUCC 14-0128.

Hosts - Dendrobium sp. (Ma et al. 2018)

Distribution - Thailand (Ma et al. 2018)

Notes - This taxon was isolated as an endophyte from the root of a Dendrobium sp. It can be distinguished by its globose to sub-globose conidiogenous cell (Ma et al. 2018).

Colletotrichum feijoicola Guarnaccia \& Damm, in Crous et al., Persoonia 42: 401 (2019)

Sexual morph not reported. See Crous et al. (2019) for illustrations and descriptions of asexual morph.

Type - Portugal, Azores Islands, Sao Miguel, from a leaf spot of Acca sellowiana, 17 July 2017, V. Guarnaccia, holotype GML-F116096, culture ex-type CBS 144633.

Hosts - Acca sellowiana (Crous et al. 2019)

Distribution - Portugal (Crous et al. 2019)

Notes - Colletotrichum feijoicola was found associated with reddish leaf spots, however no pathogenicity data are available to confirm its pathogenicity (Crous et al. 2019). This taxon can be separated from act, gapdh and tub2 sequence data.

Colletotrichum hippeastri Yan L. Yang, Zuo Y. Liu, K.D. Hyde \& L. Cai, in Yang et al., Fungal Diversity 39: 133. 2009

Sexual morph not reported. See Damm et al. (2012a) for illustrations and descriptions of asexual morph.

Type - China, Guizhou Province, Guiyang, isolated from leaf of Hippeastrum vittatum, 23 May 2009, Y.L. Yang, culture ex-holotype CBS 125376.

Hosts - Hippeastrum sp. (Damm et al. 2012a)

Distribution - China, Netherland (Damm et al. 2012a)

Notes - Colletotrichum hippeastri is an outlying species in the boninense clade and is distinguished from related species by its large conidia as well as elongate and complex appressoria (Damm et al. 2012a). This endophytic species can be distinguished by any of the loci (ITS, act, tub2, chs-1, gapdh, his3 and cal) used in Damm et al. (2012a).

Colletotrichum karstii Y.L. Yang, Zuo Y. Liu, K.D. Hyde \& L. Cai, in Yang et al., Cryptogamie Mycologie 32: 241. 2011.

See Damm et al. (2012a) for illustrations and descriptions of asexual and sexual morphs.

Type - China, Guizhou Province, Luodian, on leaf of Vanda sp., 15 August 2009, Y.L. Yang, holotype GZAAS 090006, culture ex-holotype CGMCC3.14194.

Hosts - wide range of hosts (including Annona cherimola, Anthurium sp., Bletilla ochracea, Bombax aquaticum, Camellia sp., Capsicum annunum, Carica papaya, Citrus sp., Clivia miniata, Coffea sp., Cucumis melo, Diospyros australis, Eucalyptus grandis, Malus domestica, Mangifera indica, Olea europaea, Pistacia vera, Quercus salicifolia, Rubus glaucus, Theobroma cacao, Zamia oblique) (Damm et al. 2012a, Jayawardena et al. 2016a, Farr \& Rossman 2021)

Distribution - Australia, Brazil, China, Colombia, Germany, India, Italy, Japan, Mexico, New Zealand, Panama, Portugal, South Africa, Spain, Thailand, USA, Zimbabwe (Damm et al. 2012a, Jayawardena et al. 2016a, Farr \& Rossman 2021)

Notes - This is the most common and geographically diverse species in the boninense 
complex (Damm et al. 2012a, Jayawardena et al. 2016a). Morphological and genetic variation of this species is very high, which makes identification difficult. Pathogenicity studies for this species are available.

Colletotrichum limonicola Guarnaccia \& Crous, in Guarnaccia et al., Persoonia 39: 45 (2017)

Sexual morph not reported. See Guarnaccia et al. (2017) for illustrations and descriptions of asexual morph.

Type - Malta, Gozo, from wither-tip twigs of Citrus limon, 11 July 2016, V. Guarnaccia, holotype CBS H-23027, culture ex-type CBS 142410.

Hosts - Citrus limon (Guarnaccia et al. 2017)

Distribution - Malta (Guarnaccia et al. 2017)

Notes - Colletotrichum limonicola was isolated from leaf lesions and twigs with wither-tip symptoms. This species is phylogenetically closer to but clearly differentiated from $C$. novaezelandiae based on gapdh and tub2 sequence data.

Colletotrichum novae-zelandiae Damm, P.F. Cannon, Crous, P.R. Johnst. \& B. Weir, in Damm et al., Stud. Mycol. 73: 25 (2012)

Sexual morph not reported. See Damm et al. (2012a) for illustrations and descriptions of asexual morph.

Type - New Zealand, GB, Gisborne, from ripe fruit rot of Capsicum annuum, 1 Mar. 1990, P.R. Johnston, holotype CBS H-20706, culture ex-type CBS 128505.

Hosts - Capsicum sp., Citrus sp. (Damm et al. 2012a, Guarnaccia et al. 2017)

Distribution - New Zealand (Damm et al. 2012a), Greece, Iran, Malta (Guarnaccia et al. 2017)

Notes - This species was thought to be endemic to New Zealand (Jayawardena et al. 2016a) until more strains from European countries were discovered. Colletotrichum novae-zelandiae can be distinguished by any of the loci (ITS, act, tub2, chs-1, gapdh, his3 and cal) used in Damm et al. (2012a).

Colletotrichum oncidii Damm, P.F. Cannon \& Crous, in Damm et al., Stud. Mycol. 73: 26 (2012)

Sexual morph not reported. See Damm et al. (2012a) for illustrations and descriptions of asexual morph.

Type - Germany, Munich, greenhouse, from leaf of Oncidium sp., 20 Nov. 2010, U. Damm, holotype, CBS H-20709, culture ex-type CBS 129828.

Hosts - Oncidium sp (Damm et al. 2012a)

Distribution - Germany (Damm et al. 2012a)

Notes - Colletotrichum oncidii differs from the closely related $C$. boninense in forming appressoria that are larger and lobate (Damm et al. 2012a). This species is only recorded from Germany. As it was isolated from a green house, the origin is unknown.

Colletotrichum parsonsiae Damm, P.F. Cannon, Crous, P.R. Johnst. \& B. Weir, in Damm et al., Stud. Mycol. 73: 27 (2012)

See Damm et al. (2012a) for illustrations and descriptions of asexual and sexual morphs.

Type - New Zealand, Auckland, leaf endophyte from Parsonsia capsularis, 1 Dec. 2009, G. Carroll, (CBS H-20710 holotype, culture ex-type CBS 128525.

Hosts - Parsonsia capsularis (Damm et al. 2012a), Bletilla ochracea (Tao et al. 2013)

Distribution - New Zealand (Damm et al. 2012a), China (Tao et al. 2013)

Notes - This species was introduced based on a single strain (Damm et al. 2012a). Tao et al. (2013) provided another host for this taxon from China. The shape and size of conidia differ from other species in the boninense complex (Damm et al. 2012a).

Colletotrichum petchii Damm, P.F. Cannon \& Crous, in Damm et al., Stud. Mycol. 73: 29 (2012) 
See Damm et al. (2012a) for illustrations and descriptions of asexual and sexual morphs.

Type - Italy, from spotted leaves of Dracaena fragrans, P. Di Lenna (from Università degli 378.94

Studi, Padova), deposited in June 1994, epitype CBS-H 20711, culture ex-epitype CBS

Hosts - Dracaena sp. (Damm et al. 2012a, Shivas et al. 2016)

Distribution - China, Germany, Italy, Netherlands, Sri Lanka (Damm et al. 2012a), Australia (Shivas et al. 2016)

Notes - This species is recorded as a pathogen. However, no pathogenicity data are available to confirm its pathogenicity. Within the species there is only low sequence variability and can be identified by any of the loci (ITS, act, tub2, chs-1, gapdh, his3 and cal) used in Damm et al. (2012a).

Colletotrichum phyllanthi (H.S. Pai) Damm, P.F. Cannon \& Crous, in Damm et al., Stud. Mycol. 73: 31 (2012)

See Damm et al. (2012a) for illustrations and descriptions of asexual and sexual morphs.

Type - India, Maharashtra, Poona, isolated from anthracnose symptoms on leaves of Phyllanthus acidus, 10 Feb. 1966, H. Surendranath Pai, isotype CBS H-7188 (dried culture (PDA) of ascigerous stage), culture ex-isotype CBS 175.67.

Hosts - Phyllanthus acidus (Damm et al. 2012a), Bauhinia variegate, Bougainvillea glabra (Sharma \& Shenoy 2013)

Distribution - India (Damm et al. 2012a, Sharma \& Shenoy 2013)

Notes - This species is recorded as an endophyte and a pathogen from India. Colletotrichum phyllanthi can be distinguished by any of the loci (ITS, act, tub2, chs-1, gapdh, his3 and cal) used in Damm et al. (2012a).

Colletotrichum torulosum Damm, P.F. Cannon, Crous, P.R. Johnst. \& B. Weir, in Damm et al., Stud. Mycol. 73: 32 (2012)

Sexual morph not reported. See Damm et al. (2012a) for illustrations and descriptions of asexual morph.

Type - New Zealand, GB, Gisborne, Allen Park Gardens, from Solanum melongena, 6 Mar. 1990, P.R. Johnston, holotype CBS H-20715, culture ex-type CBS 128544.

Hosts - Passiflora edulis, Solanum melongena (Damm et al. 2012a)

Distribution - New Zealand (Damm et al. 2012a)

Notes - It has significantly longer conidia than $C$. boninense with a larger L/W ratio (Damm et al. 2012a). This species is recorded only from New Zealand. Damm et al. (2012a) mentioned that it is not clear whether this species is native to New Zealand, and has jumped onto cultivated exotic plants or has been imported on diseased plant materials.

Colletotrichum watphraense X.Y. Ma, K.D. Hyde \& Jayawardena, in Ma et al., MycoKeys 43: 35 (2018)

Sexual morph not reported. See Ma et al. (2018) for illustrations and descriptions of asexual morph.

Type - Thailand, Chiang Rai, Wat Phra That Doi Tung, Dendrobium sp., 19 December 2013, Collector: Sureeporn Nontachaiyapoom, Natdanai Aewsakul, Xiaoya Ma, holotype MFLU 171202, culture ex-type 14-0123.

Hosts - Dendrobium sp. (Ma et al. 2018)

Distribution - Thailand (Ma et al. 2018)

Notes - It was isolated from the stem of Dendrobium sp. as an endophyte and can be distinguished by its closely related taxa with any of the loci (ITS, act, tub2, chs-1, and gapdh) used in Ma et al. (2018).

\section{Graminicola-caudatum Species Complex}


This species complex is defined as a collective of $C$. caudatum and $C$. graminicola species and 23 closely related species. Crouch (2014) introduced the caudatum complex based on the presence of a filiform appendage at the apex of the conidium and phylogenetic analyses (apn2, ITS, Sod2, Mat/apn2). A phylogenetic tree using ITS for the species of caudatum and graminicola species complexes was generated in Jayawardena et al. (2016a). According to this phylogenetic tree, caudatum complex appears to be a specific branch within the graminicola complex. Three species viz. C. caudasporum, $C$. duyuensis and $C$. ochracea which were previously identified to be in the graminicola species complex, claded with the caudatum species complex. Similar phylogenetic placement was observed in the later studies (Marin-Felix et al. 2017, Jayawardena et al. 2020). Both species complexes are from monocotyledons, mostly being from Poaceae. Bhunjun et al. (2021) based on phylogenetic and evolutionary analyses recommended to treat these two complexes as one and we follow this herein. Almost all of the species are either host-specific (Poaceae) or geographically restricted. However, for some of these species only a single strain is available. Therefore, new collections are recommended to establish the host distribution of these species. A combined gene analysis for this complex using ITS, gapdh, chs-1, act and tub2 sequence data is given in Fig. 8, Table 7.

Colletotrichum alcornii J.A. Crouch, IMA Fungus 5(1):27 (2014)

Sexual morph not reported. See Crouch (2014) for illustrations and descriptions of asexual morph.

Type - Australia: Queensland: Caboolture, on leaves of Imperata cylindrica var. major, 26 March 1973, J. L. Alcorn holotype IMI 176619

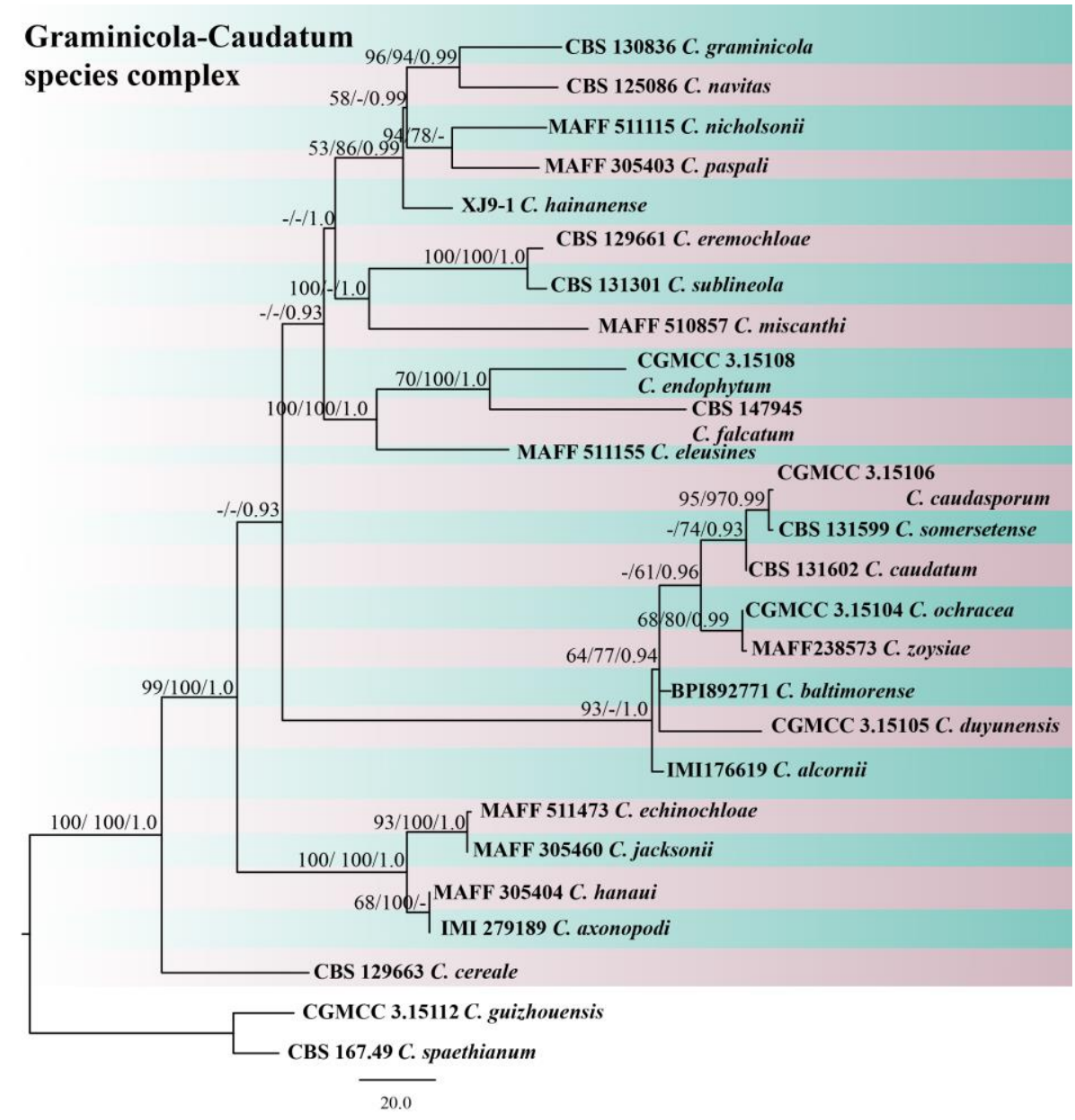

Figure 8 The graminicola-caudatum complex. One of the 10 most parsimonious trees obtained from a heuristic search of combined ITS, gapdh, chs-1, act and tub2 sequence data. The MP and 
ML bootstrap support values $\geq 50 \%$ and $\mathrm{BYPP} \geq 0.90$ are indicated at the nodes and branches. The tree is rooted with $C$. guizhouensis (CGMCC 3.15112) and C. spaethianum (CBS 167.49).

Table 7 GenBank accession numbers of species in the graminicola/caudatum complex

\begin{tabular}{|c|c|c|c|c|c|c|c|}
\hline Species name & Type/Reference strain & ITS & gapdh & chs-1 & act & tub2 & References \\
\hline C. alcornii & IMI176619 & JX076858 & & & & & Crouch (2014) \\
\hline C. axonopodi & IMI 279189 & MN521699 & & & & & Zhang et al. (2020) \\
\hline C. baltimorense & BPI892771 & JX076866 & & & & & Crouch (2014) \\
\hline C. caudatum & CBS 131602 & JX076860 & & & & & Crouch (2014) \\
\hline C. caudasporum & CGMCC 3.15106 & JX625162 & KC843512 & & KC843526 & JX625190 & Tao et al. (2013) \\
\hline C. cereale & CBS 129663 & & & JQ005795 & JQ005837 & JQ005858 & Crouch et al. (2006) \\
\hline C. duyunensis & CGMCC 3.15105 & JX625160 & KC843515 & & KC 843530 & JX625187 & Tao et al. (2013) \\
\hline C. echinochloae & MAFF 511473 & AB439811 & & & & & Moriwaki \& Tsukiboshi (2009) \\
\hline C. eleusines & MAFF 511155 & JX519218 & & JX519226 & JX519234 & JX519243 & Crouch et al. (2009a) \\
\hline C.endophytum & CGMCC 3.15108 & JX625177 & KC843521 & & KC843533 & JX625206 & Tao et al. (2013) \\
\hline C. eremochloae & CBS 129661 & JX519220 & & JX519228 & JX519236 & JX519245 & Crouch \& Tomaso-Peterson (2012) \\
\hline C. falcatum & CBS 147945 & JQ005772 & & JQ005793 & JQ005835 & JQ005856 & Prihastuti et al. (2010) \\
\hline C. graminicola & M 1.001/CBS 130836 & JQ005767 & & JQ005788 & JQ005830 & JQ005851 & Du et al. (2005) \\
\hline C. hanaui & MAFF 305404 & JX519217 & & JX519225 & & JX519242 & Crouch et al. (2009a) \\
\hline C. jacksonii & MAFF 305460 & JX519216 & & JX519224 & JX519233 & JX519241 & Crouch et al. (2009a) \\
\hline C. miscanthi & MAFF 510857 & JX519221 & & JX519229 & JX519237 & JX519246 & Crouch et al. (2009c) \\
\hline C. navitas & CBS 125086 & JQ005769 & & JQ005790 & JQ005832 & JQ005853 & Crouch et al. (2009a) \\
\hline C. nicholsonii & MAFF 511115 & JQ005770 & & JQ005791 & JQ005833 & JQ005854 & Crouch et al. (2009a) \\
\hline C. ochracea & CGMCC 3.15104 & JX625168 & KC843513 & & KC 843527 & JX625183 & Tao et al. (2013) \\
\hline C. paspali & MAFF 305403 & JX519219 & & JX519227 & JX519235 & JX519244 & Crouch et al. (2009a) \\
\hline C. somersetense & CBS 131599 & JX076862 & & & & & Crouch (2014) \\
\hline C. sublineola & CBS 131301, S3.001 & JQ005771 & & JQ005792 & JQ005834 & JQ005855 & Crouch et al. (2006) \\
\hline C. zoysiae & MAFF238573 & JX076871 & & & & & Crouch (2014) \\
\hline
\end{tabular}

Hosts - Bothriochloa bladhii and Imperata cylindrica (Crouch 2014)

Distribution - Australia (Crouch 2014)

Notes - Colletotrichum alcornii was introduced in the caudatum species complex and can be distinguished by any of the loci (apn2, ITS, mat and sod2) used in Crouch (2014). This taxon appears to be endemic to Australia.

Colletotrichum axonopodi J.A. Crouch, B.B. Clarke, J.F. White \& B.I. Hillman [as ‘axonopodi’], Mycologia 101(5): 727 (2009)

Sexual morph not reported. See Crouch et al. (2009a) for illustrations and descriptions of asexual morph. 
Type - Italy, Pavia, Ticinum, in botanical garden, on decaying leaves of cultivated Agave americana, 1904, Hedgcock G. holotype BPI 397108, culture ex-type CBS 118190.

Hosts/substrates - Axonopus spp. (Crouch et al. 2009a)

Distribution - Australia, USA (Crouch et al. 2009a)

Notes - Colletotrichum axonopodi cannot be distinguished from the majority of falcatespored species described from graminicolous hosts (Crouch et al. 2009a). This species was listed as a singleton species in Jayawardena et al. (2016a, 2020) and Marlin-Felix et al. (2017). Bhunjun et al. (2021) based on a polyphasic approach identified that this species claded within the graminicolacaudatum complex. Therefore, we follow this herein.

Colletotrichum baltimorense J.A. Crouch, IMA Fungus 5(1): 27 (2014) morph.

Sexual morph not reported. See Crouch (2014) for illustrations and descriptions of asexual

Type - USA: Maryland: Baltimore County, Owings Mills, Natural Environment Area, on leaves of Sorghastrum nutans, 12 September 2011, J. A. Crouch, holotype BPI I1892771 holotype, culture ex-holotype SD-11.

Hosts - Sorghastrum nutans (Crouch 2014)

Distribution - USA (Crouch 2014)

Notes - Colletotrichum baltimorense was introduced in the caudatum species complex and can distinguish by any of the loci (apn2, ITS, mat and sod2) used in Crouch (2014). This taxon is only recorded from Sorghastrum nutans in USA.

Colletotrichum caudatum (Peck ex Sacc.) Peck, Bull. N.Y. State Mus. 131: 81 (1909). morph.

Sexual morph not reported. See Crouch (2014) for illustrations and descriptions of asexual

Type - USA, New York: Big Flats, Chemung Co., on the leaves of Sorghastrum nutans 'Rumsey', 2007, G. C. Bergstom, epitype BPI 892767, culture ex-epitype CBS 131602.

Hosts - Sorghastrum nutans (Crouch 2014)

Distribution - USA (Crouch 2014)

Notes - This taxon was introduced in the caudatum species complex and can be distinguished by any of the loci (apn2, ITS, mat and sod2) used in Crouch (2014). Colletotrichum caudatum appears to be host-specific and geographically restricted to USA. Whole-genome data are available for this species (https://mycocosm.jgi.doe.gov/Colletotrichum/Colletotrichum.info.html).

Colletotrichum caudisporum G. Tao, Zuo Y. Liu \& L. Cai [as 'caudasporum'], in Tao et al., Fungal Diversity 61: 149 (2013)

Sexual morph not reported. See Tao et al. (2013) for illustrations and descriptions of asexual morph.

Type - China, Guizhou Province, Duyun, Xiaba Mountain, isolated from healthy leaves of Bletilla ochracea, 13 July 2006, Gang Tao, holotype HMAS 244282 (dried culture), culture exholotype CGMCC 3.15106.

Hosts - Bletilla ochracea (Tao et al. 2013)

Distribution - China (Tao et al. 2013)

Notes - Colletotrichum caudisporum is introduced as an endophyte from Bletilla ochracea (Tao et al. 2013). This species clustered within the caudatum complex (Jayawardena et al. 2016a).

Colletotrichum cereale Manns, in Selby \& Manns, Proc. Indiana Acad. Sci.: 111 (1908)

Sexual morph not reported. See Crouch et al. (2006) for illustrations and descriptions of asexual morph.

Type - USA, Kansas, on Bromus inermis, reference strain (KS-20BIG) CBS 12966.

Hosts - Pooideae (Crouch et al. 2006, Jayawardena et al. 2016a), Bletilla ochracea (Tao et al. 2013) 
Distribution - Germany, Japan, New Zealand, Netherlands, USA (Crouch et al. 2006, Jayawardena et al. 2016a), China (Tao et al. 2013)

Notes - Colletotrichum cereale is the pathogen of grass of the subfamily Pooideae. This taxon has been identified as an endophyte from Bletilla ochracea, which extends its host range. Pathogenicity, evolutionary, whole-genome and infection data are available for this species.

Colletotrichum duyunensis G. Tao, Zuo Y. Liu \& L. Cai, in Tao et al., Fungal Diversity 61: 149 (2013)

Sexual morph not reported. See Tao et al. (2013) for illustrations and descriptions of asexual morph.

Type - China, Guizhou Province, Duyun, Xiaba Mountain, isolated from healthy leaves of Bletilla ochracea, 13 July 2006, Gang Tao, holotype HMAS 244832(dried culture), culture exholotype CGMCC 3.15105.

Hosts - Bletilla ochracea (Tao et al. 2013)

Distribution - China (Tao et al. 2013)

Notes - This taxon was isolated as an endophyte from Bletilla ochracea and is the only existing record to date (Tao et al. 2013). Jayawardena et al. (2016a) treated this taxon in the caudatum species complex.

Colletotrichum echinochloae Moriwaki \& Tsukib., Mycoscience 50(4): 275 (2009)

Sexual morph not reported. See Moriwaki \& Tsukiboshi (2009) for illustrations and descriptions of asexual morph.

Type - Japan, Tsukuba, in Herbario Instituti Nationalis Agro-Environmentalis Scientiae, from leaves of Echinochloae utilis, Senbonmatsu, Nasushiobara-shi, Tochigi Pref., Japonia, 17 Sep. 2007, T. Tsukiboshi, holotype NIAES 20584 (dried culture), culture ex-type MAFF 511473.

Hosts - Echinochloa sp. (Moriwaki \& Tsukiboshi 2009)

Distribution - Japan (Moriwaki \& Tsukiboshi 2009)

Notes - Colletotrichum echinocholae is a pathogen on Echinichloa sp. causing leaf spots (Moriwaki \& Tsukiboshi 2009). No pathogenicity data are available for this taxon. Colletotrichum echinochloae is characterized by its falcate and rather short conidia (Moriwaki \& Tsukiboshi 2009).

Colletotrichum eleusines Pavgi \& U.P. Singh [as 'eleusinis'], Mycopath. Mycol. appl. 27: 85 (1965)

Sexual morph not reported. See Crouch et al. (2009a) for illustrations and descriptions of asexual morph.

Type - Japan, from leaves of Elusines sp., unknown collection date and collector, culture exepitype MAFF 511155.

Hosts - Elusines sp. (Crouch et al. 2009a)

Distribution - Japan, USA (Crouch et al. 2009a)

Notes - It is indistinguishable from the other closely related falcate-spored, grass inhabiting Colletotrichum species. However, any loci (apn2, ITS, mat and sod2) used in Crouch et al. (2009a) can be used to distinguish this taxon. Colletotrichum eleusines appears to be host-specific.

Colletotrichum endophytum G. Tao, Zuo Y. Liu \& L. Cai, in Tao et al., Fungal Diversity 61(1): 152 (2013)

Sexual morph not reported. See Tao et al. (2013) for illustrations and descriptions of asexual morph.

Type - China, Guizhou Province, Shuicheng, Baijipo Mountain, isolated from healthy leaves of Bletilla ochracea, 28 June 2006, Gang Tao, holotype HMAS 244280 (dried culture), culture exholotype CGMCC 3.15108 .

Hosts - Bletilla ochracea (Tao et al. 2013)

Distribution - China (Tao et al. 2013) 
Notes - Colletotrichum endophytum is an endophyte, closely related to C. falcatum (Tao et al. 2013). This taxon can be separated by producing strong falcate, shorter conidia.

Colletotrichum eremochloae J.A. Crouch \& Tomaso-Pet., Mycologia 104(5): 1092 (2012)

Sexual morph not reported. See Crouch \& Tomaso-Peterson (2012) for illustrations and descriptions of asexual morph.

Type - USA, from diseased leaf tissue of Eremochloa ophiuroides in Hattiesburg, Mississippi, 29 Mar 2007, C. Hagstrom, holotype BPI882561, culture ex-type CBS 129661.

Hosts - Eremochloae ophiuroides (Crouch \& Tomaso-Peterson 2012)

Distribution - China, USA (Crouch \& Tomaso-Peterson 2012)

Notes - Colletotrichum eremecholae is a pathogen isolated from Eremochloa ophiuroides. Crouch \& Tomaso-Peterson (2012) provided pathogenicity data to confirm its pathogenicity. This taxon appears to be host-specific. Whole genomic data are available for this species (https://mycocosm.jgi.doe.gov/Colletotrichum/Colletotrichum.info.html).

Colletotrichum falcatum Went, Archiv, voor de Java Suekerrind. 1: 265 (1893)

Sexual morph not reported. See Prihastuti et al. (2010) for illustrations and descriptions of asexual morph.

Type - Indonesia, Central Java, Comal, Gedheg Village on leaves of Saccharum officinarum, 23 March 2008, N.F. Wulandari, neo-type HMAS 240681, culture ex-neotype CGMGC 3.14187.

Hosts - Saccharum officinarum (Prihastuti et al. 2010)

Distribution - Australia, Indonesia, Japan, Netherlands, Thailand (Prihastuti et al. 2010)

Notes - It is a well-known pathogen on Saccharum officinarum. Pathogenicity studies for this species are available. This taxon is in the graminicola clade and can be identified by any of the loci (ITS, act, chs-1 and tub2) used in Prihastuti et al. (2010). Whole-genome data for this species are available (https://mycocosm.jgi.doe.gov/Colletotrichum/Colletotrichum.info.html).

Colletotrichum graminicola (Ces.) G.W. Wilson, Phytopathology 4: 110 (1914)

Sexual morph not reported. See Damm et al. (2009) for illustrations and descriptions of asexual morph.

Type - USA, from Zea mays, unknown collector, 1978, culture ex-epitye CBS 130836.

Hosts - Zea mays (Crouch et al. 2009a)

Distribution - worldwide (Farr \& Rossman 2021)

Notes - Colletotrichum graminicola is a well-known pathogen of Zea mays (Crouch et al. 2009a), belonging to the graminicola clade. Whole-genome sequencing of this species has been completed (O'Connell et al. 2012). Pathogenicity and infection studies are available for this taxon.

Colletotrichum hanaui J.A. Crouch, B.B. Clarke, J.F. White \& B.I. Hillman, Mycologia 101(5): 728 (2009)

Sexual morph not reported. See Crouch et al. (2009a) for illustrations and descriptions of asexual morph.

Type - Japan, Ibaraki 305-8602, Tsukuba, Kannondai, from Digitaria ciliaris, 1975, holotype MAFF 305404 (dried culture).

Hosts - Digitaria ciliaris (Crouch et al. 2009a)

Distribution - China, Japan, USA (Crouch et al. 2009a)

Notes - Colletotrichum hanaui is in the graminicola clade and seems to only occur on Digitaria sp. This species can be distinguished by any of the loci (apn2, ITS, mat and sod2) used in Crouch et al. (2009a), Zhao et al. (2012) showed that this species can be used as a bio-control agent on Digitaria sanguinalis.

Colletotrichum hainanense Wu Zhang \& X.L. Niu, in Zhang et al., Pl. Dis. 104(6): 1747 (2020)

Sexual morph not reported. See Zhang et al. (2020) for illustrations and descriptions of 
asexual morph.

Type - China, Hainan Province, Haikou City, Axonopus compressus (leaf anthracnose), $18^{\text {th }}$ December 2015, W. Zhang, holotype BJFC 010 (dried culture), culture extype CBS 145900.

Hosts - Axonopus compressus (Zhang et al. 2020)

Distribution - China (Zhang et al. 2020)

Notes: Colletotrichum hainanense is in the graminicola clade and can be identified by all loci (ITS, sod2, apn2, apn2/Mat) used in Zhang et al. (2020). This species differs from C. axonopodi by having white to pale gray in center and mouse gray towards the margin on PDA.

Colletotrichum jacksonii J.A. Crouch, B.B. Clarke, J.F. White \& B.I. Hillman, Mycologia 101(5): 729 (2009)

Sexual morph not reported. See Crouch et al. (2009a) for illustrations and descriptions of asexual morph.

Type - Japan, a strain originally isolated from Echinochloa esculenta in the Tochigi Prefecture in 1980, National Institute of Agrobiological Sciences, 2-1-2 Kannondai, Tsukuba, Ibaraki 305-8602, MAFF305460.

Hosts - Echinochloa esculenta (Crouch et al. 2009a)

Distribution - Japan (Crouch et al. 2009a)

Notes - This species was isolated from diseased Echinochloa esculenta, however, no pathogenicity data are available to confirm this. This species clade in the graminicola clade and can be identified by any of the loci (apn2, ITS, mat and sod2) used in Crouch et al. (2009).

Colletotrichum miscanthi J.A. Crouch, B.B. Clarke, J.F. White \& B.I. Hillman, Mycologia 101(5): 729 (2009)

Sexual morph not reported. See Crouch et al. (2009a) for illustrations and descriptions of asexual morph.

Type - Japan, a strain originally isolated from the Tochigi Prefecture in 1972, National Institute of Agrobiological Sciences, 2-1-2 Kannondai, Tsukuba, Ibaraki 305-8602, MAFF510857.

Hosts - Miscanthus sinensis (Crouch et al. 2009a), Bletilla ochracea (Tao et al. 2013)

Distribution - Japan (Crouch et al. 2009a), China (Tao et al. 2013)

Notes - Colletotrichum miscanthi is in the graminicola clade. It was initially thought to be host-specific. Tao et al. (2013) recorded this taxon as an endophyte from Bletilla ochracea.

Colletotrichum navitas J.A. Crouch, Mycol. Res. 113(12): 1417 (2009)

Sexual morph not reported. See Crouch et al. (2009c) for illustrations and descriptions of asexual morph.

Type - United States, from the stem of Panicum virgatum selection 'Brooklyn'in Freehold, NJ, 26 Sept. 2008, holotype 9038-158a culture ex-type CBS 125086.

Hosts - Panicum sp. (Crouch et al. 2009c)

Distribution - USA (Crouch et al. 2009c)

Notes - Colletotrichum navitas is recorded as a pathogen on Panicum sp., and its pathogenicity has been confirmed by Kochs' postulates (Crouch et al. 2009c). It is in the graminicola clade and can be identified using any of the loci (apn2, ITS, mat and sod2) in Crouch et al. (2009c). Whole genomic data for this species are available

(https://mycocosm.jgi.doe.gov/Colletotrichum/Colletotrichum.info.html).

Colletotrichum nicholsonii J.A. Crouch, B.B. Clarke, J.F. White \& B.I. Hillman, Mycologia 101(5): 730 (2009)

Sexual morph not reported. See Crouch et al. (2009a) for illustrations and descriptions of asexual morph.

Type - Japan, a strain originally isolated from Paspalum dilatatum from an unknown location, National Institute of Agrobiological Sciences, 2-1-2 Kannondai, Tsukuba, Ibaraki 305- 


\section{2, MAFF511115.}

Hosts - Paspalum dilatatum (Crouch et al. 2009a)

Distribution - Japan, New Zealand (Crouch et al. 2009a)

Notes - Colletotrichum nicholsonii causes anthracnose on Paspalum sp. in Japan and New Zealand (Crouch et al. 2009a). This species belongs to the graminicola clade and seems to be hostspecific.

Colletotrichum ochraceae G. Tao, Zuo Y. Liu \& L. Cai [as 'ochracea'], in Tao et al., Fungal Diversity 61: 156 (2013)

Sexual morph not reported. See Tao et al. (2013) for illustrations and descriptions of asexual morph.

Type - China, Guizhou Province, Duyun, Xiaba Mountain, isolated from healthy leaves of Bletilla ochracea, 13 July 2006, Gang Tao, holotype HMAS244831 (dried culture), culture exholotype CGMCC. 3.15104.

Hosts - Bletilla ochracea (Tao et al. 2013)

Distribution - China (Tao et al. 2013)

Notes - Being in the caudatum complex, this species is closely related to C. duyunensis, which was also isolated from the same host (Tao et al. 2013).

Colletotrichum paspali J.A. Crouch, B.B. Clarke, J.F. White \& B.I. Hillman, Mycologia 101(5): 730 (2009)

Sexual morph not reported. See Crouch et al. (2009a) for illustrations and descriptions of asexual morph.

Type - Japan, a strain originally isolated from Paspalum notatum from the Yamaguchi Prefecture in 1977, National Institute of Agrobiological Sciences, 2-1-2 Kannondai, Tsukuba, Ibaraki 305-8602, MAFF305403.

Hosts - Paspalum notatum (Crouch et al. 2009a)

Distribution - Japan (Crouch et al. 2009a)

Notes - This species was isolated as a pathogen from Paspalum notatum, however no pathogenicity data are available to confirm this. Colletotrichum paspali is in the graminicola clade.

Colletotrichum somersetense J.A. Crouch, IMA Fungus 5(1):27 (2014) morph.

Sexual morph not reported. See Crouch (2014) for illustrations and descriptions of asexual

Type - USA: New Jersey: Somerset County, Somerset, on the leaves of Sorghastrum nutans, 23 Sept. 2011, J. A. Crouch, holotype BPI 892770, culture ex-type CBS131599.

Hosts - Sorghastrum nutans (Crouch 2014)

Distribution - USA (Crouch 2014)

Notes - This species claded in the caudatum species complex in Crouch (2014). Colletotrichum somersetense, was isolated as a pathogen, however no pathogenicity data are available to confirm this. Whole genomic data for this species are available (https://mycocosm.jgi.doe.gov/Colletotrichum/Colletotrichum.info.html).

Colletotrichum sublineola Henn., in Kaba't and Buba'k, Fungi Imperfecti Exs. Century II: No. 186 (1904)

Sexual morph not reported. See Crouch \& Tomaso-Peterson (2012) for illustrations and descriptions of asexual morph.

Type - Togo, Burkina Fasso, isolated from Sorghum vulgare, collection date unknown, culture ex-epitype CBS 131301.

Hosts - Sorghum sp. (Crouch \& Tomaso-Peterson 2012)

Distribution - Togo, USA (Crouch \& Tomaso-Peterson 2012)

Notes - This species is recorded only from Sorghum sp. and is a member of graminicola 
clade. It can be distinguished by any of the loci (apn2, ITS, mat and sod2) used in Crouch \& Tomaso-Peterson (2012). Whole genomic data for this species are available (https://mycocosm.jgi.doe.gov/Colletotrichum/Colletotrichum.info.html, Baroncelli et al. 2014a).

Colletotrichum zoysiae J.A. Crouch, IMA Fungus 5(1):27 (2014)

Sexual morph not reported. See Crouch (2014) for illustrations and descriptions of asexual morph.

Type - Japan, Yamaguchi, on leaves of Zoysia tenuifolia, May 1998, A. Tanaka, holotype BPI 884090-holotype, ex-holotype MAFF238573

Hosts - Zoysia tenuifolia (Crouch 2014)

Distribution - Japan (Crouch 2014)

Notes - Colletotrichum zoysiae was isolated as a pathogen. However, no data are available to confirm its pathogenicity. This taxon claded in the caudatum clade and can be identified by any of the gene loci (apn2, ITS, mat and sod2) used in Crouch (2014). Whole genomic data for this species are available (https://mycocosm.jgi.doe.gov/Colletotrichum/Colletotrichum.info.html).

\section{Dematium Species Complex}

The dematium species complex includes $C$. dematium and 17 closely related species. Species of this complex appear to be characteristic of temperate climates (Cannon et al. 2012). The type species of Colletotrichum, C. lineola, belongs in this species complex (Damm et al. 2009). There are two subclades within this complex. Typical characters of this complex are the conidia with an almost straight central part that bent abruptly to the apex and the truncate base, which gives them an almost angular shape (Damm et al. 2009). Some of the species are either host specific or geographically restricted. However, for some of these species only a single strain is available. Therefore, new collections are needed to confirm the host-specificity of the species. A combined multigene analysis of ITS, gapdh, chs-1, act and $t u b 2$ sequence data is given in Fig. 9, Table 8.

Colletotrichum anthrisci Damm, P.F. Cannon \& Crous, in Damm et al., Fungal Diversity 39: 56 (2009)

Sexual morph not reported. See Damm et al. (2009) for illustrations and descriptions of asexual morph.

Type - Netherlands, Utrecht, isolated from dead stems of Anthriscus sylvestris, 12 Sep. 2009, U. Damm, holotype CBS H-20355, culture extype CBS 125334.

Hosts - Anthriscus sylvestris (Damm et al. 2009)

Distribution - Netherlands (Damm et al. 2009)

Notes - Colletotrichum anthrisci is recorded as a saprobe only from Anthriscus sylvestris in the Netherlands. This species differs from other species in this complex in having a constricted base of setae and very long, navicular appressoria (Damm et al. 2009).

Colletotrichum circinans (Berk.) Voglino, Annali R. Accad. Agric. Torino 49: 175 (1907)

Sexual morph not reported. See Damm et al. (2009) for illustrations and descriptions of asexual morph.

Type - Serbia, Novi Sad, on Allium cepa, isolated 1980 by Z. KlocokarSmit, CBS H-20356 (dried culture), culture ex-epitype CBS 221.81.

Hosts - Allium cepa (Damm et al. 2009)

Distribution - Serbia (Damm et al. 2009), temperate regions (Jayawardena et al. 2016a)

Notes - Colletotrichum circinans was originally described from diseased onion bulbs grown from seed originating from the Paris area (Berkeley 1851). Epitypification of the species was carried out in Damm et al. (2009). This taxon is not restricted to a specific country or continent, but appears to be common in temperate regions (Damm et al. 2009, Jayawardena et al. 2016a). This taxon can be distinguished by its dark brown chlamydospores in chains and clades (Damm et al. 2009). 
Colletotrichum dematium (Pers.) Grove, J. Bot., Lond. 56: 341 (1918)

Sexual morph not reported. See Damm et al. (2009) for illustrations and descriptions of asexual morph.

Type - France, from dead leaf of Eryngium campestre, epitype CBS H-20357 (dried culture) culture ex-epitype CBS 125.25.

Hosts - Bidens pilosa, Eryngium campestre, Genista tinctoria, Solanum tuberosum, Vitis vinifera (Damm et al. 2009)

Distribution - Australia, Cuba, Czech Republic, France, South Africa, Venezuela, West Indies (Damm et al. 2009)

Notes - This taxon is recorded as a pathogen, endophyte and a saprobe. This taxon is characterized by the angular shape of its conidia, the production of red pigments in fresh cultures and by its well developed sclerotium-like conidiomata (Damm et al. 2009).

Colletotrichum eryngiicola Jayaward., Bulgakov \& K.D. Hyde, in Buyck et al., Cryptog. Mycol. 38(1): 141 (2017)

Sexual morph not reported. See Buyck et al. (2017) for illustrations and descriptions of asexual morph.

Type - Russia, Rostov Region, Shakhty City, steppe slopes near Grushevsky pond, on dying leafstalks of Eryngium campestre, 18 February 2016, TS Bulgakov, holotype MFU16-1477, culture ex-holotype MFLUCC 17-0318.

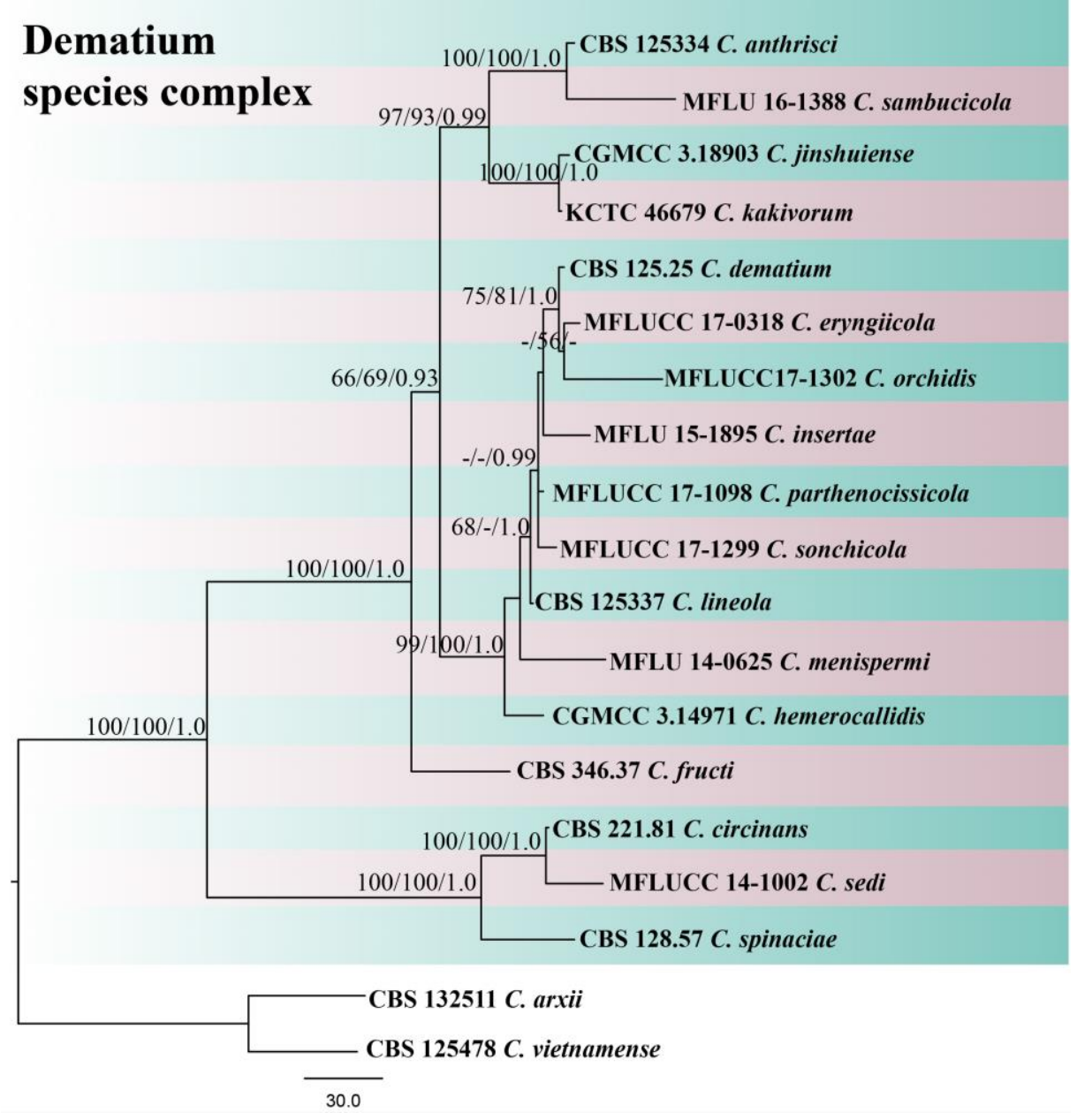

Figure 9 - The dematium complex. The most parsimonious tree obtained from a heuristic search of combined ITS, gapdh, chs-1, act and tub2 sequence data. The MP and ML bootstrap support values $\geq 50 \%$ and $\mathrm{BYPP} \geq 0.90$ are indicated at the nodes and branches. The tree is rooted with $C$. arxii (CBS 132511) and C. vietnamense (CBS 125478). 
Table 8 GenBank accession numbers of species in the dematium complex

\begin{tabular}{|c|c|c|c|c|c|c|c|}
\hline Species name & Type/Reference strain & ITS & gapdh & chs-1 & act & $t u b 2$ & References \\
\hline C. anthrisci & CBS 125334 & GU227845 & GU228237 & GU228335 & GU227943 & GU228139 & Damm et al. (2009) \\
\hline C. circinans & CBS 221.81 & GU227855 & GU228247 & GU228345 & GU227953 & GU228149 & Damm et al. (2009) \\
\hline C. dematium & CBS 125.25 & GU227819 & GU228211 & GU228309 & GU227917 & GU228113 & Damm et al. (2009) \\
\hline C. eryngiicola & MFLUCC 17-0318 & KY792726 & KY792723 & KY792720 & KY792717 & KY792729 & Buyck et al. (2017) \\
\hline C. fructi & CBS 346.37 & GU227844 & GU228236 & GU228334 & GU227942 & GU228138 & Damm et al. (2009) \\
\hline C. hemerocallidis & CGMCC 3.14971 & JQ400005 & JQ400012 & Q399998 & JQ399991 & JQ400019 & Yang et al. (2012a) \\
\hline C. insertae & MFLU 15-1895 & KX618686 & KX618684 & KX618683 & KX618682 & KX618685 & Hyde et al. (2016) \\
\hline C. jinshuiense & CGMCC 3.18903 & MG748077 & MG747995 & MG747913 & MG747767 & MG748157 & Fu et al. (2019) \\
\hline C. kakivorum & КСТС 46679 & LC324781 & LC324787 & LC324783 & LC324785 & LC324791 & Jung \& Lee (2018) \\
\hline C. lineola & CBS 125337 & GU227829 & GU228221 & GU228319 & GU227927 & GU228123 & Damm et al. (2009) \\
\hline C. menispermi & MFLU 14-0625 & KU242357 & KU242356 & KU242355 & KU242353 & KU242354 & Li et al. (2016) \\
\hline C. orchidis & MFLUCC17-1302 & MK502144 & MK496857 & MK496855 & MK496853 & MK496859 & Hyde et al. (2020) \\
\hline C. parthenocissicola & MFLUCC 17-1098 & MK629452 & MK639362 & MK639356 & MK639358 & MK639360 & Yuan et al. (2020) \\
\hline C. quinquefoliae & MFLU 14-0626 & KU236391 & KU236390 & - & KU236389 & KU236392 & Li et al. (2016) \\
\hline C. sambucicola & MFLU 16-1388 & KY098781 & KY098780 & KY098779 & KY098778 & KY098782 & Tibpromma et al. (2017) \\
\hline C. sedi & MFLUCC 14-1002 & KM974758 & KM974755 & KM974754 & KM974756 & KM974757 & Liu et al. (2015) \\
\hline C. sonchicola & MFLUCC 17-1299 & KY962757 & KY962754 & KY962751 & KY962748 & & Jayawardena et al. (2017) \\
\hline C. spinaciae & CBS 128.57 & GU227847 & GU228239 & GU228337 & GU227945 & GU228141 & Damm et al. (2009) \\
\hline
\end{tabular}

Hosts - Eryngium campestre (Buyck et al. 2017)

Distribution - Russia (Buyck et al. 2017)

Notes - Differs from other known species of the dematium complex in having conidiomata with abundant setae, as well as in producing larger conidia $(\mathrm{L} / \mathrm{W}$ ratio $=6.3$ ) (Buyck et al. 2017). This species is only recorded from its type strain.

Colletotrichum fructi (F. Stevans \& J.G. Hall) Sacc. [as 'fructus'], Syll. fung. (Abellini) 22: 1201 (1913)

Sexual morph not reported. See Damm et al. (2009) for illustrations and descriptions of asexual morph.

Type - USA, Rhode Island, Kingston, on fruit of Malus sylvestris, epitype CBS H-20360 (dried culture), culture ex-epitype CBS 346.37.

Hosts - Malus sylvestris, Pyrus malus (Damm et al. 2009)

Distribution - USA (Damm et al. 2009)

Notes - This taxon is described as a pathogen and is very rarely mentioned in the literature. There are no records of this species other than the strains in Damm et al. (2009) and no pathogenicity data are available.

Colletotrichum hemerocallidis Yan L. Yang, Zuo Y. Liu, K.D. Hyde \& L. Cai, Tropical Plant Pathology 37(3): 170 (2012)

Sexual morph not reported. See Yang et al. (2012a) for illustrations and descriptions of asexual morph. 
Type - China, Guizhou Province, Guiyang, on dead stalk of Hemerocallis fulva var. kwanso Regel, 1 July 2008, Y. L. Yang, holotype GZAAS 080059, culture ex-holotype CGMCC 3.14971.

Hosts - Hemerocallis fulva (Yang et al. 2012a)

Distribution - Canada, China (Yang et al. 2012a)

Notes - This taxon is recorded as a saprobe. Colletotrichum hemerocallidis is similar to $C$. anthrisci and $C$. lineola, however differs from its wider conidia and clavate mycelia appressoria (Yang et al. 2012a).

Colletotrichum insertae Jayaward., Bulgakov \& K.D. Hyde, in Hyde et al., Fungal Diversity 80: 176 (2016)

Sexual morph not reported. See Hyde et al. (2016) for illustrations and descriptions of asexual morph.

Type - Russia, Rostov region, Rostov-on-Don city, Botanical garden of Southern Federal University, High Park, dying twigs and leafstalks, on Parthenocissus inserta, 15 April 2015, T. S. Bulgakov (T191), holotype MFLU 15-1895.

Hosts - Parthenocissus inserta (Hyde et al. 2016)

Distribution - Russia (Hyde et al. 2016)

Notes - Colletotrichum insertae is recorded as a saprobe from Parthenocissus inserta. There is no culture available for this species and sequence data were gained from direct DNA sequencing of the fruiting bodies (Hyde et al. 2016).

Colletotrichum jinshuiense M. Fu \& G.P. Wang, in Fu et al., Persoonia 42: 21 (2018) morph.

Sexual morph not reported. See Fu et al. (2019) for illustrations and descriptions of asexual

Type - China, Hubei Province, Wuhan City, on leaves of Pyrus pyrifolia cv. 'Jinshui', 1 Aug. 2016, M. Fu, holotype HMAS 247824, culture ex-type CGMCC 3.18903.

Hosts - Pyrus pyrifolia (Fu et al. 2019)

Distribution - China (Fu et al. 2019)

Notes - Colletotrichum jinshuiense can be distinguished by its gapdh and tub2 sequence data. $\mathrm{Fu}$ et al. (2019) mentioned that this species did not produce asexual and sexual morphs on PDA or SNA. However, they easily developed on pear fruit and leaves. This indicates that pear tissue plays an important part in the epidemiology and life cycle of $C$. jinshuiense. Pathogenicity data are available for this taxon.

Colletotrichum kakiivorum H.Y. Jung \& S.Y. Lee [as 'kakivorum'], in Lee \& Jung, Mycol. Progr. 17(10): 1118 (2018)

Sexual morph not reported. See Lee \& Jung (2018) for illustrations and descriptions of asexual morph.

Type - Korea, Sangju-si, Gyeongbuk province, isolated from diseased leaves showing leaf spot on Diospyrus kaki, holotype KCTC 46679.

Hosts - Diospyrus kaki (Lee \& Jung 2018)

Distribution - Korea (Lee \& Jung 2018)

Notes - This taxon was isolated from leaf spots on diseased Diospyros kaki leaves and pathogenicity study has been carried out to confirm its pathogenicity. According to the phylogenetic analysis, Colletotrichum kakivorum is sister of the clade accommodating $C$. anthrisci and $C$. sambucicola.

Colletotrichum lineola Corda, in Sturm, Deutschlands Flora (Nürnberg) 3: 41 (1831)

Sexual morph not reported. See Damm et al. (2009) for illustrations and descriptions of asexual morph.

Type - Czech Republic, Central Bohemia, Lazne Tousen (ca $25 \mathrm{~km}$ E of Prague), left bank of river Labe, from dead stem of Apiaceae plant, 20 Sep. 2009, M. Reblová, epitype CBS H-20362, 
culture ex-epitype CBS 125337.

Hosts - Anthriscus sp., Apiaceae, Allium giganteum, Astrantia major, Clarkia elegans Euphorbia egula, Heracleum sp., Fragaria sp. (on petioles), Lupinis polyphyllus, Prunus domestica (rotten fruit), Symplocarpus foetidus, Trillium sp., Tussilago farfara (Damm et al. 2009)

Distribution - Canada, Czech Republic, Germany, Netherlands, USA, Zimbabwe (Damm et al. 2009)

Notes - Colletotrichum lineola is the type species of Colletotrichum and seems to be a widespread, primarily temperate species associated with a very wide range of plant species. This taxon is characterized by small, compressed acervuli, emerging in rows/lines (Damm et al. 2009). This species is recognized as a saprobe as well as a pathogen. In Farr \& Rossman (2021) there are 44 records of this species; however, some are based on only morphology. There are no new records of this species after Damm et al. (2009).

Colletotrichum menispermi Chethana, Jayawardena, Bulgakov \& K.D. Hyde, in Li et al., Fungal Diversity 78: 80 (2016)

Sexual morph not reported. See Li et al. (2016) for illustrations and descriptions of asexual morph.

Type - Russia, Rostov region, Rostov-on-Don city, Botanical Garden of Southern Federal University, introductional nursery, on dead twigs of Menispermum dauricum, 5 March 2014, T.S. Bulgakov, holotype MFLU 14-0625.

Hosts - Menispermum dauricum (Li et al. 2016b)

Distribution - Russia (Li et al. 2016b)

Notes - There is no culture available for this saprobic species; hence the sequence data were obtained from direct DNA extraction of fruiting bodies. It differs from its sister taxon $C$. quinquefoliae in having larger conidiomata with minute 2-3-septate setae which cannot be observed by unaided eye (Li et al. 2016b).

Colletotrichum orchidis Jayaward., Camporesi \& K.D. Hyde, in Hyde et al., Mycosphere 11(1): 595 (2020)

Sexual morph not reported. See Hyde et al. (2020) for illustrations and descriptions of asexual morph.

Type - Italy, Province of Forlì-Cesena, near Premilcuore, on living dead aerial stem of Orchis sp., 5 October 2016, E. Camporesi (IT3118), holotype MFLU 16-2551, culture-extype MFLUCC 17-1302.

Hosts - Orchis sp. (Hyde et al. 2020)

Distribution - Italy (Hyde et al. 2020)

Notes - This species is recorded as a saprobe from Orchis sp. It is a sister to C. dematium and differs in having smaller conidia and lower L/W ratio (see Hyde et al. 2020).

Colletotrichum parthenocissicola Jayawardena, Bulgakov, Huanraleuk \& K.D. Hyde, in Yuan et al., Fungal Diversity: 10.1007/s13225-020-00461-7, [88] (2020)

Sexual morph not reported. See Yuan et al. (2020) for illustrations and descriptions of asexual morph.

Type - Russia, Rostov region, Shakhty City, private garden, dying and dead twigs and leafstalks of Parthenocissus quinquefolia, 5 March 2016, Timur S. Bulgakov (T-1263), holotype MFLU 16-1557, culture ex-type MFLUCC 17-1098.

Hosts - Parthenocissus quinquefolia (Yuan et al. 2020)

Distribution - Russia (Yuan et al. 2020)

Notes - This taxon was isolated as a saprobe on Parthenocissus quinquefolia from Russia. It is sister to $C$. insertae and can be distinguished by its longer setae and conidia (Yuan et al. 2020). 
Colletotrichum quinquefoliae Jayawardena, Bulgakov \& K.D. Hyde, in Li et al., Fungal Diversity 78: 83 (2016)

Sexual morph not reported. See Li et al. (2016) for illustrations and descriptions of asexual morph.

Type - Russia, Rostov region, Rostov-on-Don city, Botanical Garden of Southern Federal University, Higher Park, underwood, on Parthenocissus quinquefolia, 5 March 2014, T.S. Bulgakov, holotype MFLU 14-0626.

Hosts - Parthenocissus quinquefolia (Li et al. 2016b)

Distribution - Russia (Li et al. 2016b)

Notes - This taxon was isolated as a saprobe from Russia. A culture is not available for this species, hence direct DNA extraction from fruiting bodies were carried out. This species differs from $C$. menispermi in having larger conidiomata, 1-5-septate, long setae, with a larger base and conidia with an acute base ( $\mathrm{Li}$ et al. 2016b).

Colletotrichum sambucicola Jayaward., Camporesi \& K.D. Hyde, in Tibpromma et al., Fungal Diversity 83: 131 (2017)

Sexual morph not reported. See Tibpromma et al. (2017) for illustrations and descriptions of asexual morph.

Type - Italy, Province of Forlì-Cesena [FC], Meldola, on dead branch of Sambucus ebulus, $30^{\text {th }}$ March 2016, Erio Camporesi (IT 2902), holotype, MFLU 16-2675, ex-type culture, MFLUCC 16-1388.

Hosts - Sambucus ebulus (Tibpromma et al. 2017)

Distribution - Italy (Tibpromma et al. 2017)

Notes - This taxon is sister with $C$. anthrisci and differs in having conidiogenous cells with a distinct collarette, as well as having smaller conidia (Tibpromma et al. 2017).

Colletotrichum sedi Jayaward., Bulgakov \& K.D. Hyde, in Liu et al., Fungal Diversity: 10.1007/s13225-015-0324-y, [27] (2015)

Sexual morph not reported. See Liu et al. (2015b) for illustrations and descriptions of asexual morph.

Type - Russia, Rostov region, Rostov-na-Donu city, Botanical garden of Southern Federal University, flowerbed, on Sedum sp., 05 March 2014, Timur Bulgakov (T94), holotype MFLU 140623, cultures ex-type MFLUCC 14-1002.

Hosts - Sedum sp. (Liu et al. 2015b)

Distribution - Russia (Liu et al. 2015b)

Notes - Colletotrichum sedi clades in the subclade comprising $C$. circinans and $C$. spinaciae within the dematium complex (Liu et al. 2015b). It can be distinguished by having longer setae with 1-4-septate, simple conidiophores, solitary to aggregated appressoria, in small groups or short chains and smaller conidia (Liu et al. 2015b).

Colletotrichum sonchicola Jayaward., Camporesi \& K.D. Hyde, in Jayawardena et al., Phytotaxa 314(1): 58 (2017)

Sexual morph not reported. See Jayawardena et al. (2017) for illustrations and descriptions of asexual morph.

Type - Italy, Province of Forlì-Cesena [FC]: Riofreddo - Bagno di Romagna, on dead and aerial stem of Sonchus sp., 2 October 2016, Erio Camporesi, holotype MFLU 16-2539, ex-type culture MFLUCC 17-1299.

Hosts - Sonchus sp. (Jayawardena et al. 2017)

Distribution - Italy (Jayawardena et al. 2017)

Notes - Colletotrichum sonchicola was isolated as a saprobe. It differs from its sister taxa in having appressoria, short setae and longer conidia (Jayawardena et al. 2017). 
Colletotrichum spinaciae Ellis \& Halst., J. Mycol. 6(1): 34 (1890)

Sexual morph not reported. See Damm et al. (2009) for illustrations and descriptions of asexual morph.

Reference strain - Netherlands, on Spinacia oleracea, isolated Dec. 1957 by G. van den Ende, living culture CBS 128.57.

Hosts - Chenopodium album, Medicago sativa, Portulaca oleracea, Spinacea sp. (Damm et al. 2009)

Distribution - Canada, Germany, Italy, the Netherlands, Turkey, USA (Damm et al. 2009)

Notes - This taxon is a well-known pathogen on Spinach and is closely related to $C$. circinans (Damm et al. 2009).

\section{Dracaenophilum Species Complex}

The dracaenophilum species complex includes $C$. dracaenophilum and five distantly related species. Damm et al. (2019) mentioned that this is not a species complex in a strict sense. All species form cylindrical conidia with round ends, some with sometimes truncate bases or with a basal scar. Except for $C$. tropicicola other species appear to be host-specific. However, for some of these species only a single strain is available (Damm et al. 2019). A combined multigene analysis of ITS, gapdh, chs-1, act and tub2 sequence data is given in Fig. 10, Table 9.

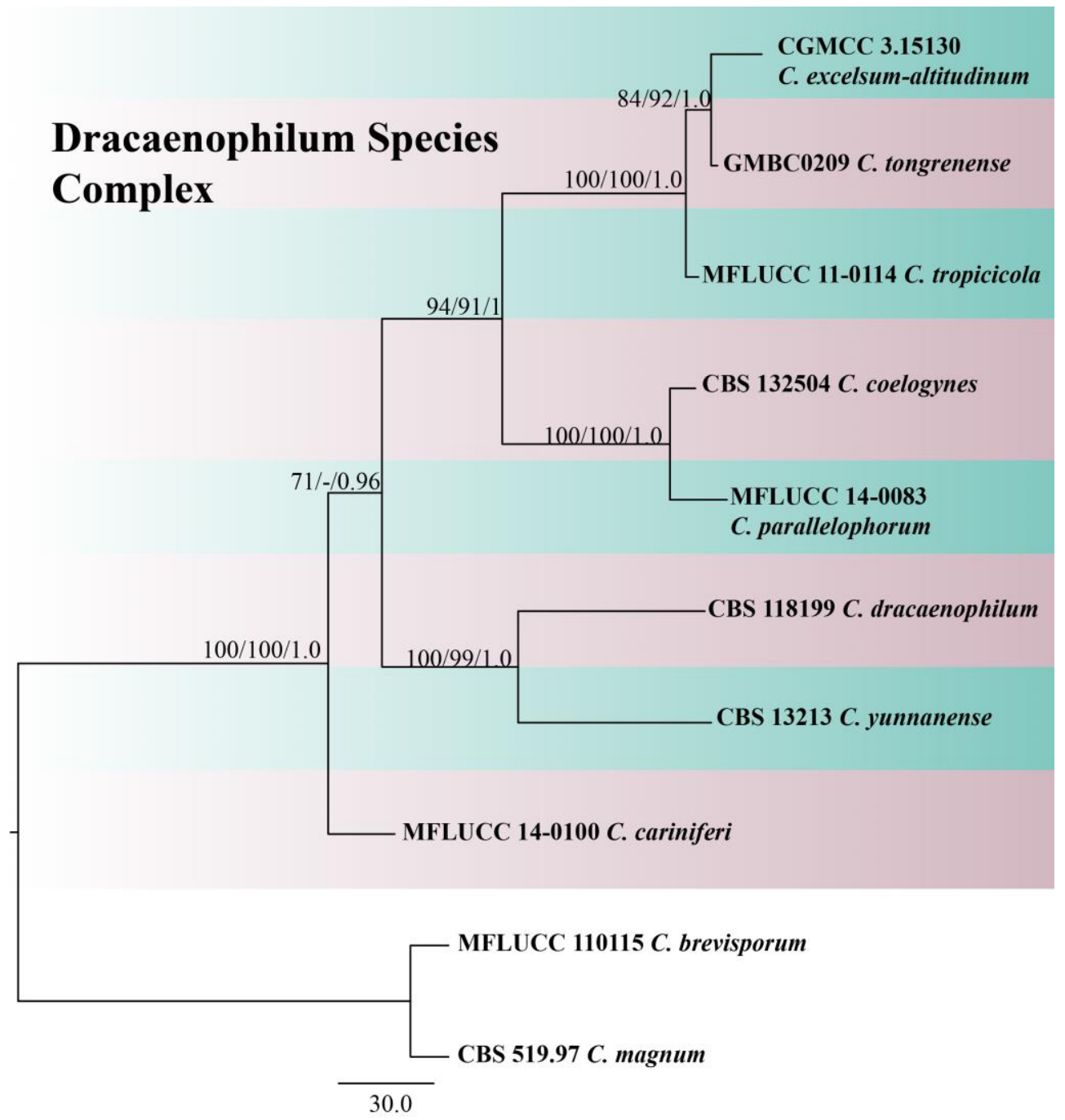

Figure 10 - The dracaenophilum complex. The most parsimonious trees obtained from a heuristic search of combined ITS, gapdh, chs-1, act and tub2 sequence data. The MP and ML bootstrap support values $\geq 50 \%$ and $\mathrm{BYPP} \geq 0.90$ are indicated at the nodes and branches. The tree is rooted with $C$. brevisporum (MFLUCC 1100115) and C. magnum (CBS 519.97). 
Table 9 GenBank accession numbers of species in the dracaenophilum complex

\begin{tabular}{|c|c|c|c|c|c|c|c|}
\hline Species name & Type/Reference strain & ITS & gapdh & chs-1 & act & tub2 & References \\
\hline C. cariniferi & MFLUCC 14-0100 & MF448521 & & & & MH351274 & Ma et al. (2018) \\
\hline C. coelogynes & CBS 132504 & MG600713 & MG600776 & MG600836 & MG600920 & MG600980 & Damm et al. (2019) \\
\hline C. dracaenophilum & CBS 118199 & JX519222 & JX546707 & JX519230 & JX519238 & JX519247 & Farr et al. (2006) \\
\hline C. excelsum-altitudinum & CGMCC 3.15130 & HM751815 & KC843502 & & KC843548 & JX625211 & Damm et al. (2019) \\
\hline C. parallelophorum & MFLUCC 14-0083 & MF448525 & MK165695 & & & MH351280 & Ma et al. (2018) \\
\hline C. tongrenense & GMBC0209 & MH482933 & MH705332 & & MH717074 & MH729805 & Zhou et al. (2019) \\
\hline C. tropicicola & MFLUCC 11-0114 & JN050240 & JN050229 & & JN050218 & JN050246 & Damm et al. (2019) \\
\hline C. yunnanense & CBS 13213 & JX546804 & JX546706 & JX519231 & JX519239 & JX519248 & Damm et al. (2019) \\
\hline
\end{tabular}

Colletotrichum cariniferi X.Y. Ma, K.D. Hyde \& Jayawardena, in Ma et al., MycoKeys 43: 33 (2018)

Sexual morph not reported. See Ma et al. (2018) for illustrations and descriptions of asexual morph.

Type - Thailand, Chiang Rai, Wat Phra That Doi Tung (Temple of Doi Tung Pagodas), Dendrobium cariniferum, 19 December 2013, Sureeporn Nontachaiyapoom, Natdanai Aewsakul and Xiaoya Ma, holotype MFLU 17-1199, culture ex-type MFLUCC 14-0100.

Hosts/substrates - Dendrobium cariniferum (Ma et al. 2018)

Distribution - Thailand (Ma et al. 2018)

Notes - Colletotrichum cariniferi was isolated as an endophyte is closely related to the dracaenophilum species complex (Ma et al. 2018). This species appears to be host specific and restricted to Thailand.

Colletotrichum coelogynes Damm, in Damm et al., Stud. Mycol. 92: 13 (2018)

Sexual morph not reported. See Damm et al. (2019) for illustrations and descriptions of asexual morph.

Type - Germany, Munich, glasshouse, from leaves of Coelogyne sp., 20 Nov. 2010, U. Damm, holotype CBS H-21069, culture ex-holotype CBS 132504.

Hosts - Coelogyne sp. (Damm et al. 2019)

Distribution - Germany (Damm et al. 2019)

Notes - Colletotrichum coelogynes can be identified with all loci (ITS, gapdh, chs-1, act, his3 and tub2) studied in Damm et al. (2019). This taxon has large conidiomata and is the fastest growing species in this complex. Colletotrichum coelogynes seems to be host- specific and restricted to Germany.

Colletotrichum dracaenophilum D.F. Farr \& M.E. Palm, in Farr et al., Mycol. Res. 110(12): 1401 (2006)

Sexual morph not reported. See Damm et al. (2019) for illustrations and descriptions of asexual morph.

Type - China, intercepted in San Francisco, California, USA, from dying stems of Dracaena sanderiana, 25 Sep. 2002, J.R. Nelson, holotype BPI 871498, culture ex-holotype CBS 118199.

Hosts - Dracaena sp. (Damm et al. 2019) 
Distribution - Bulgaria, China (Damm et al. 2019)

Notes - Colletotrichum dracaenophilum can be identified with all loci (ITS, gapdh, chs-1, act, his3 and tub2) studied in Damm et al. (2019). This taxon has the largest conidia in the complex.

Colletotrichum excelsum-altitudinum G. Tao, Zuo Y. Liu \& L. Cai [as 'excelsum-altitudum'], in Tao et al., Fungal Diversity 61: 152 (2013)

Sexual morph not reported. See Tao et al. (2013) for illustrations and descriptions of asexual morph.

Type - CHINA, Guizhou Province, Shuicheng, Baijipo mountain, isolated from healthy leaves of Bletilla ochracea, 28 June 2006, Gang Tao, holotype HMAS244279 (dried culture), culture ex-holotype CGMCC 3.15130.

Hosts - Bletilla ochracea (Tao et al. 2013)

Distribution - China (Tao et al. 2013)

Notes - This species forms shorter conidia than the other species in this complex and can be identified with sequences of all loci available (ITS, gapdh, chs-1, act, his3 and tub2, Damm et al. 2019). Colletotrichum excelsum-altitudinum is only recorded from Bletilla ochracea as an endophyte in China.

Colletotrichum parallelophorum X.Y. Ma, K.D. Hyde \& Jayawardena, in Ma et al., MycoKeys 43: 38 (2018)

See Ma et al. (2018) for illustrations and descriptions of asexual and sexual morphs.

Type - Thailand, Chiang Rai, Wat Phra That Doi Tung, Dendrobium sp., 19 December 2013, Collectors: Sureeporn Nontachaiyapoom, Natdanai Aewsakul, Xiaoya Ma, holotype MFLU 171198, culture ex-holotype MFLUCC 14-0083.

Hosts/substrates - Dendrobium sp. (Ma et al. 2018)

Distribution - Thailand (Ma et al. 2018)

Notes - This species is closely related to $C$. excelsum-altitudum and $C$. tropicicola. Colletotrichum parallelophorum was isolated as an endophyte. This species appears to be host specific and restricted to Thailand.

Colletotrichum tongrenense S.X. Zhou, J.C. Kang \& K.D. Hyde, in Zhou et al., MycoKeys 49: 7 (2019)

Sexual morph not reported. See Damm et al. (2009) for illustrations and descriptions of asexual morph.

Type - China, Guizhou province, Tongren, isolated from healthy stems of Nothapodytes pittosporoides, 27 May 2016, S.X. Zhou and L.J. Qiao, holotype GACP GZU-TRJ1-37 (dried culture), culture ex-type GMBC0209.

Hosts - Nothapodytes pittosporoides (Zhou et al. 2019)

Distribution - China (Zhou et al. 2019)

Notes - This taxon resembles $C$. tropicicola and $C$. excelsum-altitudum. However, C. tongrenense can be distinguished by having longer setae (Zhou et al. 2019). This taxon is an endophyte which appears to be host-specific and has restricted geography in distribution.

Colletotrichum tropicicola Phouliv., Noireung, L. Cai \& K.D. Hyde, Cryptog. Mycol. 33(3): 353 (2012)

Sexual morph not reported. See Noireung et al. (2012) and Damm et al. (2019) for illustrations and descriptions of asexual morph.

Type - Thailand, Chiang Mai Province, Mae Taeng District, Phadeng village, on Citrus maxima, 14 March 2009, Sitthisack Phoulivong, holotype MFLU 110013), culture ex-type MFLUCC 110114. 
Hosts - Citrus maxima (Noireung et al. 2012, Damm et al. 2019), Paphiopedilum bellatulum (Noireung et al. 2012)

Distribution - Thailand (Noireung et al. 2012), Mexico (Damm et al. 2019)

Notes - Colletotrichum tropicicola can be identified with sequences of all loci (ITS, gapdh, act and tub2) available (Noireung et al. 2012). This taxon was isolated from a leaf spot of Citrus maxima in Thailand. However, no pathogenicity data are available for this species.

Colletotrichum yunnanense Xiao Ying Liu \& W.P. Wu, in Liu et al., Mycotaxon 100: 139 (2007)

See Damm et al. (2019) for illustrations and descriptions of asexual and sexual morphs.

Type - China, Yunnan, Kunming Botanical Garden, from healthy leaves of Buxus sp., 5 Nov. 2004, W.P. Wu, holotype WU47182, culture ex-holotype CBS 132135.

Hosts - Buxus sp. (Liu et al. 2007)

Distribution - China (Liu et al. 2007)

Notes - Colletotrichum yunnanense was isolated as an endophyte. This is the only species that the sexual morph has been recorded in dracaenophilum complex (Damm et al. 2019). This taxon can be distinguished from other species of the complex by its smallest appressoria and from any of the loci (ITS, gapdh, chs-1, act, his3 and tub2) used in Damm et al. (2019). Colletotrichum yunnanense appears to be host-specific and restricted to China.

\section{Destructivum Species Complex}

The destructivum species complex is a collection of $C$. destructivum and 16 closely related species that are mainly plant pathogens (Damm et al. 2014). All species in this complex are hemibiotrophic (Damm et al. 2014). O'Connell et al. (2012) showed that the destructivum species complex is monophyletic and distinct from other Colletotrichum species complexes. Species of this complex are characterized by conidia that are slightly curved due to their unilaterally tapering ends and by small inconspicuous acervuli with rather effuse growth (Damm et al. 2014). Some of the species are either host specific or geographically restricted. However, for some of these species only a single strain is available (Damm et al. 2014). A combined multigene analysis of ITS, gapdh, chs-1, act and tub2 sequence data is given in Fig. 11, Table 10. Species are in alphabetical order, hosts and distributions are listed chronologically. According to Damm et al. (2014) all species can be identified by a combination of $t u b 2$ and gapdh sequence data.

Colletotrichum americae-borealis Damm, in Damm et al., Stud. Mycol. 79: 55 (2014)

Sexual morph not reported. See Damm et al. (2014) for illustrations and descriptions of asexual morph.

Type - USA, Utah, Bluffdale (near Salt Lake City), from stems of Medicago sativa, 25 Aug. 2013, U. Damm, holotype CBS H-21661, culture ex-holotype CBS 136232.

Hosts - Medicago sativa (Damm et al. 2014), Glycyrrhiza uralensis (Lyu \& Li 2020)

Distribution - USA (Damm et al. 2014), China (Lyu \& Li 2020)

Notes - This species can be distinguished from other species in this complex by tub2, chs-1, his3 and act sequence data. Colletotrichum americae-borealis was thought to be host-specific in Damm et al. (2014). Lyu \& Li (2020) provided the first report of this species as a pathogen on Glycyrrhiza uralensis.

Colletotrichum antirrhinicola Damm, in Damm et al., Stud. Mycol. 79: 56 (2014)

Sexual morph not reported. See Damm et al. (2014) for illustrations and descriptions of asexual morph.

Type - New Zealand, Auckland, Kingsland, from foliage of Antirrhinum majus, collection date unknown, HM Dance holotype CBS H-21647, culture ex-holotype CBS 102189.

Hosts - Antirrhinum majus (Damm et al. 2014)

Distribution - New Zealand (Damm et al. 2014) 
Notes - Colletotrichum antirrhinicola can be identified by its unique gapdh and ITS sequence data. This taxon is recorded only from the type strain.

Colletotrichum atractylodicola R.J. Zhou \& H.J. Xu, in Xu et al., Mycol. Progr. 17(3): 8 (2017)

Sexual morph not reported. See Xu et al. (2018a) for illustrations and descriptions of asexual morph.

Type - China, Shenyang, Dongling Road, Shenyang Agricultural University, Medicinal Herb Garden, on leaves of Atractylodes chinensis with anthracnose symptoms, 24 Jul. 2013, R.J. Zhou and H.J. Xu, holotype SYAU 130724 (dried leaf), culture ex-type CGMCC3.18761.

Hosts - Atractylodes chinensis (Xu et al. 2018a)

Distribution - China (Xu et al. 2018a)

Notes - Colletotrichum atractylodicola is a confirmed pathogen on Atractylodes chinensis (Xu et al. 2018a). This taxon can be identified by its unique ITS, gapdh, act and tub2 sequence data.

Colletotrichum bryoniicola Damm, in Damm et al., Stud. Mycol. 79 : 57 (2014)

Sexual morph not reported. See Damm et al. (2014) for illustrations and descriptions of asexual morph.

Type - Netherlands, Wissenkerke, Camperduin, from decaying leaves of Bryonia dioica, 27 Aug. 2001, G. Verkley, No. V1114, holotype CBS H-21663, culture ex-holotype CBS 109849.

Hosts - Bryonia dioica (Damm et al. 2014)

Distribution - Netherlands (Damm et al. 2014)

Notes - Colletotrichum bryoniicola can be distinguished by other species of this complex by ITS, gapdh, his 3 and tub2 sequence data (Damm et al. 2014). Conidia of this taxon are broader than that of the other species in the complex.

Colletotrichum destructivum O'Gara, Mycologia 7(1): 38 (1915)

Sexual morph not reported. See Damm et al. (2014) for illustrations and descriptions of asexual morph.

Type - USA, Utah, Salt Lake City, cemetery, from small black spots on petioles of Trifolium hybridum, 24 Aug. 2013, U. Damm, epitype CBS H-21652, culture ex-epitype CBS 136228.

Hosts - genera of Asteraceae, Convolvulaceae, Fabaceae, Magnoliaceae, Menispermaceae, Lamiaceae, Poaceae, Polygonacea and Solanaceae (Damm et al. 2014)

Distribution - Worldwide (Damm et al. 2014, Jayawardena et al. 2016a)

Notes - Colletotrichum destructivum can be distinguished by ITS, his3, act and tub2 sequence data (Damm et al. 2014). Colletotrichum destructivum has been extensively studied as a pathogen.

Colletotrichum fuscum Laubert, Gartenwelt 31: 675. 1927.

Sexual morph not reported. See Damm et al. (2014) for illustrations and descriptions of asexual morph.

Type - Germany, Berlin, garden, from leaf of Digitalis lutea, 2 Aug. 2012, U. Damm, epitype CBS H-21651, culture ex-epitype CBS 133701.

Hosts - Digitalis sp., Heracleum sp. (Damm et al. 2014)

Distribution - Germany, Netherlands (Damm et al. 2014)

Notes - This taxon can be distinguished by its complex appressoria and the ampulliform conidiogenous cells as well as from gapdh sequence data (Damm et al. 2014). Phytotoxin collotin was discovered in one of the strains of C. fuscum by Goodman (1960).

Colletotrichum higginsianum Sacc., J. Agric. Res., Washington 10: 161. 1917.

Sexual morph not reported. See Damm et al. (2014) for illustrations and descriptions of asexual morph. 
Type - Trinidad and Tobago, Trinidad, Wallerfield, from leaf spot on living leaf of Brassica rapa subsp. chinensis, collection date and collector unknown epitype IMI 349061, culture exepitype IMI 349061.

Hosts - genera of Brassicaceae (Damm et al. 2014)

Distribution - Italy, Japan, Korea, Trinidad and Tobago and the USA (Damm et al. 2014)

Notes - There are many records for this species in Farr \& Rossman (2021). As they are based only on morphology, new collections are needed to confirm the associations. This taxon is wellknown and studies as a pathogen on cruciferous plants (O'Donell et al. 2012). Colletotrichum higginsianum can be identified by its ITS and tub2 sequence data from the other species in destructivum complex (Damm et al. 2014). Whole genomic data for this species are available (Zampounis et al. 2016).

Colletotrichum lentis Damm, in Damm et al., Stud. Mycol. 79: 65 (2014)

Sexual morph not reported. See Damm et al. (2014) for illustrations and descriptions of asexual morph.

Type - Canada, Saskatchewan, North Battlefield, from seed, 2001 crop, sample 90812, of Lens culinaris cv. 'CDA Grandora', 2001, R.A.A. Morrall, and Discovery Seed Labs, holotype CBS H-21649, culture ex-holotype CBS 127604.

Hosts - Lens culinaris (Damm et al. 2014), Vicia sativa (Xu \& Li 2015)

Distribution - Canada, Romania (Damm et al. 2014), China (Xu \& Li 2015)

Notes - Colletotrichum lentis is characterised by its slightly curved, fusoid conidia that gradually taper to the acute ends and by the globose appressoria with an entire margin. It can be identified by all loci included in Damm et al. (2014). Hemi-biotrophic nature of this species has been studied (Forseille et al. 2009).

Colletotrichum lini (Westerd.) Tochinai, J. Coll. Agric. Hokkaido Imp. Univ. 14: 176. 1926.

Sexual morph not reported. See Damm et al. (2014) for illustrations and descriptions of asexual morph.

Type - Netherlands, from leaves and stems of Linum sp., collection date and collector unknown, IMI 194722 ex coll. Prof. J. van Westerdijk.

Hosts - Linum sp., Medicago sativa, Nigella sp., Raphanus raphanistrum, Taraxacum sp., Teucrium scorodonia, Trifolium sp. (Damm et al. 2014)

Distribution - France, Germany, Ireland, Netherlands, New Zealand, Tunisia, UK, USA (Damm et al. 2014)

Notes - Colletotrichum lini is distinguishable by $c h s-1$, his3, act and tub2 sequence data (Damm et al. 2014). This species is recorded as a pathogen causing leaf and stem spots of the above mentioned hosts.

Colletotrichum ocimi Damm, in Damm et al., Stud. Mycol. 79: 70 (2014)

Sexual morph not reported. See Damm et al. (2009) for illustrations and descriptions of asexual morph.

Type - Italy, Riviera Ligure, from a black spot on leaf of Ocimum basilicum, collection date and collector unknown, May 1994 by A. Garibaldi, Inst. degli studi di Torino, Depart. di Valorizzazione e Protezione delle Risore agroforestiali, holotype CBS H-21646, culture exholotype CBS 298.94.

Hosts - Ocimum basilicum (Damm et al. 2014, Shivas et al. 2016)

Distribution - Italy (Damm et al. 2014), Australia (Shivas et al. 2016)

Notes - Colletotrichum ocimi forms cylindrical, straight conidia with round ends, resembling species in the gloeosporioides complex. This species can be identified by its unique ITS, chs-1, his3, act and tub2 sequence data (Damm et al. 2014).

Colletotrichum panacicola Uyeda \& S. Takim., in Takimoto, Chosen Nokai-ho 14: 24 (1919) 
Sexual morph not reported. See Damm et al. (2014) for description of asexual morph.

Type - not determined. Reference strain C08087.

Hosts - Panax sp. (Damm et al. 2014)

Distribution - China, Japan, Korea and Russia (Damm et al. 2014)

Notes - This species causes anthracnose on a specific host, however no pathogenicity data are available. Colletotrichum panacicola can be distinguished by ITS and gapdh sequence data (Damm et al. 2014).

Colletotrichum pisicola Damm, in Damm et al., Stud. Mycol. 79: 71 (2014)

Sexual morph not reported. See Damm et al. (2014) for illustrations and descriptions of asexual morph.

Type - USA, Wisconsin, from Pisum sativum, collection date unknown, H.D. van Etten, holotype CBS H-21644, culture ex-holotype CBS 724.97.

Hosts - Pisum sp. (Damm et al. 2014)

Distribution - Ecuador, Mexico, USA (Damm et al. 2014)

Notes - Sequence data of all loci studies by Damm et al. (2014) are unique for this species. Colletotrichum pisicola is characterised by its distinctly curved conidia that gradually taper to towards the acute ends.

Colletotrichum shisoi P. Gan, A. Tsushima, Kawarad., Damm \& K. Shirasu, in Gan et al., Scientific Reports 9 (no. 13349): 2 (2019)

Sexual morph not reported. See Gan et al. (2019) for illustrations and descriptions of asexual morph.

Type - Japan, Osaka, Ibaraki City from lesions of cultivated Perilla frutescens var. crispa cv. Aka-shiso, collection date 1 August 2006, collected by M. Kawaradani, holotype TNS-F-40462, culture ex-holotype JCM 3181.

Hosts - Perilla frutescens (Gan et al. 2019)

Distribution - Japan (Gan et al. 2019)

Notes - This species can be identified by ITS, act, chs-1, gapdh, tub2 sequence data (Gan et al. 2019). Even though this species is recorded as a pathogen, pathogenicity data are unavailable for this species.

Colletotrichum tabacum Böning, Prakt. Bl€att. Pflanzenbau Pflanzenschutz 10: 89. 1932.

Sexual morph not reported. See Damm et al. (2014) for illustrations and descriptions of asexual morph.

Type - France, from Nicotiana tabacum, collection date and collector unknown neotype CBS H-21669, culture ex-neotype CPC18945.

Hosts - Centella asiatica, Nicotiana sp. (Damm et al. 2014)

Distribution - France, India, Germany, Madagascar, Zimbabwe (Damm et al. 2014)

Notes - This taxon can be distinguished by its appressoria with a distinct penetration pore with a dark halo and almost straight long conidia (Damm et al. 2014). Any loci (ITS, gapdh, chs-1, his3, act and tub2) used in Damm et al. (2014) can be used to distinguish this species from the other species of the destructivum complex. Pathogenicity data are unavailable for this species.

Colletotrichum tanaceti Barimani, S.J. Pethybr., Vaghefi, F.S. Hay \& P.W.J. Taylor, P1. Path. 62(6): 1252 (2013)

See Barimani et al. (2013) for illustrations and descriptions of sexual morph. See Damm et al. (2014) for illustrations and descriptions of asexual morph.

Type - Australia, northern Tasmania, Scottsdale, from anthracnose on leaves of Tanacetum cinerariifolium, Aug. 2010, S.J. Pethybridge, culture ex-holotype CBS 132693.

Hosts - Tanacetum cinerariifolium (Damm et al. 2014)

Distribution - Australia, Tasmania (Damm et al. 2014) 
Notes - Colletotrichum tanaceti has distinctly curved conidia (Damm et al. 2014). This taxon formed perithecia in a mating experiment and is heterothallic (Barimani et al. 2013). Intracellular hemibiotrophic infection strategy is suggested for this species (Barimani et al. 2013).

Colletotrichum utrechtense Damm, in Damm et al., Stud. Mycol. $79: 77$ (2014)

Sexual morph not reported. See Damm et al. (2009) for illustrations and descriptions of asexual morph.

Type - Netherlands, Utrecht, from a leaf of Trifolium pratense, 13 Jun. 2011, U. Damm, holotype CBS H-21662, culture ex-holotype CBS 130243.

Hosts - Trifolium pratense (Damm et al. 2014)

Distribution - Netherlands (Damm et al. 2014)

Notes - This species can be distinguished by its unique chs-1, his3 and tub2 sequence data (Damm et al. 2014). This species appears to be host-specific and restricted to Netherlands.

Colletotrichum vignae Damm, in Damm et al., Stud. Mycol. 79: 78 (2014)

Sexual morph not reported. See Damm et al. (2009) for illustrations and descriptions of asexual morph.

Type - Nigeria, from Vigna unguiculata, collection date unknown, R.A. Skipp, holotype CBS H-21648, culture ex-type CBS 501.97.

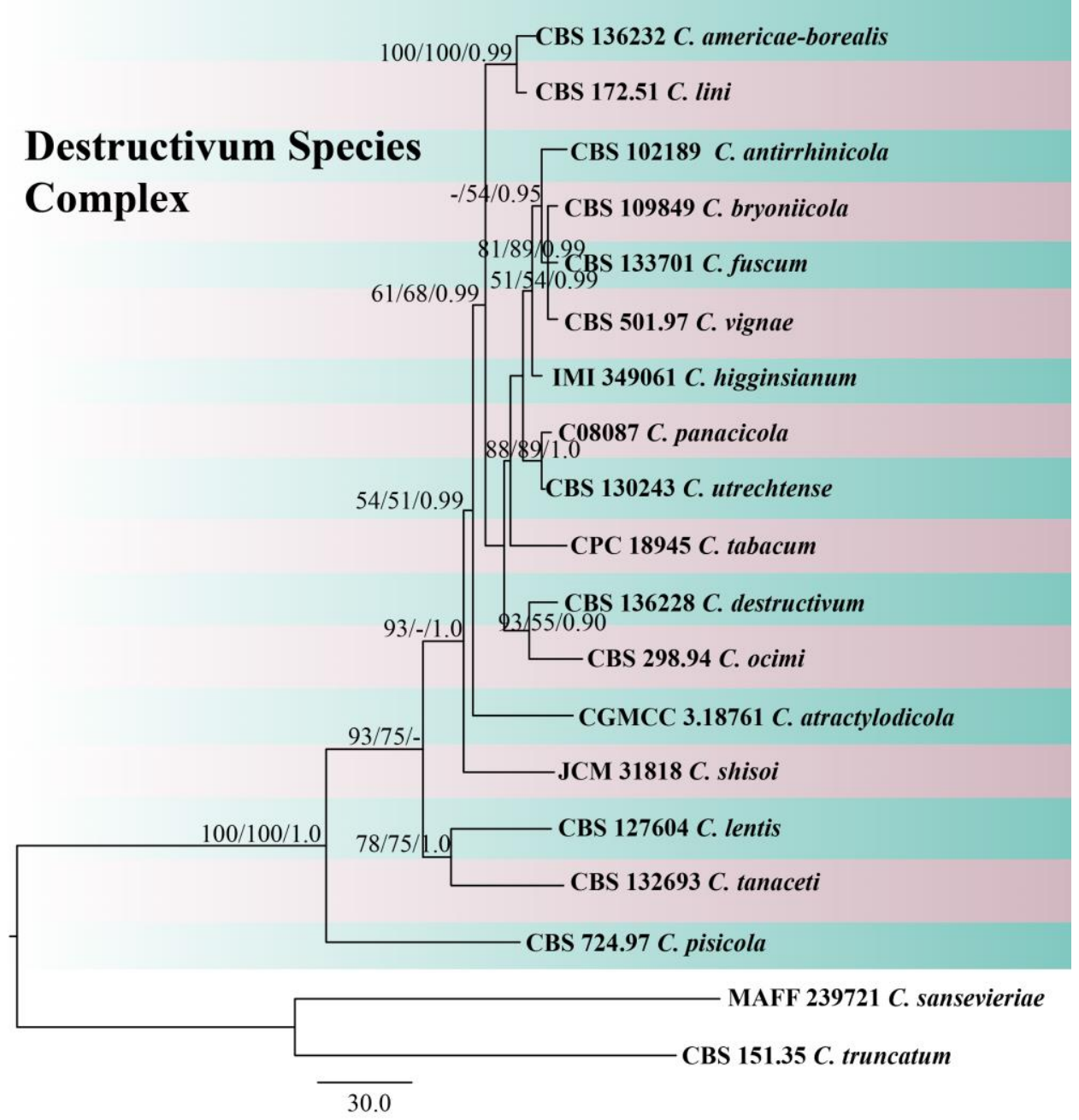

Figure 11 - The destructivum complex. One of the four most parsimonious trees obtained from a heuristic search of combined ITS, gapdh, chs-1, act and tub2 sequence data. The MP and ML bootstrap support values $\geq 50 \%$ and $\mathrm{BYPP} \geq 0.90$ are indicated at the nodes and branches. The tree is rooted with $C$. sansevieriae (MAFF 239721) and C. truncatum (CBS 151.35). 
Table 10 GenBank accession numbers of species in the destructivum complex

\begin{tabular}{|c|c|c|c|c|c|c|c|}
\hline Species name & Type/Reference strain & ITS & gapdh & chs-1 & act & tub2 & References \\
\hline C. americae-borealis & CBS 136232 & KM105224 & KM105579 & KM105294 & KM105434 & KM105504 & Damm et al. (2014) \\
\hline C. antirrhinicola & CBS 102189 & KM105180 & KM105531 & KM105250 & KM105390 & KM105460 & Damm et al. (2014) \\
\hline C. atractylodicola & CGMCC 3.18761 & KR149280 & KR259334 & KR259333 & KR132243 & KU058178 & Xu et al. (2018a) \\
\hline C. bryoniicola & CBS 109849 & KM105181 & KM105532 & KM105251 & KM105391 & KM105461 & Damm et al. (2014) \\
\hline C. destructivum & CBS 136228 & KM105207 & KM105561 & KM105277 & KM105417 & KM105487 & Damm et al. (2014) \\
\hline C. fuscum & CBS 133701 & KM105174 & KM105524 & KM105244 & KM105384 & KM105454 & Damm et al. (2014) \\
\hline C. higginsianum & IMI 349061 & KM105184 & KM105535 & KM105254 & KM105394 & KM105464 & Damm et al. (2014) \\
\hline C. lentis & CBS 127604 & JQ005766 & KM105597 & JQ005787 & JQ005829 & JQ005850 & Damm et al. (2014) \\
\hline C. lini & CBS 172.51 & JQ005765 & KM105581 & JQ005786 & JQ005828 & JQ005849 & Damm et al. (2014) \\
\hline C. ocimi & CBS 298.94 & KM105222 & KM105577 & KM105292 & KM105432 & KM105502 & Damm et al. (2014) \\
\hline C. panacicola & C08087 & GU935869 & GU935849 & & GU944758 & GU935889 & Damm et al. (2014) \\
\hline C. pisicola & CBS 724.97 & KM105172 & KM105522 & KM105242 & KM105382 & KM105452 & Damm et al. 2014 \\
\hline C. shisoi & JCM 31818 & MH660930 & MH660931 & MH660929 & MH660928 & MH660932 & Gan et al. (2019) \\
\hline C. tabacum & CPC 18945 & KM105204 & KM105557 & KM105274 & KM105344 & KM105484 & Damm et al. (2014) \\
\hline C. tanaceti & CBS 132693 & JX218228 & JX218243 & JX259268 & JX218238 & JX218233 & Damm et al. (2014) \\
\hline C. utrechtense & CBS 130243 & KM105201 & KM105554 & KM105271 & KM105411 & KM105481 & Damm et al. (2014) \\
\hline C. vignae & CBS 501.97 & KM105183 & KM105534 & KM105253 & KM105393 & KM105463 & Damm et al. (2014) \\
\hline
\end{tabular}

Hosts - Vigna unguiculata (Damm et al. 2014)

Distribution - Nigeria (Damm et al. 2014)

Notes - This species can be identified by its ITS, gapdh, his3 and act sequence data (Damm et al. 2014). Colletotrichum vignae is recorded only from its type strain.

\section{Gigasporum species complex}

The gigasporum species complex consists of $C$. gigasporum and seven closely related species and is characterised by the formation of large conidia $(>20 \mu \mathrm{m}$ ) (Liu et al. 2014). Species of this complex can be either pathogens or endophytes. Some of the species are either host specific or geographically restricted. However, for some of these species, only a single strain is available, and sometimes the host and the location are unknown (Liu et al. 2014). Therefore, new collections are needed to confirm the host-specificity of the species. A combined multigene analysis of ITS, gapdh, chs-1, act and tub2 sequence data is given in Fig. 12, Table 11. All species within this complex can be identified by any of these five genes (Liu et al. 2014).

Colletotrichum arxii F. Liu, L. Cai, Crous \& Damm, Persoonia, Mol. Phyl. Evol. Fungi 33: 87 (2014)

Sexual morph not reported. See Liu et al. (2014) for illustrations and descriptions of asexual morph.

Type - Berlin, glasshouse, on living leaves of Paphiopedilum sp., Dec. 2010, U. Damm, holotype CBS H-21492, culture ex-type CBS 132511. 
Hosts - Oncidium excavatum, Paphiopedilum sp. (Liu et al. 2014), Vanilla planifolia (Charron et al. 2018)

Distribution - Germany, Netherlands (Liu et al. 2014), Reunion (Charron et al. 2018)

Notes - In the study of Liu et al. (2014), this was isolated as an endophyte. Charron et al. (2018) isolated $C$. arxii from black leaf spot of Vanilla planifolia. The pathogenicity studies showed this species is not pathogenic. Sequence data of ITS and $t u b 2$ can be used to differentiate this taxon from other taxa in the complex.

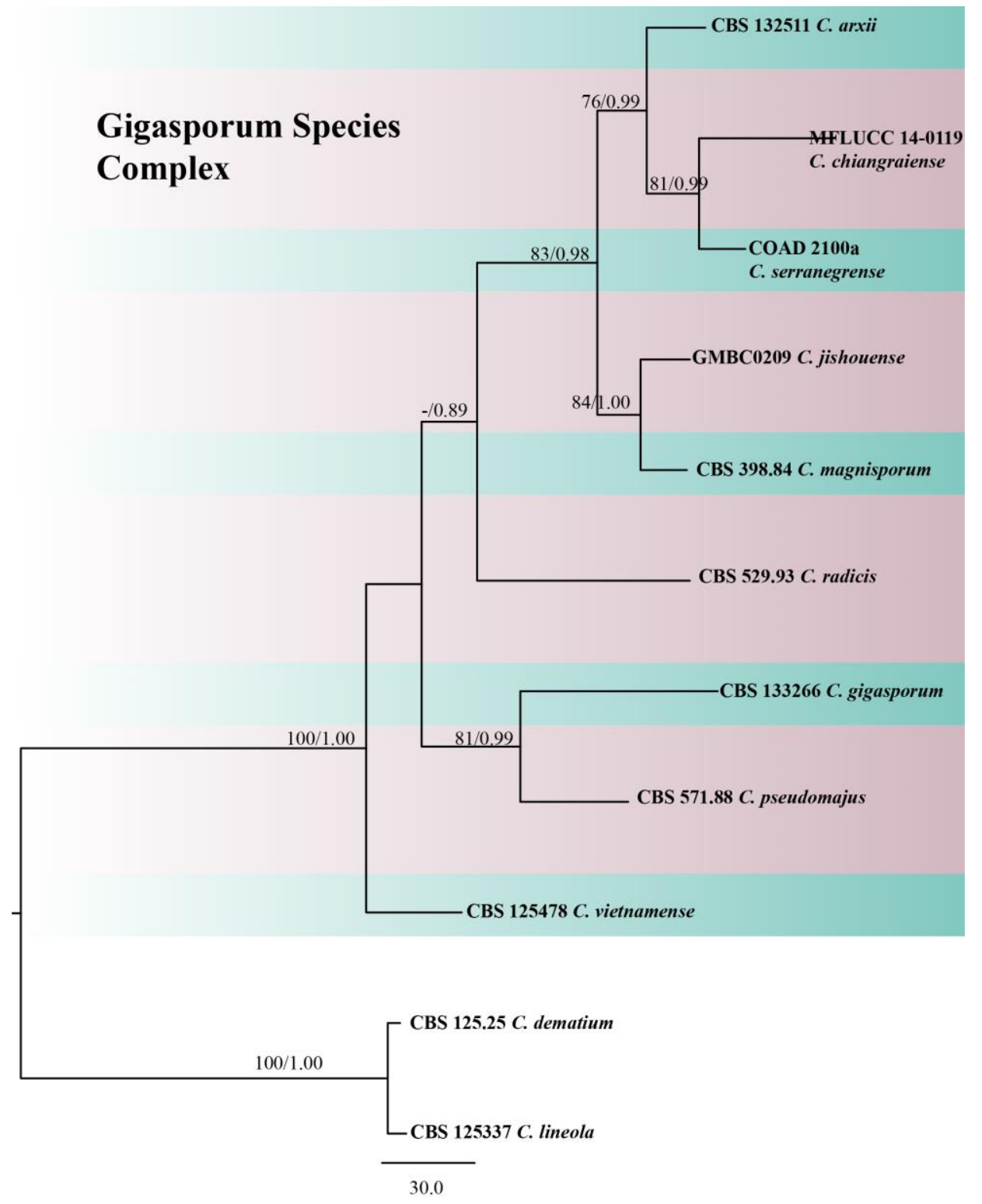

Figure 12 The gigasporum complex. The most parsimonious trees obtained from a heuristic search of combined ITS, gapdh, chs-1, act and tub2 sequence data. The MP bootstrap support values $\geq 50 \%$ and $\mathrm{BYPP} \geq 0.90$ are indicated at the nodes and branches. The tree is rooted with $C$. dematium (CBS 125.75) and C. lineola (CBS 125337).

Colletotrichum gigasporum E.F. Rakotoniriana \& F. Munaut, in Rakotoniriana et al., Mycol. Progr. 12(2): 407 (2013)

See Rakotoniriana et al. (2013) for illustrations and descriptions of asexual and sexual morphs.

Type - Madagascar, Mangoro. Isolated from Centella asiatica, Mai 2003, E.F. Rakotoniriana, holotype MUCL 44947-H (dried culture), culture ex-holotype MUCL 44987. 
Table 11 GenBank accession numbers of species in the gigasporum complex

\begin{tabular}{|c|c|c|c|c|c|c|c|}
\hline Species name & Type/Reference strain & ITS & gapdh & chs -1 & act & tub2 & References \\
\hline C. arxii & CBS 132511 & KF687716 & KF687843 & KF687780 & KF687802 & KF687881 & Liu et al. (2014) \\
\hline C. gigasporum & CBS 133266 & KF687715 & KF687822 & KF687761 & & KF687866 & Liu et al. (2014) \\
\hline C. jishouense & GMBC0209 & MH482929 & MH681658 & & MH708135 & MH727473 & Zhou et al. (2019) \\
\hline C. magnisporum & CBS 398.84 & KF687718 & KF687842 & KF687782 & KF687803 & KF687882 & Liu et al. (2014) \\
\hline C. pseudomajus & CBS 571.88 & KF687722 & KF687826 & KF687779 & KF687801 & KF687883 & Liu et al. (2014) \\
\hline C. radicis & CBS 529.93 & KF687719 & KF687825 & KF687762 & KF687785 & KF687869 & Liu et al. (2014) \\
\hline C. serranegrense & COAD 2100a & KY400111 & & KY407894 & KY407892 & KY407896 & Silva et al. (2018) \\
\hline C. vietnamense & CBS 125478 & KF687721 & KF687832 & KF687769 & KF687792 & KF687877 & Liu et al. (2014) \\
\hline
\end{tabular}

Hosts - Camelia sinensis (Alizadeh et al. 2015), Acacia auriculiformis, Centella asiatica, Coffea sp., Diospyros kaki, Homo sapiense, Musa sp., Persea americana, Solanum betaceum, Theobroma cacao, Trichilia tuberculata, Virola surinamensis (Liu et al. 2014, Jayawardena et al. 2016a), Dalbergia odorifera (Wan et al. 2018), Annona sp. (Costa et al. 2019), Platostoma palustre (Hsieh et al. 2020)

Distribution - Iran (Alizadeh et al. 2015), Japan, Madagascar, New Zealand, Panama, Sri Lanka, Thailand, Vietnam (Jayawardena et al. 2016a), Mexico (Cristobal-Martinez et al. 2017), China (Wan et al. 2018), Brazil (Costa et al. 2019), Taiwan (Hsieh et al. 2020)

Notes - Rakotoniriana et al. (2013) introduced this species based on larger conidia and ITS, tub2 sequence data. Colletotrichum gigasporum is not only a plant pathogen, but also a pathogen on humans causing phaeohyphomycotic cysts (Liu et al. 2014). This taxon can be distinguished by any of the loci (ITS, gapdh, chs-1 and tub2) used in Liu et al. (2014). Pathogenicity data are available for this species.

Colletotrichum jishouense S.X. Zhou, J.C. Kang \& K.D. Hyde, in Zhou et al., MycoKeys 49: 7 (2019)

Sexual morph not reported. See Zhou et al. (2019) for illustrations and descriptions of asexual morph.

Type - China, Hunan Province, Jishou City, isolated from healthy roots of Nothapodytes pittosporoides, 27 May 2016, S.X. Zhou, holotype GACP GZU-HJ2-G3 (dried culture), culture ex-type GMBC0209.

Hosts - Nothapodytes pittosporoides (Zhou et al. 2019)

Distribution - China (Zhou et al. 2019)

Notes - Colletotrichum jishouense is a root endophyte. This species has shorter and narrower conidiogenous cells and conidia than all the related species in the gigasporum complex (Zhou et al. 2019). It can be phylogenetically separated by ITS, tub2, act and gapdh sequence data.

Colletotrichum magnisporum F. Liu, L. Cai, Crous \& Damm, Persoonia, Mol. Phyl. Evol. Fungi 33: 91 (2014)

Sexual morph not reported. See Liu et al. (2014) for illustrations and descriptions of asexual morph.

Type - Unknown collection details, holotype CBS H-21491, culture ex-type CBS 398.84.

Hosts - Unknown

Distribution - Unknown

Notes - Colletotrichum magnisporum was introduced based on a single strain and no data are available for its host or location. More collections 
are needed to understand the host association of this species. This taxon can be separated from its closely related taxon $C$. arxii by any of the loci (ITS, gapdh, chs-1 and tub2) used in Liu et al. (2014).

Colletotrichum pseudomajus F. Liu, L. Cai, Crous \& Damm, Persoonia, Mol. Phyl. Evol. Fungi 33: 91 (2014)

See Liu et al. (2014) for illustrations and descriptions of asexual and sexual morphs.

Type - Taiwan, on twig of Camellia sinensis, unknown collection date and collector, holotype CBS H-21493, culture ex-type CBS 571.88.

Hosts - Camellia sinensis (Liu et al. 2014)

Distribution - Taiwan (Liu et al. 2014)

Notes - This species is introduced with a single strain. Colletotrichum pseudomajus can be differentiated by gapdh and tub2 sequence data.

Colletotrichum radicis F. Liu, L. Cai, Crous \& Damm, Persoonia, Mol. Phyl. Evol. Fungi 33: 93 (2014)

Sexual morph not reported. See Liu et al. (2014) for illustrations and descriptions of asexual morph.

Type - Costa Rica, La Selva, host plant unknown (isolated from a plant root), unknown collection date and collector, holotype CBS H-21494, culture ex-type CBS 529.93.

Hosts - unknown (Liu et al. 2014)

Distribution - Costa Rica (Liu et al. 2014)

Notes - Colletotrichum radicis was introduced based on a single strain isolated from an undetermined plant root. This taxon can be differentiated by its closely related taxon $C$. magnisporum by its shorter conidia and any of the loci (ITS, gapdh, chs-1 and tub2) used in Liu et al. (2014).

Colletotrichum serranegrense Meir. Silva \& M.C.M. Kasuya, in da Silva et al., Phytotaxa 351(2): 167 (2018)

Sexual morph not reported. See Silva et al. (2018) for illustrations and descriptions of asexual morph.

Type - Brazil, Minas Gerais: Parque Estadual da Serra Negra, from the roots of Cattleya jongheana, 14 November 2015, Miranda, L., holotype VIC45136, culture ex-type COAD2100.

Hosts - Cattleya jongheana (Silva et al. 2018)

Distribution - Brazil (Silva et al. 2018)

Notes - This taxon was isolated as an endophyte and can be distinguished by any of the loci (ITS, chs-1, act and tub2) used in Silva et al. (2018).

Colletotrichum vietnamense F. Liu, L. Cai, Crous \& Damm, Persoonia, Mol. Phyl. Evol.Fungi 33: 93 (2014)

Sexual morph not reported. See Liu et al. (2014) for illustrations and descriptions of asexual morph.

Type - Vietnam, Lam Dong Province, Dalat, from anthracnose on leaf of Coffea sp., unknown collection date, P. Nguyen \& E. Lijeroth, holotype CBS H-21512, culture ex-type CBS 125478.

Hosts - Coffea sp. (Liu et al. 2014)

Distribution - Vietnam (Liu et al. 2014)

Notes - Colletotrichum vietnamense can be identified by any of the loci (ITS, gapdh, chs-1 and tub2) used in Liu et al. (2014).

\section{Gloeosporioides Species Complex}

The gloeosporioides species complex consists of $C$. gloeosporioides and 51 closely related 
species. This species complex mainly consists of plant pathogens (Weir et al. 2012), but some species were isolated as endophytes (Liu et al. 2015a). Conidia of this species complex are cylindrical with rounded ends tapering slightly towards the base (Weir et al. 2012). Based on the multigene phylogeny, Weir et al. (2012) recognized two subclades within the species complex, namely kahawae and musae (Fig. 13). A combination of apmat and gs sequences can be used to distinguish the species within this complex (Liu et al. 2015a). A combined multigene analysis of ITS, gapdh, chs-1, act and tub2 sequence data is given in Fig. 13, Table 12.

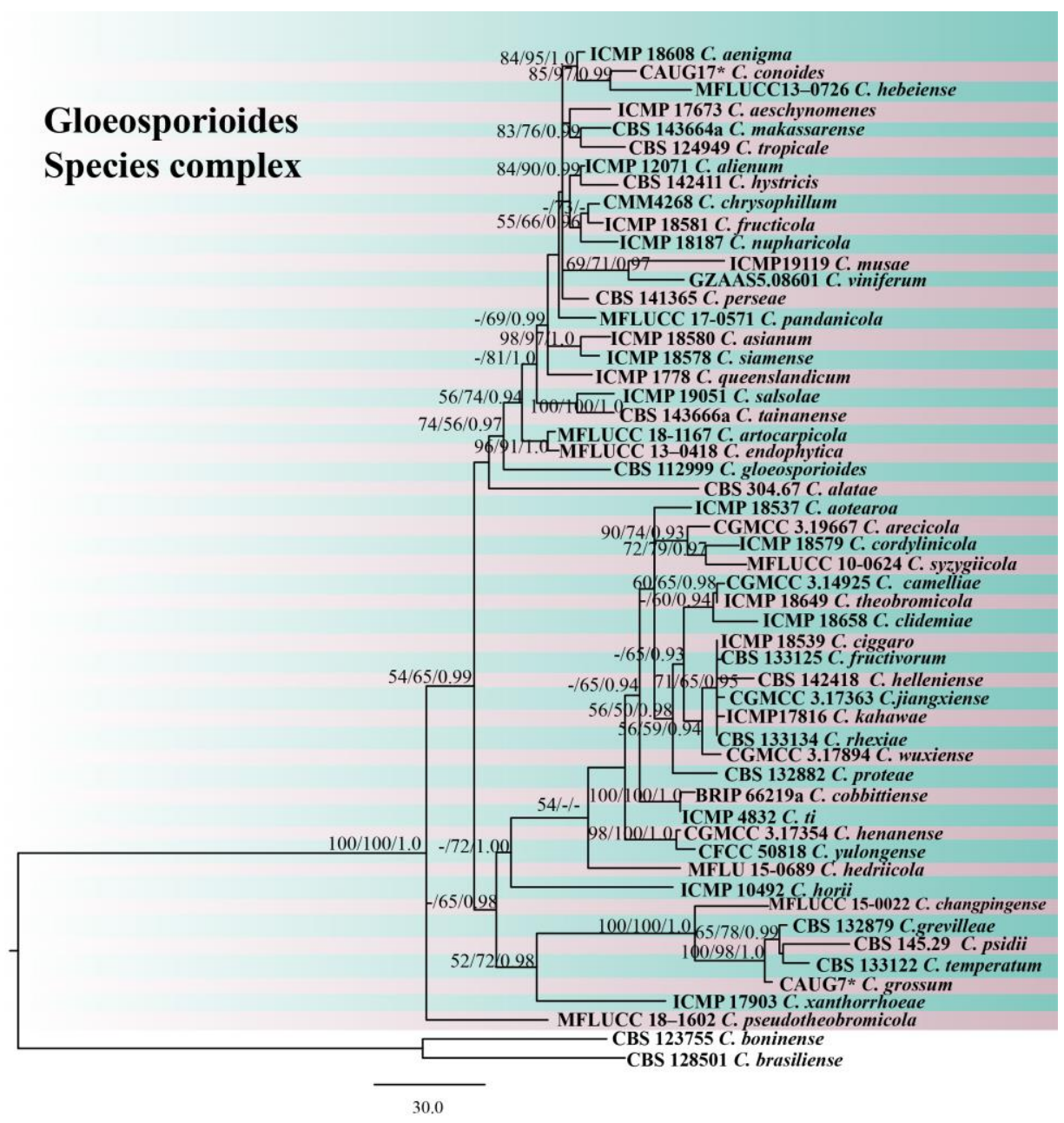

Figure 13 - The gloeosporioides complex. One of the 10 most parsimonious trees obtained from a heuristic search of combined ITS, gapdh, chs-1, act and tub2 sequence data. The MP and ML bootstrap support values $\geq 50 \%$ and $\mathrm{BYPP} \geq 0.90$ are indicated at the nodes and branches. The tree is rooted with $C$. boninense (CBS 123755) and $C$. brasiliense (CBS 128501).

Colletotrichum aenigma B. Weir \& P.R. Johnst., Stud. Mycol. 73: 135 (2012)

See Fu et al. (2019) for illustrations and descriptions of sexual morph. See Weir et al. (2012) for illustrations and descriptions of asexual morph.

Type - Israel, on Persea americana, S. Freeman Avo-37-4B, holotype PDD 102233, culture ex-holotype ICMP 18608.

Hosts - Persea americana, Pyrus pyrifolia (Weir et al. 2012), Hylocereus undatus (Meetum et al. 2015), Vigna unguiculata (Alizadeh et al. 2015), Vitis vinifera (Yan et al. 2015), Sedum kamtschaticum (Choi et al. 2017), Actinidia argute (Wang et al. 2019), Camellia sp. (Chen et al. 2019b, Yang et al. 2019b, Wang et al. 2020)

Distribution - Israel, Japan (Weir et al. 2012), China (Yan et al. 2015, Chen et al. 2019b, Wang et al. 2019, 2020, Yang et al. 2019b), Iran (Alizadeh et al. 2015), Thailand (Meetum et al. 2015), Korea (Choi et al. 2017). 
Notes - Colletotrichum aenigma can be distinguished with the use of TUB2 or GS gene sequence data (Weir et al. 2012). This taxon has a distinctive appearance in culture with sparse, pale aerial mycelium and lacking differentiated acervuli. Pathogenicity studies for this species are available.

Table 12 GenBank accession numbers of species in the gloeosporioides complex

\begin{tabular}{|c|c|c|c|c|c|c|c|}
\hline Species name & Type/Reference strain & ITS & gapdh & chs-1 & act & tub2 & References \\
\hline C. aenigma & ICMP 18608 & JX010244 & JX010044 & JX009774 & JX009443 & JX010389 & Weir et al. (2012) \\
\hline C. aeschynomenes & ICMP 17673 & JX010176 & JX009930 & JX009799 & JX009483 & JX010392 & Weir et al. (2012) \\
\hline C. alatae & CBS 304.67 & JX010190 & JX009990 & JX009837 & JX009471 & JX010383 & Weir et al. (2012) \\
\hline C. alienum & ICMP 12071 & JX010251 & JX010028 & JX009882 & JX009572 & JX010411 & Weir et al. (2012) \\
\hline C. aotearoa & ICMP 18537 & JX010205 & JX010005 & JX009853 & JX009854 & JX010420 & Weir et al. (2012) \\
\hline C. arecicola & CGMCC 3.19667 & MK914635 & MK945455 & MK935541 & MK935374 & MK935498 & Cao et al. (2020) \\
\hline C. artocarpicola & MFLUCC 18-1167 & MN415991 & MN435568 & MN435569 & MN435570 & MN435567 & Bhunjun et al. (2019) \\
\hline C. asianum & ICMP 18580 & JX010196 & JX010053 & JX009867 & JX009584 & JX010406 & Weir et al. (2012) \\
\hline C. camelliae & CGMCC:3.14925 & KJ955081 & KJ954782 & & KJ954363 & KJ955230 & Liu et al. (2015) \\
\hline C. changpingense & MFLUCC 15-0022 & KP683152 & KP852469 & KP852449 & KP683093 & KP852490 & Jayawardena et al. (2016) \\
\hline C. chrysophillum & CMM4268 & KX094252 & KX094183 & KX094083 & KX093982 & KX094285 & Vieira et al. (2017) \\
\hline C. ciggaro & ICMP 18539 & JX010230 & JX009966 & JX009800 & JX009523 & JX010434 & Weir et al. (2012) \\
\hline C. clidemiae & ICMP 18658 & JX010265 & JX009989 & JX009877 & JX009537 & JX010438 & Weir et al. (2012) \\
\hline C. cobbittiense & BRIP 66219a & MH087016 & MH094133 & MH094135 & MH094134 & MH094137 & Crous et al. (2018) \\
\hline C.conoides & CAUG17* & KP890168 & KP890162 & KP890156 & KP890144 & KP890174 & Diao et al. (2017) \\
\hline C. cordylinicola & ICMP 18579 & JX010226 & JX009975 & JX009864 & HM470234 & JX010440 & Phoulivong et al. (2010) \\
\hline C. endophytica & MFLUCC 13-0418 & KC633854 & KC832854 & & KF306258 & & Manamgoda et al. (2013) \\
\hline C. fructicola & ICMP 18581 & JX010165 & JX010033 & JX009866 & FJ907426 & JX010405 & Prihastuti et al. (2009) \\
\hline C. fructivorum & CBS 133125 & JX145145 & & & & JX145196 & Doyle et al. (2013) \\
\hline C. gloeosporioides & CBS 112999 & JQ005152 & JQ005239 & JQ005326 & JQ005500 & JQ005587 & Weir et al. (2012) \\
\hline C.grevilleae & CBS 132879 & KC297078 & KC297010 & KC296987 & KC296941 & KC297102 & Liu et al. (2013) \\
\hline C. grossum & CAUG7* & KP890165 & KP890159 & KP890153 & KP890141 & KP890171 & Diao et al. (2017) \\
\hline C. hebeiense & MFLUCC13-0726 & KF156863 & KF377495 & KF289008 & KF377532 & KF288975 & Yan et al. (2015) \\
\hline C. hederiicola & MFLU 15-0689 & MN631384 & & MN635794 & MN635795 & & Hyde et al. (2020) \\
\hline C. helleniense & CBS 142418 & KY856446 & KY856270 & KY856186 & KY856019 & KY856528 & Guarnaccia et al. (2017) \\
\hline C. henanense & CGMCC 3.17354 & KJ955109 & KJ954810 & & KM023257 & KJ955257 & Liu et al. (2015) \\
\hline C. horii & ICMP 10492 & GQ329690 & GQ329681 & JX009752 & JX009438 & $\mathrm{JX} 010450$ & Weir \& Johnston (2010) \\
\hline C. hystricis & CBS 142411 & KY856450 & KY856274 & KY856190 & KY856023 & KY856532 & Guarnaccia et al. (2017) \\
\hline C.jiangxiense & CGMCC 3.17363 & KJ955201 & KJ954902 & & KJ954471 & KJ955348 & Liu et al. (2013b) \\
\hline C. kahawae & ICMP17816 & JX010231 & JX010012 & JX009813 & JX009452 & JX010444 & Weir et al. (2012) \\
\hline C. makassarense & CBS 143664a, & MH728812 & MH728820 & MH805850 & MH781480 & MH846563 & de Silva et al. (2019) \\
\hline C. musae & ICMP19119 & HQ596292 & HQ596299 & JX009896 & HQ596284 & HQ596280 & Su et al. (2011) \\
\hline C. nupharicola & ICMP 18187 & JX010187 & JX009972 & JX009835 & JX009437 & JX010398 & Weir et al. (2012) \\
\hline
\end{tabular}


Table 12 Continued.

\begin{tabular}{|c|c|c|c|c|c|c|c|}
\hline Species name & Type/Reference strain & ITS & gapdh & chs-1 & act & $t u b 2$ & References \\
\hline C. pandanicola & MFLUCC 17-0571 & MG646967 & MG646934 & MG646931 & MG646938 & MG646926 & Tibpromma et al. (2018) \\
\hline C. perseae & CBS 141365 & KX620308 & KX620242 & & KX620145 & KX620341 & Sharma et al. (2017) \\
\hline C. pseudotheobromicola & MFLUCC 18-1602 & MH817395 & MH853675 & MH853678 & MH853681 & MH853684 & Chethana et al. (2019) \\
\hline C. psidii & CBS 145.29 & JX010219 & JX009967 & JX009901 & JX009515 & JX010443 & Weir et al. (2012) \\
\hline C. proteae & CBS 132882 & KC297079 & KC297009 & KC296986 & KC296940 & KC297101 & Liu et al. (2013) \\
\hline C. queenslandicum & ICMP 1778 & JX010276 & JX009934 & JX009899 & JX009447 & JX010414 & Weir et al. (2012) \\
\hline C. rhexiae & CBS 133134 & JX145128 & & & & JX145179 & Doyle et al. (2013) \\
\hline C. salsolae & ICMP 19051 & JX010242 & JX009916 & JX009863 & JX009562 & JX010403 & Weir et al. (2012) \\
\hline C. siamense & ICMP 18578 & FJ972613 & FJ972575 & JX009865 & FJ907423 & FJ907438 & Prihastuti et al. (2009) \\
\hline C. syzygiicola & MFLUCC 10-0624 & KF242094 & KF242156 & & KF157801 & KF254880 & Udayanga et al. (2013) \\
\hline C. tainanense & CBS 143666a & MH728818 & MH728823 & MH805845 & MH781475 & MH846558 & de Silva et al. (2019) \\
\hline C. theobromicola & ICMP 18649 & JX010294 & JX010006 & JX009869 & JX009444 & JX010447 & Rojas et al. (2010) \\
\hline C. temperatum & CBS 133122 & JX145159 & & & & JX145211 & Doyle et al. (2013) \\
\hline C. $t i$ & ICMP 4832 & JX010269 & JX009952 & JX009898 & JX009520 & JX010442 & Weir et al. (2012) \\
\hline C. tropicale & CBS 124949 & JX010264 & JX010007 & JX009870 & JX009489 & JX010407 & Rojas et al. (2010) \\
\hline C. viniferum & GZAAS5.08601 & JN412804 & JN412798 & & JN412795 & JN412813 & Peng et al. (2013) \\
\hline C. wuxiense & CGMCC 3.17894 & KU251591 & KU252045 & KU251939 & KU251672 & KU252200 & Wang et al. (2016) \\
\hline C. xanthorrhoeae & ICMP 17903 & JX010261 & JX009927 & JX009823 & JX009478 & JX010448 & Shivas et al. (1998) \\
\hline C. yulongense & CFCC 50818 & MH751507 & MK108986 & MH793605 & MH777394 & MK108987 & Wang et al. (2019) \\
\hline
\end{tabular}

Colletotrichum aeschynomenes B. Weir \& P.R. Johnst., Stud. Mycol. 73: 135 (2012)

Sexual morph not reported. See Weir et al. (2012) for illustrations and descriptions of asexual morph.

Type - USA, Arkansas, on Aeschynomene virginica (stem lesion), D. TeBeest 3-1-3, holotype PDD 101995, culture ex-type ICMP 17673.

Hosts - Aeschynomene virginica (Weir et al. 2012), Theobroma cacao (Nascimento et al. 2019), Manihot esculenta (Sangpueak et al. 2018), Myrciaria dubia (Matos et al. 2020)

Distribution - USA (Weir et al. 2012), Thailand (Sangpueak et al. 2018), Brazil (Nascimento et al. 2019, Matos et al. 2020)

Notes - This taxon can be distinguished by $t u b 2$, gapdh, or $g s$ sequence data. Genetically close to $C$. siamense and differs in having slightly longer and narrower conidia which taper slightly toward the ends and in having larger, strongly lobed appressoria (Weir et al. 2020). Pathogenicity studies are available for this species.

Colletotrichum alatae B. Weir \& P.R. Johnst., Stud. Mycol. 73: 135 (2012)

Sexual morph not reported. See Weir et al. (2012) for illustrations and descriptions of asexual morph.

Type - India, Rajasthan, Udaipur, on Dioscorea alata leaves and stems, K.L. Kothari \& J. Abramham, 1959, holotype CBS H-6939, culture extype CBS 304.67. 
Hosts - Dioscorea alata (Weir et al. 2012)

Distribution - Barbardos, Guadeloupe, India, Nigeria (Weir et al. 2012)

Notes - This species can be distinguished from other species based on ITS sequence data (Weir et al. 2012). Even though this species was recorded as a pathogen on yam, no data are available to confirm its pathogenicity.

Colletotrichum alienum B. Weir \& P.R. Johnst., Stud. Mycol. 73: 139 (2012)

See Weir et al. (2012) for illustrations and descriptions of asexual and sexual morphs.

Type - New Zealand, Auckland, Kumeu research orchard, Malus domestica (fruit rot), P.R. Johnston C824, 14 Aug. 1987, holotype PDD 101996, culture ex-type ICMP 12071.

Hosts - wide range of hosts (including Banksia dryandroides, Camellia sinensis, Diospyros kaki, Grevillea sp., Leucospermum sp., Malus domestica, Nerium oleander, Persea americana, Protea sp., Serruria sp. Telopea sp. (Weir et al. 2012), Aquilaria sinensis (Liu et al. 2020a)

Distribution - Australia, China, Hawaii, New Zealand, Portugal, South Africa and Zimbabwe (Weir et al. 2012, Crous et al. 2013, Liu et al. 2013b, 2015a, Schena et al. 2014, Liu et al. 2020a)

Notes - This taxon is best distinguished using cal or $g s$ sequence data (Weir et al. 2012). It is common on commercial fruits and its pathogenicity has been confirmed.

Colletotrichum aotearoa B. Weir \& P.R. Johnst., Stud. Mycol. 73: 139 (2012)

Sexual morph not reported. See Weir et al. (2012) for illustrations and descriptions of asexual morph.

Type - New Zealand, Auckland, Glen Innes, Auckland University campus, on Coprosma sp. (incubated berries), B. Weir C1282.4,30 Apr 2009, holotype PDD 101076, culture ex-type ICMP 18537.

Hosts - Banksia marginata, Boehmeria sp., Bredia oldhamii, Coprosma sp., Dacrycarpus dacrydioides, Knightia sp., Musa sp., Podocarpus totara, Vitex lucens (Weir et al. 2012)

Distribution - New Zealand (Weir et al. 2012)

Notes - It is common on taxonomically diverse native plants in New Zealand as a pathogen causing fruit rot and also as an endophyte on naturalized weeds. This species seems to be restricted only to New Zealand. Sequence data of $t u b 2$, cal, gs, and gapdh sequence data can be used to differentiate this taxon from other species.

Colletotrichum arecicola X.R. Cao, H.Y. Che \& D.Q. Luo, in Cao et al., Pl. Dis. 104(5): 1371 (2020)

Sexual morph not reported. See Cao et al. (2020) for illustrations and descriptions of asexual morph.

Type - China, Hainan Province, Wenchang city, on a diseased areca palm leaf (Areca catechu), March. 2019, X.R. Cao, holotype HMAS 248158, culture ex-type CGMCC 3.19667.

Hosts - Areca catechu (Cao et al. 2020)

Distribution - China (Cao et al. 2020)

Notes - Colletotrichum arecicola is closely related with $C$. cordylinicola, but can be separated from having longer appressoria and knobbed appressoria on PDA (see Cao et al. 2020). Pathogenicity data for this species is available.

Colletotrichum artocarpicola Bhunjun, Jayawardena, Jeewon \& K.D. Hyde, in Bhunjun et al., Phytotaxa 418(3): 279 (2019)

Sexual morph not reported. See Bhunjun et al. (2019) for illustrations and descriptions of asexual morph.

Type - Thailand, Chiang Rai, on decaying root of Artocarpus heterophyllus, R.S. Jayawardena, 2018, holotype MFLU 18-1167, culture ex-type MFLUCC 18-1167.

Hosts - Artocarpus heterophyllus (Bhunjun et al. 2019)

Distribution - Thailand (Bhunjun et al. 2019) 
Notes - Colletotrichum artocarpicola is similar to C. endophytica, and can be distinguished by septate germinating conidia (Bhunjun et al. 2019). Pathogenicity studies for this species are available. This taxon can be distinguished by its gapdh and act sequence data.

Colletotrichum asianum Prihastuti, L. Cai \& K.D. Hyde, Fungal Diversity 39: 96 (2009)

See Prihastuti et al. (2009) for illustrations and descriptions of asexual and sexual morph.

Type - Thailand, Chiang Mai Province, Mae Taeng District, Pha Daeng Village, near Mushroom Research Centre, on berry of Coffea arabica, 12 December 2007, H. Prihastuti, holotype MFLU 09-0228, culture ex-type ICMP 18580.

Hosts - Coffea arabica, Mangifera indica (Weir et al. 2012)

Distribution - Australia, Colombia, Japan, Panama, Philippines, Thailand (Weir et al. 2012)

Notes - This taxon is recorded as a pathogen on mango and coffee. Its pathogenicity has been confirmed through pathogenicity studies. Colletotrichum asianum can be distinguished by any of the loci (ITS, gapdh, cal, act, chs-1, gs, sod2 and tub2) used in Weir et al. (2012).

Colletotrichum camelliae Massee, Bull. Misc. Inf., Kew: 91 (1899)

Sexual morph not reported. See Liu et al. (2015a) for illustrations and descriptions of asexual morph.

Type - China, Guizhou Province, Huishui District, on Camellia sinensis, 11 Nov. 2010, P. Tan, epitype HMAS 243126, culture ex-epitype CGMCC 3.14925.

Hosts - Camellia sinensis (Liu et al. 2015a)

Distribution - China (Liu et al. 2015a)

Notes - Colletotrichum camelliae belongs to the kahawae clade within the gloeosporioides complex and can be distinguished with the use of $g s$ and apmat sequence data (Liu et al. 2015). This is recorded as the causal agent of the brown blight disease of tea plants (Weir et al. 2012). The type of this species was recorded from Sri Lanka. Even though there are 36 records in Farr \& Rossman (2021), only the strains used in Liu et al. (2015a) have molecular data. More collections are needed to confirm the records that were identified based on morphology alone.

Colletotrichum changpingense Guo Z. Zhang, Jayaward. \& K.D. Hyde, in Jayawardena et al., Mycosphere 7(8): 1155 (2016)

Sexual morph not reported. See Jayawardena et al. (2016b) for illustrations and descriptions of asexual morph.

Type - China, Beijing City, Changping, Xingshou Town, from rhizome of Fragaria $\times$ ananassa, November 2011, Zhang Guozhen, holotype MFLU 15-0212, culture ex-type MFLUCC 15-0022.

Hosts - Fragaria $\times$ ananassa (Jayawardena et al. 2016b)

Distribution - China (Jayawardena et al. 2016b)

Notes - Colletotrichum changpingense is phylogenetically closely related to C. grevilleae, $C$. grossum and $C$. theobromicola and can be differentiate by any of the loci used on Jayawardena et al. (2016b). Its pathogenicity on strawberry fruits, leaves and rhizome has been confirmed through pathogenicity studies.

Colletotrichum chrysophilum W.A.S. Vieira, W.G. Lima, M.P.S. Câmara \& V.P. Doyle, Mycologia 109(6): 927 (2017)

Sexual morph not reported. See Vieira et al. (2017) for illustrations and descriptions of asexual morph.

Type - Brazil, SÃO PAULO, Registro, a dried culture of a strain isolated from Musa sp. cv. 'Ouro' (fruit anthracnose), July 2013, W.G. Lima, holotype URM89949, culture ex-type CMM4268.

Hosts - Musa sp. (Vieira et al. 2017)

Distribution - Brazil (Vieira et al. 2017) 
Notes - Colletotrichum chrysophilum can be distinguished from C. fructicola and C. nupharicola by act, apn2, apn2/mat-igs, cal, gap2-igs, gapdh, gs and tub2 sequence data (Vieira et al. 2017).

Colletotrichum cigarro (B.S. Weir \& P.R. Johnst.) A. Cabral \& P. Talhinhas, in Cabral et al., Plants, (Basel) 9(4, no. 502): 12 (2020)

See Cabral et al. (2020) for illustrations and descriptions of asexual and sexual morphs.

Type - Australia, on Olea europaea, V. Sergeeva UWS124, 1989, holotype PDD 102232, culture ex-type ICMP 18539.

Hosts - Olea europaea (Cabral et al. 2020), Persea Americana (Fuentes-Aragon et al. 2020)

Distribution - Australia (Cabral et al. 2020), Mexico (Fuentes-Aragon et al. 2020)

Notes - Weir et al. (2012) introduced this taxon as C. kahawae supsp. ciggaro. Cabral et al. (2020) upgraded it as a species. This species is recorded as a pathogen and the pathogenicity has been confirmed by pathogenicity tests.

Colletotrichum clidemiae B.S. Weir \& P.R. Johnst., in Weir et al., Stud. Mycol. 73: 148 (2012)

Sexual morph not reported. See Weir et al. (2012) for illustrations and descriptions of asexual morph.

Type - USA, Hawai'i, Aiea, on Clidemia hirta (leaf spot), S.A. Ferreira \& K. Pitz, 14 May 2010, holotype PDD 101997, culture ex-type ICMP 18658.

Hosts - Clidemia hirta, Vitis sp., Quercus sp. (Weir et al. 2012)

Distribution - Panama, USA (Weir et al. 2012)

Notes - This species causes leaf spots and belongs to the kahawae clade within the gloeosporioides species complex (Weir et al. 2012). Colletotrichum clidemiae can be distinguished by act, gapdh or gs sequence data (Weir et al. 2012) as well as apmat (Jayawardena et al. 2016a).

Colletotrichum conoides Y.Z. Diao, C. Zhang, L. Cai \& Xi L. Liu, in Diao et al., Persoonia 38: 27 (2017)

See Fu et al. (2019) for illustrations and descriptions of sexual morph. See Damm et al. (2019) for illustrations and descriptions of asexual morph.

Type - China, Jiangsu Province, Nanjing City, on fruits of Capsicum annuum var. conoides, Sept. 2010, Y.Z. Diao, holotype HMAS 246481, ex-type culture CGMCC 3.176156.

Hosts - Capsicum annuum (Diao et al. 2017), Pyrus pyrifolia (Fu et al. 2019)

Distribution - China (Diao et al. 2017, Fu et al. 2019)

Notes - Colletotrichum conoides is phylogenetically most closely related to C. hebeiense. Sequence data from gapdh, act, or tub2 can be used to distinguish this species (Diao et al. 2017).

Colletotrichum cobbittiense S. Luo, G. Dong \& P. Wong, in Crous et al., Persoonia 40: 271 (2018)

Sexual morph not reported. See Crous et al. (2018b) for illustrations and descriptions of asexual morph.

Type - Australia, New South Wales, Cobbitty, from leaf lesions of a Cordyline interspecific hybrid (C. stricta $\times$ C. australis), Jan. 2016, S. Luo \& G. Dong, holotype BRIP 66219.

Hosts - Cordyline sp. (Crous et al. 2018b)

Distribution - Australia (Crous et al. 2018b)

Notes - The leaf lesions were characterised by bleached centres and diffuse brownish margins around the lesions (Crous et al. 2018b). Pathogenicity studies are needed to confirm its pathogenicity. This taxon can be distinguished by any of the loci (ITS, gapdh, chs-1, act and tub2) used in Crous et al. (2018b).

Colletotrichum cordylinicola Phoulivong, L. Cai \& K. D. Hyde, Mycotaxon 114: 251 (2011)

Sexual morph not reported. See Phoulivong et al. (2010) for illustrations and descriptions of asexual morph. 
Type - Panama, Gamboa, wet lowland forest, leaf endophyte of Merremia umbellata, Nov. 2004, S. Van Bael \& Z. Maynard, D2-13, holotype CBS H-21066, culture ex-type CBS 125386.

Hosts - Eugenia javanica (Phoulivong et al. 2010), Cordyline fruticose (Weir et al. 2012), Areca catechu (Cao et al. 2020)

Distribution - Laos (Phoulivong et al. 2010), Thailand, USA (Weir et al. 2012), China (Cao et al. 2020)

Notes - This taxon can be identified by ITS sequence data (Weir et al. 2012). Pathogenicity data are available for this species.

Colletotrichum endophytica Manamgoda, Udayanga, L. Cai \& K.D. Hyde, in Manamgoda et al., Fungal Diversity 61:110 (2013)

Sexual morph not reported. See Manamgoda et al. (2013) for illustrations and descriptions of asexual morph.

Type - Thailand, Chiang Rai Province, endophytic on healthy leaves of Pennisetum purpureum, 5 May 2010, Dimuthu S. Manamgoda, holotype MFLU13-0004 (dried culture), culture ex-type MFLUCC 130418.

Hosts - Pennisetum purpureum (Manamgoda et al. 2013)

Distribution - Thailand (Manamgoda et al. 2013)

Notes - This is an endophytic species on healthy leaves of Pennisetum purpureum in Thailand (Manamgoda et al. 2013). It was also found as a saprobe on an undetermined wild fruit in Thailand (Udayanga et al. 2013). Colletotrichum endophytica belongs to the gloeosporioides species complex and placed in between the musae and kahawae clades (Manamgoda et. al. 2013).

Colletotrichum fructicola Prihastuti, L. Cai \& K.D. Hyde, Fungal Diversity 39: 96 (2009)

See Fu et al. (2019) for illustrations and descriptions of sexual morph. See Rojas et al. (2010) for illustrations and descriptions of asexual morph.

Type - Panama, Gamboa, wet lowland forest, leaf endophyte of Merremia umbellata, Nov. 2004, S. Van Bael \& Z. Maynard, D2-13, holotype CBS H-21066, culture ex-type CBS 125386.

Hosts - wide range (Farr \& Rossman 2021)

Distribution - worldwide (Farr \& Rossman 2021)

Notes - Colletotrichum fructicola was originally reported from coffee berries in Thailand (Prihastuti et al. 2009). It has a wide host range and geographical distribution (Jayawardena et al. 2016a). These taxa are best distinguished using $g s$ or $\operatorname{sod} 2$ sequence data (Weir et al. 2012). It is recorded as a pathogen, endophyte and a saprobe. Pathogenicity data are available for this species.

Colletotrichum fructivorum V.P. Doyle, P.V. Oudem. \& S.A. Rehner, PLoS ONE 7(12): e51392, 12 (2012)

Sexual morph not reported. See Doyle et al. (2013) for illustrations and descriptions of asexual morph.

Type - USA, New Jersey, Burlington County, Vaccinium macrocarpon (fruit-rot pathogen), Oct 2010, V. Doyle, P.V. Oudemans, C. Constantelos, holotype BPI 884103, culture ex-type CBS 133125.

Hosts - Vaccinium macrocarpon, Rhexia virginica (Doyle et al. 2013)

Distribution - Canada, Colombia and the USA (Doyle et al. 2013)

Notes - This taxon is pathogenic to Vaccinium sp. in Canada, Colombia and the USA (Doyle et al. 2013). Doyle et al. (2013) reported this species as an endophyte on Rhexia virginica and Vaccinium macrocarpon in the USA.

Colletotrichum gloeosporioides (Penz.) Penz. \& Sacc., Atti Inst. Veneto Sci. lett., ed Arti, Sér. 6 (2): 670 (1884)

Sexual morph not reported. See Cannon et al. (2008) for illustrations and descriptions of asexual morph. 
Type - Italy, Calabria, on Citrus sinensis, culture ex-epitype IMI 356878.

Hosts - Citrus sp., Carya illinoinensis, Ficus sp., Mangifera sp. Solanum betaceum, Pureraria sp., Vitis vinifera (Weir et al. 2012) 2012)

Distribution - Australia, China, Colombia, New Zealand, South Africa, USA (Weir et al.

Notes - Weir et al. (2012) did a comprehensive study on C. gloeosporioides and related species, resolving most of the species under the name of $C$. gloeosporioides. Mostly it is associated with Citrus sp. (Weir et al. 2012). This species can be separated from all other species by ITS sequence data.

Colletotrichum grevilleae F. Liu, Damm, L. Cai \& Crous, Fungal Diversity 61: 98 (2013) morph.

Sexual morph not reported. See Liu et al. (2013b) for illustrations and descriptions of asexual

Type - Italy, Catania, Grevillea sp. (from root and collar rot), Jan. 2000, G. Polizzi, holotype CBS H-21120, culture ex-type CBS 132879.

Hosts - Grevillea sp. (Liu et al. 2013b)

Distribution - Italy (Liu et al. 2013b)

Notes - This species is a pathogen. However, pathogenicity studies are not available to confirm its pathogenicity (Liu et al. 2013b). It has a close affinity with $C$. theobromicola. However, these species can be distinguished based on cal and gapdh sequence data (Liu et al. 2013b).

Colletotrichum grossum Y.Z. Diao, C. Zhang, L. Cai \& X.L. Liu, Persoonia 38: 29 (2017) morph.

Sexual morph not reported. See Diao et al. (2017) for illustrations and descriptions of asexual

Type - China, Hainan Province, Haikou city, on Capsicum annuum var. grossum, Oct. 2010, Y.Z Diao, holotype HMAS 246480, culture ex-type CGMCC3.17614.

Hosts - Capsicum annuum (Diao et al. 2017), Mangifera indica (Leon et al. 2018)

Distribution - China (Diao et al. 2017), Cuba (Leon et al. 2018)

Notes - Colletotrichum grossum is phylogenetically most closely related to C. theobromicola, and can be distinguished based on gapdh, act and tub2 sequence data. Pathogenicity studies are available for this species.

Colletotrichum hebeiense X.H. Li, Y. Wang, K.D. Hyde, M.M.R.S. Jayawardena \& J.Y. Yan, in Yan et al., Fungal Diversity 71: 241 (2015)

Sexual morph not reported. See Yan et al. (2015) for illustrations and descriptions of asexual morph.

Type - China, Hebei province, Qinhuangdao City, on fruits of Vitis vinifera cv. 'Cabernet Sauvignon', September 2009, X.H. Li, holotype MFU14-0627, culture ex-type MFLUCC 13-0726.

Hosts - Vitis vinifera (Yan et al. 2015)

Distribution - China (Yan et al. 2015)

Notes - This is a pathogen on Vitis vinifera in China. Pathogenicity data are available for this species (Yan et al. 2015).

Colletotrichum hederiicola Jayaward. Camporesi \& K.D. Hyde, in Hyde et al., Fungal Diversity 100: 193 (2020)

Sexual morph not reported. See Hyde et al. (2020) for illustrations and descriptions of asexual morph.

Type - ITALY, Province of Forlì-Cesena [FC], Fiumana di Predappio, on dead branch of Hedera helix (Araliaceae), 28 October 2014, E. Camporesi, IT 2201 (MFLU 15-0689, holotype).

Hosts - Hedera helix (Hyde et al. 2020)

Distribution - Italy (Hyde et al. 2020) 
Notes - This species has a close affinity with $C$. henanense and can be separated from chs- 1 and act sequence data. Culture is not available for this species.

Colletotrichum helleniense Guarnaccia \& Crous, in Guarnaccia et al., Persoonia 39: 43 (2017)

Sexual morph not reported. See Guarnaccia et al. (2017) for illustrations and descriptions of asexual morph.

Type - Greece, Arta, from wither-tip twigs of Poncirus trifoliata, 20 May 2015, V. Guarnaccia (CBS H-23025 holotype, culture ex-type CBS 142418.

Hosts - Citrus reticulata, Poncirus trifoliate (Guarnaccia et al. 2017)

Distribution - Greece (Guarnaccia et al. 2017)

Notes - Colletotrichum helleniense was isolated from fruit lesions of Citrus reticulata and from wither-tip twigs Poncirus trifoliata in Greece. Both these genera are in Rutaceae, which shows the ability of $C$. helleniense to colonise tissues of different genera within the Rutaceae. This species is phylogenetically closer to C. kahawae but can be differentiated based on gapdh, cal and tub2 sequence data (Guarnaccia et al. 2017).

Colletotrichum henanense F. Liu \& L. Cai, in Liu et al., Persoonia, Mol. Phyl. Evol. Fungi 35: 80 (2015)

Sexual morph not reported. See Liu et al. (2015) for illustrations and descriptions of asexual morph.

Type - China, Henan Province, Xinyang, on Camellia sinensis, 23 Sept. 2012, M. Zhang \& R. Zang, holotype HMAS 245381, culture ex-type CGMCC 3.17354. 2018)

Hosts - Camellia sinensis, Cirsium japonicum (Liu et al. 2015a), Camellia oleifera (Li et al.

Distribution - China (Liu et al. 2015a, Li et al. 2018)

Notes - This species can be distinguished can be distinguished by tub2, apmat and $g s$ sequence data (Liu et al. 2015a). Pathogenicity studies have been carried out to establish its pathogenicity.

Colletotrichum horii B. Weir \& P.R. Johnst., Mycotaxon 111: 211 (2010)

Sexual morph not reported. See Weir \& Johnston (2010) for illustrations and descriptions of asexual morph.

Type - Japan, on Diospyros kaki, N. Nishihara A71, 1959, neotype TNS-F-26102 (dried culture), culture neotype ICMP 10492.

Hosts - Diospyros kaki (Weir \& Johnston 2010)

Distribution - Brazil, China, Japan, Korea, New Zealand, South Korea (Farr \& Rossman 2021)

Notes - This species is recorded as associated with unripe fruits, young stem and twig lesions (Weir \& Johnston 2010). Xie et al. (2010) noted minor symptoms on inoculated fruit of Capsicum annuum, Musa acuminata and Cucurbita pepo. However, this taxon had never been associated with disease symptoms on these hosts from the field. It can be identified based on ITS sequence data.

Colletotrichum hystricis Guarnaccia \& Crous, in Guarnaccia, Groenewald, Polizzi \& Crous, Persoonia 39: 43 (2017)

Sexual morph not reported. See Guarnaccia et al. (2017) for illustrations and descriptions of asexual morph.

Type - Italy, Mascali, Catania, from leaf lesion of Citrus hystrix, 30 Jan. 2016, V. Guarnaccia holotype CBS H-23026, culture ex-type CBS 142411.

Hosts - Citrus hystrix (Guarnaccia et al. 2017)

Distribution - Italy (Guarnaccia et al. 2017)

Notes - Colletotrichum hystricis was isolated from a leaf lesion, however there are no 
pathogenicity data available for this. This species differs from closely related species in gapdh, act and chs-1 sequence data (Guarnaccia et al. 2017).

Colletotrichum jiangxiense F. Liu \& L. Cai, in Liu et al., Persoonia 35: 82 (2015)

Sexual morph not reported. See Liu et al. (2015a) for illustrations and descriptions of asexual morph.

Type - China, Jiangxi Province, Ganzhou, Fengshan Mountain, on Camellia sinensis, Sept. 2013, Y. Zhang, holotype HMAS 245382, culture ex-type CGMCC 3.17363.

Hosts - Camellia sinensis (Liu et al. 2015a)

Distribution - China (Liu et al. 2015a)

Notes - This species is a pathogen as well as an endophyte on Camellia sinensis in China. It is closely related to C. kahawae sensu lato. Colletotrichum jiangxiense can be distinguished by gs gene sequence data (Liu et al. 2015a).

Colletotrichum kahawae J.M Waller \& Bridge, Mycol. Res. 97(8): 993 (1993)

Sexual morph not reported. See Waller et al. (1993) for illustrations and descriptions of asexual morph.

Type -Kenya, on berries of Coffea arabica, 1987-01-29, holotype IMI 319418, culture extype ICMP17816.

Hosts - Coffea arabica (Waller et al. 1993, Weir et al. 2012)

Distribution - African continent (Waller et al. 1993, Weir et al. 2012)

Notes - This species was introduced to accommodate the species causing coffee berry disease (Waller et al. 1993). Colletotrichum kahawae apparently is restricted to and widespread on coffee in Africa (Weir et al. 2012). This taxon has a distinctive growth form and biology (Waller et al. 1993). Colletotrichum kahawae can be distinguished by its ITS and $g s$ sequence data.

Colletotrichum makassarense D.D. de Silva, Crous \& P.W.J. Taylor, in de Silva et al., IMA Fungus 10(1): 23 (2019)

Sexual morph not reported. See de Silva et al. (2019) for illustrations and descriptions of asexual morph.

Type - Indonesia, Makassar, from fruit lesion of Capsicum annuum, 7 Jun. 2015, P.W.J. Taylor \& A. Nasruddin, holotype CBS H-143664, culture ex-type CBS 143664.

Hosts - Capsicum annuum (de Silva et al. 2019)

Distribution - Indonesia (de Silva et al. 2019)

Notes - This species is only recorded from Capsicum annuum and can be distinguished by apmat and $g s$ sequence data (de Silva et al. 2019).

Colletotrichum musae (Berk. \& M. A. Curtis) Arx, Verh. K. ned. Akad. Wet., tweede sect. 51(3): 107 (1957)

Sexual morph not reported. See Su et al. (2011) for illustrations and descriptions of asexual morph.

Type - USA, Florida, on Musa sp., isolated by M. Arzanlou, holotype CBS-H-20515 (driedculture), culture ex-epitype CBS 116870.

Hosts - Musa sp. (Su et al. 2011, Weir et al. 2012)

Distribution - worldwide (Su et al. 2011, Weir et al. 2012, Jayawardena et al. 2016a)

Notes - Colletotrichum musae is recorded as a pathogen and an endophyte of Musa sp. worldwide ( $\mathrm{Su}$ et al. 2011, Weir et al. 2012, Jayawardena et al. 2016a). This taxon can be distinguished from ITS sequence data (Weir et al 2012).

Colletotrichum nupharicola D.A. Johnson, Carris \& J.D. Rogers, Mycol. Res. 101(6): 647 (1997)

Sexual morph not reported. See Johnson et al. (1997) for illustrations and descriptions of asexual morph. 
Type - USA, Washington, Yakima Co., on Nupha lutea subsp. polysepala, D.A. Johnson A2, Oct. 1993, holotype WSP 69656, culture ex-holotype CBS 470.96.

Hosts - Nuphar, Nymphae (Johnston et al. 1997)

Distribution - USA (Johnston et al. 1997)

Notes - This species is a pathogen recorded only in the USA, on the aquatic plants Nuphar and Nymphae sp. (Johnston et al. 1997). This species can be distinguished by all loci (gapdh, cal, chs-1, act, gs, sod2 and tub2) used in Weir et al. (2012), except ITS.

Colletotrichum pandanicola Tibpromma \& K.D. Hyde, in Tibpromma et al., MycoKeys 33: 47 (2018)

Sexual morph not reported. See Tibpromma et al. (2018) for illustrations and descriptions of asexual morph.

Type - Thailand, Chumphon, Pathio District, on healthy leaves of Pandanus sp., 1 December 2016, S. Tibpromma (PE09), holotype MFLU 18-0003, culture ex-type, MFLUCC 17-0571.

Hosts - Pandanus sp. (Tibpromma et al. 2018)

Distribution - Thailand (Tibpromma et al. 2018)

Notes - It is recorded as an endophyte and can be identified by all the loci (ITS, gapdh, act, chs-1 and tub2) used in Tibpromma et al. (2018) except for ITS.

Colletotrichum persea G. Sharma \& S. Freeman, in Sharma et al., Scientific Reports 7(no. 15839): 7 (2017)

See Sharma et al. (2017) for illustrations and descriptions of asexual and sexual morphs.

Type - Israel, Mikve Israel, (central Israel), on Persea Americana cv. 'Hass' (post-harvest ripe fruit rot), S. Freeman (GA100 1-12-2014), holotype HUJIHERB-902850-FUNGI, culture exholotype CBS 141365.

Hosts - Persea Americana (Sharma et al. 2017)

Distribution - Israel (Sharma et al. 2017)

Notes - Colletotrichum perseae can be well-resolved using apmat and gs markers (Sharma et al. 2017). Pathogenicity studies are available for this species.

Colletotrichum pseudotheobromicola Chethana, J.Y. Yan, X.H. Li \& K.D. Hyde, in Chethana et al., Mycosphere 10(1): 518 (2019)

Sexual morph not reported. See Chethana et al. (2019) for illustrations and descriptions of asexual morph.

Type - China, Beijing, on leaf spots of Prunus avium, 28 September 2017, K.W.T. Chethana holotype MFLU 18-2656, culture ex-type, MFLUCC 18-1602.

Hosts - Prunus avium (Chethana et al. 2019)

Distribution - China (Chethana et al. 2019)

Notes - This taxon can be distinguished by act and gapdh sequence data (Chethana et al. 2019). Pathogenicity data are available for this species.

Colletotrichum protea F. Liu, Damm, L. Cai \& Crous, Fungal Diversity 61: 100 (2013) morph.

Sexual morph not reported. See Liu et al. (2013b) for illustrations and descriptions of asexual

Type - South Africa, Western Cape Province, Tsitsikamma National Park, Nature's Valley, on Protea sp., 9 Jan. 2008, P.W. Crous, holotype CBS H-21119, culture ex-type CBS 132882.

Hosts - Protea sp. (Liu et al. 2013b)

Distribution - South Africa (Liu et al. 2013b)

Notes - Although the conidial morphology of this species is similar to the acutatum species complex, phylogenetically it places in the gloeosporioides complex. It is recorded only on Protea sp. 
Colletotrichum psidii Curzi, Atti Ist. bot. R. Univ. Pavia, 3 Sér. 3(3): 207 (1927) morph.

Sexual morph not reported. See Weir et al. (2012) for illustrations and descriptions of asexual

Reference specimen - Italy, Rome, on Psidium sp., M. Curzi, authentic culture CBS 145.29

Hosts - Psidium sp. (Weir et al. 2012)

Distribution - Italy (Weir et al. 2012)

Notes - This is only recorded from its authentic strain and can be distinguished by ITS sequence data (Weir et al. 2012).

Colletotrichum queenslandicum B. Weir \& P.R. Johnst., in Weir et al., Stud. Mycol. 73: 164 (2012) morph.

Sexual morph not reported. See Weir et al. (2012) for illustrations and descriptions of asexual

Type - Australia, Queensland, Brisbane, on Carica papaya, J.H. Simmonds 11663C, Sep. 1965, epitype PDD 28797, culture ex-epitype ICMP 1778.

Hosts - Anacardium occidentale, Capsicum annuum, Carica papaya, Citrus latifolia, Coffea sp., Licania tomentosa, Litchi chinensis, Mangifera indica, Nephelium lappaceum, Olea europaea, Passiflora edulis, Persea amaericana (Weir et al. 2012, Farr \& Rossman 2021)

Distribution - Australia, Brazil, Fiji, Puerto Rico, USA (Weir et al. 2012, Farr \& Rossman 2021)

Notes - This is a pathogen and pathogenicity studies are available. Colletotrichum queenslandicum belongs to the musae clade. It can be best distinguished with the use of tub2, gapdh and gs sequence data (Weir et al. 2012).

Colletotrichum rhexiae Ellis \& Everh., Proc. Acad. nat. Sci. Philad. 46: 372 (1894)

Sexual morph not reported. See Doyle et al. (2013) for illustrations and descriptions of asexual morph.

Type - USA, Sussex County, Delaware, Cape Henlopen State Park, Rhexia virginica (stem tissue lesion), Nov 2010, V. Doyle, epitype Coll1026, culture ex-epitye CBS 133134).

Hosts - Rhexia virginica, Vaccinium macrocarpon (Doyle et al. 2013)

Distribution - USA (Doyle et al. 2013)

Notes - This species is recorded as a pathogen of Rhexia sp. as well as a fruit endophyte on Vaccinium macrocarpon in the USA (Doyle et al. 2013). It is in the kahawae clade and can be identified any of the loci (ITS and tub2) used in Doyle et al. (2013).

Colletotrichum salsolae Weir \& P.R. Johnst., in Weir et al., Stud. Mycol. 73: 164 (2012)

Sexual morph not reported. See Weir et al. (2012) for illustrations and descriptions of asexual morph.

Type - Hungary, on Salsola tragus, coll. D. Berner, 1996, holotype BPI 878740, culture exholotype ICMP 19051.

Hosts - Salsola tragus (Weir et al. 2012)

Distribution - Hungary (Weir et al. 2012)

Notes - This species can be distinguished by closely related species from gapdh and tub2 sequence data. There is a record of $C$. salsolae causing disease on Carica papaya in India. However, the analyses were carried out based on ITS, which cannot be used to identify this species accurately.

Colletotrichum siamense Phoulivong, L. Cai \& K.D. Hyde, in Prihastuti et al., Fungal Diversity 39: 98 (2009)

See Fu et al. (2019) for illustrations and descriptions of sexual morph. See Prihastuti et al. (2009) for illustrations and descriptions of asexual morph. 
Type - Thailand, Chiang Mai Province, Mae Taeng District, Mae Lod Village, Royal Agricultural Project Coffee, on berry of Coffea arabica, 16 January 2008, H. Prihastuti holotype MFLU 090230, culture ex-type ICMP 18578.

Hosts - wide host range (see Farr \& Rossman 2021)

Distribution - worldwide (see Farr \& Rossman 2021)

Notes - Colletotrichum siamense was introduced from Coffee berries in Thailand. With more collections, it is clear that this has a wide host range a geographical distribution. Pathogenicity studies are available for this species. It is better distinguished by $c a l$ and $t u b 2$ sequence data (Weir et al. 2012).

Colletotrichum syzygicola Udayanga, Manamgoda \& K.D. Hyde [as 'syzygicola'], in Udayanga et al., Fungal Diversity 61: 173 (2013)

Sexual morph not reported. See Udayanga et al. (2013) for illustrations and descriptions of asexual morph.

Type - Thailand, Chiang Rai Province, Nang Lae, Fah-Thai market, on fruits of Syzygium samarangense, 18 April 2010, Dhanushka Udayanga, holotype MFLU12-2476 (dried sporulating culture on PDA), culture ex type MFLUCC 10-0624.

Hosts - Citrus aurantifolia, Syzygium samarangense (Udayanga et al. 2013)

Distribution - Thailand (Udayanga et al. 2013)

Notes - This species is recorded as a pathogen, however no pathogenicity studies are available to confirm it. Colletotrichum syzygicola can be differentiated by any of the loci (ITS, gapdh, act and tub2) used in Udayanga et al. (2013).

Colletotrichum tainanense D.D. de Silva, Crous \& P.W.J. Taylor, in de Silva et al., IMA Fungus 10(1): 23 (2019)

Sexual morph not reported. See de Silva et al. (2019) for illustrations and descriptions of asexual morph.

Type - Taiwan, Tainan: on fruit of Capsicum annuum, Aug. 2014, Z.M. Sheu, holotype CBS H-143666, culture ex-type CBS 143666.

Hosts - Capsicum annuum (de Silva et al. 2019)

Distribution - Taiwan (de Silva et al. 2019)

Notes - This taxon is recorded only from the type strain and can be distinguished by tub2 sequence data (de Silva et al. 2019).

Colletotrichum theobromicola Delacr., Bull. Soc. mycol. Fr. 21: 191 (1905)

Sexual morph not reported. See Rojas et al. (2010) for illustrations and descriptions of asexual morph.

Type - Panama, Gamboa, wet lowland forest, leaf endophyte of Merremia umbellata, Nov. 2004, S. Van Bael \& Z. Maynard, D2-13, holotype CBS H-21066, culture ex-type CBS 125386.

Hosts - wide range of hosts (see Farr \& Rossman 2021)

Distribution - worldwide (see Farr \& Rossman 2021)

Notes - Colletotrichum theobromicola was accepted by Weir et al. (2012) as putatively specialized pathogens. Pathogenicity studies are available for this species and can be differentiated by ITS sequence data (Weir et al. 2012).

Colletotrichum temperatum V. Doyle, P.V. Oudem. \& S.A. Rehner, PLoS ONE 7(12): e51392, 17 (2012)

Sexual morph not reported. See Doyle et al. (2013) for illustrations and descriptions of asexual morph.

Type - USA, New York, Bronx County, The New York Botanical Garden, Vaccinium macrocarpon (rotten fruit), Nov 2009, V. Doyle \& C. Mozzicato, culture ex-type CBS 133122.

Hosts - Vaccinium macrocarpon (Doyle et al. 2013) 
Distribution - USA (Doyle et al. 2013)

Notes - Colletotrichum temperatum is an endophyte and a pathogen on Vaccinium macrocarpon. This species can be distinguished by any of the loci (ITS and tub2) used in Doyle et al. (2013).

Colletotrichum ti B. Weir \& P.R. Johnst., in Weir et al., Stud. Mycol. 73: 171 (2012)

See Weir et al. (2012) for illustrations and descriptions of asexual and sexual morphs.

Type - New Zealand, Taupo, on Cordyline sp., coll. J.M. Dingley 65187, Sep. 1965, holotype PDD 24881, culture ex-holotype ICMP 4832.

Hosts - Cordyline sp. (Weir et al. 2012)

Distribution - New Zealand (Weir et al. 2012)

Notes - This is recorded only from the type strain. Although this was isolated from a leaf lesion, no pathogenicity data to confirm whether this species is a pathogen. Colletotrichum ti can be distinguished by tub2 and gapdh sequence data (Weir et al. 2012).

Colletotrichum tropicale E.I. Rojas, S.A. Rehner \& Samuels, Mycologia 102(6): 1331 (2010)

Sexual morph not reported. See Rojas et al. (2010) for illustrations and descriptions of asexual morph.

Type - Panama, Escobal, Chiriqui, on Annona muricata fruit rot, E.I. Rojas, neotype CBS 124945, ex-neotype culture CBS 124943.

Hosts - wide host range (See Farr \& Rossman 2021)

Distribution - tropical region (See Farr \& Rossman 2021)

Notes - It is a well-known pathogen on tropical trees. Pathogenicity studies are available for this taxon. Colletotrichum tropicale is best distinguished using tub2, chs-1, gs, or sod2 sequence data (Weir et al. 2012).

Colletotrichum viniferum L.J. Peng, L. Cai, K.D. Hyde \& Z-Y. Ying, Mycoscience 54(1): 36 (2013)

Sexual morph not reported. See Peng et al. (2013) for illustrations and descriptions of asexual morph.

Type - China, Yunnan Province, Kunming City, Ala Town, Gaopo Village, on fruits of Vitis vinifera cv. 'Shuijing', 30 July 2008, L.J. Peng holotype GZAAS5H.08601, ex-holotype culture GZAAS5.08601. 2018)

Hosts - Vitis vinifera (Peng et al. 2013, Yan et al. 2015, Oo \& Oh 2017, Jayawardena et al.

Distribution - China (Peng et al. 2013, Yan et al. 2015, Jayawardena et al. 2018), South Korea (Oo \& Oh 2017)

Notes - Colletotrichum viniferum was introduced to accommodate a pathogen causing leaf spot and fruit rot of grapevine. This species appears to be host-specific.

Colletotrichum wuxiense Yu Chun Wang, X.C. Wang \& Y.J. Yang, in Wang et al., Scientific Reports 6(35287): 8 (2016)

See Fu et al. (2019) for illustrations and descriptions of sexual morph. See Wang et al. (2016) for illustrations and descriptions of asexual morph.

Type - China, Jiangsu Province, Wuxi City, from diseased leaves of Camellia sinensis, 20 Aug. 2014, Y.C. Wang, holotype HMAS 246948, culture ex-type CGMCC 3.17894.

Hosts - Camellia sinensis (Wang et al. 2016, Fu et al. 2019)

Distribution - China (Wang et al. 2016, Fu et al. 2019)

Notes - This species can be separated from other species in the gloeosporioides complex by concatenated apmat and gs sequence data (Wang et al. 2016). Pathogenicity data are available for this species. 
Colletotrichum xanthorrhoeae R.G. Shivas, Bathgate \& Podger, Mycol. Res. 102 (3): 280 (1998)

Sexual morph not reported. See Shivas et al. (1998) for illustrations and descriptions of asexual morph.

Type - Australia, Western Australia, Melville, on Xanthorrhoea preissii (leaf spots), F.D. Podger, Jan. 1994, holotype WAC 8358, ex-holotype culture ICMP 17903.

Hosts - Xanthorrhoea preissii (Weir et al. 2012)

Distribution - Australia (Weir et al. 2012)

Notes - This species is only recorded from Xanthorrhoea preissii and can be identified by ITS sequence data (Weir et al. 2012).

Colletotrichum yulongense C.L. Hou \& X.T. Liu, in Wang et al., Phytotaxa 394(4): 293 (2019)

Sexual morph not reported. See Wang et al. (2019) for illustrations and descriptions of asexual morph.

Type - China, Yunnan Province, Yulong County, Lijiang, from the leaves of Vaccinium dunalianum var. urophyllum, alt. ca. 2400 m, 20 July 2013, coll. C.L. Hou, holotype BJTC 193, culture ex-holotype CFCC 50818.

Hosts - Vaccinium dunalianum (Wang et al. 2019)

Distribution - China (Wang et al. 2019)

Notes - Colletotrichum yulongense is closely related with $C$. henanense and can be distinguished by any of the loci (ITS, gapdh, chs-1, act and tub2) used in Wang et al. (2019).

\section{Magnum Species Complex}

The magnum species complex includes $C$. magnum and seven closely related species (Damm et al. 2019). Except for C. brevisporum other species appear to be host-specific. However, for some of these species only a single strain is available (Damm et al. 2019). A combined multigene analysis of ITS, gapdh, chs-1, act and tub2 sequence data is given in Fig. 14, Table 13.

Colletotrichum brevisporum Noireung, Phouliv., L. Cai \& K.D. Hyde, Cryptog. Mycol. 33(3): 350 (2012)

Sexual morph not reported. See Noireung et al. (2012) for illustrations and descriptions of asexual morph.

Type - Thailand, Nakhon Si Thammarat Province, Thasala District, Walailak University, on Neoregelia sp., 17 January 2008, Sitthisack Phoulivong holotype MFLU 110011, culture ex-type MFLUCC 110115.

Hosts - Anthurium sp., Carica papaya, Neoregalia sp., Pandanus pygmaeus, Passiflora edulis (Noireung et al. 2012, Damm et al. 2019)

Distribution - Australia, Japan, Thailand (Noireung et al. 2012, Damm et al. 2019)

Notes - Colletotrichum brevisporum can be identified based on its gapdh sequence data. This taxon is recorded as a pathogen and an endophyte. Pathogenicity studies are available for this species.

Colletotrichum cacao Damm, in Damm et al., Stud. Mycol. 92: 8 (2018)

Sexual morph not reported. See Damm et al. (2019) for illustrations and descriptions of asexual morph.

Type - Costa Rica, endophyte of Theobroma cacao, collection date and collector unknown, holotype CBS H-21068, culture ex-type CBS 119297.

Hosts - Theobroma cacao (Damm et al. 2019)

Distribution - Costa Rica (Damm et al. 2019)

Notes - The formation of setae that function as conidiogenous loci and conidia in closed conidiomata can differentiate this species from others in the complex (Damm et al. 2019). Colletotrichum cacao can be phylogenetically distinguished by all the loci used in (ITS, gapdh, chs-1, his3, act and tub2) Damm et al. (2019). 
Colletotrichum liaoningense Y.Z. Diao, C. Zhang, L. Cai \& Xi L. Liu, in Diao et al., Persoonia 38: 34 (2017) morph.

Sexual morph not reported. See Diao et al. (2017) for illustrations and descriptions of asexual

Type - China, Liaoning Province, Xingcheng city on fruits of Capsicum annuum var. conoides, Oct. 2012, Y.Z. Diao, holotype HMAS 246479, culture ex-type CGMCC3.17616.

Hosts - Capsicum annuum (Diao et al. 2017)

Distribution - China (Diao et al. 2017)

Notes - The four strains assigned to this species by Diao et al. (2017) need further clarifications as they formed two separate clades in Damm et al. (2019). Colletotrichum liaoningense is recorded only from Capsicum annuum and seems to be restricted to China. Pathogenicity studies for this species are available.

Colletotrichum lobatum Damm, in Damm et al., Stud. Mycol. 92: 16 (2018)

Sexual morph not reported. See Damm et al. (2019) for illustrations and descriptions of asexual morph.

Type - Trinidad and Tobago, from Piper catalpaefolium, collection date and collector unknown, holotype IMI 79736 holotype, culture ex-holotype IMI 79736.

Hosts - Piper catalpaefolium (Damm et al. 2019), Phaseolus lunatus (Cavalcante et al. 2019)

Distribution - Trinidad and Tobago (Damm et al. 2019), Brazil (Cavalcante et al. 2019)

Notes - Colletotrichum lobatum has lobate appressoria and can be identified by its unique gapdh sequence data (Damm et al. 2019). Pathogenicity studies for this taxon are available (Cavalcante et al. 2019). This species thought to be host-specific by the time it was introduced, however new collections show that it is not.

Colletotrichum merremiae Damm, in Damm et al., Stud. Mycol. 92: 21 (2018)

Sexual morph not reported. See Damm et al. (2019) for illustrations and descriptions of asexual morph.

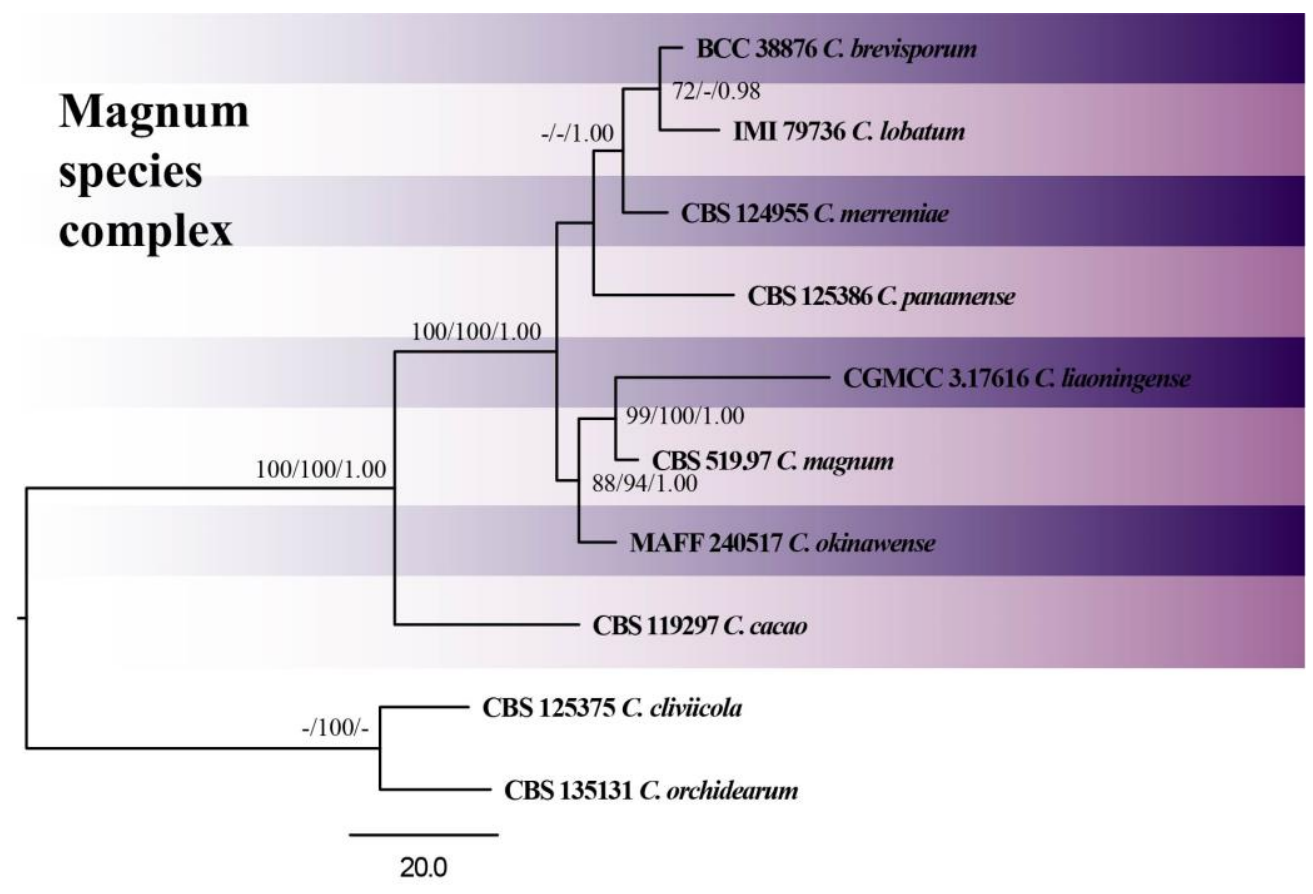

Figure 14 - The magnum complex. One of the 100 most parsimonious trees obtained from a heuristic search of combined ITS, gapdh, chs-1, act and tub2 sequence data. The MP and ML bootstrap support values $\geq 50 \%$ and BYPP $\geq 0.90$ are indicated at the nodes and branches. The tree is rooted with $C$. cliviicola (CBS 125375) and C. orchidearum (CBS 135131). 
Table 13 GenBank accession numbers of species in the magnum complex

\begin{tabular}{|c|c|c|c|c|c|c|c|}
\hline Species name & Type/Reference strain & ITS & gapdh & chs -1 & act & tub2 & References \\
\hline C. brevisporum & BCC 38876 & JN050238 & JN050227 & & JN050216 & JN050244 & Damm et al. (2019) \\
\hline C. cacao & CBS 119297 & MG600772 & MG600832 & MG600878 & MG600976 & MG601039 & Damm et al. (2019) \\
\hline C. liaoningense & CGMCC 3.17616 & KP890104 & KP890135 & KP890127 & KP890097 & KP890111 & Damm et al. (2019) \\
\hline C. lobatum & IMI 79736 & MG600768 & MG600828 & MG600874 & MG600972 & MG601035 & Damm et al. (2019) \\
\hline C. magnum & CBS 519.97 & MG600769 & MG600829 & MG600875 & MG600973 & MG601036 & Damm et al. (2019) \\
\hline C. merremiae & CBS 124955 & MG600765 & MG600825 & MG600872 & MG600969 & MG601032 & Damm et al. (2019) \\
\hline C. okinawense & MAFF 240517 & MG600767 & MG600827 & & MG600971 & MG601034 & Damm et al. (2019) \\
\hline C. panamense & CBS 125386 & MG600766 & MG600826 & MG600873 & MG600970 & MG601033 & Damm et al. (2019) \\
\hline
\end{tabular}

Type - Panama, Gamboa, wet lowland forest, leaf endophyte of Merremia umbellata, Nov. 2004, S. VanBael \& Z. Maynard, D3-1, holotype CBS H-21065, culture ex-type CBS 124955.

Hosts - Merremia umbellate (Damm et al. 2019)

Distribution - Panama (Damm et al. 2019)

Notes - Colletotrichum merremiae can be identified based on its unique gapdh, his3 and tub2 sequence data (Damm et al. 2019).

Colletotrichum magnum (S.F. Jenkins \& Winstead) Rossman \& W.C. Allen, in Rossman et al., IMA Fungus 7(1): 4 (2016)

See Damm et al. (2019) for illustrations and descriptions of asexual and sexual morphs.

Type - USA, from Citrullus lanatus, collection date and collector unknown, epitype CBS H-21063, culture ex-epitype CBS 519.97.

Hosts - Citrullus lanatus (Damm et al. 2019)

Distribution - USA (Damm et al. 2019)

Notes - As a result of a laboratory crossing, a heterothallic sexual morph for this species is known (Damm et al. 2019). Colletotrichum magnum is difficult to differentiate based on sequence data. This species was the basis and used as a model species for a number of molecular, morphological and pathogenicity studies on appressorium formation, pathogenic and symbiotic lifestyles of fungi in plants.

Colletotrichum okinawense Damm \& Toy. Sato, in Damm et al., Stud. Mycol. 92: 23 (2018)

Sexual morph not reported. See Damm et al. (2019) for illustrations and descriptions of asexual morph.

Type - Japan, Okinawa prefecture, Miyakojima Island, from a petiole of Carica papaya, 28 Sep. 2007, S. Sato, holotype GLM-F 111630, culture ex-holotype MAFF 240517.

Hosts - Carica papaya (Damm et al. 2019, Dias et al. 2020)

Distribution - Japan (Damm et al. 2019), Brazil (Dias et al. 2020)

Notes - Colletotrichum okinawense differ from all other species in magnum species complex gapdh, act and tub2 sequence data. This species produces predominantly clavate conidia, microcyclic conidiation and is the only fastest growing species in the complex. 
Colletotrichum panamense Damm, in Damm et al., Stud. Mycol. 92: 28 (2018)

Sexual morph not reported. See Damm et al. (2019) for illustrations and descriptions of asexual morph.

Type - Panama, Gamboa, wet lowland forest, leaf endophyte of Merremia umbellata, Nov. 2004, S. Van Bael \& Z. Maynard, D2-13, holotype CBS H-21066, culture ex-type CBS 125386.

Hosts - Merremia umbellate (Damm et al. 2019)

Distribution - Panama (Damm et al. 2019)

Notes - Colletotrichum panamense differs with all loci (ITS, gapdh, chs-1, his3, act and tub2) studied in Damm et al. (2019) from all other species of the genus. It is closely related to $C$. merremiae. However, the conidiogenous cells of $C$. panamense are often subglobose to ellipsoidal and can be differentiated from C. merremiae.

\section{Orbiculare species complex}

The orbiculare species complex includes $C$. orbiculare and seven closely related species. They are plant pathogens and are restricted to specific herbaceous host genera or species (Damm et al. 2013). The lifestyle of these species has been characterized as hemibiotrophic (Damm et al. 2013). Members of the orbiculare species complex form conidia that are straight and relatively broad and short. Appressoria of these species are small and simple in outline (Damm et al. 2013). All species of this species are either host-specific or geographically restricted. However, for some of these species only a single strain is available (Damm et al. 2013). All species in this complex can be identified based on $g s$ sequences alone (Damm et al. 2013). A combined multigene analysis of ITS, gapdh, chs-1, act and tub2 sequence data is given in Fig. 15, Table 14.

Colletotrichum bidentis Damm, Guatim. \& B.S. Vieira, in Damm et al., Fungal Diversity 61: 34 (2013)

Sexual morph not reported. See Damm et al. (2013) for illustrations and descriptions of asexual morph.

Type - Brazil, Goiás, Jataí, road to Rio Verde, abandoned gas station, from anthracnose symptoms on stems of Bidens subalternans, 13 Feb. 2010, B.S. Vieira, holotype VIC 31566, culture ex-holotype COAD 1020.

Hosts - Bidens subalternans (Damm et al. 2013)

Distribution - Brazil (Damm et al. 2013)

Notes - Colletotrichum bidentis is similar to $C$. lindemuthianum but can be separated from its setae and slow growing nature on medium (Damm et al. 2013). Even though this species has been isolated disease symptoms materials, no pathogenicity data are available to confirm it. This taxon is separable from all other species in this complex by any of the loci (ITS, gapdh, chs-1, his3, act, tub2 and $g s$ ) used in Damm et al. (2013). There are no records of this species other than that of the type.

Colletotrichum lindemuthianum (Sacc. \& Magnus) Briosi \& Cavara, Funghi Parass. Piante Colt. od Utili, Fasc. 2: no. 50 (1889)

Sexual morph not reported. See Liu et al. (2013) for illustrations and descriptions of asexual morph.

Type - Germany, Bonn. on Phaseolus vulgaris, Aug 1931, E. Schaffnit epitype CBS H20954, culture ex-epitype CBS 144.31.

Hosts - Phaseolus coccineus, P. vulgaris (Liu et al. 2013b)

Distribution - Worldwide (Jayawardena et al. 2016a)

Notes - This species is restricted to Phaseolus sp. with a worldwide distribution. Colletotrichum lindemuthianum is used as a model organism in understanding the hemi-biotrophic nature of this genus (Cannon et al. 2012, Jayawardena et al. 2016a).

Colletotrichum malvarum (A. Braun \& Casp.) Southw., J. Mycol. 6(3): 116 (1891) 
Sexual morph not reported. See Damm et al. (2013) for illustrations and descriptions of asexual morph.

Type - UK, from Lavatera trimestris cv. 'Mont Blanc', unknown collection date, R. Maude, epitype CBS H-20973, culture ex-epitype CBS 521.97.

Hosts - genera of Malvaceae (Damm et al. 2013)

Distribution - Germany, Korea, UK (Damm et al. 2013)

Notes - It is a restricted pathogen causing anthracnose and leaf spots on genera of Malvaceae (Damm et al. 2013). Colletotrichum malvarum is one of the slowest growing species in the orbiculare complex (except for $C$. bidentis). This taxon is closely related to $C$. trifolii and can be distinguished by $g s$ sequence data.

Colletotrichum orbiculare Damm, P.F. Cannon \& Crous, in Damm et al., Fungal Diversity 61: 39 (2013)

Sexual morph not reported. See Damm et al. (2013) for illustrations and descriptions of asexual morph.

Type - Unknown country in Europe (probably UK), from Cucumis sativus, collection date unknown, G.A. Carter, holotype CBSH-20976, culture ex-holotype CBS 570.97.

Hosts - genera of Cucubitaceae (Damm et al. 2013)

Distribution - Australia, Japan, Netherland, UK (Damm et al. 2013)

Notes - Colletotrichum orbiculare may have a wide distribution as there are many records in US national fungus collections (Farr \& Rossman 2021). As most of those records are based on morphology alone, further collections are needed to confirm these. This taxon has been used as a model in host-pathogen interactions in Colletotrichum (Jayawardena et al. 2016a). This species can be separated from other species with its unique gs, act and his3 sequence data (Damm et al. 2013). Whole genomic data for this species are available (Baroncelli et al. 2016).

Colletotrichum sidae Damm \& P.F. Cannon, in Damm et al., Fungal Diversity 61: 44 (2013)

Sexual morph not reported. See Damm et al. (2013) for illustrations and descriptions of asexual morph.

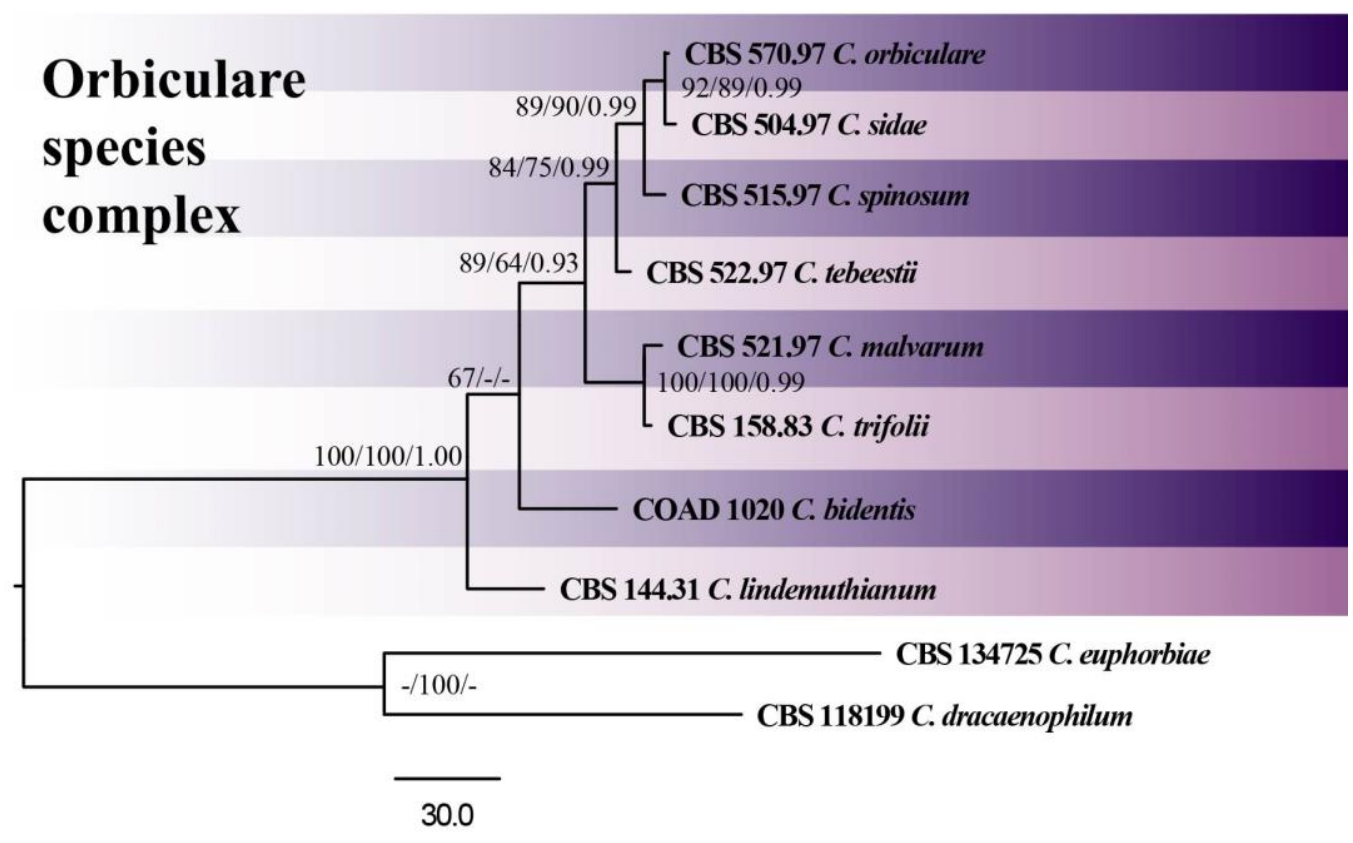

Figure 15 - Orbiculare complex. One of the 100 most parsimonious trees obtained from a heuristic search of combined ITS, gapdh, chs-1, act and tub2 sequence data. The MP and ML bootstrap support values $\geq 50 \%$ and $\mathrm{BYPP} \geq 0.90$ are indicated at the nodes and branches. The tree is rooted with C. euphorbiae (CBS 134725) and C. dracaenophilum (CBS 118199). 
Table 14 GenBank accession numbers of species in the orbiculare complex

\begin{tabular}{|c|c|c|c|c|c|c|c|}
\hline Species name & Type/Reference strain & ITS & gapdh & chs-1 & act & tub2 & References \\
\hline C. bidentis & COAD 1020 & KF178481 & KF178506 & KF178530 & KF178578 & KF178602 & Damm et al. (2013) \\
\hline C. lindemuthianum & CBS 144.31 & JQ005779 & JX546712 & JQ005800 & JQ005842 & JQ005863 & Damm et al. (2013) \\
\hline C. malvarum & CBS 521.97 & KF178480 & KF178504 & KF178529 & KF178577 & KF178601 & Damm et al. (2013) \\
\hline C. orbiculare & CBS 570.97 & KF178466 & KF178490 & KF178515 & KF178563 & KF178587 & Damm et al. (2013) \\
\hline C. sidae & CBS 504.97 & KF178472 & KF178497 & KF178521 & KF178569 & KF178593 & Damm et al. (2013) \\
\hline C. spinosum & CBS 515.97 & KF178474 & KF178498 & KF178523 & KF178571 & KF178595 & Damm et al. (2013) \\
\hline C. tebeesti & CBS 522.97 & KF178473 & KF178505 & KF178522 & KF178570 & KF178594 & Damm et al. (2013) \\
\hline C. trifolii & CBS 158.83 & KF178478 & KF178502 & KF178527 & KF178575 & KF178599 & Damm et al. (2013) \\
\hline
\end{tabular}

Type - USA, Arkansas, from Sida spinosa, 1 May 1988, D.O. TeBeest, holotype CBS H-20975, culture ex-holotype CBS 504.97.

Hosts - Sida spinosa (Damm et al. 2013)

Distribution - USA (Damm et al. 2013)

Notes - Colletotrichum sidae can be separated from its closely related taxa from ITS and $g s$ sequence data (Damm et al. 2013).

Colletotrichum spinosum Damm \& P.F. Cannon, in Damm et al., Fungal Diversity 61: 46 (2013)

Sexual morph not reported. See Damm et al. (2013) for illustrations and descriptions of asexual morph.

Type - Australia, New South Wales, Coolah, from stem lesion of Xanthium spinosum, 20 Mar. 1983, B. Auld, holotype CBS H-20977, culture ex-holotype CBS 515.97.

Hosts - Xanthium spinosum (Damm et al. 2013)

Distribution - Argentina, Australia (Damm et al. 2013)

Notes - Colletotrichum spinosum is a common pathogen (stem and leaf spots) on Xanthium spinosum in Australia (Walker et al. 1991), and has been tested as a mycoherbicide against this weed (Auld \& Say 1999). This taxon can be effectively separated from the related taxa based on gapdh, his3, tub2 and $g s$ sequence data (Damm et al. 2013).

Colletotrichum tebeestii Damm \& P.F. Cannon, in Damm et al., Fungal Diversity 61: 48 (2013)

Sexual morph not reported. See Damm et al. (2013) for illustrations and descriptions of asexual morph.

Type - Canada, Saskatchewan, Raymore, from Malva pusilla, unknown collection date, D.O. TeBeest, holotype CBS H-20974, culture exholotype CBS 522.97.

Hosts - Malva pusilla (Damm et al. 2013)

Distribution - Canada (Damm et al. 2013) 2013).

Notes - Colletotrichum tebeestii can best be differentiated from other species in the complex by its unique gapdh sequence data (Damm et al. 
Colletotrichum trifolii Bain, in Bain \& Essary, J. Mycol. 12(5): 193 (1906)

Sexual morph not reported. See Damm et al. (2013) for illustrations and descriptions of asexual morph.

Type - USA, from Trifolium sp., collection date and collector unknown, epitype CBS H20978, culture ex-epitype CBS 158.83.

Hosts - Medicago sp., Trifolium sp. (Damm et al. 2013)

Distribution - USA (Damm et al. 2013)

Notes - This species has darker and often knobby setae than the other species in this complex (Damm et al. 2013). Colletotrichum trifolli is closely related to C. malvarum and can be separated best with gs sequence data. In Farr \& Rossman (2021) there are more host associations for this taxon. However, as they are based solely on morphology further collections are needed to clarify.

\section{Orchidearum species complex}

The orchidearum species complex includes $C$. orchidearum and seven closely related species (Damm et al. 2019). Three of the species (C. orchidearum, C. plurivorum, C. sojae) are very common and occur on many hosts while the rest are less common $(C$. cliviicola, $C$. musicola $)$ and some being host specific (C. cattleyicola) and restricted to a specific region $(C$. piperis, $C$. vittalense). In the study of Damm et al. (2019), sexual morphs were observed for many of the species in this complex. Therefore, they assumed them to be homothallic, however, this is yet to be confirmed. A combined multigene analysis of ITS, gapdh, chs-1, act and tub2 sequence data is given in Fig. 16, Table 15.

Colletotrichum cattleyicola Damm \& Toy. Sato, in Damm et al., Stud. Mycol. 92: 10 (2018)

Sexual morph not reported. See Damm et al. (2019) for illustrations and descriptions of asexual morph.

Type - Belgium, from a root of Cattleya sp., collection date and collector unknown, holotype CBS H-21502 holotype, culture ex-holotype CBS 170.49 .

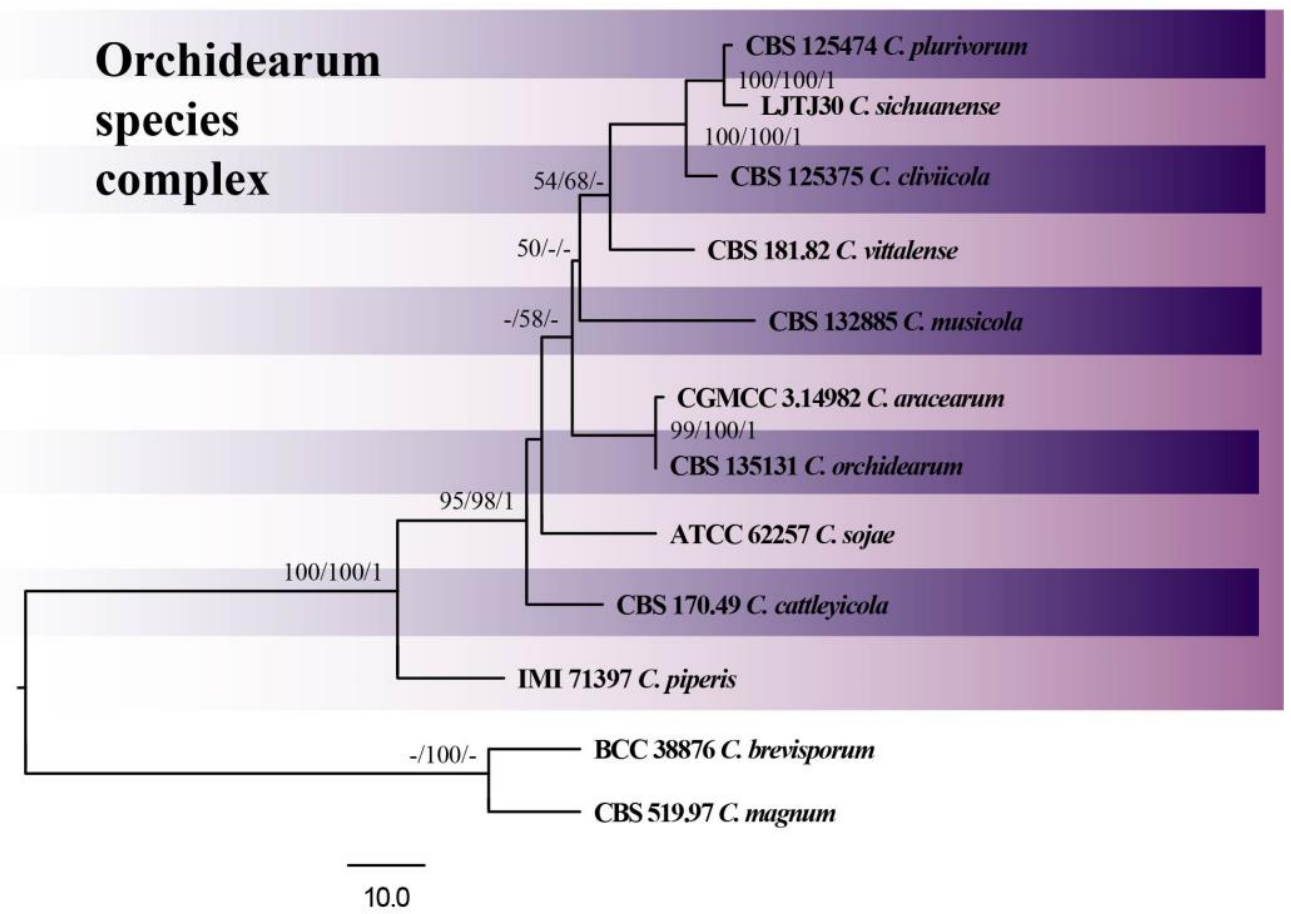

Figure 16 - The orchidearum complex. One of the 100 most parsimonious trees obtained from a heuristic search of combined ITS, gapdh, chs-1, act and tub2 sequence data. The MP and ML bootstrap support values $\geq 50 \%$ and BYPP $\geq 0.90$ are indicated at the nodes and branches. The tree is rooted with C. brevisporum (BCC 38876) and C. magnum (CBS 519.97). 
Table 15 GenBank accession numbers of species in the orchidearum complex

\begin{tabular}{|c|c|c|c|c|c|c|c|}
\hline Species name & Type/Reference strain & ITS & gapdh & chs-1 & act & tub2 & References \\
\hline C. cattleyicola & CBS 170.49 & MG600758 & MG600819 & MG600866 & MG600963 & MG601025 & Damm et al. (2019) \\
\hline C. cliviicola & CBS 125375 & MG600733 & MG600795 & MG600850 & MG600939 & MG601000 & Damm et al. (2019) \\
\hline C. musicola & CBS 132885 & MG600736 & MG600798 & MG600853 & MG600942 & MG601003 & Damm et al. (2019) \\
\hline C. orchidearum & CBS 135131 & MG600738 & MG600800 & MG600855 & MG600944 & MG601005 & Damm et al. (2019) \\
\hline C. piperis & IMI 71397 & MG600760 & MG600820 & MG600867 & MG600964 & MG601027 & Damm et al. (2019) \\
\hline C. plurivorum & CBS 125474 & MG600718 & MG600781 & MG600841 & MG600925 & MG600985 & Damm et al. (2019) \\
\hline C. sojae & ATCC 62257 & MG600749 & MG600810 & MG600860 & MG600954 & MG601016 & Damm et al. (2019) \\
\hline C. vittalense & CBS 181.82 & MG600734 & MG600796 & MG600851 & MG600940 & MG601001 & Damm et al. (2019) \\
\hline
\end{tabular}

Hosts - Cattleya sp. (Damm et al. 2019)

Distribution - Belgium, Japan (Damm et al. 2019)

Notes - This taxon can be differentiated by its unique appressoria (narrow, mostly clavate or elongate cylindrical) from other species in this complex. Phylogenetically it can be distinguished by ITS, his3 and tub2 sequence data (Damm et al. 2019). This species has been isolated from root and lesion on a stem sheath of Cattleya sp.

Colletotrichum cliviicola Damm \& Crous, in Damm et al., Stud. Mycol. 92: 11 (2018)

Sexual morph not reported. See Damm et al. (2019) for illustrations and descriptions of asexual morph.

Type - China, Yunnan Province, Kunming, on leaf of Clivia miniata, 10 Aug. 2008, Y.L. Yang, holotype GZAAS 080005, culture ex-holotype CBS 125375 .

Hosts - Clivia sp. (Damm et al. 2019), Pennisetum sp. (Han et al. 2019)

Distribution - China (Damm et al. 2019, Han et al. 2019), South Africa (Damm et al. 2019)

Notes - Colletotrichum cliviicola differs from the closely related C. plurivorum in its tub2, his3 and gapdh sequence data (Damm et al. 2019). Pathogenicity studies for this taxon are available (Han et al. 2019).

Colletotrichum musicola Damm, in Damm et al., Stud. Mycol. 92: 21 (2018)

See Damm et al. (2019) for illustrations and descriptions of asexual and sexual morphs.

Type - Mexico, from Musa sp., 16 Dec. 2008, M. de J. Yanez Morales holotype CBS H-21500, culture ex-holotype CBS 132885.

Hosts - Musa sp. (Damm et al. 2019), Colocasia esculenta (Vasquez-Lopez et al. 2019), Phaseolus lunatus (Cavalcante et al. 2019), Glyzine $\max$ (Boufleur et al. 2020)

Distribution - Mexico (Damm et al. 2019, Vasquez-Lopez et al. 2019), Brazil (Cavalcante et al. 2019, Boufleur et al. 2020) 
Notes - Colletotrichum musicola can be identified with all loci (ITS, gapdh, chs-1, his3, act and tub2) studied in Damm et al. (2019). The species was introduced from Musa sp. from Mexico. Later it was found to be a pathogen on Glyzine max and Phaseolus lunatus in Brazil. Pathogenicity studies for this taxon are available.

Colletotrichum orchidearum Allesch., Rabenh. Krypt.-Fl., Edn 2 (Leipzig) 1(7): 563 (1902) [1903]

See Damm et al. (2019) for illustrations and descriptions of asexual and sexual morphs.

Type - Netherlands, Utrecht, from anthracnose on leaf of Dendrobium nobile, Apr. 2013, I. Benoit-Gelber, epitype CBS H-21910, culture ex-epitype CBS 135131.

Hosts - Cordyline, Hymenocallis, Monstera, Philodendron, Scindapus, Orchidaceae (Cattleya, Cymbidium, Dendrobium, Oncidium, Phalaenopsis and Vanda) Damm et al. (2019)

Distribution - China, Iran, Japan, Thailand, Netherland (Damm et al. 2019)

Notes - Colletotrichum orchidearum can be differentiated with sequence data of all loci (ITS, chs-1, his3, act and tub2) included in Damm et al. (2019), except for gapdh. Pathogenicity studies are available for this taxon.

Colletotrichum piperis Petch, Ann. R. bot. Gdns Peradeniya 6(3): 239 (1917)

Sexual morph not reported. See Damm et al. (2019) for illustrations and descriptions of asexual morph.

Type - Malaysia, from Piper nigrum, unknown collection date and collector epitype IMI 71397, culture ex-epitype IMI 71397.

Hosts - Piper nigrum (Damm et al. 2019)

Distribution - Malaysia (Damm et al. 2019)

Notes - Colletotrichum piperis can be identified with all loci (ITS, gapdh, chs-1, his3, act and tub2) in Damm et al. (2019).

Colletotrichum plurivorum Damm, Alizadeh \& Toy. Sato, in Damm et al., Stud. Mycol. 92: 31 (2018)

See Damm et al. (2019) for illustrations and descriptions of asexual and sexual morphs.

Type - Vietnam, Da Lat-Lam Dong, from anthracnose on leaf of Coffea sp., collection date unknown, P. Nguyen \& E. Liljeroth, holotype CBS H-21496, culture ex-holotype CBS 125474.

Hosts - Abelmoschus esculentus, Amorphophallus rivieri, Arundina graminifolia, Camellia sinensis, Capsicum annuum, Carica papaya, Coffea sp., Cymbidium hookerianum, Glycine max, Gossypium sp., Lycopersicon esculentum, Mangifera indica, Musa sp., Oncidium sp., Passiflora edulis, Phaseolus sp., Spathiphyllum wallisii (Damm et al. 2019), Pyrus bretschneideri (Fu et al. 2019)

Distribution - Benin, Brazil, China, Iran, Japan, Vietnam (Damm et al. 2019)

Notes - Colletotrichum plurivorum was described as C. sichuanensis from Capsicum annuum in the Sichuan Province of China (Liu et al. 2016a) and was invalidly published. This taxon has a wide host range. Pathogenicity studies for this taxon are available. Phylogenetically this species can be distinguished by its gapdh, his 3 and tub2 sequence data from its sister taxon, C. cliviicola. These two taxa can be distinguished by the presence of microcyclic conidiation and the formation of anastomoses in C. cliviicola (Damm et al. 2019)

Colletotrichum sojae Damm \& Alizadeh, in Damm et al., Stud. Mycol. 92: 35 (2018)

See (Damm et al. 2019) for illustrations and descriptions of asexual and sexual morphs.

Type - USA, North Carolina, Raleigh, from anthracnose of Glycine max, unknown collection date and collector, holotype BPI 596658 (dried culture), culture ex-holotype CBS 195.32.

Hosts - Arctium lappa, Bletilla ochracea, Capsicum sp., Glycine max, Medicago sativa, Phaseolus vulgaris, Vigna unguiculata (Damm et al. 2019)

Distribution - Brazil, China, Iran, Italy, Serbia, USA (Damm et al. 2019) 
Notes - Colletotrichum sojae can be identified by its his3, act and tub2 sequences data (Damm et al. 2019). There are no pathogenicity studies available for this species.

Colletotrichum vittalense Damm, in Damm et al., Stud. Mycol. 92: 38 (2018)

See (Damm et al. 2019) for illustrations and descriptions of asexual and sexual morphs.

Type - India, Karnataka, Vittal, from Theobroma cacao, collection date and collector unknown, holotype CBS H-21498, culture ex-holotype CBS 181.82.

Hosts - Calamus thwaitesii, Orchid sp., Theobroma cacao (Damm et al. 2019)

Distribution - India (Damm et al. 2019)

Notes - Colletotrichum vittalense can be distinguished by its very short ascospores with round ends that vary between straight to strongly curved. This taxon can be identified by its unique gapdh, chs-1, his3 and tub2 sequence data (Damm et al. 2019). Even though the species was described as a pathogen there are no pathogenicity studies to confirm this.

\section{Spaethianum species complex}

The spaethianum species complex includes $C$. spaethianum and eight closely related species. Species in this species complex form complex appressoria (Damm et al. 2009). A combined multigene analysis of ITS, gapdh, chs-1, act, his3 and tub2 sequence data is given in Fig. 17, Table 16.

Colletotrichum bletillae G. Tao, Zuo Y. Liu \& L. Cai [as 'bletillum'], in Tao et al., Fungal Diversity 61: 144 (2013)

Sexual morph not reported. See Tao et al. (2013) for illustrations and descriptions of asexual morph.

Type - China, Guizhou Province, Shuicheng, Baijipo Mountain, isolated from healthy leaves of Bletilla ochracea, 28 June 2006, Gang Tao, holotype HMAS 244278 (dried culture), culture exholotype CGMCC 3.15117.

Hosts - Bletilla ochracea (Tao et al. 2013)

Distribution - China (Tao et al. 2013)

Notes - Colletotrichum bletillum was isolated as an endophyte and phylogenetically can be distinguished by any of the loci (ITS, act, gapdh and tub2) used in Tao et al. (2013).

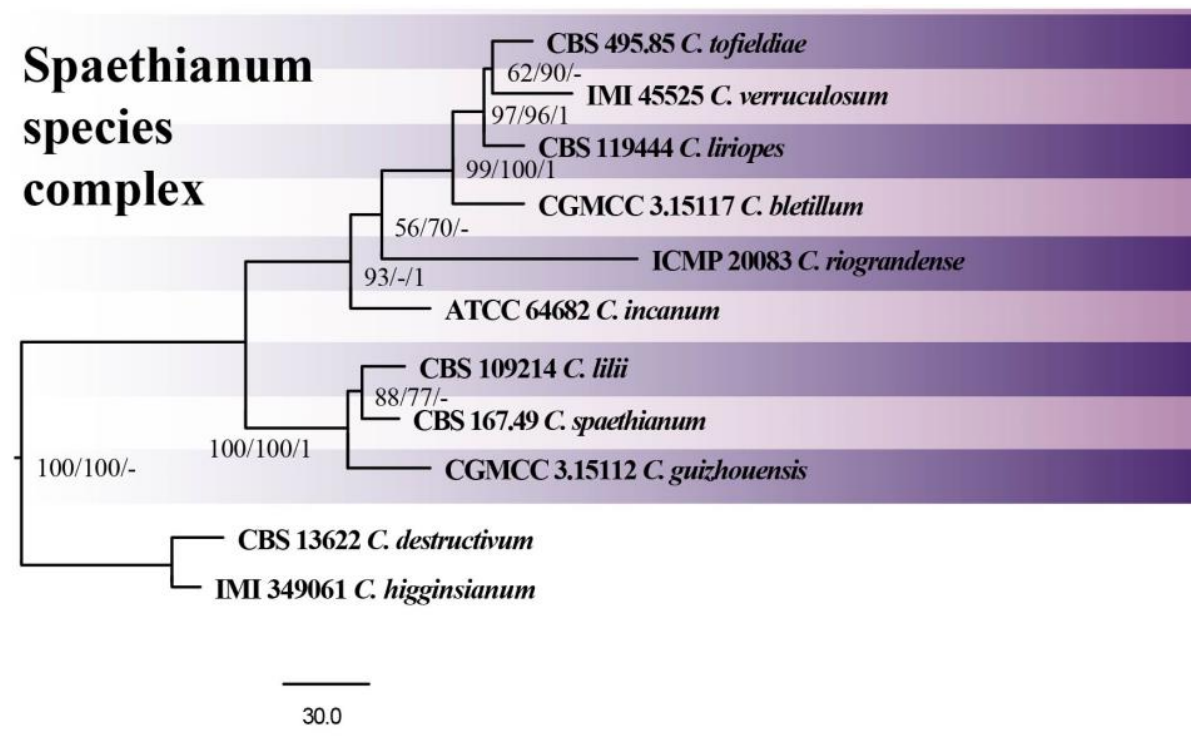

Figure 17 The spaethianum complex. One of the 100 most parsimonious trees obtained from a heuristic search of combined ITS, gapdh, chs-1, act and tub2 sequence data. The MP and ML bootstrap support values $\geq 50 \%$ and $\mathrm{BYPP} \geq 0.90$ are indicated at the nodes and branches. The tree is rooted with $C$. destructivum (CBS 13622) and C. higginsianum (IMI 349061). 
Table 16 GenBank accession numbers of species in the spaethianum complex

\begin{tabular}{|c|c|c|c|c|c|c|c|}
\hline Species name & Type/Reference strain & ITS & gapdh & chs-1 & act & $t u b 2$ & References \\
\hline C. bletillum & CGMCC 3.15117 & JX625178 & KC843506 & & KC843542 & JX625207 & Tao et al. (2013) \\
\hline C. guizhouensis & CGMCC 3.15112 & JX625158 & KC843536 & & KC843536 & JX625185 & Tao et al. (2013) \\
\hline C.incanum & ATCC 64682 & KC110789 & KC110807 & & KC110825 & KC110816 & Yang et al. (2014) \\
\hline C. lilii & CBS 109214 & GU227810 & GU228202 & GU228300 & GU227908 & GU228104 & Damm et al. (2009) \\
\hline C. liriopes & CBS 119444 & GU227804 & GU228196 & GU228294 & GU227902 & GU228098 & Damm et al. (2009) \\
\hline C. riograndense & ICMP 20083 & KM655299 & KM655298 & KM655297 & KM655295 & KM655300 & Macedo et al. (2016) \\
\hline C. spaethianum & CBS 167.49 & GU227807 & GU228199 & GU228297 & GU227905 & GU228101 & Damm et al. (2009) \\
\hline C. tofieldiae & CBS 495.85 & GU227801 & GU228193 & GU228291 & GU227899 & GU228095 & Damm et al. (2009) \\
\hline C. verruculosum & IMI 45525 & GU227806 & GU228198 & GU228296 & GU227904 & GU228100 & Damm et al. (2009) \\
\hline
\end{tabular}

Colletotrichum guizhouensis G. Tao, Zuo Y. Liu \& L. Cai, in Tao, Liu, Liu, Gao \& Cai, Fungal Diversity 61: 152 (2013)

Sexual morph not reported. See Tao et al. (2013) for illustrations and descriptions of asexual morph.

Type - China, Guizhou Province, Duyun, Xiaba mountain, isolated from healthy leaves of Bletilla ochracea, 13 July 2006, Gang Tao, Holotype HMAS244281 (dried culture), culture ex-holotype CGMCC 3.15112.

Hosts - Bletilla ochracea (Tao et al. 2013)

Distribution - China (Tao et al. 2013)

Notes - This taxon is an endophyte. Colletotrichum guizhouensis is similar to $C$. lilii and $C$. spaethianum but differs from them in having darker and longer setae (Tao et al. 2013). Zhang et al. (2015) showed that this species is capable of producing Huperzine A.

Colletotrichum incanum H.C. Yang, J.S. Haudenshield \& G.L. Hartman, Mycologia 106(1): 38 (2014)

Sexual morph not reported. See Yang et al. (2014) for illustrations and descriptions of asexual morph.

Type - USA, Illinois, Monmouth, isolated from diseased soybean (Glycine max) petioles collected from the soybean field, 14 Jun 2010 , H.-C. Yang, J.S. Haudenshield \& G.L. Hartman, holotype BPI884116, cultures ex-holotype (IL6A 5) CBS 133485.

Hosts - Glycine $\max$ (Yang et al. 2014)

Distribution - USA (Yang et al. 2014)

Notes - Sequence data of $a c t, t u b 2$, gapdh and his 3 genes can be used to distinguish this taxon from other species in this complex. Whole genomic data for this species are available (Gan et al. 2016).

Colletotrichum lilii Plakidas ex Boerema \& Hamers, Neth. Jl Pl. Path. 94 (suppl.1): 12 (1988)

Sexual morph not reported. See Damm et al. (2009) for illustrations and descriptions of asexual morph.

Reference specimen - Japan, unlocalised, on Lilium sp., deposited in CBS collection Jan. 2001 by H. Nirenberg, herbarium CBS H-20361 (dried culture), living culture CBS 109214.

Hosts - Lillium sp. (Damm et al. 2009) 
Distribution - Japan (Damm et al. 2009) bulbs.

Notes - This species is recorded as a pathogen on Lillium sp. causing black scale disease of

Colletotrichum liriopes Damm, P.F. Cannon \& Crous, in Damm et al., Fungal Diversity 39: 71 (2009)

Sexual morph not reported. See Damm et al. (2009) for illustrations and descriptions of asexual morph.

Type - Mexico, Aphis interception Houstan 057263, on Liriope muscari, collected 29 Nov. 2000 by M.J. Segall, isolated 2000 by A.Y. Rossman, holotype CBS H-20364, culture ex-type CBS 119444.

Hosts - Lirope muscari (Damm et al. 2009), Eria coronaria, Pleione bulbocodioides (Yang et al. 2011), Bletilla ochracea (Tao et al. 2013), Liriope spicata (Chen et al. 2019a), Liriope cymbidiomorpha (Yang et al. 2020)

Distribution - Mexico (Damm et al. 2009), China (Yang et al. 2011, Tao et al. 2013, Chen et al. 2019a, Yang et al. 2020)

Notes - This taxon can be distinguished by its appressoria with complex outlines that are similar to those of $C$. lilii. However, it differs from it by the often strongly inflated conidiogenous cells (Damm et al. 2009). Colletotrichum liriopes has been isolated as a pathogen as well as an endophyte. Pathogenicity studies for this species are available.

Colletotrichum riograndense D.M. Macedo, R.W. Barreto, O.L. Pereira \& B.S. Weir, in Macedo et al., Australasian Plant Pathology 45: 49 (2016)

Sexual morph not reported. See Macedo et al. (2016) for illustrations and descriptions of asexual morph.

Type - Brazil, Rio Grande do Sul, São Marcos, on living leaves of Tradescantia fluminensis, 13 Jul. 2008, D. M. Macedo, holotype VIC 31366, culture ex-type COAD 928.

Hosts - Tradescantia fluminensis (Macedo et al. 2016)

Distribution - Brazil (Macedo et al. 2016)

Notes - Colletotrichum riograndense is similar to C. falcatum (Macedo et al. 2016).

Colletotrichum spaethianum (Allesch.) Damm, P.F. Cannon \& Crous, in Damm et al., Fungal Diversity 39: 74 (2009)

Sexual morph not reported. See Damm et al. (2009) for illustrations and descriptions of asexual morph.

Type - Germany, Berlin-Zehlendorf, on a dead stem of Hosta sieboldiana, isolated Oct. 1932 by H. Richter, epitype CBS H-20369 (dried culture), culture ex-epitype CBS 167.49.

Hosts - Hosta sieboldiana, Lilium sp. (diseases leaves), Hemerocallis sp. (leaf spot) (Damm et al. 2009), Peucedanum praeruptorum (Guo et al. 2013), Allium sp. (Santana et al. 2016, Salunkhe et al. 2018b), Hosta plantaginea (Cheon \& Jeon 2016), Lilium lancifolium (Zhao et al. 2016b), Atractylodes japonica (Guan et al. 2018), Anemarrhena asphodeloides (Okorley et al. 2019), Phaseolus vulgaris (Yang et al. 2019a), Paris polyphylla (Zhong et al. 2020), Polygonatum odoratum (Liu et al. 2020b)

Distribution - Germany, New Zealand, South Korea (Damm et al. 2009), China (Guo et al. 2013, Zhao et al. 2016b, Guan et al. 2018, Okorley et al. 2019, Yang et al. 2019a, Liu et al. 2020b, Zhong et al. 2020), Brazil (Santana et al. 2016), Korea (Cheon \& Jeon 2016), India (Salunkhe et al. 2018b)

Notes - This taxon can be distinguished from related taxa with setae that have usually acute tips and cylindrical to conical bases and by its appressoria that are irregular in outline and more or less lobed (Damm et al. 2009). Pathogenicity studies for this species are available. 
Colletotrichum tofieldiae (Pat.) Damm, P.F. Cannon \& Crous, in Damm et al., Fungal Diversity 39: 77 (2009)

Sexual morph not reported. See Damm et al. (2009) for illustrations and descriptions of asexual morph.

Type - Switzerland, Graubünden, from Tofieldia calyculata, isolated July1985 by J.A. von Arx, holotype CBS H-20367 (dried culture), culture ex-type CBS 495.85.

Hosts - Dianthus sp., Lupinus polyphyllus, Tofieldia sp. (Damm et al. 2009), Bletilla ochracea (Tao et al. 2013), Arabidopsis thaliana (Hacquard et al. 2016), Iris $\times$ germanica (Shivas et al. 2016)

Distribution - Germany, Switzerland, UK (Damm et al. 2009), China (Tao et al. 2013), Australia (Shivas et al. 2016), Spain (Hiruma et al. 2016)

Notes - This taxon is recorded as a leaf and root endophyte (Tao et al. 2013, Hiruma et al. 2016). Pathogenicity of this species is unknown. Whole genomic data for this species are available (Hacquard et al. 2016).

Colletotrichum verruculosum Damm, P.F. Cannon \& Crous, in Damm et al., Fungal Diversity 39: 81 (2009)

Sexual morph not reported. See Damm et al. (2009) for illustrations and descriptions of asexual morph.

Type - Zimbabwe, Crotalaria juncea, 1951 holotype IMI 45525, culture ex-type IMI 45525.

Hosts - Crotalaria juncea (Damm et al. 2009)

Distribution - Zimbabwe (Damm et al. 2009)

Notes - Colletotrichum verruculosum can be distinguished by any of the six genes used by Damm et al. (2009). This species can be distinguished by its verruculose conidia. There are no pathogenicity studies on this species.

\section{Truncatum species complex}

The truncatum species complex includes $C$. truncatum and four closely related species. Among them three are pathogens and two are saprobes (Damm et al. 2009, Wikee et al. 2011, Ariyawansa et al. 2015, Samarakoon et al. 2018). This complex can be distinguished by their curved conidia with truncate base and acute, more strongly curved apex (Damm et al. 2009, Cannon et al. 2012, Jayawardena et al. 2016a). The presence of appressoria in groups and dense clades is also characteristic features of this complex (Damm et al. 2009, Jayawardena et al. 2016a). Other than $C$. truncatum, species of this complex seems to be host-specific. Colletotrichum fusiformae and C. truncatum have been identified as pathogens on humans (Hung et al. 2020). A combined analysis of ITS, gapdh, chs-1, act, his3 and tub2 sequence data is given in Fig. 18, Table 17.

Colletotrichum acidae Samarak. \& K.D. Hyde, in Samarakoon et al., Mycosphere 9(3): 587 (2018)

Sexual morph not reported. See Samarakoon et al. (2018) for illustrations and descriptions of asexual morph.

Type - Thailand, Mae Fah Luang University, Mueang, Chiang Rai on dead rachis of Phyllanthus acidus, 18 May 2017, Milan C. Samarakoon (SAMC004), holotype-MFLU 18-0100, culture ex-type MFLUCC 17-2659.

Hosts - Phyllanthus acidus (Samarakoon et al. 2018)

Distribution - Thailand (Samarakoon et al. 2018)

Notes - It is closely related to C. fusiforme. Colletotrichum acidae seems to be host specific and restricted to Thailand.

Colletotrichum curcumae (Syd. \& P. Syd.) E.J. Butler \& Bisby, Fungi of India: 153 (1931)

Sexual morph not reported. See Damm et al. (2009) for illustrations and descriptions of asexual morph. 
Epitype - India. Maharashtra, Warora, Curcuma longa, 22 Aug. 1984, M.Y. Palarpawar, epitype (dried culture) IMI288937, culture ex-epitype IMI 288937.

Hosts - Curcuma longa, Curcuma wenyujin (Damm et al. 2009)

Distribution - India (Damm et al. 2009), China (Li et al. 2016a)

Notes - Colletotrichum curcumae can be distinguished from the species with curved conidia by big brown flattened stromata with straight setae that are aggregated in the centre and with little sporulation (Damm et al. 2009). This species is only recorded from Curcuma as a pathogen. Pathogenicity studies for this species are available.

Colletotrichum fusiforme Jayawardena, Bhat, Tangthirasunun \& K.D. Hyde, in Ariyawansa et al., Fungal Diversity 75(1): 158 (2015)

Sexual morph not reported. See Ariyawansa et al. (2015) for illustrations and descriptions of asexual morph.

Type - Thailand, Chiang Rai, on a dead leaf, host unknown, 14 July 2012, J. Bhat NTCL98, holotype MFLU 13-0291, culture ex-type MFLUCC 12-0437.

Hosts - Homo sapiens

Distribution - Thailand (Ariyawansa et al. 2015), China (Hung et al. 2020)

Notes - This species was introduced as a saprobe from an undetermined host. Therefore, its host-specificity cannot be considered. So far this species has been recorded only from Thailand. There is a recent record of keratitis caused by this species in humans.

Colletotrichum jasminigenum Wikee, K.D. Hyde, L. Cai \& McKenzie, in Wikee et al., Fungal Diversity 46(1): 174 (2011)

Sexual morph not reported. See Wikee et al. (2011) for illustrations and descriptions of asexual morph.

Type - Vietnam, Ho Chi Min City, $12^{\text {th }}$ District, Thanh Xuan Ward, on living leaves of Jasminum sambac, May 2009, Hoa Nguyen Thi, LLTX-01, holotype MFLU 10-0212, ex-type culture MFLUCC 10-0273.

Hosts - Jasminum sambac (Wikee et al. 2011)

Distribution - Vietnam (Wikee et al. 2011)

Notes - Colletotrichum jasminigenum appears to be host specific and restricted to Vietnam. However, further studies are needed to confirm this. The sequence data of act and gapdh of this species seem to have a problem, as they show a higher similarity to $C$. siamense. Therefore, they were not included into our analyses. We strongly suggest redoing the sequencing for these two gene regions.

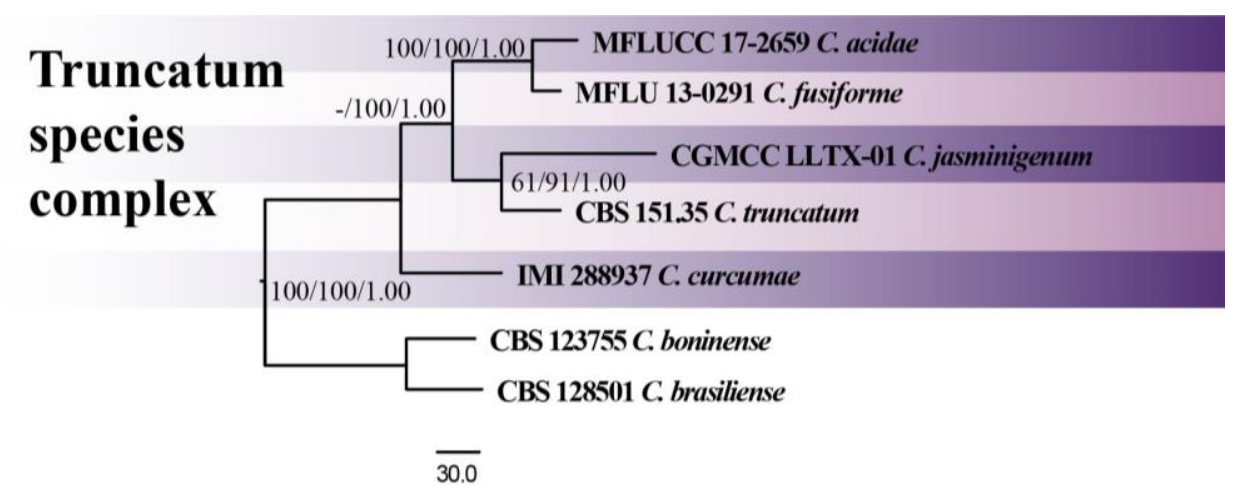

Figure 18 - The truncatum complex. One of the 100 most parsimonious trees obtained from a heuristic search of combined ITS, gapdh, chs-1, act and tub2 sequence data. The MP and ML bootstrap support values $\geq 50 \%$ and BYPP $\geq 0.90$ are indicated at the nodes and branches. The tree is rooted with C. boninense (CBS 123755) and C. brasiliense (CBS 128501). 
Table 17 GenBank accession numbers of the species of the truncatum species complex

\begin{tabular}{|c|c|c|c|c|c|c|c|}
\hline Species name & Type/Reference strain & ITS & gapdh & chs -1 & act & $t u b 2$ & References \\
\hline C. acidae & MFLUCC 17-2659 & MG996505 & MH003691 & MH003694 & MH003697 & MH003700 & Samarakoon et al. (2018) \\
\hline C. curcumae & IMI 288937 & GU227893 & GU228285 & GU228383 & GU227991 & GU228187 & Damm et al. (2009) \\
\hline C. fusiforme & MFLU 13-0291 & KT290266 & KT290255 & KT290253 & KT290251 & KT290256 & Ariyawansa et al. (2015) \\
\hline C. jasminigenum & MFLUCC 10-0273 & HM131513 & & & - & HM153770 & Wikee et al. (2011) \\
\hline C. truncatum & CBS 151.35 & GU227862 & GU228254 & GU228352 & GU227960 & GU228156 & Damm et al. (2009) \\
\hline
\end{tabular}

Colletotrichum truncatum (Schwein.) Andrus \& W.D. Moore, Phytopathology 25: 121 (1935)

Sexual morph not reported. See Damm et al. (2009) for illustrations and descriptions of asexual morph.

Epitype - USA, Pennsylvania, Bethlehem, on pods of Phaseolus lunatus, C.F. Andrus, epitype CBS H-20368 (dried culture), culture ex-epitype CBS 151.35).

Hosts - Arachis hypogaea, Basella sp., Bougainvillea sp., Brassica sp., Capsicum sp., Clitoria ternatea, Corchorus capsularis, Crotalaria sp., Cyperus rotundus, Glycine max, Homo sapiens (corneal ulcer), Limonium sp., Medicago sativa, Opuntia sp., Peperomia magnoliifolia, Phaseolus sp., Stylosanthes hamata, Vigna sp. (Damm et al. 2009), Xanthium occidentale (Shivas et al. 2016), Allium sp. (Matos et al. 2017, Salunkhe et al. 2018a), Fragaria $\times$ ananassa (Bi et al. 2017), Begonia sp. (Zhai et al. 2018), Vitis sp. (Jayawardena et al. 2018, Zhang et al. 2018), Dracaena braunii (Liu et al. 2019), Euphorbia hirta (de Souza et al. 2019), Abutilon theophrasti (Cong et al. 2020), Arachis hypogaea (Yu et al. 2020)

Distribution - Australia, Bangladesh, Brazil, Burkina Faso, Denmark, Gambia, India, Indonesia, Israel, Laos, Mexico, Nepal, Netherlands, Pakistan, Sudan, Tanzania, Trinidad and Tobago, USA, Zimbabwe (Damm et al. 2009), China (Jayawardena et al. 2018, Zhang et al. 2018, Cong et al. 2020)

Notes - Colletotrichum truncatum has a wide distribution (Damm et al. 2009). This is an important pathogen as it causes anthracnose on many economically important plants (Damm et al. 2009, Jayawardena et al. 2016a). Pathogenicity studies for this species are available. Colletotrichum truncatum is also reported to cause keratitis and corneal infections of humans (Hung et al. 2020).

\section{Singleton species}

There are 13 singleton species in Colletotrichum. Most of these species appear to be host specific and restricted to a geographical area. Details of these singletons are provided below. Sequence data of ITS, gapdh, chs-1, act and tub2 sequence data is given in Table 18.

Colletotrichum chlorophyti S. Chandra \& Tandon [as 'chlorophytumi'], Curr. Sci. 34: 565 (1965)

Sexual morph not reported. See Damm et al. (2009) for illustrations and descriptions of asexual morph.

Type - India, Allahabad, Alfred Park, on leaves of Chlorophytum sp., Oct. 1963, S. Chandra holotype IMI 103806, culture ex-type IMI 103806

Hosts/substrates - Chlorophytum sp., Stylosanthes hamata (Damm et al. 2009), Glycine max (Yang et al. 2012b), Moringa oleifera (Cai et al. 2016), Atractylodes chinensis (Sun et al. 2019)

Distribution - Australia, India (Damm et al. 2009), USA (Yang et al. 2012b), China (Cai et al. 2016, Sun et al. 2019) 
Notes - Colletotrichum chlorophyti was described as a leaf spot pathogen of Chlorophytum sp. from India (Damm et al. 2009). This species can be characterized by dark brown chlamydospores in chains and clades (Damm et al. 2009). Whole-genome sequence data (Gan et al. 2017) and pathogenicity studies are available for this species (Cai et al. 2016, Sun et al. 2019).

Colletotrichum citrus-medicae Qian Zhang, Yong Wang bis, Jayawardena \& K.D. Hyde, in Hyde et al., Fungal Diversity: 10.1007/s13225-020-00458-2, [23] (2020)

Sexual morph not reported. See Hyde et al. (2020) for illustrations and descriptions of asexual morph.

Type - China, Kunming Botanical Gardens, Kunming, Yunnan Province, on diseased leaves of Citrus medica, 15 January 2018, Q. Zhang, holotype HGUP 1554, culture ex-type GUCC 1554.

Hosts - Citrus medica (Hyde et al. 2020)

Distribution - China (Hyde et al. 2020)

Notes - Colletotrichum citrus-medicae is closely related to C. sydowii, but differs from its relatively shorter conidia (Hyde et al. 2020). This was isolated from diseased leaves, but pathogenicity studies are needed to confirm this.

Colletotrichum coccodes (Wallr.) S. Hughes, Can. J. Bot. 36: 754 (1958)

Sexual morph not reported. See Liu et al. (2011) for illustrations and descriptions of asexual morph.

Neotype - The Netherlands, Groningen, from Solanum tuberosum tuber, G. Jager, July 1975, dried culture CBS H-10573, culture ex-neotype CBS 369.75.

Hosts/substrates - Rubus coreanus (Kim et al. 2012), Anthurium sp., Beta vulgaris, Capsicum sp., Cucurbita pepo, Globodera rostochiensis, Heterodera pallida, Soil, Solanum sp. (Liu et al. 2011, 2013), Fragaria x ananassa (fruit rot), Iris sp. (Shivas et al. 2016), Viola tricolor (Karamnejadi et al. 2017), Spinacia oleracea (Liu et al. 2020)

Distribution - Korea (Kim et al. 2012), Bulgaria, Czech Republic, Germany, Ireland, Netherlands, South Africa, Switzerland, UK, Yugoslavia (Liu et al. 2011, 2013), Australia (Shivas et al. 2016), Iran (Karamnejadi et al. 2017), USA (Fealko et al. 2019, Liu et al. 2020)

Notes - Colletotrichum coccodes is similar to C. gloeosporioides (Liu et al. 2011). However, in the multilocus phylogeny $C$. coccodes does not group within the $C$. gloeosporioides complex. This species was first isolated from potato black dot disease. There are many host records for this species in Farr \& Rossman (2021), however, most of them are based on morphology alone. Further collections with molecular data are needed to prove the host distribution of this species. Pathogenicity studies for this species are available.

Colletotrichum hsienjenchang I. Hino \& Hidaka, Bull. Miyazaki Coll. Agric. Forest. 6: 93-99 (1934)

Sexual morph not reported. See Sato et al. (2012) for illustrations and descriptions of asexual morph.

Type - Japan, Kanagawa Prefecture, Odawara, diseased culm of Phyllostachys bambusoides collected 2011, holotype IMI 103806, culture ex-type IMI 103806.

Hosts/substrates - Phyllostachys sp. (Sato et al. 2012)

Distribution - Japan (Sato et al. 2012)

Notes - Colletotrichum hsienjenchang was considered as a rare species by Sato et al. (2012). This species appears to be host-specific and restricted to Japan. There are no pathogenicity studies available for this species, even though it was recorded to cause disease.

Colletotrichum metake Sacc., Annls mycol. 6(6): 557 (1908)

Sexual morph not reported. See Sato et al. (2012) for illustrations and descriptions of asexual morph. 
Reference specimen - Japan, Ibaraki Prefecture, Tsukuba, Unknown collector, 2009, culture MAFF241876.

Hosts/substrates - Pleioblastus simoni (Sato et al. 2012)

Distribution - Japan (Sato et al. 2012)

Notes - Colletotrichum metake was described in Italy on dead culms of Arundinaria japonica (Saccardo 1908). This species was found on another bamboo in Japan (Sato et al. 2012). This species has falcate conidia. It is a singleton species, closely related to $C$. hsienjenchang (Jayawardena et al. 2016a). Sato et al. (2012) mentioned it as a rare species. Sequence data are available only for the Japanese culture, hence considered as the reference strain for this species.

Colletotrichum nigrum Ellis \& Halst., in Halsted, New Jersey Agric. Coll. Exp. Sta. Bull.: 297 (1895)

Sexual morph not reported. See Liu et al. (2013) for illustrations and descriptions of asexual morph.

Epitype - Argentina, on Capsicum sp., unknown collection date and collector epitype CBS H-21032, ex-epitype culture CBS 169.49.

Hosts/substrates - Capsicum sp., Cichorium intybus, Fragaria sp. Helianthus tuberosus, Lens culinaris, Solanum lycopersicum (Liu et al. 2013a), Salvia gregii (Guarnaccia et al. 2019), Chenopodium quinoa (Pal \& Testen 2020)

Distribution - Argentina, Canada, Chile, New Zealand, Serbia and Montenegro, UK, USA (Liu et al. 2013a, Pal \& Testen 2020), Italy (Guarnaccia et al. 2019)

Notes - Colletotrichum nigrum is a singleton species with a close affinity to $C$. coccodes and can be identified using ITS sequence data. Colletotrichum nigrum forms significantly longer conidia than $C$. coccodes with a larger L/W ratio (see Liu et al. 2013a). Pathogenicity studies and whole-genome sequence data for this species are available. There are many host records associated with this species. However, most of those studies lack molecular data to confirm the species (Farr \& Rossman 2021).

Colletotrichum orchidophilum Damm, P.F. Cannon \& Crous, in Damm, Cannon, Woudenberg, \& Crous, Stud. Mycol. 73: 83 (2012)

Sexual morph not reported. See Damm et al. (2012b) for illustrations and descriptions of asexual morph.

Type - USA, Hawaii, Oahu, Manoa, from Dendrobium sp., unknown collection date and collector, holotype CBS H-20718, culture ex-type CBS 632.80

Hosts/substrates - Ascocenda sp., Cycnoches aureum, Dendrobium sp., Phalaenopsis sp. (Damm et al. 2012b), Vanilla planifolia (Charron et al. 2018)

Distribution - Panama, UK, USA (Damm et al. 2012b), France (Charron et al. 2018)

Notes - Colletotrichum orchidophilum is basal to the acutatum species complex. This species can be easily recognised by its very narrow, cylindrical conidia, abundantly formed setae and dark brown, uniformly shaped, pyriform to spathulate appressoria (Damm et al. 2012b). Colletotrichum orchidophilum appears to be specific to Orchidaceae. Whole-genome sequence data for this species is available (Baroncelli et al. 2018).

Colletotrichum phaseolorum S. Takim., Ann. phytopath. Soc. Japan 5: 21 (1934)

Sexual morph not reported. See Damm et al. (2009) for illustrations and descriptions of asexual morph.

Type - not designated

Hosts/substrates - Phaseolus sp., Vigna sinensis Damm et al. (2009)

Distribution - Japan (Damm et al. 2009)

Notes - Due to the availability of two strains without a designated type, Jayawardena et al. (2016a) considered this species as doubtful. This species appears to be restricted to Japan. More 
collections are needed to confirm this and epitypification of this species is highly recommended as it is an important pathogen.

Colletotrichum pseudoacutatum Damm, P.F. Cannon \& Crous, in Damm, Cannon, Woudenberg, \& Crous, Stud. Mycol. 73: 91 (2012)

Sexual morph not reported. See Damm et al. (2012b) for illustrations and descriptions of asexual morph.

Type - Chile, Valdivia, San Patricio forest nursery of the Corporación Nacional Forestal near San José de la Mariquina, from seedlings of Pinus radiata, between Dec 1976 and Feb 1977, unknown collector, holotype CBS H-20729, culture ex-holotype CBS 436.77.

Hosts/substrates - Pinus radiata, Syzygium jambos (Damm et al. 2012b, Soares et al. 2017)

Distribution - Brazil (Soares et al. 2017), Chile (Damm et al. 2012b)

Notes - Colletotrichum pseudoacutatum is basal to the acutatum species complex and forms a sister group to a clade containing the acutatum complex and C. orchidophilum. This species can be distinguished from species in the acutatum species complex with gapdh sequence data and by the formation of pale brown, verruculose, irregular-shaped appressoria, and also by the more abundant formation of setae (Damm et al. 2012b).

Colletotrichum pyrifoliae M. Fu \& G.P. Wang, in Fu et al., Persoonia 42: 25 (2018)

See Fu et al. (2019) for illustrations and descriptions of asexual and sexual morph.

Type - China, Hubei Province, Wuhan City, on leaves of P. pyrifolia cv. 'Jinshui', 1 Aug. 2016, M. Fu, holotype HMAS 247825, culture ex-type CGMCC 3.18902.

Hosts/substrates - Pyrus pyrifolia (Fu et al. 2019)

Distribution - China (Fu et al. 2019)

Notes - This species is a confirmed pathogen of Pyrus pyrifolia in China ( $\mathrm{Fu}$ et al. 2019). However, this species seems to be host specific and restricted to China.

Colletotrichum rusci Damm, P.F. Cannon \& Crous, in Damm et al., Fungal Diversity 39: 72 (2009)

Sexual morph not reported. See Damm et al. (2009) for illustrations and descriptions of asexual morph.

Type - ITALY, intercepted JFKIA 151256, on stem of Ruscus, collected 26 Jul. 2002 by A. Towson, holotype CBS H-20365, culture ex-type CBS 119206.

Hosts/substrates - Ruscus sp. (Damm et al. 2009)

Distribution - Italy (Damm et al. 2009)

Notes - Colletotrichum rusci has curved conidia (Damm et al. 2009). Phylogenetically this species is closely related to C. trichellum (Fig. 5). Colletotrichum rusci appears to be host specific and restricted to Italy.

Colletotrichum sydowii Damm, in Marin-Felix et al., Stud. Mycol. 86: 152 (2017)

Sexual morph not reported. See Marlin-Felix et al. (2017) for illustrations and descriptions of asexual morph.

Type - Taiwan, from leaves of Sambucus sp., 18 Dec. 2011, P.W. Crous, holotype CBS H21509, culture ex-type CBS 13581.

Hosts/substrates - Sambucus sp. (Marlin-Felix et al. 2017)

Distribution - Taiwan, China (Marlin-Felix et al. 2017)

Notes - Conidia of this species resemble species of acutatum, boninense and gloeosporioides species complexes. This species can be distinguished by its unique gapdh sequence data (MarlinFelix et al. 2017). Colletotrichum sydowii appears to be host specific and restricted to Taiwan. However, more collections are needed to confirm this.

Colletotrichum trichellum (Fr.) Duke, Trans. Br. mycol. Soc. 13(3-4): 173 (1928) 
Sexual morph not reported. See Damm et al. (2009) for illustrations and descriptions of asexual morph.

Epitype - UK, Berkshire, Binfield, on leaf of Hedera helix, B.C. Sutton, Mar 1964, epitype (dried culture) CBS 217.64.

Hosts/substrates - Hedera sp. (Crouch et al. 2009a, Damm et al. 2009)

Distribution - Canada, Germany, Guatemala, Netherlands, New Zealand, UK (Damm et al. 2009), USA (Crouch et al. 2009a)

Notes - Colletotrichum trichellum has curved conidia and is closely related to C. rusci (Damm et al. 2009). This species is recorded to cause leaf spot of Hedera sp. (Hyde et al. 2009b). However, pathogenicity data for this species is lacking.

Table 18 GenBank accession numbers of the singleton species

\begin{tabular}{|c|c|c|c|c|c|c|c|}
\hline Species name & Type/Reference strain & ITS & gapdh & chs-1 & act & tub2 & References \\
\hline C. citrus-medicae & GUCC 1554 & MN959910 & MT006331 & MT006328 & MT006325 & & Hyde et al. (2020) \\
\hline C. chlorophyti & IMI 103806 & GU227894 & GU228286 & GU228384 & GU227992 & GU228188 & Damm et al. (2009) \\
\hline C. coccodes & CBS 369.75 & HM171679 & HM171673 & JQ005796 & HM171667 & JQ005859 & Liu et al. (2011) \\
\hline C. hsienjenchang & MAFF 243051 & AB738855 & & AB738846 & AB738845 & & Sato et al. (2012) \\
\hline C. metake & MAFF 244029 & AB738859 & & & & & Sato et al. (2012) \\
\hline C. nigrum & CBS 169.49 & JX546838 & JX546742 & JX546693 & JX546646 & JX546885 & Liu et al. (2013) \\
\hline C. orchidophilum & CBS 632.80 & JQ005778 & & JQ005799 & JQ005841 & JQ005862 & Damm et al. (2012b) \\
\hline C. phaseolorum & CBS 158.36 & GU227897 & GU228289 & GU228387 & GU227995 & GU228191 & Damm et al. (2009) \\
\hline C. pseudoacutatum & CBS 436.77 & JQ948480 & JQ948811 & JQ949141 & JQ949801 & JQ950131 & Damm et al. (2012b) \\
\hline C. pyrifolia & CGMCC 3.18902 & MG748078 & MG747996 & MG747914 & MG747768 & MG748158 & Fu et al. (2019) \\
\hline C. rusci & CBS 119206 & GU227818 & GU228210 & GU228308 & GU227916 & GU228112 & Damm et al. (2009) \\
\hline C. sydowii & CBS135819 & KY263783 & KY263785 & KY263787 & KY263791 & KY263793 & Marlin-Felix et al. (2017) \\
\hline C. trichellum & CBS 217.64 & GU227812 & GU228204 & GU228302 & GU227910 & GU228106 & Damm et al. (2009) \\
\hline
\end{tabular}

Table 19 Morphospecies of Colletotrichum

\begin{tabular}{|c|c|c|c|c|}
\hline Species & Typification Details & Host-Substratum & Country & Reference \\
\hline $\begin{array}{l}\text { Colletotrichum acaciae } \\
\text { Gutner }\end{array}$ & N/A & On Acacia harpophylla in green house & Russia & $\begin{array}{l}\text { Bondartseva-Monteverde et al. } \\
\text { (1936) }\end{array}$ \\
\hline $\begin{array}{l}\text { C. acalyphae G. W. } \\
\text { Wilson }\end{array}$ & N/A & On leaves of Acalypha ciliata & India & Wilson (1960) \\
\hline $\begin{array}{l}\text { C. acanthostachydis } \\
\text { Novoss. }\end{array}$ & N/A & On seeds of Acanthostachys strobilaceae & Sweden & Novosselova (1938) \\
\hline C. acanthosyridis Speg. & Spegazzini, Jan. 1906 & On leaves of Acanthosyridis falcatae & Argentina & Spegazzini (1911) \\
\hline C. achilleae Dobrozr. & N/A & On leaves of Achillea millefolii & Russia & Dobrozrakova (1927) \\
\hline $\begin{array}{l}\text { C. achyrantheum (Tassi) } \\
\text { Petr. \& Syd. }\end{array}$ & N/A & On dried stems of Achryanthis verschaffeltii & Italy & Petrak \& Sydow (1927) \\
\hline $\begin{array}{l}\text { C. actinidiae Togashi \& } \\
\text { Onuma }\end{array}$ & N/A & On living leaves of Actinidia callosa var. arguta & Japan & Togashi \& Unuma (1934) \\
\hline
\end{tabular}


Table 19 Continued.

\begin{tabular}{|c|c|c|c|c|}
\hline Species & Typification Details & Host-Substratum & Country & Reference \\
\hline $\begin{array}{l}\text { C. adustum (Ellis \& G. } \\
\text { Martin) Ellis \& Underw. }\end{array}$ & Holotype NY, Martin, Mar. 1886 & On leaves of Citrus & US & Underwood (1891) \\
\hline C. aecidiicola Negru & N/A & $\begin{array}{l}\text { Around aecidia on leaves of Berberis vulgaris and } B \text {. } \\
\text { dielsiana }\end{array}$ & Romania & Negru (1958) \\
\hline C. aeciicola Tehon & Holotype ILL 22416 & On aecidium of Puccinia asterum & USA & Tehon (1933) \\
\hline $\begin{array}{l}\text { C. aesculi E. Rădul. \& } \\
\text { Negru }\end{array}$ & N/A & On leaves of Aesculus hippocastanum & Romania & Radulescu \& Negru (1963) \\
\hline C. agatinum (Sacc.) Petr. & N/A & On stems of Agati grandiflorae & Philipines & Petrak (1929) \\
\hline C. ailanthi Tognini & N/A & On petioles of Ailanthus glandulosa & Italy & Tognini (1895) \\
\hline C. ajugae Siemaszko & N/A & On living leaves of Ajuga repens & $\begin{array}{l}\text { Georgia, Armenia } \\
\text { \& Azerbaijan }\end{array}$ & Siemaszko (1923) \\
\hline C. aletridis Henn. & Yoshinaga 27 & On leaves of Aletris japonica & Japan & Hennings (1904a) \\
\hline C. alibertiae Novoss. & N/A & On Alibertia celtis, in greenhouses & Russia & $\begin{array}{l}\text { Bondartseva-Monteverde et al. } \\
\text { (1936) }\end{array}$ \\
\hline C. allii Av.-Saccá & N/A & N/A & N/A & Averna-Saccá (1917) \\
\hline C. alni Siemaszko & N/A & On living leaves of Alnus glutinosa & $\begin{array}{l}\text { Georgia, Armenia } \\
\text { \& Azerbaijan }\end{array}$ & Siemaszko (1923) \\
\hline C. aloes Bacc. & Baldrati 21 & On leaves of Aloe abyssinica & Eritrea & Baccarini (1917) \\
\hline C. alstoniae (Sacc.) Petr. & Baker 3739 & On dead leaves of Alstonia scholaris & Philippines & Petrak (1929) \\
\hline C. althaeae Southw. & N/A & $\begin{array}{l}\text { Pathogenic on leaves, petioles and stalks of Alcea } \\
\text { rosea }\end{array}$ & US & Southworth (1980) \\
\hline C. ampelinum Cavara & N/A & On branches of Vitis sp. & Italy & Cavara (1889a) \\
\hline C. ampelopsidis Sawada & N/A & On Ampelopsis heterophylla var. ciliate & Taiwan & Sawada (1944) \\
\hline C. anacardii Kelkar & Holotype MACS 102 & On leaves of Rhus mysorensis & India & Kelkar \& Rao (1962) \\
\hline C. andropogonis Zimm. & N/A & On leaves of Andropogonis sorghi and Sorghi vulgaris & Africa & Zimmermann (1904) \\
\hline C. annonicola Speg. & Spegazzini, Apr. 1906 & On living leaves of Annona cherimolia & Argentina & Spegazzini (1911) \\
\hline C. antarcticum Henn. & Werth, 26 Dec. 1901 & On leaves of Poea cookie & $\begin{array}{l}\text { Crozet Island, } \\
\text { Antarctica }\end{array}$ & Hennings (1906) \\
\hline C. anthurii Delacr. & N/A & On leaves of Anthurii & Germany & Neergaard (1943) \\
\hline C. antirrhini F.C. Stewart & N/A & N/A & N/A & Stewart (1900) \\
\hline C. aquatile R. Sprague & N/A & On leaves of Catabrosa aquatic & US & Sprague (1957) \\
\hline C. arachidis Sawada & N/A & On leaves of Arachis hypogaea & Taiwan & Sawada (1959) \\
\hline $\begin{array}{l}\text { C. araliae Kamal \& R.P. } \\
\text { Singh }\end{array}$ & N/A & On leaves of Aralia nobilis & India & Kamal \& Singh (1980) \\
\hline C. ardisiae C.C. Chen & N/A & On leaves of Ardisia crispa var. dielsii & Taiwan & Chen (1968a) \\
\hline C. arecae Syd. \& P. Syd. & Merrill 8958, Merrill 8919 & On dead leaf sheaths of Areca catechu & Philippines & Sydow \& Sydow (1914a) \\
\hline C. ari (Pass.) Bat. & N/A & On the drooping leaves of Arum italicum & Italy & Höhnel (1919) \\
\hline
\end{tabular}


Table 19 Continued.

\begin{tabular}{|c|c|c|c|c|}
\hline Species & Typification Details & Host-Substratum & Country & Reference \\
\hline $\begin{array}{l}\text { C. arisaematis H.C. } \\
\text { Greene }\end{array}$ & Greene, 14 Jun. 1954 & On leaves of Arisaema atrorubens & US & Greene (1955) \\
\hline C. arjunae V.P. Sahni & Holotype IMI 108642 & On leaves of Terminalia arjuna & India & Sahni (1968) \\
\hline $\begin{array}{l}\text { C. armeriae Nicolas \& } \\
\text { N. Aggéry }\end{array}$ & N/A & On dead stems and leaves of Armeria plantaginea & France & Nicolas \& Aggéry (1942) \\
\hline C. artabotrydis Sawada & N/A & On Artabotrys uncinatus & Taiwan & Sawada (1943) \\
\hline $\begin{array}{l}\text { C. arthraxonis (S. } \\
\text { Ahmad) S. Ahmad }\end{array}$ & N/A & On leaves of Arthraxon serrulatus & Pakistan & Ahmad (1956) \\
\hline C. artocarpi Delacr. & N/A & On leaves of Artocarpi incisae & France & Delacroix (1905) \\
\hline $\begin{array}{l}\text { C. asiaticum U.P. Singh } \\
\text { \& H.P. Upadhyay }\end{array}$ & N/A & On leaves of Crinum asiaticum & India & Pavgi \& Upadhyay (1964) \\
\hline $\begin{array}{l}\text { C. aspleniifoliae Pavgi } \\
\text { \& U.P. Singh }\end{array}$ & N/A & On leaves of Launaea aspleniifolia & India & Pavgi \& Singh (1965) \\
\hline C. atractyli Koval & N/A & On leaves of Atractylodes ovata & $\begin{array}{l}\text { Maritime Territory, } \\
\text { Russian }\end{array}$ & Koval (1961) \\
\hline $\begin{array}{l}\text { C. atramentarium (Berk. } \\
\& \text { Broome) Taubenh. }\end{array}$ & N/A & On decaying stems of Solanum tuberosum & UK & Taubenhaus (1916) \\
\hline C. atriplicinum Syd. & Bürgener, 12 Dec. 1929 & On dead stems of Atriplex patula & Germany & Sydow (1930) \\
\hline C. atropae Klaptzova & N/A & On leaves of Atropa belladonna & $\begin{array}{l}\text { 'Western former } \\
\text { Soviet Union' }\end{array}$ & Klaptzova (1940) \\
\hline C. аисиbae Gutner & N/A & On dead leaves Aucuba japonica & Russia & Gutner (1933a) \\
\hline $\begin{array}{l}\text { C. aucubanum (Sacc.) } \\
\text { Petr. \& Syd. }\end{array}$ & Borg 508 & On dead leaves of Аисиba japonica & Malta & Petrak \& Sydow (1927) \\
\hline C. aureum Corda & N/A & On Spliaeriis and Scleromycetibus & Germany & Corda (1837) \\
\hline C. averrhoae Bat. & N/A & On Averrhoa carambola & Brazil & Batista (1952) \\
\hline $\begin{array}{l}\text { C. azaleae Ellis \& } \\
\text { Everh. }\end{array}$ & Holotype NY, Nash 1991 & On leaves of Azalea viscose & US & Ellis \& Everhart (1895) \\
\hline $\begin{array}{l}\text { C. bakeri (Syd. \& P. } \\
\text { Syd.) Mundk. }\end{array}$ & Baker 3871 & On dead stems and petioles of Ricinus communis & Philippines & Mundkur (1938) \\
\hline C. bauhiniae Gutner & N/A & On Bauhinia rosea, in greenhouses & Russia & $\begin{array}{l}\text { Bondartseva-Monteverde et al. } \\
\text { (1936) }\end{array}$ \\
\hline C. begoniae Bat. & N/A & On Begonia gracilis & Brazil & Batista (1952) \\
\hline $\begin{array}{l}\text { C. bhandardarense } \\
\text { Patw. }\end{array}$ & N/A & On leaves of Arisaema murrayi & India & Patwardhan (1966) \\
\hline $\begin{array}{l}\text { C. bignoniae-igneae } \\
\text { Rangel }\end{array}$ & N/A & On leaves of Bignoniaw igneae & Brazil & Maublanc \& Rangel (1915) \\
\hline
\end{tabular}


Table 19 Continued.

\begin{tabular}{|c|c|c|c|c|}
\hline Species & Typification Details & Host-Substratum & Country & Reference \\
\hline C. biologicum Chaudhuri & N/A & On withering stalk of Solanum tuberosum & Germany & Chaudhuri (1924) \\
\hline C. biscutellae Moesz & N/A & On dried petioles of Biscutella alpestris & former Yugoslavia & Degen (1938) \\
\hline C. bixae Sawada & N/A & On leaves of Bixa orellana & Taiwan & Sawada (1959) \\
\hline $\begin{array}{l}\text { C. blighiae Gonz. Frag. \& } \\
\text { Cif. }\end{array}$ & Ciferri, Jan. 1927 & On leaves of Sapindaceae & $\begin{array}{l}\text { Dominican } \\
\text { Republic }\end{array}$ & González \& Ciferri (1927) \\
\hline C. boehmeriae Sawada & N/A & On living leaves and stalks of Boehmeria nivea & Taiwan & Saccardo (1922) \\
\hline $\begin{array}{l}\text { C. boerhaviae Pavgi \& } \\
\text { U.P. Singh }\end{array}$ & N/A & On leaves of Boerhavia diffusa & India & Pavgi \& Singh (1965) \\
\hline $\begin{array}{l}\text { C. bougainvilleae } \\
\text { Novoss. }\end{array}$ & N/A & On Bougainvillea glabra, in green houses & Russia & $\begin{array}{l}\text { Bondartseva-Monteverde et al. } \\
\text { (1936) }\end{array}$ \\
\hline C. brachytrichum Delacr. & N/A & On leaves of Theobromae cacao & Trinidad & Delacroix (1905) \\
\hline $\begin{array}{l}\text { C. brassicae Schulzer \& } \\
\text { Sacc. }\end{array}$ & N/A & $\begin{array}{l}\text { On rotting stems of Brassicae oleraceae var. } \\
\text { caulocarpae }\end{array}$ & Croatia & Schulzer \& Saccardo (1884) \\
\hline C. briosii Turconi & N/A & On leaves of Cinnamomi burmanni & Italy & Saccardo (1908) \\
\hline $\begin{array}{l}\text { C. bryoniae (Ferraris) } \\
\text { Maire }\end{array}$ & N/A & On leaves of Bryoniae dioicae & Italy & Maire (1917) \\
\hline C. burserae Novoss. & N/A & On Bursera brasiliensis, in greenhouses & Russia & $\begin{array}{l}\text { Bondartseva-Monteverde et al. } \\
\text { (1936) }\end{array}$ \\
\hline C. cajani Rangel & N/A & On leaves of Cajani indici & Brazil & Rangel (1915) \\
\hline $\begin{array}{l}\text { C. calotropidis Rao \& } \\
\text { M.A. Salam }\end{array}$ & N/A & On leaves of Calotropis gigantea & India & Rao \& Salam (1960) \\
\hline C. canangae Koord. & N/A & On the leaves of Cananga odorata & Java & Koorders (1907) \\
\hline $\begin{array}{l}\text { C. canavaliae Gonz. Frag. } \\
\& \text { Cif. }\end{array}$ & Ciferri, Aug. 1925 & On dry stems of Canavalia maritima & $\begin{array}{l}\text { Dominican } \\
\text { Republic }\end{array}$ & Petrak's Lists volume 3 \\
\hline C. canavaliicola Sawada & N/A & On pods of Canavalia ensiformis & Taiwan & Sawada (1959) \\
\hline C. capparis Hasija & $\mathrm{N} / \mathrm{A}$ & On leaves of Capparis sepiaria & India & Hasija (1962b) \\
\hline $\begin{array}{l}\text { C. caricae F. Stevens \& } \\
\text { J.G. Hall }\end{array}$ & N/A & On fruits and leaves of Ficus caricae & US & Stevens \& Hall (1909) \\
\hline $\begin{array}{l}\text { C. carpophilum Kellerm. } \\
\text { \& Swingle }\end{array}$ & $\begin{array}{l}\text { Kellerman \& Swingle, May } \\
1888, \text { Kellerman \& Swingle, Jun. } \\
1888\end{array}$ & On living fruit of Astragalus caryocarpus & US & Kellerman \& Swingle (1888) \\
\hline C. carveri Ellis \& Everh. & Holotype NY, Carver s.n. & On leaves of Thea sinensis & US & Ellis \& Everhart (1902) \\
\hline C. catechu Died. & Sen, 19 Dec. 1907 (Butler 1660) & On leaves of Areca catechu & Bangladesh & Sydow et al. (1916) \\
\hline C. catenulatum F. Stevens & Holotype ILL, Stevens 32 & On living leaves of Agave angustifolia var. marginata & Guyana & Stevens (1930) \\
\hline $\begin{array}{l}\text { C. caulicola Heald \& } \\
\text { F.A. Wolf }\end{array}$ & $\begin{array}{l}\text { Holotype BPI, Heald \& Wolf, } \\
1963\end{array}$ & On living stems of Phaseolus vulgaris & US & Heald \& Wolf (1911) \\
\hline C. cavendishii Petr. & $\begin{array}{l}\text { Schönau, } 26 \text { Jun. 1918, Schönau, } \\
31 \text { Jul. } 1919\end{array}$ & On living leaves of Musa cavendishii & Germany & Petrak (1925) \\
\hline
\end{tabular}


Table 19 Continued.

\begin{tabular}{|c|c|c|c|c|}
\hline Species & Typification Details & Host-Substratum & Country & Reference \\
\hline C. cecropiae Viégas & N/A & On Cecropia & Brazil & Viégas (1946) \\
\hline $\begin{array}{l}\text { C. celastri-paniculatae } \\
\text { Thite \& M.S. Patil }\end{array}$ & N/A & On leaves of Celastrus paniculatus & India & Thite \& Patil (1975) \\
\hline C. cerei Earle & Tracey 7309 & On dead leaves of Cereus triangularis & US & Earle (1902) \\
\hline $\begin{array}{l}\text { C. chaetomium (Wallr.) } \\
\text { S. Hughes }\end{array}$ & N/A & On Cucubitaceae & US & Hughes (1958) \\
\hline $\begin{array}{l}\text { C. chaetostromum } \\
\text { (Berk. \& Broome) } \\
\text { Duke }\end{array}$ & N/A & On dead seed of Fraxinus excelsior & UK & Duke (1928) \\
\hline $\begin{array}{l}\text { C. chamaeropis Ferraris } \\
\text { \& Gabotto }\end{array}$ & N/A & On leaves of Chamaeropis excelsae & Italy & Gabotti (1914) \\
\hline C. chardonianum Nolla & N/A & On living leaves of Allium сера & Puerto Rico & Nolla (1926) \\
\hline $\begin{array}{l}\text { C. ciliatum T.S. } \\
\text { Ramakr. \& K. Ramakr. }\end{array}$ & N/A & On Cymbopogon polyneuros & India & $\begin{array}{l}\text { Ramakrishnan \& Ramakrishnan } \\
\text { (1947) }\end{array}$ \\
\hline C. cinchonae Koord. & N/A & On leaves of Cinchonae sp. & Java & Koorders (1907) \\
\hline $\begin{array}{l}\text { C. cinctum (Berk. \& } \\
\text { M.A. Curtis) Stoneman }\end{array}$ & Ravenel 5437 & On Orchidaceae & US & Stoneman (1898) \\
\hline C. cinnamomi Tharp & $\begin{array}{l}\text { Holotype BPI, Tharp, } 13 \text { Sept. } \\
1915\end{array}$ & On living leaves of Cinnamomum zeylanicum & US & Tharp (1917) \\
\hline C. cirrhopetali Sawada & $\mathrm{N} / \mathrm{A}$ & On leaves of Cirrhopetalum uraiense & Taiwan & Sawada (1959) \\
\hline C. clavijae Gutner & N/A & On Clavija riedeliana and $C$. spinosa, in greenhouses & Russia & $\begin{array}{l}\text { Bondartseva-Monteverde et al. } \\
\text { (1936) }\end{array}$ \\
\hline C. clerodendri Died. & N/A & On leaves of Clerodendri infortunati & India & Sydow et al. (1916) \\
\hline C. clusiae Petr. & Zerny, 18 Aug. 1927 & On Clusia & Brazil & Petrak (1947b) \\
\hline C. cocae Speg. & N/A & On leaves of Erythroxylum coca & Argentina & Spegazzini (1920) \\
\hline C. coffeanum F. Noack & N/A & On Coffea & Brazil & Noack (1901) \\
\hline C. coffeophilum Speg. & Tonduz, Oct. 1897 & On living leaves of Coffea & Costa Rica & Spegazzini (1918a) \\
\hline $\begin{array}{l}\text { C. commelinae Ellis \& } \\
\text { Everh. }\end{array}$ & Holotype NY, Nash 1798 & On leaves of Commelina angustifolia & US & Ellis \& Everhart (1895) \\
\hline $\begin{array}{l}\text { C. compactum T.S. } \\
\text { Ramakr. }\end{array}$ & N/A & On Albizzia lebbek & India & Ramakrishnan (1951) \\
\hline $\begin{array}{l}\text { C. concentricum } \\
\text { Massee }\end{array}$ & N/A & On fruit of Trichosanthes anguina & UK & Massee (1913) \\
\hline $\begin{array}{l}\text { C. consociatum J.C. } \\
\text { Lindq. }\end{array}$ & N/A & On Solidago chilensis & Argentina & Lindquist (1948) \\
\hline $\begin{array}{l}\text { C. conspicuum Syd. \& } \\
\text { P. Syd. }\end{array}$ & Collado 6229 & On living leaves of Erythropalum scandens & Philippines & Sydow \& Sydow (1920) \\
\hline
\end{tabular}


Table 19 Continued.

\begin{tabular}{|c|c|c|c|c|}
\hline Species & Typification Details & Host-Substratum & Country & Reference \\
\hline $\begin{array}{l}\text { C. corchori Pavgi \& } \\
\text { U.P. Singh }\end{array}$ & N/A & On leaves of Corchorus acutangulus & India & Ikata \& Yoshida (1940) \\
\hline C. cordiae Allesch. & N/A & On Cordia maritima & Germany & Allescher (1902) \\
\hline C. cordylines Pollacci & December 1894 & On leaves of Cordylinae indivisae & Italy & Pollacci (1896) \\
\hline $\begin{array}{l}\text { C. corni (Woron.) } \\
\text { Vassiljevsky }\end{array}$ & N/A & N/A & US & $\begin{array}{l}\text { Vassiljevsky \& Karakulin } \\
\text { (1950) }\end{array}$ \\
\hline $\begin{array}{l}\text { C. corylifoliae Pavgi \& } \\
\text { U.P. Singh }\end{array}$ & N/A & On leaves of Psoralea corylifolia & India & Pavgi \& Upadhyay (1964) \\
\hline $\begin{array}{l}\text { C. corynocarpi Sousa } \\
\text { da Câmara }\end{array}$ & N/A & On dry leaves of Corynocarpus laevigatus & Portugal & Sousa da Câmara (1930) \\
\hline $\begin{array}{l}\text { C. cradwickii C.K. } \\
\text { Bancr. }\end{array}$ & N/A & On shoots of Theobroma cacao & Jamaica & Bancroft (1910) \\
\hline $\begin{array}{l}\text { C. crassipes }(\text { Speg.) } \\
\text { Arx }\end{array}$ & N/A & On berries of Vitis viniferae & Italy & von $\operatorname{Arx}(1957 b)$ \\
\hline $\begin{array}{l}\text { C. crossandrae Patel, } \\
\text { Kamat \& C.B. Pande }\end{array}$ & N/A & On Crossandra infundibuliformis & India & Patel \& Pande (1953) \\
\hline C. crotalariae Petch & Holotype PDA 4946 & On leaves of Crotalaria striata & Sri Lanka & Petch (1917) \\
\hline $\begin{array}{l}\text { C. crotalariae-junceae } \\
\text { Sawada }\end{array}$ & N/A & On stem of Crotalaria juncea & Taiwan & Sawada (1959) \\
\hline $\begin{array}{l}\text { C. crotonicola } \text { Pavgi \& } \\
\text { U.P. Singh }\end{array}$ & N/A & On leaves of Croton sparsiflorus & India & Pavgi \& Singh (1964) \\
\hline C. cryptostegiae Chipl. & Holotype MACS 298 & On leaves of Cryptostegia grandiflora & India & Chiplonkar (1969) \\
\hline $\begin{array}{l}\text { C. curvatum Briant \& } \\
\text { E.B. Martyn }\end{array}$ & N/A & On live stems of Crotalaria juncea & Trinidad-Tobago & Briant \& Martyn (1929) \\
\hline $\begin{array}{l}\text { C. curvisetum } \mathrm{F} \text {. } \\
\text { Stevens }\end{array}$ & $\begin{array}{l}\text { ILL, Stevens 3594, ILL, Stevens } \\
5830\end{array}$ & On leaves of Hura crepitans & Puerto Rico & Stevens (1917) \\
\hline C. cyclanthis Novoss. & N/A & $\begin{array}{l}\text { On Cyclanthus cristatus and on } C \text {. discolor, in } \\
\text { greenhouses }\end{array}$ & Russia & $\begin{array}{l}\text { Bondartseva-Monteverde et al. } \\
\text { (1936) }\end{array}$ \\
\hline $\begin{array}{l}\text { C. cyclobalanopsidis } \\
\text { Sawada }\end{array}$ & N/A & On young leaves of Cyclobalanopsis glauca & Taiwan & Sawada (1959) \\
\hline $\begin{array}{l}\text { C. cylindricum S.N.S. } \\
\text { Srivast. }\end{array}$ & N/A & On Pothos scandens & India & Srivastava (1953) \\
\hline $\begin{array}{l}\text { C. cyperacearum Pavgi } \\
\text { \& U.P. Singh }\end{array}$ & N/A & On leaves of Theropogon pallidus & India & Pavgi \& Singh (1964) \\
\hline $\begin{array}{l}\text { C. dahliae M.E.A. } \\
\text { Costa \& Sousa da } \\
\text { Câmara }\end{array}$ & N/A & On Chrysanthemum and Dahlia & Portugal & $\begin{array}{l}\text { Costa \& Souza da Camara } \\
\text { (1953) }\end{array}$ \\
\hline
\end{tabular}


Table 19 Continued.

\begin{tabular}{|c|c|c|c|c|}
\hline Species & Typification Details & Host-Substratum & Country & Reference \\
\hline C. dalbergiae C.C. Chen & N/A & On leaves of Dalbergia sissoo & Taiwan & Chen $(1968 b)$ \\
\hline $\begin{array}{l}\text { C. damasonii Bond.- } \\
\text { Mont. }\end{array}$ & N/A & On fruits and seeds of Damasonium alismatoides & Sweden & $\begin{array}{l}\text { Bondartzeva-Monteverde } \\
\text { (1938) }\end{array}$ \\
\hline $\begin{array}{l}\text { C. daphnes-ponticae } \\
\text { Siemaszko }\end{array}$ & N/A & On dead leaves of Daphne pontica & $\begin{array}{l}\text { Georgia, Armenia } \\
\text { \& Azerbaijan }\end{array}$ & Siemaszko (1923) \\
\hline C. daphniphylli Dzhalag. & N/A & On leaves of Daphniphyllum macropodium & Azerbaijan & Dzhalagoniya (1962) \\
\hline C. dasturii Roy bis & N/A & On Piper betle & India & Roy (1948) \\
\hline $\begin{array}{l}\text { C. decosteae (Henn.) Petr. } \\
\& \text { Syd. }\end{array}$ & Neger 24 & On dead leaves of Decostea scandens & Chile & Petrak \& Sydow (1927) \\
\hline C. derridis Hoof & N/A & On Derris elliptica & Taiwan & Hoof (1949) \\
\hline $\begin{array}{l}\text { C. dianellae F. Stevens \& } \\
\text { P.A. Young }\end{array}$ & N/A & On leaves of Dianella odorata & Hawaii & Stevens (1925) \\
\hline C. dichaeae Henn. & Behnick, 2 Dec. 1904 & On dead leaves of Dichaea vaginata & Germany & Hennings (1905a) \\
\hline C. dichorisandrae Rangel & N/A & On leaves of Dichorisandrae thyrsiflorae & Brazil & Maublanc \& Rangel (1915) \\
\hline C. dictamni Hollós & N/A & On leaves of Dictamni fraxinella & Hungaria & Hollós (1908) \\
\hline $\begin{array}{l}\text { C. digitalis (Rostr.) } \\
\text { Moesz }\end{array}$ & N/A & On leaves of Digitalis purpurea & Spain & Moesz (1931) \\
\hline C. dioscoreae Av.-Saccá & N/A & On Dioscorea & Brazil & Averna-Saccá (1917) \\
\hline $\begin{array}{l}\text { C. dominicanum Gonz. } \\
\text { Frag. \& Cif. }\end{array}$ & Bosch, Feb.-Jun. 1925 & On dead fruit of Hibiscus brasiliensis & $\begin{array}{l}\text { Dominican } \\
\text { Republic }\end{array}$ & González \& Ciferri (1925) \\
\hline C. dracaenae Allesch. & $\begin{array}{l}\text { Holotype, M-0090064, J.E. } \\
\text { Weiss, ap } 1895\end{array}$ & On faded leaves of Dracaena latifolia & Germany & Allescher (1902) \\
\hline $\begin{array}{l}\text { C. dracaenae-fragrantis } \\
\text { (H. Mori) Petr. \& Syd. }\end{array}$ & N/A & On leaves of Dracaena fragrans & Romania & Petrak \& Sydow (1925) \\
\hline $\begin{array}{l}\text { C. dracaenicola Sacc. \& } \\
\text { Trotter }\end{array}$ & N/A & On leaves of Dracaena frafrantis & Italy & Saccardo \& Trotter (1913) \\
\hline C. durionis Koord. & N/A & On leaves of Durionis zibethini & Java & Koorders (1907) \\
\hline C. echinatum Massee & Holotype K(M), Johnson 131 & On the bark of an unknown tree & Ghana & Massee (1906) \\
\hline $\begin{array}{l}\text { C. effiguratum Syd. \& P. } \\
\text { Syd. }\end{array}$ & N/A & On living leaves of Paphiopedilum roezlii & Germany & Sydow \& Sydow (1900) \\
\hline C. elaeagni Siemaszko & N/A & On living and dead leaves of Elaeagnus & $\begin{array}{l}\text { Georgia, Armenia } \\
\& \text { Azerbaijan }\end{array}$ & Siemaszko (1923) \\
\hline C. elasticae Tassi & N/A & On decaying leaves of Fici elasticae & Italy & Tassi (1900) \\
\hline C. eleagni Siemaszko & N/A & On decaying leaves of Elaeagni sp. & Caucasus & Siemaszko (1923) \\
\hline C. elmeri Syd. & Elmer 15663 & On leaves of Cryptosperma merkusii & Philippines & Sydow (1925a) \\
\hline $\begin{array}{l}\text { C. ensetes (Sacc. \& } \\
\text { Scalia) Petr. \& Syd. }\end{array}$ & N/A & On decaying leaves of Musa ensetes & Portugal & Petrak \& Sydow (1927) \\
\hline C. epiphylli Tassi & N/A & On decaying Epiphylli ackermanni & Italy & Tassi (1904) \\
\hline
\end{tabular}


Table 19 Continued.

\begin{tabular}{|c|c|c|c|c|}
\hline Species & Typification Details & Host-Substratum & Country & Reference \\
\hline C. eranthemi V. Rao & Holotype IMI 103771 & On leaves of Eranthemum bicolor & India & Kelkar \& Rao (1962) \\
\hline C. erumpens (Sacc.) Sacc. & N/A & On stems of Ricinus communis & China & Saccardo (1884) \\
\hline C. eryngii (Desm.) Duke & N/A & On Eryngium & UK & Duke (1928) \\
\hline $\begin{array}{l}\text { C. erythrinae Ellis \& } \\
\text { Everh. }\end{array}$ & NY, Nash 2123 & On leaves of Erythrina herbacea & US & Ellis \& Everhart (1895) \\
\hline C. eucalypti Bitanc. & N/A & N/A & N/A & N/A \\
\hline $\begin{array}{l}\text { C. euchroum Syd. \& P. } \\
\text { Syd. }\end{array}$ & Raimundo s.n. & On leaves of Euphorbia neriifolia & Philippines & Sydow \& Sydow (1913) \\
\hline C. euryae Sawada & N/A & On Eurya japonica & Taiwan & Sawada (1944) \\
\hline $\begin{array}{l}\text { C. eustictum (Sacc.) Petr. } \\
\text { \& Syd. }\end{array}$ & Saccardo, Nov. 1904 & On leaves of Oreodaphne foetens & Italy & Petrak \& Sydow (1924) \\
\hline C. exiguum Penz. \& Sacc. & $\mathrm{N} / \mathrm{A}$ & On leave of Spiraeae arunci & Switzerland & Penzig \& Saccardo (1884) \\
\hline $\begin{array}{l}\text { C. exoticum Pavgi \& U.P. } \\
\text { Singh }\end{array}$ & N/A & On leaves of Murraya exotica & India & Pavgi \& Singh (1964) \\
\hline C. extorre Sacc. & Borg 511 & On leaves of Sciodaphyllum digitatum & Malta & Saccardo (1915) \\
\hline C. fagopyri Sawada & N/A & On stem of Fagopyrum tataricum & Taiwan & Sawada (1959) \\
\hline $\begin{array}{l}\text { C. fici-elasticae Sacc. \& } \\
\text { D. Sacc. }\end{array}$ & N/A & On leaves of Fici elastcae & Italy & Saccardo (1906) \\
\hline C. ficus Koord. & N/A & Pathogenic on leaves of Ficus elastica & Java & Koorders (1905) \\
\hline C. flacourtiae Rangel & $\mathrm{N} / \mathrm{A}$ & On fruit of Flacourtia ramontchi & Brazil & Rangel (1926) \\
\hline $\begin{array}{l}\text { C. fluggeae Thite \& M.S. } \\
\text { Patil }\end{array}$ & N/A & On leaves of Flueggea leucopyrus & India & Thite \& Patil (1975) \\
\hline $\begin{array}{l}\text { C. foliicola (Nishida) } \\
\text { Sawada }\end{array}$ & N/A & On living leaves of Citrus & Japan & Sawada (1959) \\
\hline $\begin{array}{l}\text { C. fructigenum (Berk.) } \\
\text { Vassiljevsky }\end{array}$ & Holotype K(M) 110093 & On fruit of Pyrus communis & UK & $\begin{array}{l}\text { Vassiljevsky \& Karakulin } \\
\text { (1950) }\end{array}$ \\
\hline C. funtumiae Petch & Holotype PDA 4201 & On leaves of Funtumia elastica & Sri Lanka & Petch (1917) \\
\hline $\begin{array}{l}\text { C. fusarioides (Ellis \& } \\
\text { Kellerm.) O’Gara }\end{array}$ & $\begin{array}{l}\text { Holotype NY, Kellerman, Aug. } \\
1884\end{array}$ & On leaves of Asclepias cornuti & US & Dearness (1928) \\
\hline $\begin{array}{l}\text { C. fuscocoriaceum } \\
\text { (Rehm) Petr. }\end{array}$ & Reyes, Sept. 1913 (Baker 1841) & On leaves of Antidesma & Phillippines & Petrak (1934) \\
\hline $\begin{array}{l}\text { C. gangeticum Pavgi \& } \\
\text { U.P. Singh }\end{array}$ & N/A & On stems and leaves of Crotalaria medicaginea & India & Pavgi \& Singh (1965) \\
\hline $\begin{array}{l}\text { C. gardeniarum Pavgi \& } \\
\text { U.P. Singh }\end{array}$ & N/A & On leaves of Gardenia & India & Pavgi \& Singh (1964) \\
\hline C. geniculatum Sawada & N/A & On leaves of Piper betle & Taiwan & Sawada (1959) \\
\hline $\begin{array}{l}\text { C. georgius-fischeri } \mathrm{R} \text {. } \\
\text { Sprague }\end{array}$ & Holotype WSP 42933 & On leaves of Muehlenbergia filiformis & US & Sprague (1958) \\
\hline
\end{tabular}


Table 19 Continued.

\begin{tabular}{|c|c|c|c|c|}
\hline Species & Typification Details & Host-Substratum & Country & Reference \\
\hline $\begin{array}{l}\text { C. gliricidiae Syd. \& P. } \\
\text { Syd. }\end{array}$ & Collado 6988 & On living leaves of Gliricidia sepium & Philippines & Sydow \& Sydow (1920) \\
\hline C. glochidii C.C. Chen & N/A & On leaves of Glochidon hongkongense & Taiwan & Chen (1967) \\
\hline $\begin{array}{l}\text { C. glochidiicola } \\
\text { (Seshadri) B. Sutton }\end{array}$ & Holotype MACS 228 & On leaves of Glochidion hohenackeri & India & Sutton $(1975)$ \\
\hline $\begin{array}{l}\text { C. gloriosae Verpl. \& } \\
\text { Claess. }\end{array}$ & Holotype GENT, Verplancke s.n. & On leaves of Yucca gloriosa & Belgium & Verplancke \& Claessens (1934) \\
\hline $\begin{array}{l}\text { C. glycines Hori ex } \\
\text { Hemmi }\end{array}$ & N/A & On Glycine soja & Japan and Korea & Hemmi (1920) \\
\hline C. gnaphalii Syd. & Sydow 137 & On Gnaphalium spicatum & Ecuador & Sydow \& Sydow (1939) \\
\hline $\begin{array}{l}\text { C. gomphrenae A.K. } \\
\text { Srivast. }\end{array}$ & $\begin{array}{l}\text { Holotype, IARI Crypt Herb. } \\
\text { Orient } 28202 \text {, A.K. Srivastava, } \\
\text { sep } 1963\end{array}$ & On leaves of Gomphrena celosioides & India & Srivastava (1969) \\
\hline C. gossypii Southw. & N/A & On Gossypium & US & Soothworth (1891) \\
\hline C. gossypinum Edgerton & N/A & On Gossypium & US & Edgerton (1908) \\
\hline $\begin{array}{l}\text { C. griseum Heald \& F.A. } \\
\text { Wolf }\end{array}$ & $\begin{array}{l}\text { Holotype BPI, Heald \& Wolf, } \\
1280\end{array}$ & On leaves and branches of Euonymus japonicus & US & Heald \& Wolf (1911) \\
\hline C. grossulariae Jacz. & N/A & On fruits of Ribis grossularia & Russia & Jaczewski (1906) \\
\hline C. guaiaci Syd. & Sydow 213 & On living leaves of Guajacum officinale & Venezuela & Sydow (1930) \\
\hline $\begin{array}{l}\text { C. guaranicola F.C. } \\
\text { Albuq. }\end{array}$ & $\begin{array}{l}\text { Holotype IAC 8183, F.C. } \\
\text { Albuquerque, 1959-06-30 }\end{array}$ & On living leaves of Paullinia cupana var. sorbilis & Brazil & Albuquerque (1960) \\
\hline $\begin{array}{l}\text { C. guaraniticum (Speg.) } \\
\text { Speg. }\end{array}$ & Holotype LPS, Balansa 3562 & On living leaves of Sapindaceae & Paraguay & Spegazzini (1918b) \\
\hline C. gymnocladi Urries & N/A & On Gymnocladus canadensis & Spain & Urríes (1952) \\
\hline C. hederae (Pass.) Died. & N/A & On leaf spots of Hederae helicis & Italy & Diedicke (1915) \\
\hline C. helianthi Davis & Davis, 7 Jul. 1907 & On leaves of Helianthus & US & Davis (1915) \\
\hline $\begin{array}{l}\text { C. helianthicola Sousa da } \\
\text { Câmara }\end{array}$ & N/A & On Helianthus annuus & Portugal & Sousa da Câmara (1936) \\
\hline $\begin{array}{l}\text { C. helichrysi (G. Winter) } \\
\text { Arx }\end{array}$ & N/A & On living leaves of Helichrysum & South Africa & von Arx (1957a) \\
\hline $\begin{array}{l}\text { C. helicis (Desm.) } \\
\text { Morgan-Jones }\end{array}$ & N/A & N/A & N/A & Morgan-Jones (1971) \\
\hline C. heveae Petch & Holotype K(M) 167287 & On leaves of Hevea brasiliensis & Sri Lanka & Petch (1906) \\
\hline C. heynei P.C. Gupta & N/A & On leaves of Vallaris heynii & India & Gupta (1974a) \\
\hline C. hibisci Pollacci & N/A & On the stems of Hibisci palustris & Italy & Pollacci (1896) \\
\hline $\begin{array}{l}\text { C. hibisci-cannabini } \\
\text { Sawada }\end{array}$ & N/A & On leaves and stems of Hibiscus cannabinus & Taiwan & Sawada (1959) \\
\hline C. hibiscicola Rangel & N/A & On leaves of Hibisci tiliacei & Brazil & Maublanc \& Rangel (1915) \\
\hline
\end{tabular}


Table 19 Continued.

\begin{tabular}{|c|c|c|c|c|}
\hline Species & Typification Details & Host-Substratum & Country & Reference \\
\hline $\begin{array}{l}\text { C. himantophylli Kabát \& } \\
\text { Bubák }\end{array}$ & N/A & On leaves of Himantophylli miniati & Czech Republic & Kabát \& Bubák (1907) \\
\hline C. holci (P. Syd.) Grove & N/A & On living leaves of Holcus lanatus & Germany & Grove (1918) \\
\hline C. holopteleae Hasija & N/A & On leaves of Holoptelea integrifolia & India & Hasija (1962c) \\
\hline C. hortorum Av.-Saccá & N/A & $\begin{array}{l}\text { On fruit of Pimenta officinalis and Solanum } \\
\text { melongena }\end{array}$ & Brazil & Averna-Saccá (1917) \\
\hline C. humuli Dearn. & N/A & On living leaves of Humulus lupulus & Canada & Bisby et al. (1929) \\
\hline C. hydrangeae Sawada & N/A & On Hydrangea chinensis & Taiwan & Sawada (1965) \\
\hline $\begin{array}{l}\text { C. hysteriiforme }(\text { Peck) } \\
\text { H.C. Greene }\end{array}$ & Peck s.n. & On dead stems of Caulophyllum thalictroides & US & Greene (1950) \\
\hline C. idaeinum Syd. & N/A & On dead twigs of Rubus idaeus & Germany & Sydow (1932) \\
\hline $\begin{array}{l}\text { C. imantophylli Verpl. \& } \\
\text { Claess. }\end{array}$ & N/A & On leaves of Clivia nobilis & Belgium & N/A \\
\hline C. imperatae Politis & N/A & On leaves of Imperata cylindrical & Greece & Politis (1935) \\
\hline C. inamardii Lal & N/A & On Carissa carandas & India & Lal (1953) \\
\hline C. incarnatum Zimm. & N/A & On stems of Coffea liberiacae & Java & Zimmermann (1901) \\
\hline C. indicum Dastur & N/A & On living branch of Gossypium & India & Dastur (1934) \\
\hline C. indigoferae Sawada & N/A & On stems of Indigofera tinctoria & Taiwan & Sawada (1959) \\
\hline $\begin{array}{l}\text { C. intermedium (Sacc.) } \\
\text { Sawada }\end{array}$ & Roumeguère s.n. & On dead leaves of Citrus aurantium & France & Sawada (1959) \\
\hline $\begin{array}{l}\text { C. ipomoeae Sousa da } \\
\text { Câmara }\end{array}$ & N/A & On stems of Ipomoea batatas & Portugal & Sousa da Câmara (1931a) \\
\hline C. ipomoeicola V. Rao & N/A & On leaves of Ipomoea batatas & India & Rao (1963) \\
\hline C. iresines F. Stevens & Holotype ILL, Stevens 139 & On leaves of Iresine & Costa Rica & Stevens $(1927)$ \\
\hline $\begin{array}{l}\text { C. iridis Kuhnh.-Lord. \& } \\
\text { J.P. Barry }\end{array}$ & N/A & On Iris spuria subsp. maritime & France & $\begin{array}{l}\text { Kuhnholtz-Lordat \& Barry } \\
\text { (1949) }\end{array}$ \\
\hline $\begin{array}{l}\text { C. ixorae Griffon \& } \\
\text { Maubl. }\end{array}$ & $\mathrm{T}$ & On leaves of Ixora stricta & France & Griffon \& Maublang (1909) \\
\hline $\begin{array}{l}\text { C. ixorae-parviflorae } \\
\text { Patw. \& Sathe }\end{array}$ & N/A & On leaves of Ixora parviflora & India & Patwardhan \& Sathe (1965) \\
\hline C. jahnii Syd. & Sydow 111 & On living leaves of Pithecellobium dulce & Venezuela & Sydow (1930) \\
\hline C. janczewskii Namysł. & $\begin{array}{l}\text { 1. KRAM F-8196, B. } \\
\text { Namyslowski2. KRAM F-8197 }\end{array}$ & On stems and leaves Poea trivialis & Poland & Namyslowski (1906) \\
\hline $\begin{array}{l}\text { C. janiphae (Thüm.) } \\
\text { Grove }\end{array}$ & N/A & On dead stalks of Jatrophae janiphae & Australia & Grove (1919) \\
\hline C. jasminicola Tilak & N/A & On leaves and culms of Jasminum sambac & US & Tilak (1960) \\
\hline $\begin{array}{l}\text { C. jatrophae (Speg.) E.E. } \\
\text { Butler \& Bisby }\end{array}$ & Spegazzini, Apr. 1906 & On leaves of Jatropha macrocarpa & Argentina & Butler \& Bisby (1931) \\
\hline
\end{tabular}


Table 19 Continued.

\begin{tabular}{|c|c|c|c|c|}
\hline Species & Typification Details & Host-Substratum & Country & Reference \\
\hline C. jussiaeae Earle & Atkinson, 27 Aug. 1891 & On living leaves of Jussiaea decurrens & US & Earle (1897) \\
\hline C. kaki Maffei & N/A & On living leaves of Diospyros kaki var. kiombo & Italy & Maffei (1921) \\
\hline $\begin{array}{l}\text { C. kawakamii (Miyabe) } \\
\text { Sawada }\end{array}$ & N/A & On Paulowniae tomentosae & Japan & Sawada (1958) \\
\hline $\begin{array}{l}\text { C. kickxiae Henn. ex } \\
\text { Died. }\end{array}$ & N/A & On Kickxia elastica & Germany & Diedicke (1915) \\
\hline $\begin{array}{l}\text { C. kiotoense (Hemmi \& } \\
\text { Nojima) Petr. }\end{array}$ & N/A & On dead branch of Aucuba japonica & Japan & Petrak (1939) \\
\hline $\begin{array}{l}\text { C. kruegerianum } \\
\text { Vassiljevsky }\end{array}$ & N/A & On fruit of Lycopersicon esculentum & US & $\begin{array}{l}\text { Vassiljevsky \& Karakulin } \\
\text { (1950) }\end{array}$ \\
\hline $\begin{array}{l}\text { C. lagenaria (Pass.) Ellis } \\
\& \text { Halst. }\end{array}$ & N/A & On Cucumeris melonis & Japan & Halsted (1893b) \\
\hline $\begin{array}{l}\text { C. latium J.Yi Li \& X.M. } \\
\text { Wang }\end{array}$ & N/A & On petiole of Ricinus communis & China & Wang \& Li 1987 \\
\hline $\begin{array}{l}\text { C. lebbek (Syd. \& P. } \\
\text { Syd.) Petr. }\end{array}$ & Merrill S149 & On legumes of Albizia lebbek & Philippines & Petrak (1927) \\
\hline $\begin{array}{l}\text { C. leguminis (Cooke \& } \\
\text { Harkn.) Negru }\end{array}$ & Harkness 1203 & On Leguminosae & US & Negru (1959) \\
\hline C. leguminum Pat. & N/A & On Pisi sativum & Italy & Patouillard \& Lagerheim (1891) \\
\hline C. ligustri Lobik & $\mathrm{N} / \mathrm{A}$ & On leaves of Ligustrum vulgare & Russia & Lobik (1928) \\
\hline C. liliacearum Ferraris & N/A & On leaves of Hemerocallis fulva & Italy & Duke (1928) \\
\hline $\begin{array}{l}\text { C. litchi Gonz. Frag. \& } \\
\text { Cif. }\end{array}$ & N/A & On leaves of Litchi sinensis & $\begin{array}{l}\text { Dominican } \\
\text { Republic }\end{array}$ & González \& Ciferri (1927) \\
\hline C. littoralis Av.-Saccá & N/A & On living roots and stems of Solanum tuberosum & Brazil & Averna-Saccá (1923) \\
\hline C. liukiuensis Sawada & N/A & On leaves of Musa liukiuensis & Taiwan & Sawada (1959) \\
\hline C. lobeliae F. Stevens & ILL, Stevens 776 & On leaves of Lobelia assurgens var. portoricensis & Brazil & Stevens (1917) \\
\hline C. lonicerae Politis & N/A & On living leaves of Lonicera etrusca & Greece & Politis (1935) \\
\hline C. lucidae H.C. Greene & Greene, 4 Sept. 1955 & On leaves of Salix lucida & US & Greene (1956) \\
\hline C. lucumae Novoss. & N/A & On Lucuma sellowii in greenhouses & Russia & $\begin{array}{l}\text { Bondartseva-Monteverde et al. } \\
\text { (1936) }\end{array}$ \\
\hline C. lujae Verpl. \& Clem. & N/A & N/A & N/A & Saccardo (1914) \\
\hline C. lussoniense Sacc. & Raimundo 1613 & On dead branches of Manihot utilissima & Phillipines & Hall \& Drost (1908) \\
\hline $\begin{array}{l}\text { C. luxificum C.J.J. Hall \& } \\
\text { Drost }\end{array}$ & N/A & On shoots and berries of Theobroma cacao & Indonesia & Hall \& Drost (1908) \\
\hline $\begin{array}{l}\text { C. luzulae Togashi \& } \\
\text { Onuma }\end{array}$ & N/A & On living leaves of Luzula campestris var. capitata & Japan & Togashi \& Onuma (1934) \\
\hline
\end{tabular}


Table 19 Continued.

\begin{tabular}{|c|c|c|c|c|}
\hline Species & Typification Details & Host-Substratum & Country & Reference \\
\hline C. lysimachiae Duke & $\begin{array}{l}\text { Duke, } 20 \text { Sept. } 1925 \text {, Duke, } 29 \\
\text { Sept. } 1926 \text {, Duke, } 23 \text { Oct. } 1926\end{array}$ & On leaves, stems and fruit of Lysimachia nemorum & Ireland and UK & Duke (1928) \\
\hline C. maculans G. Winter & N/A & On Caulibus herbarum & Germany & Dickson (1925) \\
\hline $\begin{array}{l}\text { C. madisonense H.C. } \\
\text { Greene }\end{array}$ & Greene, 25 Jun. 1952 & On Carex lacustris & US & Greene $(1953 b)$ \\
\hline $\begin{array}{l}\text { C. magnoliae Sousa da } \\
\text { Câmara }\end{array}$ & N/A & On leaves of Magnoliae grandiflorae & Portugal & Sousa da Câmara (1920) \\
\hline C. magnusianum Bres. & N/A & On leaves of Malvae neglectae & Italy & Bresadola (1892) \\
\hline C. mahoniae Fabric. & N/A & On Mahonia aquifolium & Italy & Fabricatore (1950) \\
\hline C. mali Woron. & N/A & On fallen fruit of Piri mali & German & Woronichin (1913) \\
\hline $\begin{array}{l}\text { C. malvacearum Pavgi \& } \\
\text { U.P. Singh }\end{array}$ & N/A & On leaves of Hibiscus rosa-sinensis & India & Pavgi \& Singh (1965) \\
\hline C. mangenotii Chevaug. & N/A & On Arachis hypogaea & Sudan & Chevaugeon (1952) \\
\hline C. mangiferae Kelkar & Holotype MACS 101 & On leaves of Mangifera indica & India & Kelkar \& Rao (1962) \\
\hline C. manihoticola Sawada & N/A & On dead stems of Manihot utilissima & Taiwan & Sawada K (1959) \\
\hline C. manihotis Henn. & Ule 2632 & On living leaves of Manihot utilissima & Brazil & Hennings (1904b) \\
\hline C. marantae Sawada & N/A & On leaves of Maranta arundinacea & Taiwan & Sawada (1959) \\
\hline C. martyniae V. Rao & Holotype MACS 105 & On leaves of Martynia diandra & India & Kelkar \& Rao (1962) \\
\hline C. mathiolae Sandu & N/A & On leaves of Matthiola incana & Romania & Sandu $(1959 b)$ \\
\hline $\begin{array}{l}\text { C. medicaginis- } \\
\text { denticulatae Sawada }\end{array}$ & N/A & On leaves of Medicago denticulata & Taiwan & Sawada (1933) \\
\hline C. medinillae Rangel & N/A & On leaves of Medinillae magnificae & Brazil & Maublanc \& Rangel (1915) \\
\hline $\begin{array}{l}\text { C. melastomacearum } \\
\text { (Speg.) Syd. }\end{array}$ & Holotype LPS, Puiggar 2756 & On living leaves of Melastomataceae & Brazil & Sydow $(1925 b)$ \\
\hline $\begin{array}{l}\text { C. meliae Sousa da } \\
\text { Câmara }\end{array}$ & N/A & On stems of Meliae azedarch & Portugal & Sousa da Câmara (1920) \\
\hline C. melicoccae Speg. & Spegazzini, Oct. 1920 & On living leaves of Melicoccus lepidopetalus & Paraguay & Spegazzini (1922) \\
\hline C. melongenae Av.-Saccá & $\mathrm{N} / \mathrm{A}$ & On fruit of Solanum melongena & Brazil & Averna-Saccá (1917) \\
\hline $\begin{array}{l}\text { C. memecyli Narendra \& } \\
\text { V.G. Rao }\end{array}$ & Holotype MACS 1596 & On leaves of Memecylon umbellatum & India & Narendra \& Rao (1972) \\
\hline $\begin{array}{l}\text { C. merilli (Syd. \& P. } \\
\text { Syd.) Quimio }\end{array}$ & Holotype PNH 21350 & On stems of Ricinus communis & Philippines & Quimio (1977) \\
\hline C. microspermum Corda & N/A & N/A & N/A & Corda $(1840)$ \\
\hline C. minus Zimm & N/A & On leaves of Palaquii oblongifolii & Jarva & Zimmermann (1901) \\
\hline $\begin{array}{l}\text { C. minutum (Link) B.T. } \\
\text { Dicks }\end{array}$ & N/A & On Caulibus herbarum & Germany & Dickson (1925) \\
\hline $\begin{array}{l}\text { C. mirabile (Bres.) S. } \\
\text { Ahmad }\end{array}$ & Torrend 707 & On stems of Mirabilis jalapa & Portugal & Ahmad (1956) \\
\hline
\end{tabular}


Table 19 Continued.

\begin{tabular}{|c|c|c|c|c|}
\hline Species & Typification Details & Host-Substratum & Country & Reference \\
\hline $\begin{array}{l}\text { C. moellerianum (Thüm.) } \\
\text { Vassiljevsky }\end{array}$ & N/A & On decaying stems Phytolacca decandra & Italy & Vassiljevsky (1940) \\
\hline C. montemartinii Tognini & N/A & On leaves of Arum italicum & Italy & Tognini (1892) \\
\hline C. moricola Sawada & N/A & On twigs of Morus alba & Taiwan & Sawada (1959) \\
\hline C. morina Hara & N/A & On leaves of Morus alba & Japan & Hara (1954) \\
\hline C. morindae Kelkar & Holotype MACS 103 & On leaves of Morinda citrifolia & India & Kelkar \& Rao (1962) \\
\hline C. morinum Sawada & N/A & On twigs of Morus alba & Taiwan & Sawada (1959) \\
\hline C. necator Massee & Holotype K (M), Ridley s.n. & On fruit of Piper nigrum & Singapore & Massee (1912) \\
\hline $\begin{array}{l}\text { C. neriicola Hüseyın \& } \\
\text { Selçuk }\end{array}$ & Holotype IU, Hüseyin 0506 & On leaves of cultivated Nerium oleander & Turkey & Hüseyin \& Selçuk (2001) \\
\hline C. nicotianae Av.-Saccá & N/A & On Nicotiana tabacum & Brazil & Averna-Saccá (1922) \\
\hline $\begin{array}{l}\text { C. nobile (Sacc.) } \\
\text { Mzhavan. }\end{array}$ & Thümen, Sept. 1879 & On leaves of Laurus nobilis & Austria & Mzhavanadze (1963) \\
\hline C. nymphaeicola Kelkar & N/A & On leaves of Nymphaea stellata & India & Kelkar (1972) \\
\hline $\begin{array}{l}\text { C. obtusipes (Sacc.) P. } \\
\text { Joly }\end{array}$ & N/A & On young stems of Bauhinia glandulosa & Italy & Joly (1965) \\
\hline C. oca M.L. Farr & N/A & On leaves of Oxalis tuberosa & Bolivia & Farr (1960) \\
\hline C. olacicola Muthappa & $\begin{array}{l}\text { HCIO, Muthappa, MACS274, } \\
\text { IMI, Muthappa, MACS274 }\end{array}$ & On leaves of Olax wightiana & India & Muthappa (1967) \\
\hline C. oleae Kelkar & Holotype MACS 104 & On leaves of Olea dioica & India & Kelkar \& Rao (1962) \\
\hline C. oligochaetum Cavara & N/A & On young leaves of Lagenaria vulgaris & Italy & Cavara $(1889 b)$ \\
\hline $\begin{array}{l}\text { C. oligotrichum Dias \& } \\
\text { Sousa da Câmara }\end{array}$ & N/A & On Passiflora coerulea & Portugal & Dias \& Sousa da Câmara (1952) \\
\hline C. omnivorum Halst. & N/A & On leaves and petioles of Aspirustra & US & Halsted (1891) \\
\hline C. ophiopogonis Sandu & $\mathrm{N} / \mathrm{A}$ & On dead leaves of Ophiopogon jabura & Romania & Sandu-Ville (1962) \\
\hline $\begin{array}{l}\text { C. opuntiae (Ellis \& } \\
\text { Everh.) Sawada }\end{array}$ & NY, Demetrio, Jan. 1886 & On dead Opuntia brasiliensis & US & Sawada (1959) \\
\hline C. ornithogali Negru & N/A & On leaves of bulbs of Ornithogalum longibracteatum & Romania & Negru (1958) \\
\hline C. orthianum Kostlan & N/A & $\begin{array}{l}\text { On Orchidaceae (Cypripedium, Cattleya, Coelogyne, } \\
\text { Dendrobium, Vanda) }\end{array}$ & Germany & Kostlan (1905) \\
\hline $\begin{array}{l}\text { C. orthosporum (Sacc. \& } \\
\text { Roum.) B.T. Dicks }\end{array}$ & N/A & On Solani tuberosi & Italy & Dickson (1926) \\
\hline C. oryzae (Hara) Hara & N/A & On Oryza sativa & Japan & Hara (1959) \\
\hline C. osmanthi Dzhalag. & N/A & On leaves of Osmanthus fragrans & Azerbaijan & Dzhalagoniya (1965) \\
\hline C. padi Karak. & N/A & On living leaves of Prunus padus & Russia & Karakulin (1924) \\
\hline C. palaquii Zimm. & N/A & On leaves of Palaquii oblongifolii & Java & Zimmermann (1901) \\
\hline
\end{tabular}


Table 19 Continued.

\begin{tabular}{|c|c|c|c|c|}
\hline Species & Typification Details & Host-Substratum & Country & Reference \\
\hline $\begin{array}{l}\text { C. palinhae González } \\
\text { Fragoso }\end{array}$ & N/A & $\begin{array}{l}\text { On branches of Grevillea robusta and Lamproderma } \\
\text { echinulatum }\end{array}$ & Portugal & González (1924) \\
\hline $\begin{array}{l}\text { C. paludosum (Ellis \& } \\
\text { Galloway) Arx }\end{array}$ & $\begin{array}{l}\text { Fairchild, Aug. 1889, Commons } \\
977\end{array}$ & On leaves of Peltandra virginica & US & von Arx (1957a) \\
\hline C. pamparum Speg. & Spegazzini, May 1908 & On leaves of Panicum urvilleani & Argentina & Spegazzini (1911) \\
\hline C. pancratii Hasija & N/A & On leaves of Pancratium & India & Hasija (1962a) \\
\hline $\begin{array}{l}\text { C. pandani Syd. \& P. } \\
\text { Syd. }\end{array}$ & Merrill S33 & On leaves of Pandanus veitchii & Philippines & Sydow \& Sydow (1913) \\
\hline C. papayae Henn. & Putteman 1338 & On branches and petioles of Carica papaya & Brazil & Hennings (1908a) \\
\hline $\begin{array}{l}\text { C. parthenocissi (C.I. } \\
\text { Shen) Teng }\end{array}$ & N/A & On Parthenocissus sp. & N/A & Teng (1936) \\
\hline C. passiflorae Siemaszko & $\begin{array}{l}\text { Syntype Stevens } 465=\text { BISH } \\
\text { 145782, 499913, Stevens 914= } \\
\text { BISH 499010, 508404 }\end{array}$ & On living leaves of Passiflora edulis and P. laurifolia & $\begin{array}{l}\text { Armenia \& } \\
\text { Azerbaijan, US }\end{array}$ & Siemaszko (1923) \\
\hline C. paucipilum Delacr. & N/A & N/A & N/A & Delacroix (1905) \\
\hline $\begin{array}{l}\text { C. peckianum (Thüm.) } \\
\text { Petr. \& Syd. }\end{array}$ & N/A & On decaying shoots of Vitis labruscana & US & Petrak \& Sydow (1924) \\
\hline C. peckii (Sacc.) Davis & N/A & On leaves of Trillii erythrocarpi & US & Davis (1929) \\
\hline C. pekinense Katsura & N/A & On Abutilon avicennae & China & Katsura (1951) \\
\hline C. peregrinum Pass. & $\mathrm{N} / \mathrm{A}$ & On leaves of Araliae sieboldii & Italy & Passerini (1888) \\
\hline $\begin{array}{l}\text { C. periclymeni (Desm.) } \\
\text { Höhn. }\end{array}$ & N/A & On leaves of Lonicerae periclymeni & France & Höhnel (1957) \\
\hline $\begin{array}{l}\text { C. petiolicola (Brunaud) } \\
\text { Grove }\end{array}$ & N/A & On fallen petioles of Ailanthi glandulosae & Gaul region & Grove (1918) \\
\hline $\begin{array}{l}\text { C. phalaenopsidis } \\
\text { Sawada }\end{array}$ & N/A & On leaves of Phalaenopsis aphrodite & Taiwan & Sawada (1959) \\
\hline C. philodendri Henn. & Ule 3053 & On leaves of Philodendron & US & Hennings (1905b) \\
\hline $\begin{array}{l}\text { C. phomoides (Sacc.) } \\
\text { Chester }\end{array}$ & N/A & On fruit epicarp of Lycopersici esculenti & Italy & Chester (1894) \\
\hline $\begin{array}{l}\text { C. phyllachoroides (Ellis } \\
\text { \& Everh.) Arx }\end{array}$ & NY, McClatchie 371 & On leaves of Artemisia vulgaris var. californica & US & von $\operatorname{Arx}(1957 a)$ \\
\hline $\begin{array}{l}\text { C. phyllocacti Ellis \& } \\
\text { Everh. }\end{array}$ & NY, Carver s.n & On Phyllocactus latifrons & US & Ellis \& Everhart (1902) \\
\hline C. pilcomayense Speg. & Spegazzini, Jul. 1919 & On leaves of Dolychandra cynanchoidis & Paraguay & Spegazzini (1922) \\
\hline C. pinelliae Sawada & N/A & On Pinellia ternata & Taiwan & Sawada (1943) \\
\hline $\begin{array}{l}\text { C. piperatum Ellis \& } \\
\text { Everh. }\end{array}$ & N/A & On leaves of Capsici annui & US & N/A \\
\hline C. pithecellobii Roldan & N/A & On Pithecolobium dulce & Philippines & Roldan (1936) \\
\hline
\end{tabular}


Table 19 Continued.

\begin{tabular}{|c|c|c|c|c|}
\hline Species & Typification Details & Host-Substratum & Country & Reference \\
\hline C. plantaginis Sawada & N/A & On Plantago sawadai & Taiwan & Sawada (1943) \\
\hline $\begin{array}{l}\text { C. platani Sousa da } \\
\text { Câmara }\end{array}$ & N/A & On petioles of Platani orientalis & Portugal & Sousa da Câmara (1910) \\
\hline $\begin{array}{l}\text { C. poinsettiae (Sacc.) } \\
\text { Petr. }\end{array}$ & N/A & On stems of Poinsettiae pelecherrimae & Gaul region & Ahmad (1956) \\
\hline C. pollaccii Magnaghi & N/A & On leaves of Aucubae japonicae & Pegli, Italy & Magnaghi (1902) \\
\hline $\begin{array}{l}\text { C. polyptychophyllum } \\
\text { Sousa da Câmara }\end{array}$ & N/A & On leaves of Rhododendron & Portugal & Sousa da Câmara (1931b) \\
\hline C. pothi Koord. & N/A & On leaves of Pothi & Java & Koorders (1907) \\
\hline C. primulae Halst. & N/A & On living leaves of Primula sp. & US & Saccardo \& Sydow (1899) \\
\hline $\begin{array}{l}\text { C. pruni-domesticae } \\
\text { Gurz. }\end{array}$ & $\begin{array}{l}\text { CBS H-10669, A. Negru, 1953- } \\
08-20\end{array}$ & On living leaves of Prunus domestica & Romania & Gurzitska (1950) \\
\hline C. prunicola H.C. Greene & N/A & On leaves of Prunus serotina & US & Greene (1951) \\
\hline C. psoraleae (Peck) Arx & $\begin{array}{l}\text { CBS H-4241, E. Bartolomew, } \\
\text { 1892-06-20 }\end{array}$ & On leaves of Psoralea esculenta & US & von Arx (1957a) \\
\hline C. pterocelastri Wakef. & Van der Bjl 404 & On living leaves of Pterocelastrus variabilis & South Africa & Wakefield (1922) \\
\hline $\begin{array}{l}\text { C. pucciniophilum } \\
\text { Togashi }\end{array}$ & N/A & On Hosta sieboldiana & Japan & Togashi (1936) \\
\hline C. pyri F. Noack & N/A & On leaves of Piri mali & Brazil & Noack (1897) \\
\hline $\begin{array}{l}\text { C. pyrolae (Trel.) } \\
\text { Parmelee }\end{array}$ & colln Trelease s.n. & On leaves of Pyrola rotundifolia & US & Parmelee (1958) \\
\hline $\begin{array}{l}\text { C. quercinum Săvul. \& } \\
\text { Negru }\end{array}$ & $\begin{array}{l}\text { CBS H-4821, A. Negru, 1951- } \\
09-10\end{array}$ & On leaves of Quercus sessiliflora & Romania & Savulescu \& Negru (1955) \\
\hline C. rayssiae Massenot & N/A & On leaves of Asarum europaeum & France & Massenot (1961) \\
\hline $\begin{array}{l}\text { C. revolutum (Ellis \& } \\
\text { Everhart) K. Ito \& } \\
\text { Kobayasi }\end{array}$ & NY, Ellis \& Everhart, Aug. 1889 & On living leaves of Robinia pseudoacacia & US & Ito \& Kobayashi (1958) \\
\hline $\begin{array}{l}\text { C. rhamni E. Rădul. \& } \\
\text { Negru }\end{array}$ & N/A & On leaves of Rhamnus tinctoria and $R$. infectoria & Romania & Radulescu \& Negru (1963) \\
\hline $\begin{array}{l}\text { C. rhodocyclum } \\
\text { (Montagne ex Almeida \& } \\
\text { Sousa da Camara) Petrak }\end{array}$ & N/A & On leaves of Phormium tenax & Portugal & Petrak (1927) \\
\hline C. rhoinum Tassi & N/A & On leaves of Rhois toxicodendri & Italy & Tassi (1899) \\
\hline $\begin{array}{l}\text { C. rhois-verniciferae } \\
\text { Elisei }\end{array}$ & N/A & On Rhus vernicifera & Italy & Elisei (1938) \\
\hline $\begin{array}{l}\text { C. rhynchosiae Pavgi \& } \\
\text { U.P. Singh }\end{array}$ & N/A & On leaves of Rhynchosia minima & India & Pavgi \& Singh (1964) \\
\hline
\end{tabular}


Table 19 Continued.

\begin{tabular}{|c|c|c|c|c|}
\hline Species & Typification Details & Host-Substratum & Country & Reference \\
\hline $\begin{array}{l}\text { C. ricini Bubák \& } \\
\text { González Fragoso }\end{array}$ & N/A & On stalk of Ricinus communis & Sri Lanka & Bubák \& González (1915) \\
\hline C. rosarum Av.-Saccá & N/A & On leaves of Rosa sp. & Brazil & $\begin{array}{l}\text { Vassiljevsky \& Karakulin } \\
\text { (1926) }\end{array}$ \\
\hline C. roseolum Henn. & Behnick, Dec. 1904 & On dead bulbs of Stanhopea oculata & Germany & Hennings (1905a) \\
\hline $\begin{array}{l}\text { C. rubi Dias \& Sousa da } \\
\text { Câmara }\end{array}$ & N/A & On Rubus idaeus & Portugal & Dias \& Sousa da Câmara (1953) \\
\hline C. rubicola Ellis \& Everh. & N/A & On leaves of Rubi strigosi & US & Peck (1902) \\
\hline C. rudbeckiae Peck & Stewart s.n. & On dead stems of Rudbeckia laciniata & US & Peck (1902) \\
\hline C. rumicicola Sawada & N/A & On leaves of Rumex japonicus & Japan & Sawada (1958) \\
\hline C. rumicis-crispi Sawada & N/A & On leaves of Rumex crispus & Taiwan & Sawada (1959) \\
\hline C. salmonicolor O'Gara & N/A & On the stems and leaves of Asclepiadis speciose & US & O’Gara (1915) \\
\hline $\begin{array}{l}\text { C. samararum (Allesch.) } \\
\text { Petr. }\end{array}$ & N/A & On the fallen fruit of Fraxini excelsioris & Germany & Petrak (1956) \\
\hline C. sanguisorbae Bres. & N/A & On leaves of Sanguisorbae officinalis & Limes Saxoniae & Bresadola (1894) \\
\hline $\begin{array}{l}\text { C. sapindi Sousa da } \\
\text { Câmara }\end{array}$ & N/A & On Sapindus saponaria & Portugal & Sousa da Câmara (1936) \\
\hline $\begin{array}{l}\text { C. sasicola } \text { I. Hino \& } \\
\text { Katum. }\end{array}$ & N/A & On culms of Sasa kurilensis & Japan & Hino \& Katumoto (1958) \\
\hline C. sativum N.L. Horn & N/A & On Vicia sativa & US & Horn (1952) \\
\hline C. savulescui Sandu & N/A & On leaves and fruit of Lens culinaris & Romania & Sandu $(1959 a)$ \\
\hline $\begin{array}{l}\text { C. schizanthi C.N. Jensen } \\
\text { \& V.B. Stewart }\end{array}$ & N/A & $\begin{array}{l}\text { On the stems, branches, leaves and petioles of } \\
\text { Schizanthi sp. }\end{array}$ & US & Jensen \& Stewart (1911) \\
\hline $\begin{array}{l}\text { C. seguieriae Bond.- } \\
\text { Mont. }\end{array}$ & N/A & On Seguieria americana in greenhouses & Russia & $\begin{array}{l}\text { Bondartseva-Monteverde et al. } \\
\text { (1936) }\end{array}$ \\
\hline $\begin{array}{l}\text { C. seminicola (Berk. \& } \\
\text { Broome) Petch }\end{array}$ & $\begin{array}{l}\text { Holotype K(M), Gardner 1132, } \\
\text { Dec. } 1868\end{array}$ & On seeds of Artocarpus integrifolia & Sri Lanka & Petch (1924) \\
\hline C. septorioides Sacc. & N/A & On dead culms of Bambusae vulgaris & China & Saccardo (1921) \\
\hline C. servazzii Gall.-Rang. & N/A & On leaves of Cattleya & Italy & Gallucci-Rangone (1955) \\
\hline $\begin{array}{l}\text { C. sesbaniae Pavgi \& } \\
\text { U.P. Singh }\end{array}$ & N/A & On stems and leaflets of Sesbania aegyptiaca & India & Pavgi \& Singh (1965) \\
\hline C. setosum F. Patt. & Holotype BPI, Werckle, 1899 & On Tillandsia & Costa Rica & Patterson (1900) \\
\hline C. sierraense R. Sprague & Holotype WSP 42900 & On leaves of Poa bolanderi & US & Sprague (1958) \\
\hline C. silphii Davis & Davis, 9 Sept. 1915 & On leaves of Silphium perfoliatum & US & Davis (1919) \\
\hline C. sinuatisetiferum & Holotype Matsushima Fungus & On decaying leaf of broad-leaved tree & Japan & Matsushima (1996) \\
\hline Matsush. & Collection, Kobe, $5 \mathrm{H} 248$ & & & \\
\hline C. smilacicola Speg. & Spegazzini, Jul. 1919 & On leaves of Smilax assumptionis & Paraguay & Spegazzini (1922) \\
\hline
\end{tabular}


Table 19 Continued.

\begin{tabular}{|c|c|c|c|c|}
\hline Species & Typification Details & Host-Substratum & Country & Reference \\
\hline $\begin{array}{l}\text { C. smilacinae Tehon \& } \\
\text { E.Y. Daniels }\end{array}$ & Holotype ILL 7259 & On leaves of Smilacina racemosa & US & Tehon \& Daniels (1925) \\
\hline C. smilacis Tehon & Holotype ILL 22849 & On living leaves of Smilax hispida & US & Tehon (1933) \\
\hline C. socium $\mathrm{Syd}$. & Starcs 118 & On living leaves of Salix caprea & Latvia & Sydow (1935) \\
\hline C. solanicola O'Gara & N/A & On Solani tuberosi & US & O'Gara (1915) \\
\hline C. solidaginis Pellic. & Holotype MPA, Chevassut 2196 & On leaves of Solidago virgaurea & France & Chevassut \& Pellicier (2001) \\
\hline $\begin{array}{l}\text { C. solitarium Ellis \& } \\
\text { Barthol. }\end{array}$ & $\begin{array}{l}\text { Holotype NY, Bartholomew } \\
2426\end{array}$ & On leaves of Solidago radula & US & Ellis \& Everhart (1897) \\
\hline $\begin{array}{l}\text { C. sophorae-japonicae } \\
\text { Hara }\end{array}$ & N/A & On branchlets Sophora japonica & Japan & Hara (1930) \\
\hline $\begin{array}{l}\text { C. sorauerianum } \\
\text { (Allesch.) Hori }\end{array}$ & N/A & On leaves of Codiaeum & US & Hori (1919) \\
\hline C. sordidum Davis & Davis, 31 Jul. 1914 & On leaves of Menispermum canadense & US & Davis (1915) \\
\hline C. spartii Gutner & N/A & On living branchlets Spartium junceum & Russia & Gutner (1933b) \\
\hline C. sphaeriiforme Pass. & N/A & On dead branches of Menispermum canadense & Italy & Passerini (1888) \\
\hline C. stanhopeae Henn. & Baker 245 & On living leaves of Stanhopea & Brazil & Hennings (1908b) \\
\hline C. staphyleae Hulea & $\begin{array}{l}\text { CBS H-6941, A. Hulea, 1948- } \\
\text { 08-04 }\end{array}$ & On leaves of Staphylea pinnata & Romania & Hulea (1950) \\
\hline C. stephaniae Sawada & N/A & On leaves of Stephania sasakii & Taiwan & Sawada (1959) \\
\hline C. sterculiicola Speg. & Spegazzini, Jul. 1919 & On leaves Sterculia striata & Paraguay & Spegazzini (1922) \\
\hline C. stevensii Roy bis & Holotype ILL, Stevens 288 & On leaves of Piper umbellatum & Puerto Rico & Roy (1948) \\
\hline $\begin{array}{l}\text { C. sumbaviae Syd. \& P. } \\
\text { Syd. }\end{array}$ & Holotype PNH S258 & On living leaves of Sumbavia rottleroides & Philippines & Sydow \& Sydow (1914b) \\
\hline C. suttonii W.P. Wu & $\begin{array}{l}\text { Holotype Hebei Academy of } \\
\text { Sciences, } 910123,910124\end{array}$ & On Euonymus japonicus & China & $\mathrm{Wu}(1992)$ \\
\hline $\begin{array}{l}\text { C. tabernaemontanae } \\
\text { Pavgi }\end{array}$ & N/A & On leaves of Tabernaemontana coronaria & India & Pavgi \& Upadhyay (1964) \\
\hline $\begin{array}{l}\text { C. tabificum (Hallier) } \\
\text { Pethybr. }\end{array}$ & $\mathrm{T} \#$ & On Solanum tuberosum & Germany & Pethybridge (1919) \\
\hline $\begin{array}{l}\text { C. taiwanense Sivan. \& } \\
\text { W.H. Hsieh }\end{array}$ & T, IMI 353024b, 1992-01-13 & On stems of Styrax formosanus & Taiwan & Sivanesan \& Hsieh (1993) \\
\hline C. telles-palhinhae Dias & $\mathrm{T} \#$ & On leaves of Nerium oleander & Portugal & Sousa-Dias (1958) \\
\hline $\begin{array}{l}\text { C. teramnicola Pavgi \& } \\
\text { U.P. Singh }\end{array}$ & N/A & On leaves of Teramnus labialis & India & Pavgi \& Singh (1965) \\
\hline C. terminaliae V.P. Sahni & T, IMI 108221, 1964-08 & On leaves of Terminalia bellirica & India & Sahni (1968) \\
\hline C. tertium (Cooke) Grove & $\mathrm{T}$, Ravenel 2712 & On branches of Ailanthus & US & Grove (1919) \\
\hline $\begin{array}{l}\text { C. tetrastigmatis (Keissl.) } \\
\text { Petr. }\end{array}$ & $\mathrm{T}^{*}$, Handel-Mazzetti, 5860 & On living leaves of Tetrastigma dubium & China & Petrak (1947a) \\
\hline
\end{tabular}


Table 19 Continued.

\begin{tabular}{|c|c|c|c|c|}
\hline Species & Typification Details & Host-Substratum & Country & Reference \\
\hline C. tinosporae syd. & $\mathrm{T}^{*}$, Mitter 41 & On dead stems of Tinospora cordifolia & India & Sydow \& Mitter (1933) \\
\hline $\begin{array}{l}\text { C. toluiferae F. Stevens \& } \\
\text { Solheim }\end{array}$ & $\begin{array}{l}\text { T, ILL, Stevens 820, August 8, } \\
1922\end{array}$ & On leaves of Toluifera sp. & $\begin{array}{l}\text { Trinidad and } \\
\text { Tobago }\end{array}$ & Stevens (1930) \\
\hline $\begin{array}{l}\text { C. trevesiae (Keissler) } \\
\text { Petrak }\end{array}$ & T*, Handel-Mazzetti, 5792 & On living leaves of Trevesia palmata & China & Petrak (1947) \\
\hline C. trichiliae Novoss & $\mathrm{T} \#$ & On Trichilia undulatifolia in greenhouses & Russia & $\begin{array}{l}\text { Bondartseva-Monteverde et al. } \\
\text { (1936) }\end{array}$ \\
\hline C. tricyrtii (Teng) Teng & N/A & On living leaves of Tricyrtis & China & Teng (1936) \\
\hline C. trillii Tehon & $\begin{array}{l}\text { T, ILL 2350, Ryder, June 23, } \\
1922\end{array}$ & On living leaves of Trillium recurvatum & US & Tehon (1924) \\
\hline C. typhae H.C. Greene & T*, Greene, 7 Aug. 1954 & On leaves of Typha latifolia & US & Greene (1955) \\
\hline $\begin{array}{l}\text { C. uncinatum (Berk. \& } \\
\text { M.A. Curtis) Duke }\end{array}$ & $\mathrm{T}^{*}$, Ravenel 4691 & On stalks of Desmodium nudiflorum & US & Duke (1928) \\
\hline C. uredinophilum Hulea & $\mathrm{T} \#$ & On Muscari botryoides & Romania & Hulea (1939) \\
\hline C. urenae Petch & $\begin{array}{l}\text { T, PDA 4679, Petch T., } \\
\text { Peradeniya, June } 1915\end{array}$ & On living leaves of Urena lobata & Sri Lanka & Petch (1917) \\
\hline C. urmilae P.C. Gupta & $\mathrm{T} \#$ & On leaves of Diospyros embryopteris & India & Gupta (1974b) \\
\hline C. urticae H.C. Greene & $\mathrm{T} \#$ & On Urtica gracilis & US & Greene (1953a) \\
\hline C. valerianae Kwashn. & $\mathrm{T} \#$ & On living leaves of Valeriana officinalis & Caucasus & Kwashnina (1928) \\
\hline C. vanillae Scalia & N/A & On leaves of Vanilla odoratae & Belgium & Pavgi \& Upadhyay (1964) \\
\hline $\begin{array}{l}\text { C. variegatum U.P. Singh } \\
\text { \& H.P. Upadhyay }\end{array}$ & N/A & On leaves of Codiaeum variegatum & India & Pavgi \& Upadhyay (1964) \\
\hline C. vassiljevskyi Negru & N/A & On fruits of Cercis siliquastrum & Romania & Negru (1961) \\
\hline $\begin{array}{l}\text { C. vermicularia Sacc. \& } \\
\text { Dearn. }\end{array}$ & T*, Dearness, Aug. 1910 & On leaves of Bromi ciliati var. leviglumis & UK & Saccardo (1912) \\
\hline C. vermicularioides Halst. & $\mathrm{T} \#$ & On leaves and stems of Linaria vulgaris & US & Halsted (1893b) \\
\hline C. veronicae Scaram. & $\mathrm{T} \#$ & On living leaves of Veronica bellidioides & Italy & Scaramella (1932) \\
\hline C. versicolor Sacc. & N/A & In the dying stalks of Bambusae viridis-glaucescentis & Portugal & Saccardo (1902) \\
\hline C. viciae Dearn. \& Overh. & $\mathrm{T}^{*}$, Orton 9335, Dearness 5627 & On living leaves of Vicia villosa & US & Dearness (1928) \\
\hline C. viciae-sativae Sawada & N/A & On leaves of Vicia sativa & Taiwan & Sawada (1959) \\
\hline C. villaresiae Gutner & $\mathrm{T} \#$ & In greenhouse on branches of Villaresia & Russia & Gutner (1933a) \\
\hline C. villosum Weimer & $\mathrm{T} \#$ & On Vicia villosa & US & Weimer (1945) \\
\hline $\begin{array}{l}\text { C. vinal (Speg.) Petr. \& } \\
\text { Syd. }\end{array}$ & T*, Spegazzini, Jan. 1901 & On living leaves of Prosopis ruscifolia & Taiwan & Petrak \& Sydow (1935) \\
\hline C. vincae Speg. & T*, Spegazzini, Jul. 1905 & On leaves of Vinca major & Argentina & Spegazzini (1911) \\
\hline C. vinosum Henn. & $\mathrm{T}^{*}$, Behnick, 10 Dec. 1904 & On bulbs of Stanhopea oculata & Germany & Hennings (1905a) \\
\hline C. violae Săvul. \& Sandu & $\begin{array}{l}\text { T*, Săvulescu \& Sandu, } 2 \text { Aug. } \\
1931\end{array}$ & On living leaves of Viola hirta & Romania & $\begin{array}{l}\text { Savulescu \& Sandu-Ville } \\
\text { (1933) }\end{array}$ \\
\hline
\end{tabular}


Table 19 Continued.

\begin{tabular}{|c|c|c|c|c|}
\hline Species & Typification Details & Host-Substratum & Country & Reference \\
\hline $\begin{array}{l}\text { C. violae-rotundifoliae } \\
\text { (Sacc.) Davis }\end{array}$ & N/A & On leaves of Viola cuculata & US & Davis (1929) \\
\hline $\begin{array}{l}\text { C. violae-tricoloris R.E. } \\
\text { Sm. }\end{array}$ & N/A & Parasitic on leaves and petals of Viola tricolor & US & Smith (1899) \\
\hline C. viticis Maire & N/A & On leaves of Viticis trifoliae & US & Maire (1913) \\
\hline C. vitis Istv. & N/A & On shoots of Vitis vinifera & Hungaria & Istvanfy (1902) \\
\hline $\begin{array}{l}\text { C. volutella Sacc. \& } \\
\text { Malbr. }\end{array}$ & N/A & On dead stems of Ulmariae & France & Saccardo (1882) \\
\hline C. wahlenbergiae Duke & $\mathrm{T} \#$ & On dead leaves Wahlenbergia & UK & Duke (1928) \\
\hline C. xanthii Halst. & $\mathrm{T} \#$ & On stems of Xanthium canadense & USA & Halsted (1893a) \\
\hline C. yaquense Petr. \& Cif. & $\mathrm{T}^{*}$, colln Ciferri 4143 & On living leaves Acanthopanax aculeatus & $\begin{array}{l}\text { Dominican } \\
\text { Republic }\end{array}$ & Petrak \& Ciferri (1932) \\
\hline C. yerbae Speg. & N/A & On drooping leaves of Ilicis paraguariensis & Argentina & Spegazzini (1908) \\
\hline C. yoshinaoi T. Fukui & N/A & On Perilla frutescens & Japan & Fukui (1925) \\
\hline C. yuccae Pollacci & N/A & On leaves of Yuccae filamentosae & Italy & Pollacci (1896) \\
\hline C. zeae Lobik & $\mathrm{T} \#$ & On Zea mays & Caucasus & Lobik (1933) \\
\hline $\begin{array}{l}\text { C. zibethinum (Sacc.) } \\
\text { Petr. }\end{array}$ & N/A & On diseased leaves Durionis zibethinae & Singapore & Petrak (1929) \\
\hline
\end{tabular}

\# No details available regarding herbarium/collector/collection date

* No details available regarding herbarium

\section{Acknowledgements}

We would like to thank the National Research Council of Thailand, grant for new researcher NRCT5-TRG630010-01, entitled "Biodiversity, taxonomy, phylogeny and evolution of Colletotrichum in northern Thailand". Kevin D Hyde thanks, Thailand Research Fund, grant RDG6130001 entitled "Impact of climate change on fungal diversity and biogeography in the Greater Mekong Subregion". He would also like to thank Chiang Mai University for the award of a Visiting Professor. R.S. Jayawardena would like to thank Y.J. Chen, A.J. Gajanayake, N. Huanraluek and K.C. Mallikarathna for the support that they gave to make this work a success.

\section{References}

Abang MM, Winter S, Green KR, Hoffmann P et al. 2002 - Molecular identification of Colletotrichum gloeosporioides causing yam anthracnose in Nigeria. Plant Pathology 51, 63-71.

Adaskaveg JE, Förster H. 2000 - Occurrence andmanagement of anthracnose epidemics caused by Colletotrichum species on tree fruit crops in California. In: Colletotrichum. Host Specificity, Pathology and Host-Pathogen Interaction (eds. Prusky D, Freeman S, Dickman MB). APS Press, St Paul, Minnesota: 317-336. 
Adhikari KN, Thomas G, Buirchell BJ, Sweetingham M. 2011 - Identification of anthracnose resistance in yellow lupin (Lupinus luteus L.) and its incorporation into breeding lines. Plant Breeding 130, 660-664.

Agrios GN. 2005 - Plant pathology, $5^{\text {th }}$ edition, Academic Press, Florida, US.

Ahmad S. 1956 - Fungi of West Pakistan. Monographic Biological Society of Pakistan. 1, 1-126.

Albuquerque FC. 1960 - Antracnose do Guaraná. Boletim Tecnico do Instituto Agronomico do Norte. 40, 3-37.

Aldaoud R, de Alwis S, Salib S, Cunnington JH et al. 2011 - First record of Colletotrichum sansevieriae on Sansevieria sp. (mother - in - law's tongue) in Australia. Australasian Plant Disease Notes 6, 60-61.

Alizadeh A, Javan-Nikkhah M, Zare R, Fotouhifar KB et al. 2015 - New records of Colletotrichum species for the mycobiota of Iran. Mycologia Iranica 2, 95-109.

Allescher A. 1902 - Rabenhorst's Kryptogamen - Flora, Pilze - Fungi Imperfecti. 1, 385-704.

Ané C, Larget B, Baum DA, Smith SD, Rokas A. 2007 - Bayesian estimation of concordance among gene trees. Molecular Biology and Evolution 24, 412-426.

Arrigoni R, Berumen ML, Chen CA, Terraneo TI et al. 2016 - species delimitation in the reef coral genera Echinophyllia and Oxypora (Scleractinia, Lobophylliidae) with a description of two new species. Molecular Phylogenetics and Evolution 105, 146-159.

Arroyo FT, Moreno J, García-Herdugo G, Santos BDL et al. 2005 - Ultrastructure of the early stages of Colletotrichum acutatum infection of strawberry tissues. Canadian Journal of Botany 83, 491-500.

Auld BA, Say MM. 1999 - Comparison of isolates of Colletotrichum orbiculare from Argentina and Australia as potential bioherbicides for Xanthium spinosum in Australia. Agriculture, Ecosystems and Environment 72, 53-58.

Averna-Saccá R. 1917 - As moléstiascryptogamicas das plantashorticolas. Boletim de Agricultura São Paulo. 18, 634-654.

Averna-Saccá R. 1922 - Boletim de Agricultura São Paulo 23A, 221.

Averna-Saccá R. 1923 - Boletim de Agricultura São Paulo.

Ariyawansa HA, Hyde KD, Jayasiri SC, Buyck B et al. 2015 - Fungal diversity notes 111-252 taxonomic and phylogenetic contributions to fungal taxa. Fungal Diversity 75, 27-274.

Awadalla P. 2003 - The evolutionary genomics of pathogen recombination. Nature Reviews Genetics 4, 50-60.

Baccarini P. 1917 - Funghietiopici. Annali di Botanica. 14, 117-140.

Bailey JA, Jeger MJ. 1992 - Colletotrichum. Biology, Pathology and Control. CABI, Wallingford, UK.

Bailey JA, O'Connell RJ, Pring RJ, Nash C. 1992 - Infection strategies of Colletotrichum species. In Colletotrichum. Biology, Pathology and Control (Bailey JA, Jeger MJ eds). CABI, Wallingford, UK: 88-120.

Bancroft. 1910 - West Indian Bulletines 9, 35.

Barimani M, Pethybridge SJ, Vaghefi N, Hay FS et al. 2013 - A new anthracnose disease of pyrethrum by Colletotrichum tanaceti sp. nov. Plant Pathology 62, 1248-1257.

Baroncelli R, Amby DB, Zapparata A, Sarrocco S et al. 2016 - Gene family expansions and contractions are associated with host range in plant pathogens of the genus Colletotrichum. BMC genomics 17, 555.

Baroncelli R, Sanz-Martín JM, Rech GE, Sukno SA, Thon MR. 2014a - Draft genome sequence of Colletotrichum sublineola, a destructive pathogen of cultivated sorghum. Genome Announcements 2, e00540-14.

Baroncelli R, Sarrocco S, Zapparata A, Tavarini S et al. 2015a - Characterization and epidemiology of Colletotrichum acutatum sensu lato (C. chrysanthemi) causing Carthamus tinctorius anthracnose. Plant Pathology 64, 375-384. 
Baroncelli R, Sreenivasaprasad S, Sukno SA, Thon MR, Holub E. 2014b - Draft genome sequence of Colletotrichum acutatum sensu lato (Colletotrichum fioriniae). Genome Announcements 2, e00112-e00114.

Baroncelli R, SuknoSA, Sarrocco S, Cafá G et al. 2018 - Whole-genome sequence of the orchid anthracnose pathogen Colletotruchum orchidophilum. Molecular plant-microbe Interactions 31, 979-981.

Baroncelli R, Zapparata A, Sarrocco S, Sukno SA et al. 2015b - Molecular diversity of anthracnose pathogen populations associated with UK strawberry production suggests multiple introductions of three different Colletotrichum species. PLoS One 10, 129-140.

Batista AC. 1952 - Um pugilo de novos fungos imperfeitos. Boletim da Secretaria de Agricultura Indústria e Comércio do Estado de Pernambuco. 19, 143-150.

Baxter AP, Westhuizen GCA van der, Eicker A. 1983 - Morphology and taxonomy of South African isolates of Colletotrichum. South African Journal of Botany 2, 259-289.

Berkeley MJ. 1851 - Unnamed paper. Gardeners'Chronicle, September 20, 595.

Bhunjun CS, Jayawardena RS, Wei DP, Huanraluek N et al. 2019 - Multigene phylogenetic characterisation of Colletotrichum artocarpicola sp. nov. from Artocarpus heterophyllus in northern Thailand. Phytotaxa 418, 273-286.

Bhunjun CS, Phukhamsakda C, Jayawardena RS, Jeewon R et al. 2021 - Investigating species boundaries in Colletotrichum. Fungal Diversity 107, 107-127.

Bi Y, Guo W, Zhang GJ, Liu SC et al. 2017 - First report of Colletotrichum truncatum causing anthracnose of strawberry in China. Plant Disease 101, 832.

Bisby GR, Buller AHR, Dearness J. 1929 - The fungi of Manitoba 1-194.

Bondartseva-Monteverde VN, Gutner LS, Novoselova ED. 1936 - The parasitic fungi in the greenhouse of the Botanical Institute of the Academy of Sciences of the USSR. Trudy Botanicheskogo Instituta Akademii Nauk SSSR 2. 3, 715-801.

Bondartzeva-Monteverde VN. 1938 - Colletotrichum damasonii. Notul. Syst. Sec. Cryptog. Ins. Bot. Acd. Sci. USSR. 4, 43.

Boufleur TR, Castro RRL, Rogerio F, Ciampi-Guillardi M et al. 2020 - First report of Colletotrichum musicola causing soybean anthracnose in Brazil. Plant Disease 104, 1858.

Bourget R, Chaumont L, Sapoukhina N. 2013 - Timing of pathogen adaptation to a multicomponent treatment. PLoS One 8, e71926.

Braganca CAD, Damm U, Baroncelli R, Massola Jr NS et al. 2016 - Species of the Colletotrichum acutatum complex associated with anthracnose diseases of fruit in Brazil. Fungal Biology 120, 547-561.

Braithwaite KS, Irwin JAG, Manners JM. 1990a - Ribosomal DNA as a molecular taxonomic marker for the group species Colletotrichum gloeosporioides. Australian Systematic Botany 3, 733-738.

Braithwaite KS, Irwin JAG, Manners JM. 1990b - Restriction fragment length polymorphisms in Colletotrichum gloeosporioides infecting Stylosanthes spp. in Australia. Mycological Research 94, 1129-1137.

Braithwaite KS, Manners JM. 1989 - Human hypervariable minisatellite probes detect DNA polymorphisms in the fungus Colletotrichum gloeosporioides. Current Genetics 16, 473-475.

Bresadola G. 1892 - Fungi Tridentini 2, 1-46.

Bresadola G. 1894 - Fungi aliquot saxonici novi vel critici a cl. W. Krieger lecti. (Contributio III ad Floram Mycol. Saxoniae). Hedwigia 33, 206-210.

Briant AK, Martyn EB. 1929 - Diseases of cover crops. Tropical Agriculture 6, 258-260.

Brown CJ, Garner EC, Keith Dunker A, Joyce P. 2001 - The power to detect recombination using the coalescent. Molecular Biology and Evolution 18, 1421-142.

Bruen TC, Philippe H, Bryant D. 2006 - A simple and robust statistical test for detecting the presence of recombination. Genetics 172, 2665-2681.

Bubák F, González FR. 1915 - Fungi nonnulli novi Hispanici. Hedwigia 57, 1-13. 
Busby PE, Ridout M, Newcombe G. 2016 - Fungal Endophytes: modifiers of plant disease. Plant Molecular Biology 90, 645-655.

Butler EJ, Bisby GR. 1931 - The fungi of India. Imperial Council of Agricultural Research Science Monogr 1, 237.

Buyck B, Duhem B, Das K, Jayawarden RS et al. 2017 - Fungal Biodiversity Profiles 21-30. Cryptogamie Mycologie 38, 101-146.

Cabral A, Azinheira HG, Talhinhas P, Batista D. 2020 - Pathological, morphological, cytogenomic, biochemical and molecular data support the distinction between Colletotrichum cigarro comb. et stat. nov. and Colletotrichum kahawae. Plants 9, 502.

Cai ZY, Yang Y, Liu YX, Zhang YM et al. 2016 - First report of Colletotrichum chlorophyti causing Moringa oleifera anthracnose in China. Plant Disease 100, 2164.

Caires NP, Pinho DB, Souza JSC, Silva MA et al. 2014 - First report of anthracnose on pepper fruit caused by Colletotrichum scovillei in Brazil. Plant Disease 98, 1437.

Cannon PF, Bridge PD, Monte E. 2000 - Linking the past, present, and future of Colletotrichum systematics. In: Colletotrichum: Host specificity, Pathology, and Host-pathogen interaction. (Prusky D, Freeman S, Dickman, M, eds). APS Press, St Paul, USA: 1-20.

Cannon PF, Buddie AG, Bridge PD. 2008 - The typification of Colletotrichum gloeosporioides. Mycotaxon 104, 189-204.

Cannon PF, Damm U, Johnston PR, Weir BS. 2012 - Colletotrichum current status and future directions. Studies in Mycology 73, 181-213.

Cao X, Xu X, Che H, West JS et al. 2019 - Three Colletotrichum species, including a new species, are associated to leaf anthracnose of rubber tree in Hainan, China. Plant Disease 103, 117124.

Cao X, Xu X, Che H, West JS et al. 2020 - Eight Colletotrichum species, including a novel species, are associated with areca palm anthracnose in Hainan, China. Plant Disease 104, 1369-1377.

Carbú M, Moraga J, Cantoral JM, Collado IG, Garrido C. 2019 - Recent approaches on the genomic analysis of the phytopathogenic fungus Colletotrichum spp. Phytochemistry Reviews $1-13$.

Carraro TA, Lichtemberg PSF, Michailides TJ, Pereira WV et al. 2019 - First report of Colletotrichum fructicola, C. nymphaeae and C. melonis causing Persimmon anthracnose in Brazil. Plant Disease 103, 2692.

Cavalcante GR, Barguil BM, Vieira WA, Lima WG et al. 2019 - Diversity, prevalence, and virulence of Colletotrichum species associated with Lima Bean in Brazil. Plant Disease 103, 1961-1966.

Cavara F. 1889a - I Funghi Parassitidelle Piante Coltivateed Utili Essicati, Delineati e Descriti, 21.

Cavara F. 1889b. Matériaux de mycologie lombarde. Revue Mycologique Toulouse. 11, 173-193.

Charron C, Hubert J, Minatchy J, Wilson V et al. 2018 - Characterization of Colletotrichum orchidophilum, the agent of black spot disease of vanilla. Journal Phytopathology 166, 525531.

Chaudhuri H. 1924 - A description of Colletotrichum biologicum, nov. sp., and observations on the occurrence of a saltation in the species. Annals of Botany 38, 735-744.

Chechi A, Stahlecker J, Dowling ME, Schnabel G. 2019 - Diversity in species composition and fungicide resistence profiles in Colletotrichum isolates from apples. Pesticide Biochemistry and Physiology 158, 18-24.

Chen CC. 1967 - Survey of epidemic diseases of forest trees in Taiwan III. Botanical Bulletin of the Academia Sinica (Taipei). 8, 130-148.

Chen CC. 1968a - Plant Protection Bulletin 10, 16.

Chen CC. 1968b - Plant Protection Bulletin 10, 17.

Chen F, Goodwin PH, Khan A, Hsiang T. 2002 - Population structure and matingtype genes of Colletotrichum graminicola from Agrostis palustris. Canadian Journal of Microbiology 48, 427-436. 
Chen SY, Hou XM, Yang S, Tan LL. 2019a - First report of Colletotrichum liriopes causing leaf spots on Liriope spicata in China. Plant Disease 103, 1422-1423.

Cheon W, Jeon Y. 2016 - First report of anthracnose caused by Colletotrichum spaethianum on fragrant plantain lily in Korea. Plant Disease 100, 1498-1499.

Chen X, Wang T, Guo H, Zhu PK et al. 2019b - First report of anthracnose of Camellia sasanqua caused by Colletotrichum aenigma in China. Plant Disease 103, 1423.

Chester FD. 1894 - Report of mycologist. Report of the Delaware Agricultural Experimental Station 6.

Chethana CS, Chowdappa P, Biju CN, Praveena R et al. 2016 - Molecular and phenotypic characterization revealed six Colletotrichum species responsible for anthracnose disease of small cardamom in South India. European Journal of Plant Pathology 146, 465-481.

Chethana KWT, Jayawardene RS, Zhang W, Zhou YY et al. 2019 - Molecular characterization and pathogenicity of fungal taxa associated with cherry leaf spot disease. Mycosphere 10, 490530.

Chevassut G, Pellicier P. 2001 - Huitespècesnouvelles de micromycetes (Fungi imperfecti) parasites des plantesspontanées, récoltesen France. Bulletin de la Société Mycologique de France. 117, 193-206.

Chevaugeon 1952 - Riv. Patol. veg. 31, 36.

Chiplonkar A. 1969 - New phytopathogenic Fungi Imperfecti from Maharashtra (India). Sydowia 23, 106-109.

Choi HW, Lee YK, Hong SK. 2017 - First report of Colletotrichum aenigma causing anthracnose on Sedum kamtschaticumin Korea. Plant Disease 101, 2150.

Chowdappa P, Chethana CS, Pant RP, Bridge PD. 2014 - Multilocus gene phylogeny reveals occurrence of Colletotrichum cymbidiicola and C. Cliviae on orchids in North East India. Journal of Plant Pathology 96, 327-334.

Cong YL, Hou J, Liu LP, Liu ZY et al. 2020 - First report of anthracnose disease caused by Colletotrichum truncatum on the velvetleaf (Abutilon theophrasti) in China. Plant Disease $104,565$.

Corda ACI. 1831 - Die Pilze Deutschlands. In: Sturm J (ed.) Deutschlands Flora in Abbildungen nach der Natur mit Beschreibungen. Sturm, Nürnberg vol. 3, Abt. 12, 33-64, tab, 21-32.

Corda ACJ. 1837 - Icones fungorum hucusque cognitorum. 1, 1-32.

Corda ACJ. 1840 - Icones fungorum hucusque cognitorum. 4, 1-53.

Costa JFO, Kamei SH, Silva JRA, Miranda ARGS. 2019 - Species diversity of Colletotrichum infecting Annona spp. in Brazil. European Journal of Plant Pathology 153, 1119-1130.

Costa ME, Souza da Camara M de. 1953 - Species aliquae mycologicae Lusitaniae. II. Portugaliae Acta Biologica 4, 162-176.

Cristobal-Martinez AL, de Jesus Yanez-Morales M, Solano-Vidal R, Segura-Leon O et al. 2017 Diversity of Colletotrichum species in coffee (Coffea arabica) plantations in Mexico. European Journal of Plant Pathology 147, 605-614.

Crouch JA, Clarke BB, Hillman BI. 2006 - Unraveling evolutionary relationships among the divergent lineages of Colletotrichum causing anthracnose disease in turfgrass and maize. Phytopathology 96, 46-60.

Crouch JA, Glasheen BM, Giunta MA, Clarke BB, Hillman BI. 2008 - The evolution of transposon repeat-induced point mutation in the genome of Colletotrichum cereale: reconciling sex, recombination and homoplasy in an 'asexual'pathogen. Fungal Genetics and Biology 45, 190-206.

Crouch JA, Tomaso-Peterson M. 2012 - Anthracnose disease of centipedegrass turf caused by Colletotrichum eremochloae, a new fungal species closely related to Colletotrichum sublineola. Mycologia 104, 1085-1096.

Crouch JA, Clarke BB, White JF Jr, Hillman BI. 2009a - Systematic analysis of the falcate-spored graminicolous Colletotrichum and a description of six new species from warm-season grasses. Mycologia 101, 717-732. 
Crouch JA, Tredway LP, Clarke BB, Hillman BI. 2009b - Phylogenetic and population genetic divergence correspond with habitat for the pathogen Colletotrichum cereale and allied taxa across diverse grass communities. Molecular Ecology 18, 123-135.

Crouch JA, Beirn LA, Cortese LM, Bonos SA et al. 2009c - Anthracnose disease of switch grass caused by the novel fungal species Colletotrichum navitas. Mycological Research 113, 14111421.

Crouch JA, O’Connell R, Gan P, Buiate E et al. 2014 - The genomics of Colletotrichum. Genomics of plant-associated fungi, monocot pathogens, Springer, Berlin, Heidelberg, pp. 69-102.

Crous PW, Luangsa-Ard JJ, Wingfield MJ, Carnegie AJ et al. 2018a - Fungal Planet description sheets, 785-867. Persoonia 41, 238.

Crous PW, Wingfield MJ, Burgess TI, St J Hardy GE et al. 2018b - Fungal planet description sheets, 716-784. Persoonia 40, 240-393.

Crous PW, Wingfield MJ, Guarro J, Cheewangkoon R et al. 2013 - Fungal Planet description sheets, 154-213. Persoonia 31, 188-296.

Crous PW, Wingfield MJ, Guarro J, Hernandez-Restrepo M et al. 2015 - Fungal Planet description sheets, 320-370. Persoonia 34, 167-266.

Crous PW, Wingfield MJ, Richardson DM, Le Roux JJ et al. 2016 - Fungal Planet description sheets, 400-468. Persoonia 36, 316-458.

Crous PW, Carnegie Aj, Wingfield MJ, Sharma R et al. 2019 - Fungal Planet description sheets, 868-950. Persoonia 42, 291-473.

Curry KJ, Abril M, Avant JB, Smith BJ. 2002 - Strawberry anthracnose, Histopathology of Colletotrichum acutatum and C. fragariae. Phytopathology 92, 1055-1063.

Da-Lio D, Cobo-Díaz JF, Masson C, Chalopin M et al. 2018 - Combined metabarcoding and multilocus approach for genetic characterization of Colletotrichum species associated with common walnut (Juglans regia) anthracnose in France. Scientific Reports 8, 1-17.

Dale JL, Manners JM, Irwin JAG. 1988 - Colletotrichum gloeosporioides isolates causing different anthracnose diseases on Stylosanthes in Australia carry distinct double-stranded RNAs. Transactions of the British Mycological Society 91, 671-676.

Damm U, Woudenberg JHC, Cannon PF, Crous PW. 2009 - Colletotrichum species with curved conidia from herbaceous hosts. Fungal Diversity 39, 45-87.

Damm U, Barroncelli R, Cai L, Kubo Y et al. 2010 - Colletotrichum: species, ecology and interactions. IMA Fungus 1, 161-165.

Damm U, Cannon PF, Woudenberg JHC, Johnston PR et al. 2012a - The Colletotrichum boninense species complex. Studies in Mycology 73, 1-36.

Damm U, Cannon PF, Woudenberg JHC, Crous PW. 2012b - The Colletotrichum acutatum species complex. Studies in Mycology 73, 37-113.

Damm U, Cannon PF, Liu F, Barreto RW et al. 2013 - The Colletotrichum orbiculare species complex: important pathogens of field and weeds. Fungal Diversity 61, 29-59.

Damm U, O’Connell RJ, Groenewald JZ, Crous PW. 2014 - The Colletotrichum destructivum species complex - hemibiotrophic pathogens of forage and field crops. Studies in Mycology 79, 49-84.

Damm U, Sato T, Alizadeh A, Groenewald JZ, Crous PW. 2019 - The Colletotrichum dracaenophilum, C. magnum and $C$. orchidearum species complexes. Studies in mycology 92, 1-46.

Damm U, Sun YC, Huang CJ. 2020 - Colletotrichum eriobotryae sp. nov. and C. nymphaeae, the anthracnose pathogens of loquat fruit in Central Thaiwan, and their sensitivity to azoxystrobin. Mycological Progress 19, 367-380.

da Silva LL, Moreno HLA, Correia HLN, Santana MF, de Queiroz MV. 2020 - Colletotrichum: Species complexes, lifestyle, and peculiarities of some sources of genetic variability. Applied Microbiology and Biotechnology 104, 1891-1904.

Dastur. 1934 - Indian Journal of Agricultural Research 4, 118. 
Davis JI, Nixon KC. 1992 - Populations, genetic variation, and the delimitation of phylogenetic species. Systematic Biology 41, 421-35.

Davis JJ. 1915 - Notes on parasitic fungi in Wisconsin III. Transactions of the Wisconsin Academy of Science. 18, 251-271.

Davis JJ. 1919 - Notes on parasitic fungi in Wisconsin. IV. Transactions of the Wisconsin Academy of Science. 19, 671-689.

Davis JJ. 1929 - Notes on parasitic fungi in Wisconsin XVII. Transactions of the Wisconsin Academy of Science. 24, 295-301.

de Silva DD, Ades PK, Crous PW, Taylor PWJ. 2017 - Colletotrichum species associated with chili anthracnose in Australia. Plant Pathology 66, 254-267.

de Silva DD, Groenewald JZ, Crous PW, Ades PK et al. 2019 - Identification, prevalence and pathogenicity of Colletotrichum species causing anthracnose of Capsicum annum in Asia. IMA Fungus 10, 8.

de Silva NI, Maharachchikumbura SSN, Thambugala KM, Bhat DJ et al. 2021 - Morphomolecular taxonomic studies reveal a high number of endophytic fungi from Magnolia candolli and $M$. garrettii in China and Thailand. Mycosphere 11, 163-237.

de Souza ESC, do Vale HMM, Carvalho RCP, Soares WRO et al. 2017 - Infection by Uromyces euphorbiae, a trigger for the sporulation of the endophytic Colletotrichum truncatum on the common host Euphorbia hirta. Mycological Progress 16, 941-946.

Dearness J. 1928 - New and noteworthy fungi. V. Mycologia. 20, 235-246.

Degen 1938 - Dokl. Akad. Nauk SSSR 3, 295.

Delacroix G. 1905 - Travaux de la Station de Pathologievégétale. Bulletin de la Société Mycologique de France. 21, 168-204.

Delaye L, García-Guzmán G, Heli M. 2013 - Endophytes versus biotrophic and necrotrophic pathogens-are fungal lifestyles evolutionary stable traits? Funagl Diversity 60, 125-135.

Dettman JR, Jacobson DJ, Taylor JW. 2003 - A multilocus genealogical approach to phylogenetic speciesrecognition in the model eukaryote Neurospora. Evolution 57, 2703-2720.

Diao YZ, Zhang C, Liu F, Wang WZ et al. 2017 - Colletotrichum species causing anthracnose disease of chili in China. Persoonia 38, 20-37.

Dias LRC, Brito RAS, Melo TA, Serra IMRS. 2020 - First report of papaya fruit anthracnose caused by Colletotrichum okinawense in Brazil. Plant Disease 104, 573.

Dias MRS, Sousa da Câmara M. 1952 - Fungi Lusitaniae. I. AgronomiaLusitana. 14, 101-125.

Dias MRS, Sousa da Câmara M. 1953 - Fungi Lusitaniae. IV. AgronomiaLusitana. 15, 17-37.

Dickson BT. 1926 - The 'black dot' disease of the potato. Phytopathology 16, 23-40.

Dickson BT. 1925 - Colletotrichum v. Vermicularia. Mycologia. 17, 213-217.

Diedicke H. 1915 - Pilze, Sphaeropsideae, Melanconieae. Kryptogamen-Flora der Mark Brandenburg. 9, 801-962.

Dobrozrakova. 1927 - Morbi plant. Leningrad 16, 202.

Douanla-Meli C, Unger JG, Langer E. 2018 - Multi-approach analysis of the diversity in Colletotrichum cliviaesensu lato. Antonie van Leeuwenhoek 111, 423-435.

Doyle VP, Oudemans PV, Rehner SA, Litt A. 2013 - Habitat and host indicate lineage identity in Colletotrichum gloeosporioidess.l. from wild and agricultural landscapes in North America. PLoS One 8, 62394.

Duke MM. 1928 - The genera Vermicularia Fr. and Colletotrichum Cda. Transactions of the British Mycological Society 13, 156-184.

Dubrulle G, Pensec F, Picot A, Rigalma K et al. 2020 - Phylogenetic diversity and effect of temperature on pathogenicity of Colletotrichum lupini. Plant Disease 104, 938-950.

Du M, Schardl CL, Nuckles EM, Vaillancourt LJ. 2005 - Using mating-type gene sequences for improved phylogenetic resolution of Colletotrichum species complexes. Mycologia 97, 641658.

Dzhalagoniya. 1962 - Novit. Mycol. Nov. 14, 63. 
Dzhalagoniya. 1965 - Parazitnye griby glavneishikh subtropicheskikh dekorativnykh rastenii Abkhazii [Parasitic fungi of the main subtropical decorative plants of Abkhazia] Tiflis, 46.

Earle FS. 1897 - New species of fungi imperfecti from Alabama. Bulletin of the Torrey Botanical Club. 24, 28-32.

Earle FS. 1902 - Mycological studies. I. Bulletin of the New York Botanical Garden. 2, 331-350.

Eaton MJ, Edwards S, Inocencio HA, Machado FJ et al. 2011 - Diversity and cross-infection potential of Colletotrichum causing fruit rots in mixed fruit orchards in Kentucky. Plant Disease PDIS-06.

Echeverrigaray S, Scariot FJ, Fontanella G, Favaron F et al. 2020 - Colletotrichum species causing grape ripe rot disease in Vitis labrusca and $V$. vinifera varieties in the highlands of southern Brazil. Plant Pathology 69, 1504-1512.

Edgerton 1908 - Anthacnoses. Botanical Gazette. 45, 408.

Elisei FG. 1938 - Nuovi deuteromiceti parassiti foglicoli di dicotiledoni. Atti dell'Istituto Botanico della Università e Laboratorio Crittogamico di Pavia. 10, 233-240.

Ellis JB, Everhart BM. 1895 - New species of fungi. I. Sandwich Island fungi. II. Florida fungi. III. Mexican fungi. Bulletin of the Torrey Botanical Club 22, 434-440.

Ellis JB, Everhart BM. 1897 - New species of fungi from various localities. Bulletin of the Torrey Botanical Club 24, 457-477.

Ellis JB, Everhart BM. 1902 - New Alabama fungi. Journal of Mycology 8, 62-73.

Fabricatore 1950, Atti Imp. Regia Accad. Rovereto, ser. 3, 6, 139

Farr DF, Aime MC, Rossman AY, Palm ME. 2006 - Species of Colletotrichum on agavaceae. Mycological Research 110, 1395-1408.

Farr DF, Rossman AY. 2021 - Fungal Databases, Systematic Mycology and Microbiology Laboratory, ARS, USDA. http://nt.ars-grin.gov/fungaldatabases/

Farr ML. 1960 - Mycological notes. New species, combinations, and host records. Mycopathologia et Mycologia Applicata 12, 283-288.

Fealko E, Szarka D, Lamb A, Amsden B et al. 2019 - First report of black dot root rot, caused by Colletotrichum coccodes, on tomato in Kentucky high tunnels. Plant Disease 103, 21262127.

Forseille L, Peng G, Gossen BD, Wei YD. 2009 - Further evidence for host specificity of Colletotrichum truncatum from scentless chamomile. Canadian Journal of Plant Pathology 31, 301-308.

Frantz LA, Schraiber JG, Madsen O, Megens HJ et al. 2013 - Genome sequencing reveals fine scale diversification and reticulation history during speciation in Sus. Genome Biology 14, 112.

Freeman S, Minz D, Maymon M, Zveibil A. 2001 - Genetic diversity within Colletotrichum acutatum sensu Simmonds. Phytopathology 91, 586-592.

Freeman S, Rodriguez RJ. 1993 - Genetic conversion of a fungal plant pathogen to a nonpathogenic endophytic mutualist. Science 260, 75-78.

Fu M, Crous PW, Bai Q, Zhang PF et al. 2019 - Colletotrichum species associated with anthracnose of Pyrus spp. in China. Persoonia 42, 1-35.

Fuentes-Aragon D, Silva-Rojas HV, Guarnaccia V, Mora-Aguilera JA et al. 2020 - Colletotrichum species causing anthracnose on avocado fruit in Mexico, current status. Plant Pathology 69, $1513-1528$.

Fujita MK, Leache AD. 2011 - A coalescent perspective on delimiting and naming species, a reply to Baueret al. Proceedings of the Royal Society B, Biological Sciences 278,493-495.

Fujita MK, Leache AD, Burbrink FT, McGuire JA, Mortiz C. 2012 - Coalescent-based species delimitation in an integrative taxonomy. Trends in Ecology and Evolution 27, 480-488.

Fukui T. 1925 - Journal of Plant Protection 12.

Gabotti. 1914 - Bol. Soc. Bot. Ital., 103.

Gallucci-Rangone. 1955 - Annali Sper. agr. 9 (3, Suppl.), 14. 
Gan P, Ikeda K, Irieda H, Narusaka M et al. 2013 - Comparative genomic and transcriptomic analyses reveal the hemibiotrophic stage shift of Colletotrichum fungi. New Phytologist 197, 1236-1249.

Gan P, Narusaka M, Kumakura N, Tsushima A et al. 2016 - Genus-wide comparative genome analyses of Colletotrichum species reveal specific gene family losses and gains during adaptation to specific infection lifestyles. Genome Biology and Evolution 8, 1467-1481.

Gan P, Narusaka M, Tsushima A, Narusaka Y et al. 2017 - Draft genome assembly of Colletotrichum chlorophyti, a pathogen of herbaceous plants. Genome Announcements 5, 01733-16 PMID, 28280027.

Gan P, Tsushima A, Narusaka M, Narusaka Y et al. 2019 - Genome sequence resources for four phytopathogenic fungi from the Colletotrichum orbiculare species complex. Molecular PlantMicrobe Interactions 32, 1088-1090.

Gazis R, Rehner S, Chaverri P. 2011 - Species delimitation in fungal endophyte diversity studies and its implications in ecological and biogeographic inferences. Molecular Ecology 20, 3001-3013.

Gatesy J, Baker RH. 2005 - Hidden likelihood support in genomic data, can forty-five wrongs make a right?. Systematic Biology 54, 483-492.

Gautam AK, Avasthi S, Bhadauria R. 2012 - Colletotrichum sansevieriae on Sansevieria trifasciata- a report from Madhya Pradesh, India. Plant Pathology \& Quarantine 2, 190-192.

Goldstein PZ, Desalle R, Amato G, Vogler AP. 2000 - Conservation genetics at the species boundary. Conservation Biology 14, 120-31.

Golzar H. 2009 - First report of anthracnose of Acacia in Australia. Australasian Plant Disease Notes 4, 70-71.

Golzar H, Wang C. 2010 - First report of Colletotrichum phormii the cause of anthracnose on Phormium tenax in Australia. Australasian Plant Disease Notes 5, 110-112.

González FR. 1924 - Contribución a la flora micológica lusitánica. Boletim da Sociedade Broteriana. 2, 3-83.

González FR, Ciferri R. 1925 - Hongos parásitos y saprofitos de la República Dominicana (1a serie). Boletín de la Real Sociedad Española de Historia Natural 25, 356-368.

González FR, Ciferri R. 1927 - Boln de la Real Soc. Españ. Hist. Nat., Madrid 27, 330.

Goodman RN. 1960 - Observation on the production, physiological activity and chemical nature of Colletotin, a toxin from Colletotrichum fuscum Laub. Journal of Phytopathology 37, 187194.

Goodwin PH. 2001 - A molecular weed-mycoherbicide interaction: Colletotrichum gloeosporioides f. sp. malvae and round-leaved mallow, Malva pusilla. Canadian Jouranl of Plant Pathology 23, 28-35.

Grammen A, Wenneker M, Van Campenhout J, Pham KTK et al. 2019 - Identification and pathogenicity assessment of Colletotrichum isolates causing bitter rot of apple fruit in Belgium. European Journal of Plant Pathology 153, 47-63.

Greene HC. 1950 - Notes on Wisconsin parasitic fungi. XIV. The American Midland Naturalist. $44,630-642$.

Greene HC. 1951 - In, Am. Midl. Nat. 44, 640.

Greene HC. 1953a - Notes on Wisconsin parasitic fungi. XIX. The American Midland Naturalist. 50, 501-508.

Greene HC. 1953b - Notes on Wisconsin parasitic fungi. XVIII. Transactions of the Wisconsin Academy of Science. 42, 69-81.

Greene HC. 1955 - Notes on Wisconsin parasitic fungi. XXI. Transactions of the Wisconsin Academy of Science. 44, 29-43.

Greene HC. 1956 - Notes on Wisconsin parasitic fungi. XXII. Transactions of the Wisconsin Academy of Science. 45, 177-191.

Griffon MM, Maublanc 1909 - Notes de Mycologie et de Pathologie végétale. Bulletin trimestriel de la Société mycologique de France. 25, 59. 
Grigoriev IV, Cullen D, Goodwin SB, Hibbett D et al. 2011 - Fueling the future with fungal genomics. Mycology 2, 192-209.

Grove WB. 1918 - New or noteworthy fungi. VI [part 3]. Journal of Botany, British and Foreign. $56,340-346$.

Grove WB. 1919 - Species placed by Saccardo in the genus Phoma. Part 1. Bulletin of Miscellaneous Informations of the Royal Botanical Gardens Kew 1919, 177-201.

Guan YM, Liu ZB, Li MJ, Wang QX et al. 2018 - First report of Colletotrichum spaethianum causing anthracnose in Atractylodes japonica in China. Plant Disease 102, 239.

Guarnaccia V, Gilardi G, Martino I, Garibaldi A et al. 2019 - Species diversity in Colletotrichum causing anthracnose of aromatic and ornamental Lamiaceae in Italy. Agronomy 9, 613.

Guarnaccia V, Groenewald JZ, Polizzi G, Crous PW. 2017 - High species diversity in Colletotrichum associated with citrus diseases in Europe. Persoonia 39, 32-50.

Guerber JC, Liu B, Correll JC, Johnston PR. 2003 - Characterization of diversity in Colletotrichum acutatum sensu lato by sequence analysis of two gene introns, mtDNA and intron RFLPs, and mating compatibility. Mycologia 95, 872-895.

Guo M, Pan YM, Dai YL, Gao ZM. 2013 - First report of leaf spot caused by Colletotrichum spaethianum on Peucedanum praeruptorum in China. Plant Disease 97, 1380.

Gupta PC. 1974a - Z. PflKrankh. PflPath. PflSchutz 81, 90.

Gupta PC. 1974b - Z. PflKrankh. PflPath. PflSchutz 81, 91.

Gurzitska 1950 - Parasitnie Nesov. Grib. 2, 304.

Gutner LS. 1933a - Gribi-parasitioranñereynikhrastenii Leningradai Detskogo Selo. Trudy Botanicheskogo Instituta Akademii Nauk SSSR 2. 1, 285-323.

Gutner LS. 1933b - Diseases pests of new cultivated textile plants (Inst. New Bast Raw Material Vaskhnil, Moscow), 71-72.

Hacquard S, Kracher B, Hiruma K, Münch PC et al. 2016 - Survival trade-offs in plant roots during colonization by closely related beneficial and pathogenic fungi. Nature Communications 7, 1-13.

Hall CJJ, Drost 1908 - Recueil des Travaux botaniques Neerlandais 4, 243.

Halsted BD. 1891 - Report of the New Jersey State Agricultural Experimental Station, 293.

Halsted BD. 1893a - Some new weed fungi. Bulletin of the Torrey Botanical Club 20, 250-252.

Halsted BD. 1893b - Identity of anthracnose of the bean and watermelon. Bulletin of the Torrey Botanical Club 20, 246-250.

Han S, Jiang Y, Yi J, Li S et al. 2020 - First report of Colletotrichum fioriniae infection on Zanthoxylum armatumin China. Plant Disease 104, 2292.

Han YZ, Fan ZW, Wu CF, Wang JH et al. 2019 - First report of leaf anthracnose caused by Colletotrichum cliviicola on hybrid Pennisetum in China. Plant Disease 103, 2472.

Han YC, Zeng XG, Xiang FY, Ren L et al. 2016 - Distribution and characteristics of Colletotrichum spp. Associated with anthracnose of strawberry in Hubei China. Plant Disease 100, 996-1006.

Hara K.1930 - Rept. Siduokaken Agric. Soc. 34, 49-52.

Hara K. 1954 - A list of Japanese fungi hitherto known. 1-447.

Hara K. 1959 - A Monograph of Rice Diseases.

Hardoim PR, Van Overbeek LS, Berg G, Pirttilä AM et al. 2015 - The hidden world within plants: ecological and evolutionary considerations for defining functioning of microbial endophytes. Microbiology and Molecular Biology Reviews 79, 293-320.

Hasija SK. 1962a - Proc. natn. Acad. Sci. India 32, 413.

Hasija SK. 1962b - A new species of Colletotrichum. Current Science 31, 107.

Hasija SK. 1962c - Additions to the fungi of Jabalpur. (Madhya Pradesh) - I. Mycopathologia et Mycologia Applicata. 18, 84-92.

Hassan O, Lee DW, Chang T. 2019 - First report of anthracnose of persimmon caused by Colletotrichum nymphaeae in Korea. Plant Disease 103, 1172.

Heald FD, Wolf FA. 1911 - New species of Texas fungi. Mycologia. 3, 5-22. 
Hemmi. 1920 - Journal of the Sapporo agric. Coll. 9, 22.

Henk DA, Eagle CE, Brown K, Van Den Berg MA et al. 2011 - Speciation despite globally overlapping distributions in Penicillium chrysogenum, the population genetics of Alexander Fleming'slucky fungus. Molecular Ecology 20, 4288-4301.

Hennings P. 1908a - Fungi S. Paulensis IV a cl. Puttemanscollecti. Hedwigia 48, 1-20.

Hennings P .1908b - Fungi paraënses III. Hedwigia. 48, 101-117.

Hennings P. 1904a - Einigeneue Pilzeaus Japan. Hedwigia 43, 140-146.

Hennings P. 1904b - Fungi fluminenses a. cl. E. Ulecollecti. Hedwigia 43, 78-95.

Hennings P. 1905a - Einige Schädliche Parasitische Pilze auf exotischen Orchideenunserer Gewachshauser. Hedwigia 44, 168-178.

Hennings P. 1905b - Fungi Amazonici IV. a cl. Ernesto Ulecollecti. Hedwigia 44, 57-71.

Hennings P. 1906 - Deutsche Südpolar-Exped., Bd. (extr.) 8, 15.

Hino I, Katumoto K. 1958 - Illustrationes fungorum bambusicolorum. VI. Bulletin of the Faculty of Agriculture Yamaguchi University. 9, 877-908.

Hiruma K, Gerlach N, Sacristán S, Nakano RT et al. 2016 - Root endophyte Colletotrichum tofieldiae confers plant fitness benefits that are phosphate status dependent. Cell 165, 464474.

Höhnel F von. 1919 - Fünftevorläufige Mitteilungmykologische Ergebnisse (Nr. 300-500). Berichte der Deutschen Botanischen Gesellschaft 37, 153-161.

Höhnel F von. 1957 - Rev. Derzu Gloeosporium gestellten Pilze, 48

Hollós L. 1908 - Új gombák Kecskemét vidékérol. V. Annales Historico-Natureles Musei Nationalis Hungarici. 6, 527-536.

Hoof 1949 - Annales of phytopathological Society of Japan 13, 8.

Hori 1919 - Special Bulletin Agricultural Experiment Station Formosa 19, 583.

Horn NL. 1952 - A comparative study of two species of Colletotrichum on vetch. Phytopathology $42,670-674$.

Hou LW, Liu F, Duan WJ, Cai L. 2016 - Colletotrichum aracearum and C. camelliae-japonicae, two holomorphic new species from China and Japan. Mycosphere 7, 1111-1123.

Howard RJ, Ferrari MA. 1989 - Role of melanin in appressorium function. Experimental Mycology 13, 403-418.

Hsieh CW, Chuang YY, Lee MZ, Kirschner R. 2020 - First inventory of fungi in symptomless and symptomatic Chinese Mesona indicates phytopathological threat. Plant Disease 104, 23912397.

Huang F, Chen Gq, Hou X, Fu YS et al. 2013 - Colletotrichum species associated with cultivated citrus in China. Fungal Diversity 61, 61-74.

Hudson RR, Coyne JA. 2002 - Mathematical consequences of the genealogical species concept. Evolution 56, 1557-65.

Hughes SJ. 1958 - Revisiones Hyphomycetum aliquot cum appendice de nominibus rejiciendis. Canadian Journal of Botany. 36, 727-836.

Hulea A. 1939 - Acad. Roum. Bull. Sect. Scient. 22, 212.

Hulea A. 1950 - Herb. Mycologicum Romanicum fasc. 30, no. 1480.

Hung N, Hsiao CH, Yang CS, Lin HC et al. 2020 - Colletotrichum keratitis, a rare yet important fungal infection of human eyes. Mycoses 63, 407-415.

Hunupolagama DM, Chandrasekharan NV, Wijesundera WSS, Kathriarachchi HS et al. 2017 Unveiling members of Colletotrichum acutatum species complex causing Colletotrichum leaf disease of Hevea brasiliensis in Sri Lanka. Current Microbiology 74, 747-756.

Hüseyin, Selçuk. 2001 - Lidia 5, 149.

Huson DH, Scornavacca C. 2011 - A survey of combinatorial methods for phylogenetic networks. Genome Biological Evolution 3, 23-35.

Hyde KD, Cai L, McKenzie EHC, Yang YL et al. 2009a - Colletotrichum: a catalogue of confusion. Fungal Diversity 39, 1-17. 
Hyde KD, Cai L, Cannon PF, Crouch JA et al. 2009b - Colletotrichum-names in current use. Fungal Diversity 39, 147-182.

Hyde KD, Nilsson RH, Alias SA, Ariyawansa HA et al. 2014 - One stop shop: backbones trees for important pytopathogenic genera: I. Fungal Diversity 67, 21-125.

Hyde KD, Hongsanan S, Jeewon R, Bhat DJ et al. 2016 - Fungal diversity notes 367-491: taxonomic and phylogenetic contributions to fungal taxa. Fungal Diversity 80, 1-270.

Hyde KD, Norphanphoun C, Abreu VP, Bazzicalupo A et al. 2017 - Fungal Diversity notes 603708, taxonomic and phylogenetic notes on genera and species. Fungal Diversity 87, 1-235.

Hyde KD, Norphanphoun C, Maharachchikumbura SSN, Bhat DJ et al. 2020 - Refined families of Sordariomycetes. Mycosphere 11, 305-1059.

Ikata S, Yoshida M. 1940 - A new anthracnose of jute-plant. Annals of the Phytopathological Society of Japan 10, 141-149.

Istvanfy. 1902 - Mitt. K. Ungar. ampel. Central-Anstalt. 2.

Ito K, Kobayashi T. 1958 - Studies on some anthracnoses of woody plants - V. Anthracnose fungi of black locust. Bulletin of the Government Forest Experimental Station Meguro 108, 1-29.

Jaczewski ALA. 1906 - Notes phytopathologiques - Alternaria grossulariae n. sp. et Colletotrichum grossulariae n. sp. Bulletin de la Société Mycologique de France 22, 121124.

Jayawardena RS, Hyde KD, Damm U, Cai L et al. 2016a - Notes on currently accepted species of Colletotrichum. Mycosphere 7, 1192-1260.

Jayawardena RS, Huang J, Jin B, Yan JY et al. 2016b - Morphology and molecular phylogeny updated Colletotrichum species associated with strawberry anthracnose in China. Mycosphere 7, 1147-1163.

Jaywardena RS, Camporesi E, Elgorban AM, Bahkali AH et al. 2017 - A new species of Colletotrichum from Sonchus sp. in Italy. Phytotaxa 314, 55-63.

Jayawardena RS, Hyde KD, Chethana KWT, Daranagama DA et al. 2018 - Mycosphere notes 102-168, Saprotrophic fungi on Vitis in China, Italy, Russia and Thailand. Mycosphere 9, 1114.

Jayawardena RS, Hyde KD, Chen YJ, Papp V et al. 2020 - One stop shop IV: taxonomic update with molecular phylogeny for important phytopathogenic genera: 76-100 (2020). Fungal Diversity 103, 87-218.

Jensen, Stewart. 1911 - Phytopathology 1, 125.

Johnson DA, Carris LM, Rogers JD. 1997 - Morpological and molecular characterization of Colletotrichum nymphaeae and C. nupharicola sp. nov. on water lilies (Nymphaea and Nuphar). Mycological Research 101, 641-649.

Joly P. 1965 - Flore Du Vietnam. Bulletin de la Société Mycologique de France 81, 269-287.

Kabát F, Bubák JE. 1907 - Mykologische Beiträge IV. Hedwigia 46, 297.

Kamal, Singh RP. 1980 - Indian Phytopath. 33, 594.

Kanto T, Uematsu S, Tsukamoto T, Moriwaki J et al. 2014 - Anthracnose of sweet pepper caused by Colletotrichum scovillei in Japan. Journal of Genetics and Plant Pathology 80, 73-78.

Karakulin. 1924 - Morbi plant. Leningrad 13, 133.

Karamnejadi T, Solhizadeh A, Shenavar O, Tajick-Ghanbari MA. 2017 - First report of leaf spot caused by Colletotrichum coccodes on Viola tricolor in Iran. Journal of Plant Pathology 99, 536.

Karimi O, Tajick Ghanbari MA, Bagherabadi S, Moradi Amirabad Y. 2017 - First report of Colletotrichum sansevieriae causing anthracnose on Sansevieria trifasciata in Iran. Journal of Plant Pathology 99, 302.

Katsura 1951 - Forsch. PflKr., Tokyo 4, 105.

Kelkar 1972 - Maharashtra Vidnyan Mandir, Patrika 7, 47.

Kelkar PV, Rao VG. 1962 - Some new records of Colletotrichum species from Bombay Maharashtra (India). Sydowia 16, 101-105. 
Kellerman WA, Swingle WT. 1888 - New species of Kansas Fungi. Journal of Mycology. 4, 9395.

Khan A, Hsiang T. 2003 - The infection process of Colletotrichum graminicola and relative aggressiveness on four turfgrass species. Canadian Journal of Microbiology 49, 433-442.

Kiers TE, Palmer TM, Ives AR, Bruno JF, Bronstein JL. 2010 - Mutualism in a changing worl: an evolutionary perspective. Ecology Letters 13, 1459-1474.

Kim JH, Jeong US, Cheong SS, Lee KK et al. 2012 - Anthracnose of black raspberry caused by Colletotrichum gloeosporioides, C. coccodes and C. acutatum in Korea. Phytotaxa 18, 62-64.

Kim CO, Hassan O, Lee D, Chang T. 2018 - First report of anthracnose of Apple caused by Colletotrichum fructicola in Korea. Plant Disease 102, 2563.

Klaptzova. 1940 - Notulae Syst. Sect. Crypt. Inst. Bot. Acad. Sci. U.S.S.R. 5, 8.

Koek MM, Jellema RH, van der Greef J, Tas AC, Hankemeier T. 2011 - Quantitative metabolomics based on gas chromatography mass spectrometry: status and perspectives. Metabolomics 7, 307-328.

Koorders SH. 1907 - Botanische Untersuchungen. Verhandelingen Koninklijke Nederlandse Akademie van Wetenschappen Afdeling Natuurkunde. 13, 1-264.

Koorders Z. 1905 - Over enige ziekten en plagen van Ficus elastica. Bulletin van het Proefstation in Salatiga (Java). 3, 6-13.

Kostlan 1905 - Festschr. 70. Geburtstage A. Orth., 113.

Koval 1961 - Journal of Botanical Academy of Science Ukraine 18, 79.

Kubatko LS, Gibbs HL, Bloomquist EW. 2011 - Inferring species-level phylogenies and taxonomic distinctiveness using multilocus data in Sistrurus rattlesnakes. Systematic Biology 60, 393-409.

Kuhnholtz-Lordat G, Barry JP. 1949 - Quelques micromycètes parasites intéressants du sud de la France. Bulletin de la Société Mycologique de France 65, 121-131.

Kurlovich BS. 2002 - (ed.) Lupins: geography, classification, genetic resources and breeding. Bougouslav Kourlovitch.

Kwashnina. 1928 - Mitteil. Nord-Kaukas. Stat. Pflanzenschutz 4.

Lal. 1953 - Journal of Indian botanical Society 32, 88.

Laluk K, Mengiste T. 2010 - Necrotroph attacks on plants, wanton destruction or covert extortion?. The Arabidopsis Book/American Society of Plant Biologists, $\mathrm{p} 8$.

Latunde-Dada AO. 2001 - Colletotrichum: tales of forcible entry, stealth, transient confinement and breakout. Molecular Plant Pathology 2, 187-198.

Latunde-Dada AO, O'Connell RJ, Nash C, Pring RJ et al. 1996 - Infection process and identity of the hemibiotrophic anthracnose fungus (Colletotrichum destructivum) from cowpea (Vigna unguiculata) Mycological Research 100, 1133-1141.

Leavitt SD, Johnson LA, Goward T, St Clair LL. 2011 - Species delimitation in taxonomically difficult lichen-forming fungi, an example from morphologically and chemically diverse Xanthoparmelia (Parmeliaceae) in North America. Molecular Phylogenetics and Evolution 60, 317-332.

Lee D, Hassan O, Kim C, Chang T. 2018 - First report of peach (Prunus persica) anthracnose caused by (Colletotrichum fioriniae) in Korea. Plant Disease 102, 2650-2651.

Lee DM, Hassan O, Chang T. 2020 - Identification, characterization, and pathogenicity of Colletotrichum species causing anthracnose of peach in Korea. Mycobiology 48, 210-218.

Lee SY, Jung HY. 2018 - Colletotrichum kakivorum sp. nov, a new leafspot pathogen of persimmon in Korea. Mycological Progress 17, 1113-1121.

Leite B, Nicholson RL. 1992 - Mycosporine-alanine: A self-inhibitor of germination from the conidial mucilage of Colletotrichum graminicola. Experimental Mycology 16, 76-86.

Lelwala RV, Korhonen PK, Young ND, Scott JB et al. 2019 - Comparative genome analysis indicates high evolutionary potential of pathogenicity genes in Colletotrichum tanaceti. PLoS One 14, e.0212248. 
Leon AMM, Hernandez WS, Perez LG, Crespo K et al. 2018 - First report of leaf anthracnose caused by Colletotrichum grossum on mango (Mangifera indica) in Cuba. Journal of Plant Pathology 100, 329.

Liang X, Wang B, Dong Q, Li L et al. 2018 - Pathogenic adaptations of Colletotrichum fungi revealed by genome wide gene family evolutionary analyses. PloS one 13, e0196303.

Li H, Zhou GY, Qi XY, Jiang SQ. 2018 - First report of Colletotrichum henanense causing anthracnose on tea-oil trees in China. Plant Disease 102, 1040.

Li Q, Mo J, Guo T, Huang S et al. 2016a - First report of leaf spot of Curcuma wenyujin caused by Colletotrichum curcumae in China. Plant Disease 100, 521-522.

Li GJ, Hyde KD, Zhao RL, Hongsanan S et al. 2016b - Fungal diversity notes 253-366: taxonomic and phylogenetic contributions to fungal taxa. Fungal Diversity 78, 1-237.

Li SZ, Li H. 2020 - Colletotrichum numphaeae causing anthracnose on Camellia oleifera in China. Plant Disease 104, 1860.

Lichtemberg PSF, Moral J, Morgan DP, Felts DG et al. 2017 - First report of anthracnose caused by Colletotrichum fioriniae and C. karstii in California pistachio orchards. Plant Disease 101, 1320.

Lin CH, Long XP, Li ZP, Zhang Y et al. 2020 - First report of anthracnose of Clausena lansium caused by Colletotrichum scovillei in China. Plant Disease 104, 1557.

Lin CH, Wu WQ, Liao XM, Liu WB et al. 2018 - First report of leaf anthracnose caused by Colletotrichum alatae on water yam (Dioscorea alata) in China. Plant Disease 102, 248.

Lindquist JC. 1948 - Notas Del Museo de La Plata 13, 40.

Ling JF, Peng A, Jiang Z, Xi P et al. 2020 - First Report of Anthracnose Fruit Rot Caused by Colletotrichum fioriniae on Litchi in China. Plant Disease.

Doi 10.1094/PDIS-07-20-1539-PDN

Liu B, Stein L, Cochran K, du Toit LJ et al. 2020 - Characterization of leaf spot pathogens from several spinach production areas in the United States. Plant Disease 104, 1994-2004.

Liu F, Cai L, Crous PW, Damm U. 2013a - Circumscription of the anthracnose pathogens Colletotrichum lindemuthianum and C. nigrum. Mycologia 105, 844-860.

Liu F, Cai L, Crous PW, Damm U. 2014 - The Colletotrichum gigasporum species complex. Persoonia 33, 83-97.

Liu F, Damm U, Cai L, Crous PW. 2013b - Species of the Colletotrichum gloeosporioides complex associated with anthracnose diseases of Proteacea. Fungal Diversity 61, 89-105.

Liu F, Hyde KD, Cai L. 2011 - Neotypification of Colletotrichum coccodes, the causal agent of potato black dot disease and tomato anthracnose. Mycology 2, 248-254.

Liu F, Tang G, Zheng X, Li Y et al. 2016a - Molecular and phenotypic characterization of Colletotrichum species associated with anthracnose disease in peppers from Sichuan Province, China. Scientific Reports 6, 1-17.

Liu F, Wang M, Damm U, Crous PW, Cai L. 2016c - Species boundaries in plant pathologenic fungi: a Colletotrichum case study. BMC Evolutionary Biology 16, 1-4.

Liu F, Weir BS, Damm U, Crous PW et al. 2015a - Unravelling Colletotrichum species associated with Camellia, employing apmat and $g s$ loci to resolve species in the C. gloeosporioides complex. Persoonia 35, 63.

Liu HN, Liu JA, Zhou GY. 2020a - First report of Colletotrichum alienum causing anthracnose on Aquilaria sinensis in China. Plant Disease 104, 283.

Liu JK, Hyde KD, Jones EBG, Ariyawansa HA et al. 2015b - Fungal diversity notes 1-110: taxonomic and phylogenetic contributions to fungal species. Fungal Diversity 72, 1-197.

Liu L, Zhang L, Qiu P, Wang Y et al. 2020b - Leaf spot of Polygonatum odoratum caused by Colletotrichum spaethianum. Journal of Genetics in Plant Pathology 86, 157-161.

Liu LP, Yang LY, Liu YN, Lu BH et al. 2016b - First report of anthracnose disease caused by Colletotrichum fioriniae on barbary wolfberry in China. Plant Disease 100, 2534.

Liu M, Zhang W, Zhou Y, Liu Y et al. 2016d - First report of twig anthracnose on grapevine caused by Colletotrichum nymphaeae in China. Plant Disease 100, 2530. 
Liu X, Xie X, Duan J. 2007 - Colletotrichum yunnanense sp. nov., a new endophytic species from Buxus sp. Mycotaxon 100, 137-144.

Liu YL, Lu JN, Zhou YH. 2019 - First report of Colletotrichum truncatum causing anthracnose of lucky bamboo in Zhanjiang, China. Plant Disease 103, 2947-2948.

Liu YN, Mao FR, Zhang H, Wang LB et al. 2018 - Colletotrichum cymbidiicola causes leaf spot of Cymbidium ensifolium in Jilin Province, China. Plant Disease 102, 1462-1463.

Lobik AI. 1928 - Materialy k mikologicheskoj flore Terskogo okruga [Material to the mycological flora of the Tersk Region]. Bolezni Rastenij. 17, 157-208.

Lobik AI. 1933 - Trudy severo-kavkazskogo Instituta Zashchity Rastenii 1, 39.

Lu GZ, Cannon PF, Reid A, Simmons CM. 2004 - Diversity and molecular relationships of endophytic Colletotrichum isolates from the Iwokrama Forest Reserve, Guyana. Mycological Research 108, 53-63.

Lyu H, Li C. 2020 - First report of anthracnose caused by Colletotrichum americae - borealis on greenhouse-grown licorice in China. Plant Disease 104, 1559.

Ma X, Nontachaiyapoom S, Jayawardena RS, Hyde KD et al. 2018 - Endophytic Colletotrichum species from Dendrobium spp. in China and Northern Thailand. MycoKeys 43, 23-57.

Macedo DM, Pereira OL, Hora Junior BT, Weir BS et al. 2016 - Mycobiota of the weed Tradescantia fluminensis in its native range in Brazil with particular reference to classical biological control. Australasian Plant Pathology 45, 45-56.

MacKenzie SJ, Seijo TE, Legard DE, Timmer PW, Peres NA. 2007 - Selection for pathogenicity to strawberry in populations of Colletotrichum gloeosporioides from native plants. Phytopathology 97, 1130-1140.

Maffei. 1921 - Riv. Patol. veget. 11, 116.

Magnaghi. 1902 - Atti Reale Ist. Bot. Univ. Pavia 8, 11.

Maharachchikumbura SSN, Hyde KD, Jones EBG, McKenzie EHC et al. 2015 - Towards a natural classification and backbone tree for Sodariomycetes. Fungal Diversity 72, 199-301.

Maharachchikumbura SN, Hyde KD, Jones EBG, McKenzie EHC et al. 2016 - Families of Sordariomycetes. Fungal Diversity 79, 1-317.

Maire RCJE. 1913 - Études Mycologiques, Fasc. 1. Annales Mycologici 11, 331-358.

Maire RCJE. 1917 - Champignons nord-Africains nouveaux ou peu connus. Bulletin de la Société d'Histoire Naturelle de 1'Afrique du Nord. 8, 134-200.

Manamgoda DS, Udayanga D, Cai L, Chukeatirote E et al. 2013 - Endophytic Colletotrichum from tropical grasses with a new species Colletotrichum endophytica. Fungal Diversity 61, 107115.

Marcelino J, Giordano R, Gouli S, Gouli V et al. 2008 - Colletotrichum acutatum var. foriniae (teleomorph: Glomerella acutata var. foriniae var. nov.) infection of a scale insect. Mycologia 100, 353-374.

Marin-Felix Y, Groenewald JZ, Cai L, Chen Q et al. 2017 - Genera of phytopathogenic fungi, GOPHY 1. Studies in Mycology 86, 99-216.

Masel A, Braithwaite K, Irwin J, Manners J. 1990 - Highly variable molecular karyotypes in the plant pathogen Colletotrichum gloeosporioides. Current Genetics 18, 81-86.

Massee GE. 1906 - Fungi exotici, V. Bulletin of Miscellaneous Informations of the Royal Botanical Gardens Kew, 256-258.

Massee GE. 1912 - Fungi exotici, XIII. Bulletin of Miscellaneous Informations of the Royal Botanical Gardens Kew, 189-191.

Massee GE. 1913 - Kew Bulletin of Miscellaneous Information, 198.

Massenot. 1961 - Bulletin Res. Counc. Israel, 10D 10, 194.

Matos KS, Machado JF, Chagas PC, Siqueira RHS et al. 2020 - First report of Colletotrichum aeschynomenes and C. tropicale causing anthracnose on Myrciaria dubia in Brazil. Plant Disease 104, 2517. 
Matos KS, Santana KFA, Catarino AM, Hanada RE et al. 2017 - First report of anthracnose on welsh onion (Allium fistulosum) in Brazil caused by Colletotrichum theobromicola and C. truncatum. Plant Disease101, 1055-1056.

Matsushima T. 1996 - Matsushima Mycological Memoirs 9. Matsushima Mycological Memoirs. 9, $1-30$.

Maublanc A, Rangel E. 1915 - Alguns fungos do Brasil, novos ou mal conhecidos. Boletim de Agricultura São Paulo. 16, 310-328.

Meetum P, Leksomboon C, Kanjanamaneesathian M. 2015 - First report of Colletotrichum aenigma and $C$. siamense, the causal agents of anthracnose disease of dragon fruit in Thailand. Journal of Plant Pathology 97, 402.

Mendgen K, Hahn M. 2002 - Plant infection and the establishment of fungal biotrophy. Trends in Plant Science 7, 352-356.

Mills PR, Hodson A, Brown AE. 1992 - Molecular differentiation of Colletotrichum gloeosporioides isolates infecting tropical fruits. In: Colletotrichum: Biology, Pathology and Control (Bailey JA, Jeger MJeds). CABI, Wallingford, UK: 269-288.

Moesz G. 1931 - Mykológiai közlemények. VIII. közlemény. Botanikai Kölzlemények 28, 161174.

Montri P, Taylor PWJ, Mongkolporn O. 2009 - Pathotypes of Colletotrichum capsici, the causal agent of chilli anthracnose in Thailand. Plant Disease 93, 17-20.

Moraes SRG, Tanaka FAO, Massola Júnior NS. 2013 - Histopathology of Colletotrichum gloeosporioides on guava fruits (Psidium guajava L.). Rev Bras Frutic 35, 657-664.

Moreira RR, Peres NA, May De Mio LL. 2019 - Colletotrichum acutatum and C. gloeosporioides species complexes associated with apple in Brazil. Plant Disease 103, 268-275.

Morgan-Jones G. 1971 - Conidium ontogeny in Coelomycetes. II. Some Melanconiales which possess phialides. Canadian Journal of Botany 49, 1931-1937.

Moriwaki J, Tsukiboshi T. 2009 - Colletotrichum echinochloae, a new species on Japanese barnyard millet (Echinochloa utilis). Mycoscience 50, 273-280.

Moriwaki J, Sato T, Tsukiboshi T. 2003 - Morphological and molecular characterization of Colletotrichum boninense sp. nov. from Japan. Mycoscience 44, 47-53.

Mundkur. 1938 - Sci. Monogr. Coun. Agric. Res. India 12, 38.

Münch S, Lingner U, Floss DS, Ludwig N et al. 2008 - The hemibiotrophic lifestyle of Colletotrichum species. Journal of Plant Physiology 165, 41-51.

Muthappa BN. 1967 - Fungi of Coorg (India). II. Sydowia 21, 154-158.

Mzhavanadze. 1963 - Soobshch. Akad. Nauk. Gruz. SSR 31, 146.

Nakamura M, Ohzono M, Kei-Arai HS. 2006 - Anthracnose of Sansevieria trifasciata caused by Colletotrichum sansevieriae sp. nov. Journal of General Plant Pathology 72, 253-256.

Namyslowski B. 1906 - Zapiski mykologiczne. Sprawozdanie Komisji Fizjograficznej 39, 70-86.

Narendra DV, Rao VG. 1972 - Some additions to the fungi of India. Sydowia 26, 282-284.

Nascimento AD, Lima MO, Feijo FM, Junior JH et al. 2019 - First report of Colletotrichum aeschynomenes causing anthracnose in cacao (Theobroma cacao) in Brazil. Plant Disease $103,3284$.

Nasheshi A, Kadir J, Rashid TS, Awla HK et al. 2016 - Occurrence of anthracnose fruit rot caused by Colletotrichum nymphaeae on pepper (Capsicum annuum) in Malaysia. Plant Disease 100, 1244.

Neergaard. 1943 - Aarsberetn. J. E. Ohlens Enkesplantepatol. Lab. 1 April 1942-31 Marts 8.

Negru. 1958 - Stud. Cercet. Biol. Acad. romana 9, 34.

Negru. 1959 - Stud. Cercet. Biol. Acad. romana 11, 384.

Negru. 1961 - Notulae Syst. Sect. Crypt. Inst. Bot. Acad. Sci. U.S.S.R. 14, 186.

Newton AC, Fitt BD, Atkins SD, Walters DR, Daniell TJ. 2010 - Pathogenesis, parasitism and mutualism in the trophic space of microbe-plant interactions. Trends in Microbiology 18, 365-373. 
Nguyen HDT, JančičS, Meijer M, Tanney JB et al. 2015 - Application of the phylogenetic species concept to Wallemia sebi from house dust and indoor air revealed by multi-locus genealogical concordance. PLoS One 10, 120894.

Nicolas, Aggéry N. 1942 - Bull. Mém. Soc. Med. Hôp. Paris 37.

Niu X, Gao H, Qi J, Chen M et al. 2016 - Colletotrichum species associated with jute (Corchorus capsularis L.) anthracnose in southeastern China. Scientific Reports 6, 1-9.

Noack F. 1897 - Bol. Inst. Agron. Estado Sao Paulo 9, 80.

Noack F. 1901 - Die Krankheiten des Kaffeebaumes in Brasilien. III. Colletotrichum coffeanum n. sp. Zeitschrift für Pflanzenkrankheiten und Pflanzenschutz. 2, 196-203.

Noireung P, Phoulivong, Liu F, Cai L et al. 2012 - Novel species of Colletotrichum revealed by morphology and molecular analysis. Cryptogamie Mycology 33, 347-362.

Nolla JAB. 1926 - Onion-leaf anthracnose. Journal of the Department of Agriculture of Porto Rico $10,245-256$.

Noor NM, Zakaria L. 2018 - Identification and characterization of Colletotrichum spp. associated with chili anthracnose in peninsular Malaysia. European Journal of Plant Pathology 151, 961-973.

Novosselova. 1938 - Colletotrichum acanthostachydis. Notul. Syst. Sec. Cryptog. Ins. Bot. Acd. Sci. USSR. 4, 39.

Ntahimpera N, Madden LV, Wilson LL. 1997 - Effect of rain distribution alteration on splash dispersal of Colletotrichum acutatum. Phytopathology 87, 649-655.

O'Connell RJ, Thon MR, Hacquard S, Amyotte SG et al. 2012 - Lifestyle transitions in plant pathogenic Colletotrichum fungi deciphered by genome and transcriptome analyses. Nature Genetics 44, 1060-1065.

O’Donnell K, Sutton DA, Fothergill A, McCarthy D et al. 2008 - Molecular phylogenetic diversity, multilocus haplotype nomenclature, and in vitro antifungal resistance within the Fusarium solani species complex. Journal of Clinical Microbiology 46, 2477-2490.

O'Gara PJ. 1915 - New species of Colletotrichum and Phoma. Mycologia 7, 38-41.

Okorley BA, Sossah FL, Dan D, Li Y et al. 2019 - First report of Colletotrichum spaethianum causing anthracnose on Anemarrhena asphodeloides in China. Plant Disease 103, 1414-1415.

Oo MM, Lim GT, Jang HA, Oh SK. 2017 - Characterization and pathogenicity of new record of anthracnose on various chili varieties caused by Colletotrichum scovillei in Korea. Mycobiology 45, 184-191.

Oo MM, Oh SK. 2017 - Identification and characterization of new record of grape ripe rot disease caused by Colletotrichum viniferum in Korea. Mycobiology 45, 421-425.

Oo MM, Tweneboah S, Oh SK. 2016 - First report of anthracnose caused by Colletotrichum fioriniae on Chinese matrimony vine in Korea. Mycobiology 44, 325-329.

Pal N, Testen AL. 2020 - First report of quinoa anthracnose caused by Colletotrichum nigrum and C. truncatum in the United States. Plant Disease. Doi 10.1094/PDIS-07-20-1568-PDN

Parmelee JA. 1958 - Some foliicolous fungi of the Pyrolaceae. Canadian Journal of Botany 36, 865-881.

Passerini G. 1888 - Diagnosi di funghinuovi. Nota IV. Attidella Reale Accademiadei Lincei. Rendiconti di classe di Scienze Fisiche, Matematiche e Naturale. Ser. 4, 95-105.

Patel K, Pande CB. 1953 - Indian Phytopath. 5, 136.

Patouillard NT, Lagerheim G de. 1891 - Champignons de 1'Equateur (Pugillus I). Bulletin de la Société Mycologique de France 7, 158-184.

Patterson W. 1900 - New species of fungi. Bulletin of the Torrey Botanical Club 27, 282-286.

Patwardhan PG. 1966 - A new species of Colletotrichum and its associated ascigerous stage. Mycopathologia et Mycologia Applicata 29, 365-368.

Patwardhan PG, Sathe AV. 1965 - Some new records of Fungi Imperfecti from India. Sydowia 19, $150-155$. 
Pavgi MS, Singh UP. 1964 - Parasitic fungi from north India. III. Mycopathologia et Mycologia Applicata 24, 355-361.

Pavgi MS, Singh UP. 1965 - Parasitic fungi from North India IV. Mycopathologia et Mycologia Applicata 27, 81-88.

Pavgi MS, Upadhyay HP. 1964 - Parasitic fungi from north India. II. Mycopathologia et Mycologia Applicata 24, 347-354.

Peck CH. 1902 - Report of the State Botanist (1901). Annual Report on the New York State Museum of Natural History 54, 929-984.

Peng LJ, Sun T, Yang YL, Cai L et al. 2013 - Colletotrichum species on grape in Guizhou and Yunnan provinces, China. Mycoscience 54, 29-41.

Penzig, Saccardo. 1884 - Atti Inst. Veneto Sci. lett., ed Arti 2, 577.

Perfect SE, Hughes HB, O’Connell RJ, Green JR. 1999 - Colletotrichum: a model genus for studies on pathology and fungal-plant interactions. Fungal Genetics and Biology 27, 186198.

Petch T. 1906 - Descriptions of new Ceylon fungi. Annals of the Royal Botanic Gardens Peradeniya 3, 1-10.

Petch T. 1917 - Additions to Ceylon fungi. Annals of the Royal Botanic Gardens Peradeniya 6, $195-256$.

Petch T. 1924 - Revisions of Ceylon fungi (Part VII). Annals of the Royal Botanic Gardens Peradeniya 9, 119-184.

Pethybridge GH. 1919 - Notes on some saprophytic species of fungi associated with diseased potato plants and tubers. Transactions of the British Mycological Society 6, 104-120.

Peres NA, Timmer LW, Adaskaveg JE, Correll JC. 2005 - Lifestyles of Colletotrichum acutatum. Plant Disease 89, 784-796.

Petrak F. 1925 - Mykologische Notizen. VIII. Annales Mycologici 23, 1-143.

Petrak F. 1927 - Mykologische Notizen. IX. Annales Mycologici 25, 193-343.

Petrak F. 1929 - Mykologische Notizen. X. Annales Mycologici 27, 324-410.

Petrak F. 1934 - Mykologische Notizen. XII. Annales Mycologici 32, 317-447.

Petrak F. 1939 - Just's Bot. Jahresber. 56, 355.

Petrak F. 1947a - Kritische Studienüber Chinesische Pilze. Sydowia 1, 332-377.

Petrak F. 1947b - Sudamerikanische Mikromyzeten. Sydowia 1, 289-308.

Petrak F. 1956 - Mykologische Bemerkungen. Sydowia 10, 296-305.

Petrak F, Ciferri R. 1932 - Fungi Dominicani. II. Annales Mycologici 30, 149-353.

Petrak F, Sydow H. 1924 - Kritisch-systematische Originaluntersuchungen über Pyrenomyzeten, Sphaeropsideen und Melanconieen. Annales Mycologici 22, 318-386.

Petrak F, Sydow H. 1925 - Kritisch-systematische Originaluntersuchungen über Pyrenomyzeten, Sphaeropsideen und Melanconieen. Annales Mycologici 23, 209-294.

Petrak F, Sydow H. 1926 - Die Gattungen der Pyrenomyzeten, Sphaeropsideen und Melanconieen.1. Die phaeosporen Sphaeropsideen und die Gattung Macrophoma. Feddes Repertorium Spec. Nov. Regni Vegetabilum Beihefte 42, 1-551.

Petrak F, Sydow H. 1934 - Kritisch-systematische Originaluntersuchungen über Pyrenomyceten, Sphaeropsideen und Melanconieen. VI. Annales Mycologici 33, 157-193.

Photita W, Lumyong S, Lumyong P, Hyde KD. 2001 - Endophytic fungi of wild banana (Musa acuminata) at Doi Suthep Pui National Park, Thailand. Mycological Research 105, 15081513.

Photita W, Lumyong S, Lumyong P, McKenzie EHC, Hyde KD. 2004 - Are some endophytes of Musa acuminata latent pathogens? Fungal Diversity 16, 131-140.

Phoulivong S, Cai L, Parinn N, Chen H et al. 2010 - A new species of Colletotrichum from Cordyline fruticosa and Eugenia javanica causing anthracnose disease. Mycotaxon 114, 247257. 
Pinto JMA, Pereira R, Mota SF, Ishikawa FH et al. 2019 - Investigating phenotypic variability in Colletotrichum lindemuthianum populations. Phytopathology 102, 490-497.

Poletto T, Muniz MFB, Blume E, Fantinel VS et al. 2019 - First report of Colletotrichum numphaeae causing anthracnose on Carya illinoinensis in Brazil. Plant Disease 103, 3277.

Politis JC. 1935 - Contribution à l'étude des champignons de l'Attique. Pragmateiai tes Akademias Athenon. 4, 1-44.

Pollacci. 1896 - Atti dell'Istituto Botanico della R. Universita di Pavia, II 5, 16.

Posada D. 2002 - Evaluation of methods for detecting recombination from DNA sequences, empirical data. Molecular Biology and Evolution 19, 708-717.

Prihastuti H, Cai L, Chen H, McKenzie EHC et al. 2009 - Characterization of Colletotrichum species associated with coffee berries in northern Thailand. Fungal Diversity 39, 89-109.

Prihastuti H, Cai L, Crouch JA, Phoulivong S et al. 2010 - Neotypification of Colletotrichum falcatum, the causative agent of red-rot disease in sugarcane. Sydowia 62, 283-293.

Prins TW, Tudzynski P, von Tiedemann A, Tudzynski B. 2000 - Infection strategies of Botrytis cinerea and related necrotrophic pathogens. Fungal pathology, Springer, Dordrecht 33-64.

Promputtha I, Lumyong S, Vijaykrishna D, McKenzie EHC et al. 2007 - A phylogenetic evaluation of whether endophytes become saprotrophs at host senescence. Microbial Ecology 53, 579590.

Prusky D. 1996 - Pathogen quiescence in postharvest diseases. Annual Reviews of Phytopathology 34, 413-434.

Prusky D, Alkan N, Mengiste T, Fluhr R. 2013 - Quiescent and nectrophic lifestyle choice during postharvest disease development. Annual Reviews of Phytopathology 51, 155-176.

Prusky D, Plumbley RA. 1992 - Quiescent infections of Colletotrichum in tropical and subtropical fruits. CAB International, Wallington, UK.

Pszczolkowska A, Okorski A, Paukszto L, Jastrzebski J. 2016 - First report of anthracnose disease caused by Colletotrichum fioriniae on blueberry in Western Poland. Plant Disease 100, 2167.

Qin LP, Zhang Y, Su Q, Chen YL et al. 2019 - First report of anthracnose of Mangifera indica caused by Colletotrichum scovillei in China. Plant Disease 103, 1043-1044.

Quaedvlieg W, Binder M, Groenewald JZ, Summerell BA et al. 2014 - Introducing the Consolidated Species Concept to resolve species in the Teratosphaeriaceae. Persoonia 33, 140.

Quimio TH. 1977 - Species of Colletotrichum in the Philippines. Nova Hedwigia 28, 543-553.

Radulescu E, Negru. 1963 - Studii si Cercetari de Biololie. 15, 390.

Rakotoniriana EF, Scauflaire J, Rabemanantsoa C, Urveg-Ratsimamanga S et al. 2013 Colletotrichum gigasporum sp. nov., a new species of Colletotrichum producing long straight conidia. Mycological Progress 12, 403-412.

Ramakrishnan TS. 1951 - Additions to fungi of Madras - X. Proceedings of the Indian Academy of Sciences Section B 34, 63-72.

Ramakrishnan TS, Ramakrishnan K. 1947 - Additions to fungi of Madras - II. Proceedings of the Indian Academy of Sciences Section B 25, 178-186.

Ranathunge NP, Mongkolporn O, Ford R, Taylor PWJ. 2012 - Colletotrichum truncatum pathosystems on Capsicum spp: infection, colonization and defence mechanisms. Australasians Plant Pathology 41, 463-473.

Rangel. 1915 - International Agriculture.-technology. Rundschau 6, 1213.

Rangel. 1926 - Instituto Geral de Perícias de Santa Catarina 2, 3-34.

Rao V. 1963 - Bulletin of botanical Society of Collage of Science, Nagpur 4, 56.

Rao, Salam MS. 1960 - Journal of Indian botanical Society 39, 324.

Réblová M, Gams W, Seifert KA. 2011 - Monilochaetes and allied genera of the Glomerellales, and a reconsideration of families in the Microascales. Studies in Mycology 68, 163-191.

Redman RS, Dunigan DD, Rodriguez RJ. 2001 - Funagl symbiosis from mutualism to parasitism: who controls the outcome, host or invader?. New Phytologist 151, 705-716. 
Renner MA, Heslewood MM, Patzak SD, Schäfer-Verwimp A, Heinrichs J. 2017 - By how much do we underestimate species diversity of liverworts using morphological evidence? An example from Australasian Plagiochila (Plagiochilaceae: Jungermanniopsida). Molecular Phylogenetics and Evolution 107, 576-593.

Roberts RG, Snow JP. 1990 - Morphological and pathological studies of Colletotrichum capsici and $C$. indicum. Mycologia 82, 82-90.

Rodriguez RJ, Redman RS. 2000 - Colletotrichum as a model system for defining the genetic basis of fungal symbiotic lifestyles. In: Colletotrichum. Host Specificity, Pathology and HostPathogen Interaction (Prusky D, Freeman S, Dickman MB eds). APS Press, St Paul, USA: 114-130.

Rodriguez-Guerra R, Ramírez-Rueda MT, Cabral-Enciso M, García-Serrano M et al. 2005 Heterothallic mating observed between Mexican isolates of Glomerella lindemuthiana. Mycologia 97, 793-803.

Rogério F, Ciampi-Guillardi M, Barbieri MCG, Bragança CAD et al. 2017 - Phylogeny and variability of Colletotrichum truncatum associated with soybean anthracnose in Brazil. Journal of Applied Microbiology 122, 402-415.

Rojas EI, Rehner SA, Samuels GJ, Van Bael SA et al. 2010 - Colletotrichum gloeosporioides sl associated with Theobroma cacao and other plants in Panama: multilocus phylogenies distinguish host-associated pathogens from asymptomatic endophytes. Mycologia 102, 13181338 .

Roldan. 1936 - Philippine Journal of Science 60, 120.

Roy. 1948 - Journal of Indian botanical Society 27, 101.

Saccardo PA. 1882 - Fungi Gallicilecti a Cl. viris P. Brunaud, C.C. Gillet, Abb.Letendre, A. Malbranche, J. Therry \& Dom. Libert. Series IV. Michelia 2, 583-648.

Saccardo PA. 1884 - Sylloge Fungorum, Sylloge Sphaeropsidearum et Melanconiearum. Sylloge Fungorum. 3, 1-840.

Saccardo PA. 1902 - Florae MycologicaeLusitanicae. Contributioduodecima. Boletim da Sociedade Broteriana. 19, 156-171.

Saccardo PA. 1908 - Sylloge Fungorum XIX, 369.

Saccardo PA. 1906 - Sylloge Fungorum (Abellini) 18, 466.

Saccardo PA. 1912 - Notaemycologicae. Series XIV. Annales Mycologici 10, 310-322.

Saccardo PA. 1914 - Notaemycologicae. Series XVIII. Annales Mycologici 12, 282-314.

Saccardo PA. 1915 - Fungi ex insula Melita (Malta) lecti a Doct. Caruana-Gatto et Doct. G. Borg annis MCMXIII et MCMIV. Nuovo Giornale Botanico Italiano 22, 24-76.

Saccardo PA. 1921 - Notaemycologicae. Ser. XXVII. Fungi sinenses aliquot a cl. Prof. Otto A. Reinkingcollecti et communicati. Philippine Journal of Science Section C Botany 18, 595605.

Saccardo PA. 1922 - Sylloge Fungorum XXV, 578

Saccardo PA, Sydow P. 1899 - Sylloge Fungorum 14, 1016

Saccardo PA, Trotter A. 1913 - Supplementum Universale, Pars IX. Sylloge Fungorum 22, 11612.

Sahni VP. 1968 - Deuteromycetes from Jabalpur. III. Mycopathologia et Mycologia Applicata. 36, 267-288.

Salunkhe VN, Anandhan S, Gawande SJ, Ikkar RB et al. 2018a - First report of Colletotrichum truncatum causing anthracnose of mouse garlic (Allium angulosum) in India. Plant Disease $102,240$.

Salunkhe VN, Anandhan S, Gawande SJ, Ikkar RB et al. 2018b - First report of anthracnose caused by Colletotrichum spaethianum on Allium ledebourianum in India. Plant Disease 102, 2031.

Samarakoon MC, Persoh D, Hyde KD, Bulgakov TS et al. 2018 - Colletotrichum acidae sp. nov. from northern Thailand and a new record of $C$. dematium on Iris sp. Mycosphere 9, 583-597. 
Sanders GM, Korsten L. 2003 - Comparison of cross inoculation potential of South African avocado and mango isolates of Colletotrichum gloeosporioides. Microbiological Research $158,143-150$.

Sandu. 1959a - Lejeunia Mém., 838

Sandu. 1959b - Lejeunia Mém., 840

Sandu-Ville C. 1962 - Stud. Cercet Stiint. Iasi, Biol. Stiinte agric. 13, 266.

Sangpueak R, Phansak P, Buensanteai N. 2018 - Morphological and molecular identification of Colletotrichum species associated with cassava anthracnose in Thailand. Journal of Phytopathology 166, 129-142.

Santana KFA, Garcia CB, Matos KS, Hanada RE et al. 2016 - First report of anthracnose caused by Colletotrichum spaethianum on Allium fistulosum in Brazil. Plant Disease 100, 224-225.

Sato T, Moriwaki J. 2013 - Molecular re-identification of strains in NIAS Genebank belonging to phylogenetic groups A2 and A4 of the Colletotrichum acutatum species complex. Microbial Resources and Systematics (Microbiology and Culture Collections) 29, 13-23.

Sato T, Moriwaki J, Uzuhashi S, Degawa Y et al. 2012 - Molecular phylogenetic analyses and morphological re-examination of strains belonging to three rare Colletotrichum species in Japan. Microbiology and Culture Collections 28, 121-134.

Savian LG, Muniz MFB, Poletto T, Maculan LG et al. 2019 - First report of Colletotrichum nymphaeae causing anthracnose on Juglans regia fruits in sourthern Brazil. Plant Disease $103,3287$.

Savulescu T, Negru. 1955 - Herb. mycologicum Romanicum 31, no. 1539.

Savulescu T, Sandu-Ville C. 1933 - Beitragzur Kenntnis der Micromyceten Rumäniens. Hedwigia. 73, 71-132.

Sawada K. 1933 - Descriptive catalogue of the Formosan fungi VI. Report of the Department of Agriculture Government Research Institute of Formosa. 61, 1-117.

Sawada K. 1943 - Descriptive catalogue of the Formosan fungi. Part VIII. Report of the Department of Agriculture Government Research Institute of Formosa. 85, 1-130.

Sawada K. 1944 - Descriptive catalogue of Formosan fungi. Report of the Department of Agriculture Government Research Institute of Formosa. 87, 1-96.

Sawada K. 1958 - Researches on fungi in Tohoku District of Japan. (IV). Fungi Imperfecti. Bulletin of the Government Forest Experimental Station Meguro. 105, 35-140.

Sawada K. 1959 - Descriptive catalogue of Taiwan (Formosan) fungi. Part XI. Special Publication College of Agriculture National Taiwan University. 8, 1-268.

Sawada K. 1965 - Lich. Foliic. Leg. Beccari, 45.

Scaramella. 1932 - Colletotrichum veronicae Malpighia 32, 219.

Schena L, Mosca S, Cacciola SO, Faedda R et al. 2014 - Species of the Colletotrichum gloeosporioides and $C$. boninense complexes associated with olive anthracnose. Plant Pathology 63, 437-446

Schulzer S, Saccardo PA. 1884 - Micromycetes Slavonicinovi V (69-84). Hedwigia 23, 125-128.

Schulze-Lefert P, Panstruga R. 2011 - A molecular evolutionary conceptconnecting non host resistance, pathogen host range and pathogen association. Trends in Plant Science 16, 117 125.

Serdani M, Rooney-Latham S, Wallis KM, Blomquist CL. 2013 - First report of Colletotrichum phormii causing anthracnose on New Zealand Flax in the United States. Plant Disease 97, 1115.

Sharma G, Maymon M, Freeman S. 2017 - Epidemiology, pathology and identification of Colletotrichum including a novel species associated with avocado (Persea americana) anthracnose in Israel. Scientific Report 1, 11-16.

Sharma G, Pinnaka AK, Shenoy BD. 2015 - Resolving Colletotrichum siamense species complex using apmat marker. Fungal Diversity 71, 247-264.

Sharma G, Shenoy BD. 2013 - Multigene sequence-based identification of Colletotrichum cymbidiicola, C. Karstii and C. Phyllanthi from India. Czech Mycology 65, 79-88. 
Sherriff C, Whelan MJ, Arnold GM, Lafay JF et al. 1994 - Ribosomal DNA sequence analysis reveals new species groupings in the genus Colletotrichum. Experimental Mycology 18, 121138.

Shi YP, Liu YX, Li LL, Dai LM et al. 2019 - First report of Colletotrichum laticiphilum causing anthracnose of Rubber tree in China. Plant Disease 103, 579.

Shivas RG, Bathgate J, Podger FD. 1998 - Colletotrichum xanthorrhoeae sp. nov. on Xanthorrhoea in Western Australia. Mycological Research 102, 280-282.

Shivas RG, Tan YP. 2009 - A taxonomic re-assessment of Colletotrichum acutatum, introducing C. fioriniae comb.et stat. nov. and C. simmondsii sp. nov. Fungal Diversity 39, 111-122.

Shivas RG, Tan YP, Edwards J, Dinh Q et al. 2016 - Colletotrichum species in Australia. Australasian Plant Pathology 45, 447-464.

Siemaszko W. 1923 - Badaniamykologiczne wgórachKaukazu. Arch. Nauk. Biol. Towarz. Nauk. Warszawa. 1, 1-54.

Simmonds JH. 1965 - A study of the species of Colletotrichum casuing ripe fruit rots in Queensland. Queensland Journal of Agricultural and Animal Science 25, 437-459.

Simmonds JH. 1968 - Type specimens of Colletotrichum gloeosporioides var. minor and Colletotrichum acutatum. Queensland Journal of Agricultural and Animal Science 25, 178A.

Silva DN, Talhinhas P, Várzea V, Cai L et al. 2012a - Application of the apn2/mat locus to improve the systematics of the Colletotrichum gloeosporioides complex: an example from coffee (Coffea spp.) hosts. Mycologia 104, 396-409.

Silva DN, Talhinhas P, Cai L, Manuel L et al. 2012b - Host-jump drives rapid and recent ecological speciation of the emergent fungal pathogen Colletotrichum kahawae. Molecular Ecology 21, 2655-2670.

Silva MD, Cruz ES, Veloso TG, Miranda L et al. 2018 - Colletorichum serranegrense sp. nov., a new endophytic species from the roots of the endangered Brazilian epiphytic orchid Cattleya jongheana. Phytotaxa 351, 163-170.

Sivanesan A, Hsieh WH. 1993 - A new ascomycete, Glomerella septospora sp. nov. and its coelomycete anamorph, Colletotrichum taiwanense sp. nov. from Taiwan. Mycological Research 97, 1523-1529.

Smith RE. 1899 - A new Colletotrichum disease of the pansy. Botanical Gazette Crawfordsville 27, 203-204.

Smith BJ, Black LL. 1990 - Morphological, cultural and pathogenic variation among Colletotrichum species isolated from strawberry. Plant Disease 74, 69-76.

Soares WRO, Quitania ACR, Miller RNG, Café-Filho AC et al. 2017 - First worldwide report of Colletotrichum pseudoacutatum causing anthracnose on Syzygium jambos. Plant Disease 101, 1322.

Soothworth EA.1891 - Antracnose of cotton. Journal of Mycology 6, 100-105.

Sousa da Câmara M. 1910 - Contributiones ad mycofloram Lusitaniae. Centuria VI. Boletim da Sociedade Broteriana. 25, 5-25.

Sousa da Câmara M. 1920 - Myc. novi mycofl. Lusitan, Revista agronomy Lisboa IV, 8.

Sousa da Câmara M. 1930 - Ann. Inst. Sup. Agron. Lisbosa. 3, 121.

Sousa da Câmara M. 1931a - Anais Inst. Super. Agron. Lisb. 4, 6 (extr.)

Sousa da Câmara M. 1931b -Revta agron., Lisb. 17, 10 [extr.]

Sousa da Câmara M. 1936 - Contributiones ad mycofloram Lusitaniae. Centuria XI. Boletim de Agricultura Lisboa. 2, 1-80.

Sousa-Dias de MR. 1958 - Colletotrichum tells-palhinhae n. sp. Boletim da Sociedade Broteriana. 32, 265-266.

Southworth EA. 1890 - A new hollyhock disease. Journal of Mycology 6, 45-50.

Spegazzini C. 1908 - Hongos de la yerba mate. Anales del Museo Nacional de Historia Natural Buenos Aires. 17, 111-141.

Spegazzini C. 1911 - Mycetes Argentinenses (Series V). Anales del Museo Nacional de Historia Natural Buenos Aires series 3 13, 329-467. 
Spegazzini C. 1918a - Fungi Costaricensesnonnulli. Boletín de la Academia Nacional de Cienciasen Córdoba. 23, 541-609.

Spegazzini C. 1918b - Reliquiaemycologicaetropicae. Boletín de la Academia Nacional de Cienciasen Córdoba. 23, 365-541.

Spegazzini C. 1920 - Algunas observacion es relativas a las hojas de coca (Erythroxylon coca

Lam.). Anales de la Sociedad Científica Argentina. 90, 23-32.

Spegazzini C. 1922 - Fungi Paraguayenses. Anales del Museo Nacional de Historia Natural Buenos Aires. 31, 355-450.

Sprague R. 1957 - Some leafspot fungi on Western Gramineae - XI. Mycologia 49, 837-853.

Sprague R. 1958 - Some leafspot fungi on western Gramineae - XII. Mycologia 50, 814-830.

Srivastava AK. 1969 - A new species of Colletotrichum on Gomphrena celosioides Mart. Sydowia 23, 118-119.

Srivastava SNS. 1953 - On the occurence of a new species of Colletotrichum on Pothos scandens L. Current Science 22, 244-244.

Sreenivasaprasad S, Brown AE, Mills PR. 1992 - DNA sequence variation and inter-relationship among Colletotrichum species causing strawberry anthracnose. Physiological and Molecular Plant Pathology 41, 265-281

Sreenivasaprasad S, Mills PR, Meehan BM, Brown AE. 1996 - Phylogeny and systematic of 18 Colletotrichum species based on ribosomal DNA spacer sequences. Genome 39, 499-512.

Sreenivasaprasad S, Talhinhas P. 2005 - Genotypic and phenotypic diversity in Colletotrichum acutatum, a cosmopolitan pathogen causing anthracnose on a wide range of hosts. Molecular Plant Pathology 6, 361-378.

Stajich JE. 2017 - Fungal genomes and insights into the evolution of the kingdom. The Fungal Kingdom 619-633.

Stergiopoulos I, Gordon TR. 2014 - Cryptic fungal infections: the hidden agenda of plant pathogens. Frontiers in Plant Science 5, article 506.

Stevens FL. 1917 - Porto Rican fungi, old and new. Transactions of the Illinois Academy of Science 10, 162-218.

Stevens FL. 1925 - Hawaiian fungi. Bulletin of the Bernice Bishop Museum 19, 1-189.

Stevens FL. 1927 - Fungi from Costa Rica and Panama. Illinois Biology Monographs 11, 157-255.

Stevens FL. 1930 - Parasitic fungi of British Guiana, Trinidad and Costa Rica. Annales Mycologici 28, 364-371.

Stevens FL, Hall JG. 1909 - Z. PflKrankh. PflSchutz 19, 68.

Stewart FC. 1900 - New York Agricultural Experiment Station Bulletin 179, 106.

Stewart JE, Timmer LW, Lawrence CB, Pryor BM et al. 2014 - Discord between morphological and phylogenetic species boundaries, incomplete lineage sorting and recombination results in fuzzy species boundaries in an asexual fungal pathogen. BMC Evolutionary Biology 14, 38 .

Stoneman. 1898 - Botanical Gazzet 26, 106.

Stukenbrock EH, McDonald BA. 2008 - The origins of plant pathogens in agro-ecosystems. Annual Review of Phytopathology 46, 75-100.

Sun WM, Wen XL, Qi HX, Feng LN et al. 2019 - First report of anthracnose of Atractylodes chinensis caused by Colletotrichum chlorophyti in China. Plant Disease 103, 764.

Sutton BC. 1966 - Development of fruitifications in Colletotrichum graminicola (Ces.) Wils. and related species. Canadian Journal of Botany 44, 887-897.

Sutton BC. 1968 - The appressoria of Colletotrichum graminicola and C. falcatum. Canadian Journal of Botany 46, 873-876.

Sutton BC. 1975 - Coelomycetes V. Coryneum. Mycological Papers. 138, 1-224.

Sutton BC. 1980 - The Coelomycetes. Fungi Imperfecti with Pycnidia, Acervuli and Stromata. Commonwealth Mycological Institute, Kew, Surrey, England, 1-696.

Sutton BC. 1992 - The genus Glomerella and its anamorph Colletotrichum. In: Colletotrichum: Biology, Pathology and Control (Bailey JA, Jeger MJ eds). CABI, Wallingford, UK: 1-26. 
Sutton TB, Shane WW. 1983 - Epidemiology of the perfect stage of Glomerella cingulata on apples. Phytopathology 73, 1179-1183.

Su YY, Noireung P, Liu F, Hyde KD et al. 2011 - Epitypification of Colletotrichum musae, the causative agent of banana anthracnose. Mycoscience 52, 376-382.

Sydow H. 1925a - Fungi from the province of Sorsogon. Leaflets of Philippine Botany. 9, 31133138 .

Sydow H. 1925b - Fungi in itinerecostaricensicollecti. Pars prima. Annales Mycologici. 23, 308429.

Sydow H. 1930 - Novae fungorum species - XX. Annales Mycologici. 28, 432-447.

Sydow H. 1932 - Annals mycology 30, 397.

Sydow H. 1935 - Novae fungorum species - XXIII. Annales Mycologici. 33, 367-384.

Sydow H, Mitter JH. 1933 - Fungi indici. I. Annales Mycologici. 31, 84-97.

Sydow H, Sydow P, Butler EJ. 1916 - Fungi Indiaeorientalis pars V. Annales Mycologici. 14,177220.

Sydow H, Sydow P. 1900 - Beiträgezur Kenntnis der Pilzflora der Mark Brandenburg. III. Hedwigia Beiblätter. 39, 1-6.

Sydow H, Sydow P. 1913 - Enumeration of Philippine fungi with notes and descriptions of new species, I, micromycetes. Philippine Journal of Science Section C Botany. 8, 265-285.

Sydow H, Sydow P. 1914a - Fungi from northern Palawan. Philippine Journal of Science Section C Botany 9, 157-189.

Sydow H, Sydow P. 1914b - Diagnose Neuer Philippinischer Pilze. Annales Mycologici 12, 545576.

Sydow H, Sydow P. 1939 - Fungi aequatorienses. Annales Mycologici. 37, 275-438.

Sydow P, Sydow H. 1920 - Weitereneue Micromyceten der Philippinen-Inseln. Annales Mycologici. 18, 98-104.

Talhinhas P, Sreenivasaprasad S, Neves-Martin J, Oliveira H. 2002 - Genetic and morpholological characterization of Colletotrichum acutatum causing anthracnose of lupins. Phytopathology 92, 986-996.

Talhinhas P, Sreenivasaprasad S, Neves-Martin J, Oliveira H. 2005 - Molecular and phenotypic analyses reveal association of diverse Colletotrichum acutatum groups and a low level of C. gloeosporioides with olive anthracnose. Applied and Environmental Microbiology 71, 2987-2998.

Tao G, Liu Zy, Liu F, Gao YH et al. 2013 - Endophytic Colletotrichum speciess from Bletilla ochracea (Orchidaceae), with descriptions of seven new species. Fungal Diversity 61, 139164.

Tassi F. 1899 - Novae micromycetum species descriptae et iconibus illustratae. VI [concl.]. Bollettino del Laboratorio de Orto Botanico Reale Universita Siena. 2, 231-232.

Tassi F. 1900 - Novae micromycetum species descriptae et iconibus illustratae. Bollettino del Laboratorio de Orto Botanico Reale Universita Siena. 3, 14-21.

Tassi F. 1904 - Nuovi Micromiceti. Bollettino del Laboratorio de Orto Botanico Reale Universita Siena. 6, 125-127.

Taubenhaus. 1916 - A contribution to our knowledge of silver scurf (Spondylocladiumatro virens Harz) of the white potato. Memoirs of the New York Botanical Garden. 6, 549-560.

Taylor JW, Jacobson DJ, Kroken S, Kasuga T et al. 2000 - Phylogenetic species recognition and species concepts in fungi. Fungal Genetics and Biology 31, 21-32.

Tehon LR, Daniels EY. 1925 - Notes on the parasitic fungi of Illinois. II. Mycologia. 17, 240-249.

Tehon LR. 1924 - Notes on the parasitic fungi of Illinois. Mycologia 16, 135-142.

Tehon LR. 1933 - Notes on the parasitic fungi of Illinois. Mycologia 25, 237-257.

Teng SC. 1936 - Additional fungi from China IV. Sinensia. 7,752-823

Than PP, Shivas RG, Jeewon R, Pongsupasamit S et al. 2008 - Epitypification and phylogeny of Colletotrichum acutatum J.H. Simmonds. Fungal Diversity 28, 97-108.

Tharp BC. 1917 - Texas parasitic fungi. Mycologia. 9, 105-124. 
Thite, Patil MS. 1975 - Botanique Nagpur 6, 114.

Tibpromma S, Hyde KD, Jeewon R, Maharachchikumbura SS et al. 2017 - Fungal Diversity Notes 491-602: taxonomic and phylogenetic contributions to fungal taxa. Fungal Diversity 83, 1261.

Tibpromma S, Hyde KD, Bhat JD, Mortimer PE et al. 2018 - Identification of endophytic fungi from leaves of Pandanaceae based on their morphotypes and DNA sequence data from southern Thailand. MycoKeys 33, 25.

Tilak ST. 1960 - A new species of Colletotrichum from an economic host. Current Science. 29, $147-147$.

Togashi K. 1936 - New species of parasitic fungi - I. Transactions of the Sapporo Natural History Society 14, 280-285.

Togashi, Onuma. 1934 - Bulletin of of the Imperial College of Agriculture and Forestry Morioka $17,47$.

Tognini. 1892 - Colletotrichum montemartinii Atti dell'Istituto Botanico della R. Universita di Pavia 3, 15 (extr.)

Tognini. 1895 - Colletotrichum ailanthi AttiIst. bot. 'Giovanni Briosi', ser. II (extr.), 16 (extr.)

Tóth A, Petróczy M, Palkovics L. 2017 - First report of Colletotrichum acutatumsensu lato through the occurrence of $C$. godetiae on Cornelian Cherry (Cornus mas) in Europe. Plant Disease $101,841-841$.

Udayanga D, Manamgoda DS, Liu X-Z, Chukeatirote E et al. 2013 - What are the common anthracnose pathogens of tropical fruits?. Fungal Diversity 61, 165-179.

Uematsu S, Kageyama K, Moriwaki J, Sato T. 2012 - Colletotrichum carthami comb. nov., an anthracnose pathogen of safflower, garland chrysanthemum and pot marigold, revived by molecular phylogeny with authentic herbarium specimens. Journal of General Plant Pathology 78, 316-330.

Underwood. 1891 - Colletotrichum adustum Journal of Mycology 7, 35.

Urríes MJ. 1952 - Notas micológicas. Anales del Jardín Botánico de Madrid. 10, 193-228.

van Kan JA. 2006 - Licensed to kill: the lifestyle of a necrotropic plant pathogen. Trends in Plant Science 11, 247-253.

Vaillancourt LJ, Hanau RM. 1991 - A method for genetic analysis of Glomerella graminicola (Colletotrichum graminicola) from maize. Phytopathology 81, 530-534.

Vaillancourt LJ, Hanau RM. 1992 - Genetic and morphological comparison of Glomerella (Colletotrichum) isolates from maize and from sorghum. Experimental Biology 16, 219-229.

Vargas WA, Martín JMS, Rech GE, Rivera LP et al. 2012 - Plant defense mechanism are activated during biotrophic and nectrotrophic development of Colletotrichum graminicola in maize. Plant Physiology 158, 1342-1358.

Vasquez-Lopez A, Palacios-Torres RE, Camacho-Tapia M, Granados-Echegoyen C et al. 2019 Colletotrichum brevisporum and C. musicola causing leaf anthracnose of taro (Colocasia esculenta) in Mexico. Plant Disease 103, 2963.

Vassiljevsky NI. 1940 - Notulae Syst. Sect. Crypt. Inst. Bot. Acad. Sci. U.S.S.R. 5, 17.

Vassiljevsky NI, Karakulin BP. 1950 - Fungi imperfecti Parasitici, Pars II. Melanconiales. ,1-680.

Vassiljevsky NI, Karakulin BP. 1926 - Fungi Imperfecti Parasitici 2, 303.

Velho AC, Alaniz S, Casanova L, Mondino P, Stadnik MJ. 2015 - New insights into the characterization of Colletotrichum species associated with apple diseases in southern Brazil and Uruguay. Fungal Biology 119, 229-244.

Verplancke, Claessens. 1934 - Colletotrichum gloriosae. Natuurwet. Tijdschr., 16, 17.

Viégas AP. 1946 - Alguns fungos do Brasil XII. Fungi Imperfecti. Bragantia. 6, 1-37.

Vieira WA, Lima WG, Nascimento ES, Michereff SJ et al. 2017 - The impact of phenotypic and molecular data on the inference of Colletotrichum diversity associated with Musa. Mycologia 109, 912-934.

Vieira WA, Michereff SJ, Jr de Morais MA, Hyde KD et al. 2014 - Endophytic species of Colletotrichum associated with mango in northeastern Brazil. Fungal Diversity 67, 181-202. 
Vinnere O, Fatehi J, Wright SA, Gerhardson B. 2002 - The causal agent of anthracnose of Rhododendron in Sweden and Latvia. Mycological Research 106, 60-69.

von Arx JA. 1957a - Revision der zu Gloeosporium gestellten Pilze. Verhandelingen Koninklijke Nederlandse Akademie van Wetenschappen Afdeling Natuurkunde. 51, 1-153.

von Arx JA. 1957b - Die Arten der Gattung Colletotrichum Cda. Phytopathologische Zeitschrift 29, 413-468.

Wakefield EM. 1922 - Fungi exotici. XXVI. Bulletin of Miscellaneous Informations of the Royal Botanical Gardens Kew. 5, 161-165.

Walker J, Nikandrow A, Millar GD. 1991 - Species of Colletotrichum on Xanthium (Asteraceae) with comments on some taxonomic and nomenclatural problems in Colletotrichum. Mycological Research 95, 1175-1193.

Walsh PD. 2000 - Sample size for the diagnosis of conservation units. Conservation Biology 14, $1533-1537$.

Waller JM, Bridge PD, Black R, Hakiza G. 1993 - Characterization of the coffee berry disease pathogen Colletotrichum kahawae sp. nov. Mycological Research 97, 989-994.

Wan Z, Liu JA, Zhou GY. 2018 - First report of Colletotrichum gigasporum causing anthracnose on Dalbergia odorifera in China. Plant Disease 102, 679.

Wang Y, Chen JY, Xu X, Cheng J et al. 2020 - Identification and characterization of Colletotrichum species associated with anthracnose disease of Camellia oleifera in China. Plant Disease 104, 474-482.

Wang Y, Qin HY, Liu YX, Fan ST et al. 2019 - First report of anthracnose caused by Colletotrichum aenigma on Actinidia arguta in China. Plant Disease 103, 372-373.

Wang YC, Hao XY, Wang L, Xiao B et al. 2016 - Diverse Colletotrichum species cause anthracnose of tea plants (Camellia sinensis (L.) O. Kuntze) in China. Scientific Reports 6, $1-13$.

Wang XM, Li JY. 1987 - Studies on species of Colletotrichum in Shaanxi Province, China. Acta Mycological Sinica 6, 211-218.

Waters JM, Rowe DL, Burridge CP, Wallis GP. 2010 - Gene trees versus species trees, reassessing life-history evolution in a freshwater fish radiation. Systematic Biology 59, 504-517.

Weimer. 1945 - Colletotrichum villosum. Phytopathology 35, 988.

Weir BS, Johnston PR. 2010 - Characterisation and neotypification of Gloeosporium kaki Hori as Colletotrichum horii nom. nov. Mycotaxon 111, 209-219.

Weir BS, Johnston PR, Damm U. 2012 - The Colletotrichum gloeosporioides species complex. Studies in Mycology 73, 115-180.

Wharton PS, Diéguez-Uribeondo J. 2004 - The biology of Colletotrichum acutatum. In Anales del jardín botánico de Madrid 61, 3-22.

Wharton PS, Schilder AC. 2008 - Novel infection strategies of Colletotrichum acutatum on ripe blueberry fruit. Plant pathology 57, 122-134.

Whitelaw-Weckert MA, Curtin SJ, Huang R, Steel CC et al. 2007 - Phylogenetic relationship and pathogenicity of Colletotrichum acutatum isolates from grape in subtropical Australia. Plant Pathology 56, 448-463.

Wikee S, Cai L, Pairin N, McKenzie EHC et al. 2011 - Colletotrichum species from Jasmine (Jasminum sambac). Fungal Diversity 46, 171-82.

Wilson GW. 1960 - Colletotrichum acalyphae Scientific Cultue 26, 87.

Woronichin NN. 1913 - Mycoflorae Caucasicae novitates. Vestnik Tiflissk. Botanicheskii Sada. $28,16-25$.

Wu CJ, Chen HK, Ni HF. 2020 - Identification and characterization of Colletotrichum species associated with mango anthracnose in Taiwan. European Journal of Plant pathology 157, 115.

Wu WP. 1992 - Notes on some coelomycetes on Euonymus japonicas I. Melanconiales. Journal of the Hebei Academy of Sciences 1, 65-74. 
Wu WX, Huang XQ, Liu Y, Zhang L. 2017 - First report of apple bitter rot caused by Colletotrichum rhombiforme in China. Plant Disease 101, 1033.

Wu WX, Liu Y, Huang XQ, Zhang L. 2018 - First report of anthracnose caused by Colletotrichum nymphaeae on loquat fruit in China. Plant Disease 102, 243.

Xavier KV, An Kc, Peres NA, Deng Z et al. 2019 - Characterization of Colletotrichum species causing anthracnose of pomegranate in South-eastern United States. Plant Disease 103, 27712780.

Xie L, Zhang JZ, Wang Y, Hu DW. 2010 - Identification of Colletotrichum spp. Isolated from strawberry in Zhejiang Province and Shanghai City, China. Journal of Zhejiang University Science B 11, 61-70.

Xu H, Zhou R, Fu J, Yuan Y et al. 2018a - Colletotrichum atractylodicola sp. nov., the anthracnose pathgen of Atractylodes chinensis in China. Mycological Progress 17, 393-402.

Xu S, Li YZ. 2015 - First report of common vetch anthracnose caused by Colletotrichum lentis in China. Plant Disease 99, 1859.

Xu SJ, Aktaruzzaman M, Kim BS, Kim JY et al. 2018b - First report of anthracnose caused by Colletotrichum fioriniae on eggplant fruits in Korea. Plant Disease 102, 2642.

Yamagishi N. 2017 - Studies on disease cycle and control of celery stunt anthracnose caused by Colletotrichum nymphaeae. Journal of General Plant Pathology 83, 429-430.

Yan JY, Jayawardena MMRS, Goonasekara ID, Wang Y et al. 2015 - Diverse species of Colletotrichum associated with grapevine anthracnose in China. Fungal Diversity 71, 233246.

Yang B, Jin X, Feng Q, Xiao K et al. 2020 - Colletotrichum species causing leaf spot diseases of Liriope cymbidiomorpha (ined.) in China. Australasian Plant pathology 49, 137-139.

Yang HC, Haudenshield JS, Hartman GL. 2012b - First report of Colletotrichum chlorophyti causing soybean anthracnose. Plant Disease 96, 1699.

Yang HC, Haudenshield JS, Hartman GL. 2014 - Colletotrichum incanum sp. nov., a curvedconidial species causing soybean anthracnose in USA. Mycologia 106, 32-42.

Yang L, Lu XH, Jing YL, Li SD et al. 2019a - First report of common bean (Phaseolus vulgaris) stem rot caused by Colletotrichum spaethianum in China. Plant Disease 103, 151-152.

Yang S, Wang HX, Yi YJ, Tan LL. 2019b - First report that Colletotrichum aenigma causes leaf spots on Camellia japonica in China. Plant Disease 103, 2127.

Yang Y, Cai L, Liu Z, Hyde KD et al. 2011 - Colletotrichum species on Orchidaceae in southwest China. Cryptogamie Mycology 32, 229-253.

Yang Y, Liu Z, Cai L, Hyde KD. 2012a - New species and notes of Colletotrichum on daylilies (Hemerocallis spp.). Tropical Plant Pathologyogy 37, 165-174.

Yang YL, Liu ZY, Cai L, Hyde KD et al. 2009 - Colletotrichum anthracnose of Amaryllidaceae. Fungal Diversity 39, 123-46.

Yang ZH, Rannala B. 2010 - Bayesian species delimitation using multilocus sequence data. Proceeding of the National Academy of Sciences USA. 107, 9264-9.

Young A, Gillung JP. 2020 - Phylogenomics-principles, opportunities and pitfalls of big-data phylogenetics. Systematic Entomology 45, 225-247.

Yuan HS, Lu X, Dai YC, Hyde KD et al. 2020 - Fungal Diversity Notes 1277-1386: taxonomic and phylogenetic contributions to fungal taxa. Fungal Diversity 104, 1-266.

Yu J, Wu J, Guo Z, Zhang X et al. 2020 - First report of peanut anthracnose caused by Colletotrichum truncatum in China. Plant Disease 104, 1555.

Zampounis A, Pigné S, Dallery JF, Wittenberg AH et al. 2016 - Genome sequence and annotation of Colletotrichum higginsianum, a causal agent of crucifer anthracnose disease. Genome announcements 4, article 4.

Zeilinger S, Gupta VK, Dahms TE, Silva RN et al. 2016 - Friends or foes? Emerging insights from fungal interactions with plants. FEMS microbiology reviews 40, 182-207.

Zhai YX, Zhang BQ, Cao H, Hao XJ. 2018 - First report of Colletotrichum truncatum causing anthracnose of begonia in China. Plant Disease 102, 1177-1178. 
Zhang FF, Wang MZ, Zheng YX, Liu HY et al. 2015 - Isolation and characterzation of endophytic Huperzine - A producing fungi from Phlegmariurus phlegmaria. Microbiology 84, 701-709.

Zhang Y, Xu H, Jiang S, Wang F et al. 2018 - First report of Colletotrichum truncatum causing anthracnose on the berry stalk and the rachis of kyoho grape (Vitis labruscana $\mathrm{x}$ V. vinifera) clusters in Hebei, China. Plant Disease 102, 2040.

Zhang YB, Meng K, Shu JP, ZhangW, Wang HJ. 2019 - First report of anthracnose on Pecan (Carya illinoensis) caused by Colletotrichum nymphaeae in China. Plant Disease 103, 1432.

Zhang W, Damm U, Crous PW, Groenewald JZ et al. 2020 - Anthracnose disease of carpet grass (Axonopus compressus) caused by Colletotrichum hainanense sp. nov. Plant Disease 104, 1744-1750.

Zhao M, Qiu H, Jiang H, Zhang Z et al. 2012 - Optimization of fermentation conditions of biocontrol strain Col-68 Colletotrichum hanaui against Digitaria sanguialis. Acta Agriculturae Zhejiangensis 24, 459-463.

Zhao W, Wang T, Chen QQ, Chi YK et al. 2016a - First report of Colletotrichum scovillei causing anthracnose fruit rot on pepper in Anhui Province, China. Plant Disease 100, 2168.

Zhao W, Wang T, Chen QQ, Chi YK et al. 2016b - First report of leaf spot caused by Colletotrichum spaethianum on Lilium lancifolium in China. Plant Disease 100, 2328.

Zhong J, Li CX, Zhong SY, Hu Z. 2020 - First report of leaf spot caused by Colletotrichum spaethianum on Paris polyphylla in China. Plant Disease 104, 972.

Zhou S, Qiao L, Jayawardena RS, Hyde KD et al. 2019 - Two new endophytic Colletotrichum species from Nothapodytes pittosporoides in China. MycoKeys 49, 1-14.

Zhou Y, Huang JS, Yang LY, Wang GF et al. 2017 - First report of banana anthracnose caused by Colletotrichum scovillei in China. Plant Disease 101, 381-382.

Zimmermann A. 1901 - Die thierischen und pflanzlichen Feinde der Kautschuk-und GuttaperchaPflanzen. Bulletin de I'lnstitut Botanique de Buitenzorg. 10, 1-27.

Zimmermann A. 1904 - UntersuchungenübertropischePflanzenkrankheiten. Mitteilung I. Berichtüber Land-und Forstwirtschaft in Deutsch-Ostafrika. 2, 11-36. 